\title{
An Ecological Land Survey for Fort Richardson, Alaska
}

M. Torre Jorgenson, Joanna E. Roth, Sharon F. Schlentner, Erik R. Pullman, Matt Macander, and Charles H. Racine

September 2003

US Army Corps of Engineers ${ }_{\circledast}$

Engineer Research and Development Center 
Abstract: An ecological land survey (ELS) of Fort Richardson land was conducted to map ecosystems at three spatial scales to aid in the management of natural resources. In an ELS, an attempt is made to view landscapes not just as aggregations of separate biological and earth resources, but as ecological systems with functionally related parts that can provide a consistent conceptual framework for ecological applications. Field surveys at 132 plots along 16 toposequences and at 99 other plots were used to identify relationships among physiography, geomorphology, soils, hydrology, and vegetation. The relationships revealed that the various ecosystem components were closely related to fire effects and geomorphic processes, such as floodplain development, landslide and slope instability, and coastal flooding. Associations among vegetation structures and geomorphic units were used to identify 51 ecotypes (local-scale ecosystems) that were effective at differentiating dominant species and plant associations. Ecosystem maps were developed at three spatial scales. Forty-six ecotypes (1:20,000 scale), derived from the integrated terrain unit (ITU) mapping, differentiated areas with homogeneous topography, terrain, soil, surface form, hydrology, and vegetation. Vegetation (structure and composition) and environmental (elevation, organic matter accumulation, depth to rock, water depths, $\mathrm{pH}$, and electrical conductivity) characteristics of ecotypes were summarized using data obtained from field surveys. Sixteen ecosections $(1: 100,000$ scale) were aggregated from the ecotypes to differentiate areas that are homogeneous with respect to geomorphic features and soil texture, and thus have recurring patterns of soils and vegetation at various successional stages. Four ecodistricts and eight ecosubdistricts $(1: 250,000)$ were developed from separate mapping of Landsat imagery to differentiate broader areas with similar physiography, geology, and geomorphology. This hierarchical linkage of ecological characteristics within a spatial database facilitates the evaluation of land capabilities and sensitivities and provides flexibility for addressing a wide range of land management objectives.

COVER: View from upper Snowhawk Creek Valley illustrating three ecodistricts across Fort Richardson, including the Northern Chugach Mountains (foreground), the Matanuska-Susitna Lowlands (background) and Cook Inlet Coast (background).

\section{How to get copies of ERDC technical publications:}

Department of Defense personnel and contractors may order reports through the Defense Technical Information Center:

DTIC-BR SUITE 0944

8725 JOHN J KINGMAN RD

FT BELVOIR VA 22060-6218

Telephone (800) 225-3842

E-mail help@dtic.mil msorders@dtic.mil

WWW http://www.dtic.mil/

All others may order reports through the National Technical Information Service:

NTIS

5285 PORT ROYAL RD

SPRINGFIELD VA 22161

Telephone (703) 487-4650

(703) 487-4639 (TDD for the hearing-impaired)

E-mail orders@ntis.fedworld.gov

WWW http://www.ntis.gov/index.html

For information on all aspects of the Engineer Research and Development Center, visit our World

Wide Web site:

http://www.erdc.usace.army.mil 


\section{An Ecological Land Survey for Fort Richardson, Alaska}

M. Torre Jorgenson, Joanna E. Roth, Sharon F. Schlentner, Erik R. Pullman, Matt Macander, and Charles H. Racine

Cold Regions Research and Engineering Laboratory

72 Lyme Road

Hanover, New Hampshire 03755

Approved for public release; distribution is unlimited.

Prepared for U.S. ARMY ALASKA, DIRECTORATE OF PUBLIC WORKS 600 Richardson Avenue, Fort Richardson

Anchorage, Alaska 99505 


\section{PREFACE}

This report was prepared by M. Torre Jorgenson, Senior Scientist, Joanna E. Roth, Research Biologist, Sharon F. Schlentner, Research Biologist, Erik R. Pullman, Senior Research Biologist, and Matt Macander, GIS Specialist, ABR, Inc., Fairbanks, Alaska.

Funding for this work was provided by Fort Richardson, Alaska.

The authors acknowledge the support of Gary Larsen, Terry Schick, Bill Gossweiler, and Bill Quirk at Fort Richardson. Dr. Charles Racine, U.S. Army Engineer Research and Development Center, Cold Regions Research and Engineering Laboratory, Hanover, New Hampshire, provided data for coastal ecosystems on the Eagle River Flats. Todd Sforma and Jason Ontjes assisted in the fieldwork. Stephen Murphy and Susan Bishop technically reviewed this report. Jennifer Felkay helped to produce the draft report.

The Commander and Executive Director of the Engineer Research and Development Center is Colonel James R. Rowan, EN. The Director is Dr. James R. Houston. 


\section{CONTENTS}

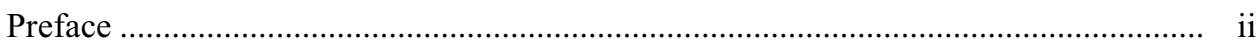

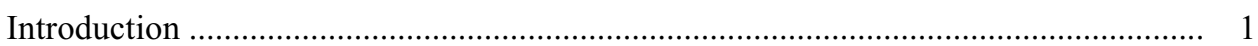

Ecological land survey approach .................................................................. 1

Fort Richardson ecological land survey ........................................................... 3

History of Fort Richardson development ………............................................. 3

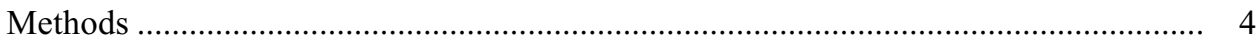

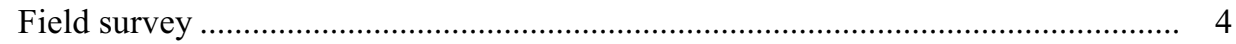

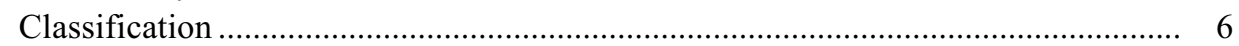

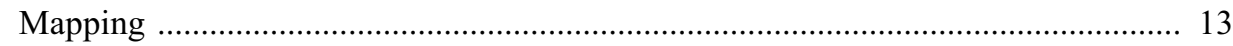

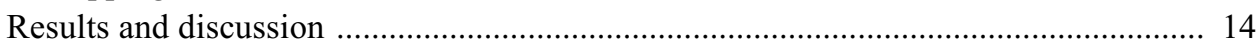

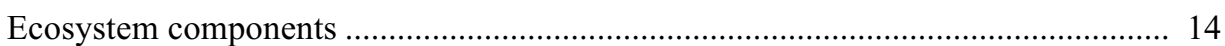

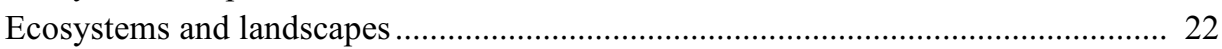

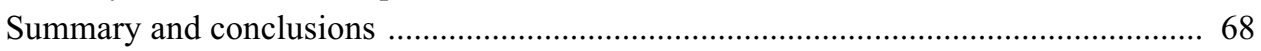

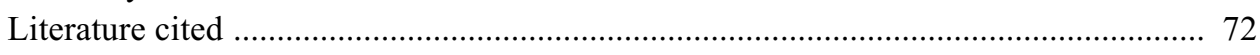

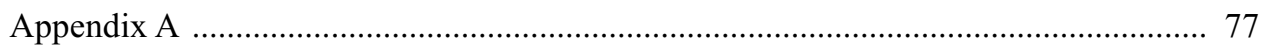

Appendix B …………… 83

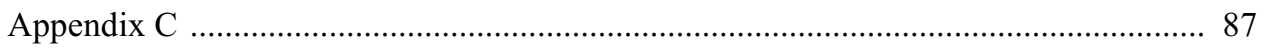

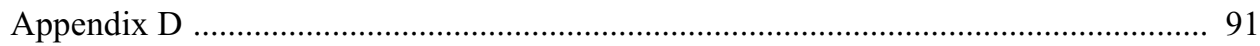

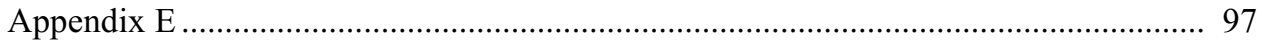

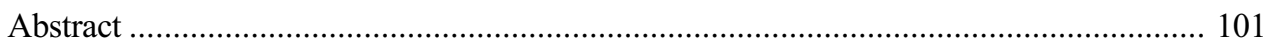

\section{ILLUSTRATIONS}

Figure

1. Interaction of interrelated state factors that control structure and function

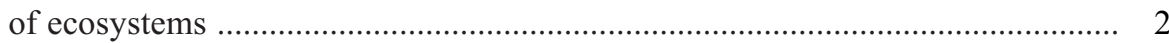

2. Location of study area and sampling locations for the ecological land classification on Fort Richardson, south-central Alaska, 2001 ......................... 5

3. System of hierarchically classifying ecosystem components into integrated

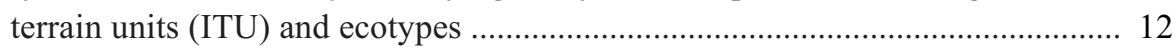

4. Climate diagram (mean monthly temperature and precipitation) for the Anchorage station near Fort Richardson, south-central Alaska .. 14

5. Map of the geology of the region surrounding Fort Richardson, south-central Alaska, 2001

6. Map of geomorphic units on Fort Richardson, south-central Alaska, 2001

7. Map of topography illustrated as shaded relief for Fort Richardson, southcentral Alaska, 2001

8. Map of surface forms on Fort Richardson, south-central Alaska, 2001 .............. 20

9. Map of vegetation classes on Fort Richardson, south-central Alaska, 2001

10. Toposequence on the Eagle River Flats along the Upper Cook Inlet Coast illustrating geomorphology, vegetation, elevations, and soil stratigraphy, Fort Richardson, south-central Alaska, 2001 
11. Toposequence (Transect 1) on the Eagle River Floodplain illustrating geomorphology, vegetation, elevations, and soil stratigraphy, Fort

Richardson, south-central Alaska, 2001

12. Toposequence (Transect 2) near Camp Carrol in the Anchorage Glaciated Lowlands illustrating geomorphology, vegetation, elevations, and soil stratigraphy, Fort Richardson, south-central Alaska, 2001 30

13. Toposequence (Transect 9) near Chain Lake in the Knik Glaciated Lowlands illustrating geomorphology, vegetation, elevations, and soil stratigraphy, Fort Richardson, south-central Alaska, 2001 30

14. Toposequence (Transect 15) on the Anchorage Glaciated Hillsides illustrating geomorphology, vegetation, elevations, and soil stratigraphy, Fort Richardson, south-central Alaska, 2001

15. Toposequence (Transects 11 and 12) in Snowhawk Valley in the Northern Chugach Mountains illustrating geomorphology, vegetation, elevations, and soil stratigraphy, Fort Richardson, south-central Alaska, 2001

16. Ground views of alpine, subalpine, upland forest, and lowland forest ecotypes on Fort Richardson, south-central Alaska, 2001

17. Ground views of lowland, lacustrine, riverine, and coastal ecotypes on Fort Richardson, south-central Alaska, 2001

18. Map of ecotypes on Fort Richardson, south-central Alaska, 2001

19. Percent cover of growth forms by ecotype on Fort Richardson, south-central Alaska, 2001

20. Detrended correspondence analysis of alpine and subalpine plots and associated plant species analyzed from Fort Richardson, south-central Alaska, 2001

21. Detrended correspondence analysis of lowland, riverine, and upland plots and associated plant species analyzed from Fort Richardson, south-central Alaska, 2001

22. Detrended correspondence analysis of coastal plots and associated plant species analyzed from Fort Richardson, south-central Alaska

23. Mean $( \pm \mathrm{SD})$ elevation, organic matter thickness, and depth to gravel of ecotypes on Fort Richardson, south-central Alaska, 2001

24. Mean $( \pm \mathrm{SD}) \mathrm{pH}$, electrical conductivity (EC), and water depth (negative when below ground) for ecotypes on Fort Richardson, south-central Alaska, 2001

25. Mean $( \pm \mathrm{SD})$ elevation, organic matter thickness, and depth to gravel for abundant plant species on Fort Richardson, south-central Alaska, 2001

26. Mean $( \pm \mathrm{SD}) \mathrm{pH}$, electrical conductivity (EC), and water depth (negative when below ground) for abundant plant species on Fort Richardson, southcentral Alaska, 2001

27. Map of human disturbances on Fort Richardson, south-central Alaska, 2001 64

28. Map of ecosections based on geomorphic units for Fort Richardson, south-central Alaska, 2001

29. Map of ecodistricts and ecosubdistricts in the vicinity of Fort Richardson, south-central Alaska, 2001 


\section{TABLES}

Table

1. Coding system for classifying and mapping geomorphic units, surface forms, vegetation, and disturbance on Fort Richardson, south-central Alaska, 2001 .... 7

2. Comparison of systems for differentiating ecosystems at various scales ............. 8

3. Classification and description of geomorphic units within Fort Richardson, south-central Alaska, 2001 ..................................................................... 9

4. Areal extent of geomorphic units and surface forms mapped on Fort Richardson, south-central Alaska, 2001

5. Classification and descriptions of surface forms within Fort Richardson, south-central Alaska, 2001

6. Classification and description of vegetation within Fort Richardson, southcentral Alaska, 2001

7. Areal extent of vegetation classes mapped on Fort Richardson, south-central Alaska, 2001

8. Relationships among ecological components of ecosystems found within Fort Richardson, south-central Alaska, 2001

9. Classification and description of ecotypes found within Fort Richardson, south-central Alaska, 2001

10. Areal extent of ecotypes mapped on Fort Richardson, south-central Alaska, 2001

11. Mean cover $(\%)$ of the most abundant species within alpine and subalpine ecotypes on Fort Richardson, south-central Alaska, 2001

12. Mean cover $(\%)$ of the most abundant species within upland and lowland forest ecotypes on Fort Richardson, south-central Alaska, 2001

13. Mean cover $(\%)$ of the most abundant species within upland and lowland scrub, and lacustrine ecotypes on Fort Richardson, south-central Alaska, 2001

14. Mean cover (\%) of the most abundant species within riverine ecotypes on Fort Richardson, south-central Alaska, 2001

15. Mean cover (\%) of the most abundant species within coastal ecotypes on Fort Richardson, south-central Alaska, 2001

16. Classification, description, and areal extent of disturbances mapped on Fort Richardson, south-central Alaska, 2001

17. Classification and description of ecosections within Fort Richardson, southcentral Alaska, 2001

18. Areal extent of ecosections mapped on Fort Richardson, south-central Alaska, 2001

19. Classification and description of ecosubdistricts in the vicinity of Fort Richardson, south-central Alaska, 2001 


\title{
An Ecological Land Survey for Fort Richardson, Alaska
}

\author{
M. TORRE JORGENSON, JOANNA E. ROTH, SHARON F. SCHLENTNER, \\ ERIK R. PULLMAN, MATT MACANDER, AND CHARLES H. RACINE
}

\section{INTRODUCTION}

In response to the need for information on the natural resources of Fort Richardson, we performed an ecological land survey (ELS) within the boundaries of the base. This information is needed for ongoing resource management activities on the base, including the Integrated Training Area Management program implemented by the U.S. Army (USARAK 1998). This report presents the rationale and methods used to classify and map ecosystems on the base, describes the nature and dynamics of these ecosystems, and documents the structures of the GIS databases used in mapping and aggregating ecosystems at several spatial scales.

Spatial databases developed from an ecological land classification are essential to managing land resources and have many applications, such as ecological risk assessments, analysis of terrain sensitivity and wildlife habitats, wetland protection, mitigation planning for training exercises, facility location, identification of rare habitats, and fire management. By delineating areas with co-varying climate, geomorphology (surficial geology and terrain units), surface forms, hydrology, and vegetation, the resulting maps provide a spatial stratification that is particularly useful for integrated resource management based on GIS. This hierarchy of scales can help land managers and military trainers access information, identify information gaps, and improve resource management of large areas.

\section{Ecological land survey approach}

In an ELS, landscapes are viewed not just as aggregations of separate biological and earth resources, but as ecological systems with functionally related parts (Rowe 1961; Wiken and Ironside 1977; Bailey 1980, 1996; Driscoll et al. 1984). The goal of an ELS, then, is to provide a consistent conceptual framework for modeling, analyzing, interpreting, and applying ecological knowledge. To provide the information required for such a wide range of applications, an ELS involves three types of effort: (1) an ecological land survey that inventories and analyzes data obtained in the field, (2) an ecological land classification that classifies and maps ecosystem distribution, and (3) an ecological land evaluation that assesses the capabilities of the land for various land management practices. Our emphasis in this report is on the ecological land survey and classification efforts. A companion report evaluates some of the potential land evaluation applications, such as disturbance regimes, soil erosion potential, and wildlife habitat use (Jorgenson et al. 2002).

The structure and function of natural ecosystems are regulated largely along gradients of energy, moisture, nutrients, and disturbance. These gradients are affected by climate, physiography, geomorphology, soils, hydrology, vegetation, and fauna, which can be viewed as ecosystem components or "state factors" (Barnes et al. 1982, ECOMAP 1993, Bailey 1996). Accordingly, we used the state-factor approach (Jenny 1941, Van Cleve et al. 1990, Vitousek 1994, Bailey 1996, Ellert et al. 1997) both to partition the variation in local ecosystems using differences in independent factors (e.g., climate, geomorphology, topography, parent material, and time), and to facilitate ecosystem classification and mapping (Fig. 1a). While thematic maps of individual ecosystem components (e.g., geomorphology and vegetation) have their particular uses, this linking and aggregating of components into ecosystems with co-varying climate, geomorphology, surface forms, hydrology, and vegetation can provide a spatial stratification that conveys a much broader range of information required for ecosystem management. 
a)

b)
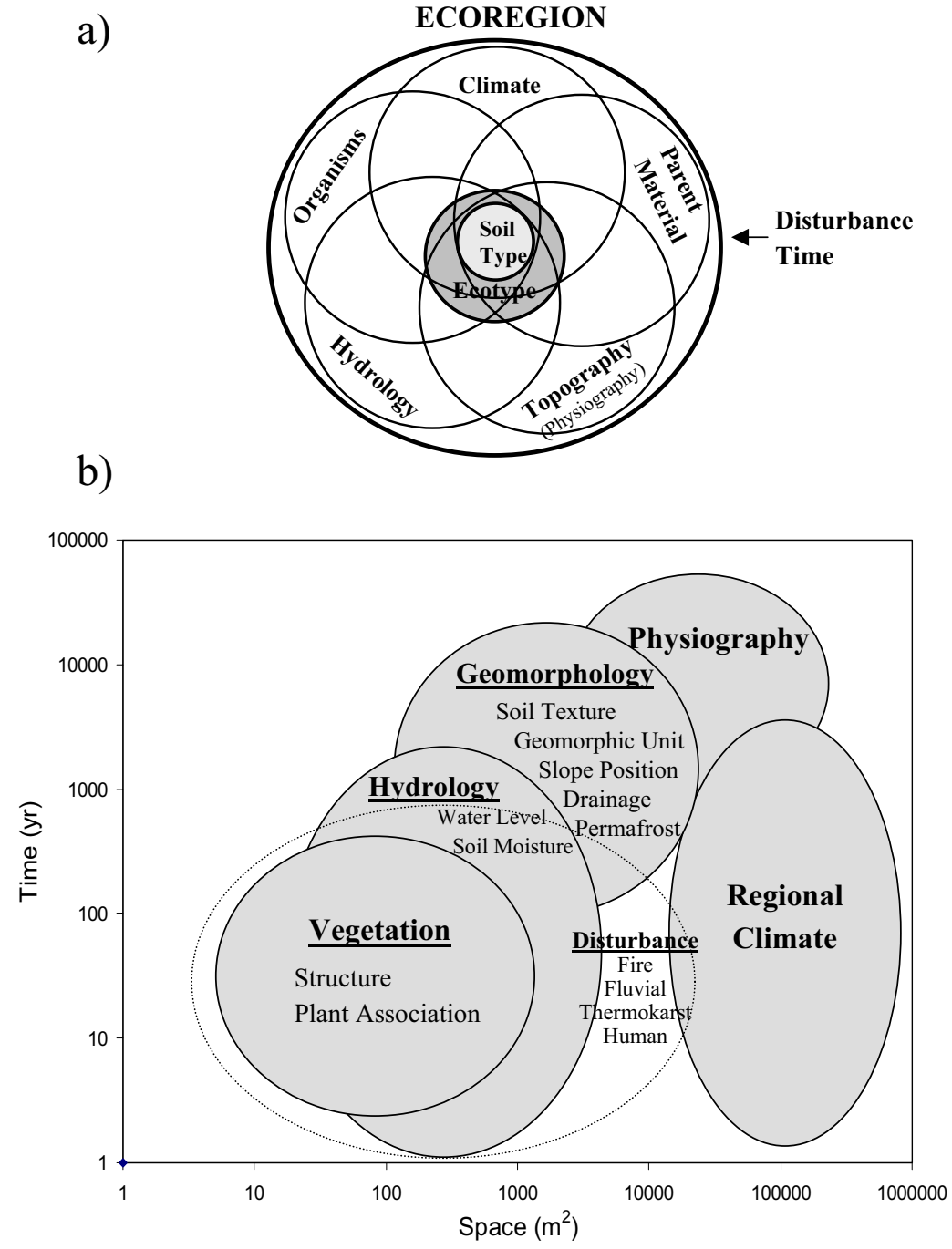

Figure 1. Interaction of interrelated state factors that control structure and function of ecosystems (a). Ecotypes are local-scale ecosystems comprised of various ecosystem components that exist within large regional ecosystems, or ecoregions. Factors affecting ecosystem distribution occur over a range of scales within a nested hierarchy (b).

An ecological land classification also involves the organization of ecosystem components at various scales (Wiken 1981, O’Neil et al. 1986, Klijn and Udo de Haes 1994,Bailey 1996) based on the recognition that the state factors operate within a hierarchy of differing spatial and temporal scales (Allen and Starr 1982, Delcourt and Delcourt 1988, Forman 1995). This hierarchical linkage reveals that local scale features, such as vegetation, are nested within regional scale components, such as climate or physiography (Fig. 1b). Climate, particularly temperature and precipitation, accounts for the largest amount of variation in ecosystem structure and function globally (Walter 1979, Vitousek 1994, Bailey 1998). Within a given climate, physiography (characteristic geologic substrate, surface shape, and relief) creates the elevation gradients that control the spatial arrangement and rate of geomorphic processes (characteristic lithologies or soil texture) and energy flows. Geomorphic units with characteristic lithologies, texture, and surface forms affect soil properties and the movement of water in soil (Wahrhaftig 1965, Swanson et al. 1988, Bailey 1996). Patterns of water movement are critical factors in water balance and nutrient availability for plants and thus affect the distribution of veg- 
etation (Fitter and Hay 1987, Oberbauer et al. 1989). Vegetation technically is the most important factor controlling the trophic structure of ecosystems, because it provides structure and energy for other trophic levels, controls material and energy exchange, and affects soil erosion and geomorphic processes (Walter 1979, Bailey 1996). Finally, natural and human disturbances have long been recognized as important factors affecting the timing and development of ecosystems (Watt 1947, Pickett et al. 1989, Walker and Walker 1991, Forman 1995).

Beyond this conceptual framework of state-factor control, however, there is no single natural scale at which ecological phenomena should be studied. This leads observers to impose their own perceptual bias in the study of patterns and processes of ecological phenomena (Levin 1992, Shugart 1998). In addition, there is no nationally accepted approach to classifying ecosystems, although recent efforts have been made to develop a consensus among federal agencies (ECOMAP 1993) and among nations (Klijn and Udo de Haes 1994, Uhling and Jordan 1996). In this report, we generally have followed the scales and differentiating criteria described by Klijn and Udo de Haes (1994), which combine elements of both the Canadian (Wiken and Ironside 1977) and U.S. systems (ECOMAP 1993). This combined system involves numerous spatial scales for mapping ecosystems and identifies various ecosystem components as the prime criteria for differentiating successive levels of hierarchical organization.

In Alaska, a hierarchical approach to mapping vegetation and land cover has been developed for northern Alaska by Walker and his colleagues (Walker 1983, 1999). They also applied an integrated, geobotanical approach to mapping ecosystem components in the Prudhoe Bay region, but they did not group the integrated units hierarchically (Walker et al. 1980). Recently, an integrated-terrain-unit approach has been used for large-scale mapping of ecosystems on the Arctic Coastal Plain (Jorgenson et al. 1997), in interior Alaska at Fort Wainwright (Jorgenson et al. 1999) and Fort Greely (Jorgenson et al. 2001), and for vegetation complexes across the entire North Slope (Walker 1999). When applied to coastal ecosystems on the YukonKuskokwim Delta, the hierarchical organization of ecosystems at several spatial scales was effective at partitioning the variability of numerous biotic and abiotic variables (Jorgenson 2000).

\section{Fort Richardson ecological land survey}

In this report, we present and evaluate three levels of ecosystem organization; ecotypes (1:20,000 scale), ecosections $(1: 100,000)$, and ecodistricts $(1: 500,000)$. Ecotypes (also referred to as local ecosystems, ecotopes, or landtype phases) delineate areas with homogenous geomorphology (lithofacies related to depositional or erosional process), surface form (topographic shape), soil, hydrology, and vegetation. Ecosections (also landscapes, landtype associations, or geomorphic sections) are homogeneous with respect to geomorphic features and have recurring patterns of water regime, soil, and vegetation. Several vegetation classes can be included in an ecosection, but they are usually stages in a single successional sequence. Ecodistricts (also subregions or physiographic districts) are broader areas with similar geology, geomorphology, and hydrology. In addition to the three levels that we mapped for Fort Richardson, the broader level of ecoregions (or climatic zones), which differentiate areas based on their climatic regimes and gross physiography, have been mapped recently for Alaska by Gallant et al. (1995) and Nowacki et al. (2002).

In conducting this ecological land survey, we benefited from numerous earlier studies of various ecological components of Fort Richardson. Information on surficial geology was compiled and updated by Hunter et al. (2000), based on earlier mapping by Schmoll and Dobrovolny (1972), Reger and Updike (1983), Yehle and Schmoll (1987, 1989), Yehle et al. (1990), and Schmoll et al. (1996). The soils of the base were inventoried and mapped by the Natural Resource Conservation Service (NRCS 2000). Vegetation surveys have been conducted for the Eagle River Flats (Racine et al. 1993, unpublished data), alpine areas (Walker et al. 1997), and for old growth forests (Marler and Vankat 1997), and an unpublished vegetation map was produced by Colorado State University in 1998. Lichvar et al. (1997) conducted a floristic inventory of the base.

Wherever possible, we incorporated classifications and boundary delineations of previous studies into our ecological mapping. However, maps from the various studies used different base maps, and linework usually did not match up with the newly available, high-quality digital orthophoto mosaic recently produced for the base. Consequently, we developed an entirely new map controlled to the orthophoto mosaic. While we referred to the existing surficial geology and soils maps as much as possible, we needed to make numerous compromises to integrate the various ecological components into a unified map. Integration of the ecological components improves upon earlier independent thematic mapping by providing one layer of linework that includes multiple-parameter coding for geomorphology, surface form, and vegetation.

\section{History of Fort Richardson development}

The nature and distribution of ecosystems on Fort Richardson have also been greatly affected by human 
activities. Thus, the history of human use and management of the land becomes a part of the ecological history of the landscape and much of this human history is driven by political and economic forces far removed from Alaska (Johnson and Jorgenson 1963). The outbreak of World War II in Europe in 1939, subsequent invasion of the Aleutians by the Japanese in 1942, the expansion of defense construction during the Cold War, and development of rapid deployment forces during the 1980s and 1990s have all contributed to modification of the landscape at Fort Richardson. The following history is summarized from USARAK (2001).

Fort Richardson and its air establishment, Elmendorf Field, were established in 1939 by an Executive Order (EO) that withdrew 14,800 ha $(36,570 \mathrm{ac})$ of land from the public domain, placing it under jurisdiction of the War Department. Between 1939 and 1945, 61,180 ha $(151,180 \mathrm{ac})$ were withdrawn for military use. Fort Richardson originally resided on land that Elmendorf Air Force Base (AFB) currently occupies. In 1950, Fort Richardson was moved east to its current location, and 36,60 ha $(9,042 \mathrm{ac})$, which later became Elmendorf AFB, were transferred to the Air Force. From 1945 to 1955 , the military returned to the Department of the Interior $\sim 34,400$ ha $(85,000 \mathrm{ac})$ that were not needed for military use. From 1955 to 1965 , the Department of the Army released $\sim 4050$ ha $(10,000 \mathrm{ac})$ to various entities, such as the U.S. Air Force, State of Alaska, and the Bureau of Land Management (BLM), and acquired $\sim 2430$ ha $(6,000 \mathrm{ac})$ for Army use. From 1966 to the present, Fort Richardson's boundaries have remained fairly stable. Leases from the BLM have expanded the eastern and southern boundaries.

Most of Fort Richardson's infrastructure initially was constructed during the post-war era from 1947 to 1960. Army troops were redesignated as the United States Army Alaska (USARAL) in 1947 and headquarters was established at Fort Richardson. The early 1950 s saw an intensive building program and by 1960 most of the Fort's major facilities were completed. Three off-post Nike-Hercules missile sites were built in 1959 and the missile unit remained active until July 1979.

In 1974, as part of worldwide realignments, USARAL was inactivated and the post became headquarters for the 172nd Infantry Brigade (Alaska) in 1975. In a subsequent realignment in 1986, the 172nd gave way to the 6th Infantry Division (Light) and United States Army Garrison, Alaska. This marked a new mission for the Army in Alaska as a light force capable of being deployed worldwide. The division became aligned more closely with the Defense Department's forces in the Pacific when, in 1989, it began reporting to the U.S. Army Western Command in Hawaii (later redesignated United States Army Pacific). In 1990, headquarters for the 6th was moved to Fort Wainwright and in 1993, as part of Army-wide downsizing, the 6th was reorganized as a light infantry brigade. The 6th Infantry Division (Light) was inactivated 1994, and Fort Richardson became headquarters for United States Army Alaska (USARAK). In 1998, the 1st Brigade, 6th Infantry Division (Light) was deactivated, and the 172nd Infantry Brigade was reactivated. Training associated with the 172nd Infantry Brigade (Alaska) and the 6th Infantry Division (Light) resulted in the development and expansion of training facilities on the northern half of the base.

Natural resource management activities have also contributed to ecological changes on the base. Past and proposed activities are discussed in detail in the Integrated Natural Resources Management Plan 1998-2003 for Fort Richardson (USARAK 1998); here we provide a brief summary. In the mid-1950s, fish and wildlife management personnel stocked rainbow trout in some post lakes (Gossweiler 1984 as cited in USARAK 1998). In the early 1960s, the cooling pond at the power plant was used to raise fish collected from a local hatchery, and stocking efforts expanded to include steelhead trout, kamloop trout, silver salmon, and king salmon. In 1956 and 1957, wild rice was sown on Eagle River Flats to improve waterfowl habitat. In 1975, intensive management of forests to enhance moose habitat was initiated and included clear-cutting, or clearing with a Hydro-Ax, older forests to stimulate moose-browse production (Quirk et al. 1978 as cited in USARAK 1998, Gossweiler 1984). During 1975-1980, 100 ha $(250 \mathrm{ac})$ were cleared to enhance moose habitat and the program has been expanded to include over 600 ha $(1500 \mathrm{ac})$. The active natural resource management program on Fort Richardson has been awarded the Citation of Meritorious Achievement by the Secretary of Defense in 1969 and 1971, and the Environmental Award for Natural Resources Conservation by the Secretary of the Army in 2000.

\section{METHODS}

\section{Field survey}

Field sampling was conducted on Fort Richardson ( 25,000 ha, 62,000 acres) in July and August 2000. A gradient-directed sampling scheme (Austin and Heyligers 1989) was followed to sample the range of ecological conditions and provide the spatial relationships necessary to interpret ecosystem development. Sixteen transects $(\sim 1 \mathrm{~km})$ were selected describing a range of physiographic classes from lowland to alpine. Along each transect or toposequence, 8-12 plots were sampled, each in a distinct vegetation type or spectral 
signature identifiable on aerial photographs. Data were collected at 132 plots along toposequences and an additional 99 plots were sampled off transects to increase replication of under-represented or rare classes (Fig. 2). All plots were located on aerial photographs, and coordinates (including approximate elevations) were obtained with a Global Positioning System (GPS) receiver. At each sample plot ( $\sim 10$-m radius) descriptions of geology, surface form, hydrology, near-surface soil stratigraphy, permafrost occurrence, and vegetation were recorded. Digital ground photos were taken at all plots; data and photos are archived at ABR, Inc. Access to the tidal flats at Eagle River Flats was not pos- sible because of unexploded ordnance, so data collected previously (Racine et al. 1993, unpublished data) were used for analysis of coastal ecotypes and vegetation types.

Geologic and surface-form variables that were recorded included physiography, surface geomorphic unit, slope, aspect, surface-form class, and depth of microrelief.

Hydrologic data included measurements of depths to water and saturated soil, $\mathrm{pH}$, electrical conductivity (EC), and temperature. Water quality measurements were made with Oakton or Cole-Palmer pocket meters that were calibrated at regular intervals in the field to

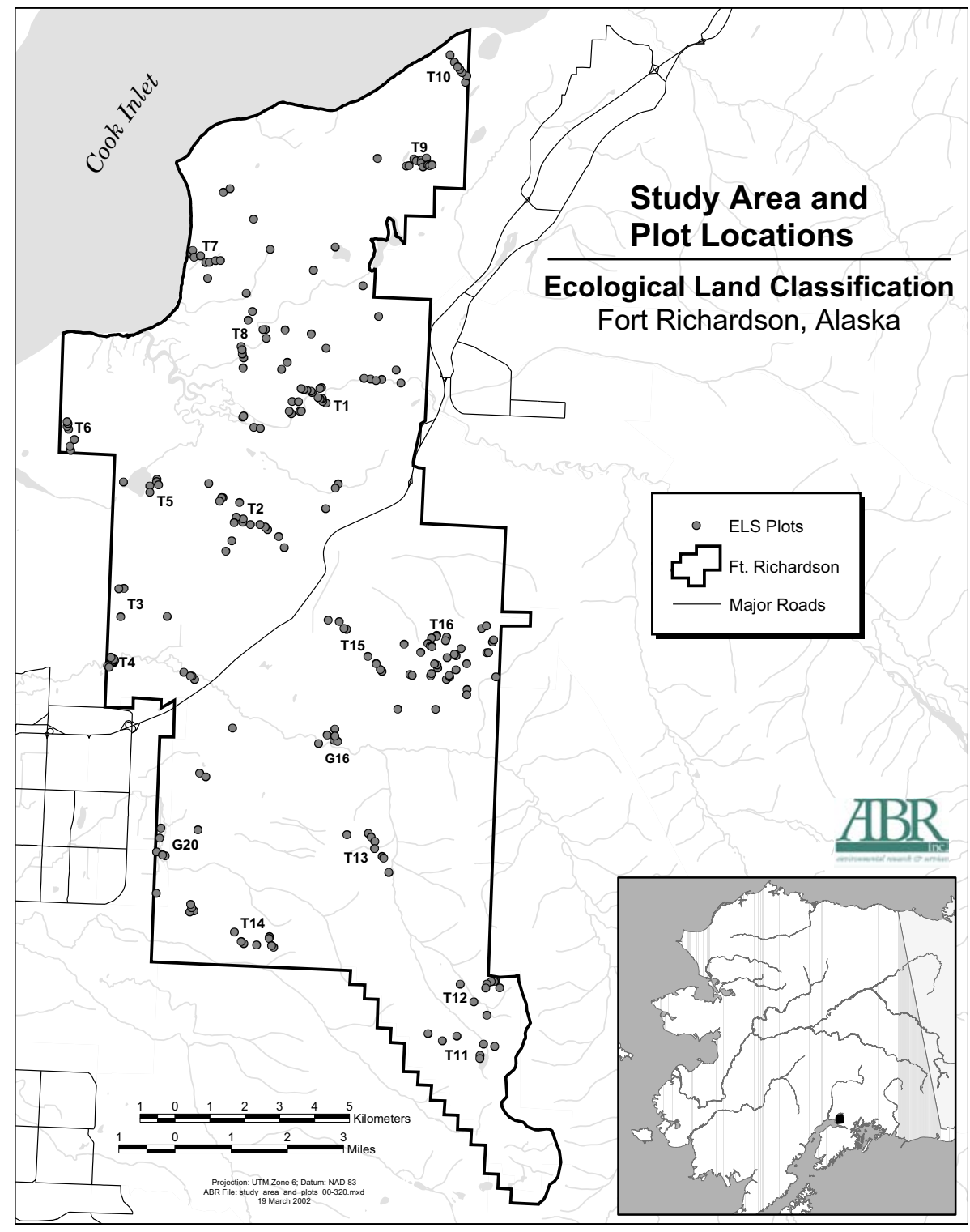

Figure 2. Location of study area and sampling locations for the ecological land classification on Fort Richardson, south-central Alaska, 2001. 
standards appropriate for within the range of use. When water was not present in the soil pit, $\mathrm{pH}$ and $\mathrm{EC}$ were determined in the field from a saturated paste of distilled water and soil collected at 10-20 cm depth.

Soil stratigraphy was described from soil plugs dug with a shovel to approximately $50 \mathrm{~cm}$ using standard methods (SSDS 1993). Where possible, a soil corer or tile probe was used to extend the sampling depth description and to determine the depth to underlying gravel or permafrost, if present. Descriptions of each profile included texture and color of each horizon, depth of organic layer, type and percentage of coarse fragments, depth to rock ( $>15 \%$ by volume), and the presence and character of mottling. Similar horizons, or repeating groups of textures, were grouped into lithofacies for depiction on toposequences, and a single simplified texture (i.e., rocky, sandy, loamy, clayey, or organic) was assigned to characterize the dominant texture at the plot for ecotype classification.

Vegetation structure and composition were assessed semi-quantitatively. Percent cover of each species was visually estimated to the nearest $5 \%$ if $>10 \%$ and to the nearest $1 \%$ if $<10 \%$. A species list was compiled for each plot. Total cover of each growth-form class (e.g., trees, tall shrubs, low shrubs, graminoids) was evaluated independently of cover of individual species and cross-checked to ensure the cover of the individual species with similar growth form added up to the cover estimated for the total growth form. Most species were identified in the field using Hultén (1968), and taxonomic nomenclature followed a previous floristic inventory referenced to the University of Alaska Museum collection (Lichvar et al. 1997). Unknown species were collected for later identification. Populus balsamifera balsamifera and P. balsamifera trichocarpa readily hybridize in the study area. Because differentiation of the two is uncertain, we assigned specimens from the subalpine zone to P. balsamifera balsamifera and referred to all other P. balsamifera as P. balsamifera trichocarpa, recognizing that hybrids between the two exist. Mosses, hepatics, and lichens that could not be identified in the field were sent to Olga Sumina, St. Petersburg University, and Mikhail Zurbenko, Komarov Botanical Institute, for identification. Primary references used by Drs. Sumina and Zurbenko were Thomson (1984) and Vitikainen (1994) for lichens, and Ignatov and Afonina (1992) and Konsantinova et al. (1992) for mosses and hepatics, respectively. Nomenclature used by Sumina and Zurbenko was revised to match that used in the floristic inventory (Lichvar et al. 1997) when possible, or to the National Plants Database (NRCS 2001) for those few cryptograms that were not previously identified.

\section{Classification}

Ecosystem classification was approached at two levels. First, individual ecosystem components were classified and coded using standard classification systems developed for Alaska (Table 1). Second, these ecosystem components were integrated to classify ecosystems at three spatial scales using a variety of differentiating criteria (Table 2).

\section{Ecological components}

Geomorphic units were classified according to a system based on landform-soil characteristics (Table 3). The system was originally developed for Alaska by Kreig and Reger (1982) and the Alaska Division of Geological and Geophysical Surveys (1983), and modified for this study. For classification of geomorphic units, we also relied on the surficial geology map of Fort Richardson, which was compiled and revised by Hunter et al. (2000) based on the geologic mapping of Yehle and Schmoll (1987, 1989), Yehle et al. (1990, 1991), and Schmoll et al. (1996). Organic units were based on the wetland classification for Canada (NWWG 1988). In classifying and mapping geomorphic units we emphasized materials near the surface $(<1 \mathrm{~m})$, because they have more influence on ecological processes than do materials deeper in the profile. For example, we classified as colluvium some mountain deposits that were previously mapped as bedrock, and we differentiated several floodplain classes (active, inactive, and abandoned floodplains) in areas that had been mapped as alluvium (Hunter et al. 2000).

Surface forms (macrotopography) were classified according to a system modified from that of Schoeneberger et al. (1998). Microtopography was classified according to the periglacial system of Washburn (1973). Soils were classified according to Keys to Soil Taxonomy, Eighth Edition (Soil Survey Staff 1998).

Vegetation types were initially classified to Level IV of the Alaska Vegetation Classification (Viereck et al. 1992) based on structural and floristic criteria. After fieldwork was completed and unknown specimens were identified, floristic associations were assigned using a two-step process. TWINSPAN analysis (mjm Software, Gleneden Beach, Oregon) was used to differentiate plant associations. Relevant groups were selected and dominant and differential species identified. A dominant species is present at moderate-to-high cover values, while a differential species is one uniquely associated with that particular association. Each plant association name consists of the names of a dominant species and a differential species that characterize the association. Both vegetation classes and plant associations were used in ecosystem analysis. 
Table 1. Coding system for classifying and mapping geomorphic units, surface forms, vegetation, and disturbance on Fort Richardson, south-central Alaska, 2001.

\begin{tabular}{|c|c|c|c|}
\hline$\overline{\text { Code }}$ & Class & Code & Class \\
\hline & GEOMORPHIC UNITS & & SURFACE FORM \\
\hline $\mathrm{N}$ & Metamorphic Bedrock & $\mathrm{C}$ & Crest \\
\hline Cgy & Younger Rock Glacier & $\mathrm{Sb}$ & Bluff \\
\hline $\mathrm{Ctb}$ & Talus Bluff & St & Streambanks \\
\hline $\mathrm{Ctc}$ & Talus Cone & Sun & Upper Slope, north facing \\
\hline $\mathrm{Cl}$ & Landslide Deposits & Sus & Upper Slope, south facing \\
\hline $\mathrm{Ctm}$ & Hillside Colluvium & Sue & Upper Slope, east or west facing \\
\hline Gmy & Younger Moraine & Suc & Upper Slope, concave \\
\hline Gmo & Older Moraine & S1 & Lower Slope \\
\hline GFk & Kame Deposits & $\operatorname{Sln}$ & Lower Slope, north facing \\
\hline MG & Glaciomarine Deposits & Sls & Lower Slope, south facing \\
\hline GFo & Glaciofluvial Outwash & Sle & Lower Slope, east or west facing \\
\hline GFc & Glaciofluvial Channel & Slc & Lower Slope, concave \\
\hline GL & Glaciolacustrine Deposits & Slv & Lower Slope, convex \\
\hline $\mathrm{Ob}$ & Bogs & $\mathrm{Xm}$ & Moraine Complex, undulating \\
\hline Fhm & Headwater Moderately Steep Floodplain & $\mathrm{F}$ & Flat or Fluvial Related \\
\hline Fhl & Headwater Lowland Floodplain & $\mathrm{Fc}$ & Channel, Swale or Gut \\
\hline $\mathrm{Ff}$ & Alluvial Fan & $\mathrm{Fi}$ & Interfluv or Flat Bank \\
\hline Ffo & Old Alluvial Fan & Fbp & Point Bar \\
\hline $\mathrm{Ft}$ & Alluvial Terrace & $\mathrm{F} 1$ & Levee \\
\hline FGp & Alluvial Plain Deposits & $\mathrm{Ff}$ & Flood Basin \\
\hline Fmra & Meander Active Channel Deposits & $\mathrm{B}$ & Basins or Depressions \\
\hline Fmri & Meander Inactive Channel Deposits & $\mathrm{Bt}$ & Thermokarst \\
\hline Fmrb & Meander Abandoned Channel Deposits & $\mathrm{U}$ & Undulating \\
\hline Fmoa & Meander Active Overbank Deposits & $\mathrm{W}$ & Waterbody \\
\hline Fmoi & Meander Inactive Overbank Deposits & $\mathrm{Hm}$ & Human Modified \\
\hline Fmob & Meander Abandoned Overbank Deposits & & \\
\hline Mta & Active Tidal Flat & & DISTURBANCE \\
\hline Mti & Inactive Tidal Flat & Hch & Clearing, habitat enhancement \\
\hline Hf & Fill and Embankments (undifferentiated) & Hen & Clearing, no soil removal \\
\hline $\mathrm{He}$ & Excavations & Hes & Clearing, soil removal \\
\hline Wldim & Deep Isolated Lakes, Morainal & Ht & Undifferentiated Trail \\
\hline Wlsim & Shallow Isolated Ponds, Morainal & Hfg & Gravel Fill \\
\hline Wrun & Upper Perennial River, Non-glacial & Hfgr & Gravel Road \\
\hline Wrlg & Lower Perennial River, Glacial & Hfga & Gravel Airstrip \\
\hline Wrln & Lower Perennial River, Non-glacial & Hfgp & Gravel Pad \\
\hline Welt & Tidal Ponds & Hfgrp & Paved Road \\
\hline Wert & Tidal River & Hfgap & Paved Airstrip \\
\hline Wmn & Nearshore Water & $\mathrm{Hf}$ & Undifferentiated Fill \\
\hline \multirow[t]{7}{*}{ Wh } & Man-made Waterbodies & Het & Trench \\
\hline & & Hwd & Ditch \\
\hline & & Hep & Mine Pit \\
\hline & & Hwe & Water-filled excavation \\
\hline & & $\mathrm{Hfb}$ & Berms, Spoil Piles \\
\hline & & $\mathrm{He}$ & Excavation/Pits (undifferentiated) \\
\hline & & $\mathrm{DC}$ & Disturbance Complex \\
\hline
\end{tabular}


Table 2. Comparison of systems for differentiating ecosystems at various scales.

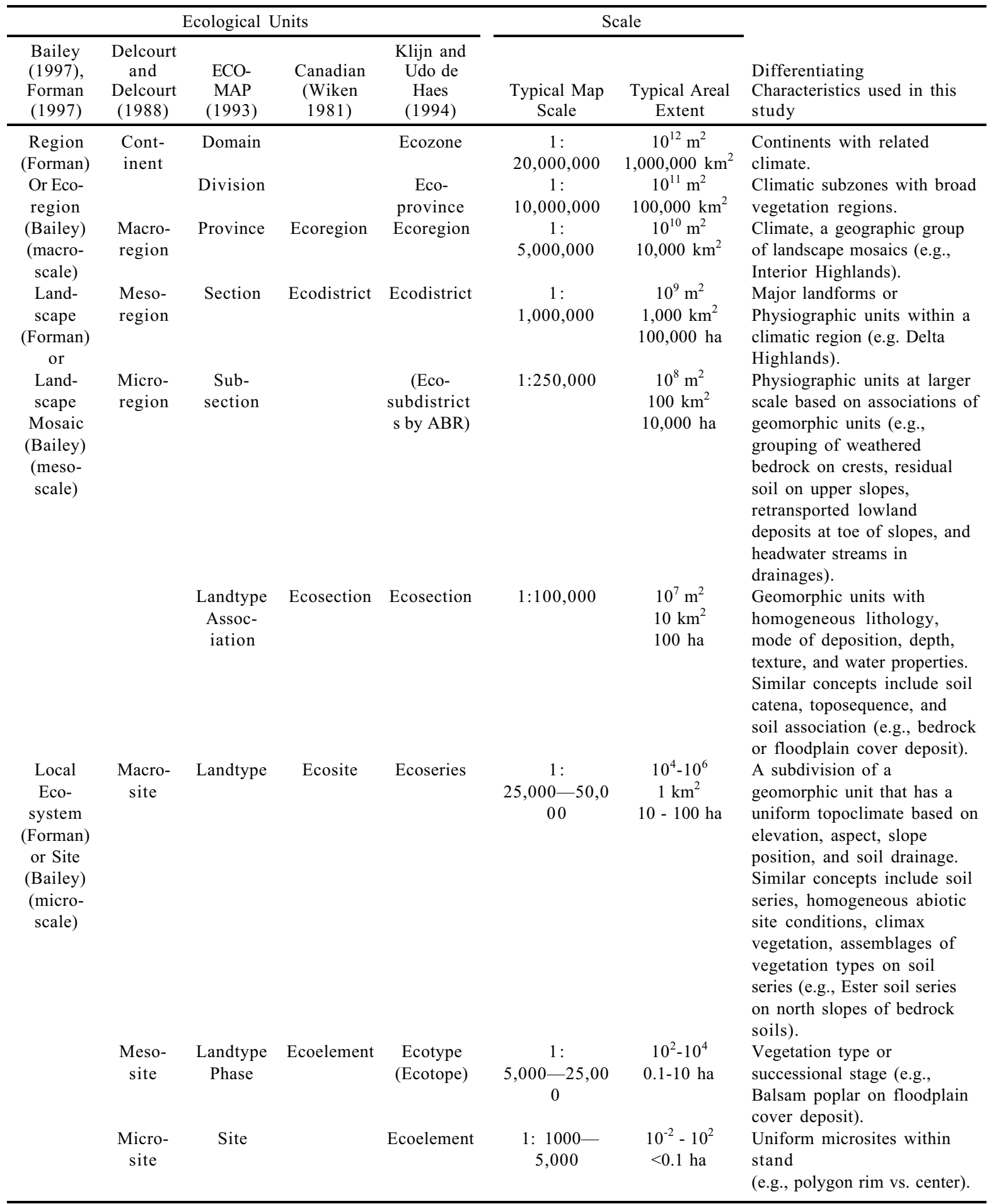


Table 3. Classification and description of geomorphic units within Fort Richardson, southcentral Alaska, 2001. Descriptions derived in part from ADGGS (1983) and Hunter et al. (2000).

\begin{tabular}{ll}
\hline Geomorphic Unit & Description \\
\hline $\begin{array}{l}\text { Metamorphic } \\
\text { Bedrock (N) }\end{array}$ & $\begin{array}{l}\text { Undifferentiated metamorphic rocks modified from other rocks through changes in chemical environment, } \\
\text { temperature or pressure. In the Chugach Mountains, the rocks are dominated by the McHugh Complex, } \\
\text { comprised of a sequence of metamorphosed graywacke, argillite, phyllite, and conglomerate graywacke. } \\
\text { Also included are small areas with a metavolcanic sequence of greenstone, metachert, and argillite. }\end{array}$ \\
$\begin{array}{ll}\text { Younger Rock } \\
\text { Glacier (Cgy) }\end{array}$ & $\begin{array}{l}\text { A tongue-shaped mass of angular rock fragments formed by slow movement of an ice core or interstitial } \\
\text { ice. Found at the base of an extensive talus-producing surfaces such as steep valley wall or recent } \\
\text { moraines. Surface is dominated by cobbles and boulders, and generally lacks vegetation. At depth, fine- } \\
\text { grained material may be present to form coarse, rubbly, massive, and poorly sorted diamicton. Permafrost }\end{array}$ \\
$\begin{array}{l}\text { is present. } \\
\text { Solifluction } \\
\text { Deposits (Cs) (not } \\
\text { mapped) }\end{array}$ & $\begin{array}{l}\text { Saturated soil material and rock fragments formed by downslope, viscous flow of the active layer. The unit } \\
\text { Talus Bluff (Ctb) }\end{array}$ \\
$\begin{array}{l}\text { Loose, thin accumulations on the steep slopes of coastal and stream bluffs derived from adjacent, upslope } \\
\text { surficial deposits. Chiefly diamicton, consisting of pebbly silt and sand, some clay, cobbles, and boulders } \\
\text { with variable amounts of organic material; massive to poorly bedded, poorly sorted. Usually vegetated. }\end{array}$ \\
$\begin{array}{l}\text { Angular rubble or rock fragments that have accumulated by gravity at the base of cliffs and steep slopes. } \\
\text { The talus makes a cone-shaped deposit, usually at the base of chutes. Usually unstable and partially }\end{array}$ \\
$\begin{array}{l}\text { vegetated. } \\
\text { Landslide Deposits }\end{array}$ \\
$\begin{array}{l}\text { Irregular to hummocky deposits at the base of steep slopes resulting from slope failure and deposition of } \\
\text { loose material. Includes a wide variety of materials, chiefly diamicton, with lesser amounts of gravelly silt, } \\
\text { sand, organic matter, rubble, and some large masses of bedrock. }\end{array}$
\end{tabular}
sand, organic matter, rubble, and some large masses of bedrock.

Hillside Colluvium Apron-like deposits of loose sandy to rubbly diamicton derived directly from weathering of bedrock $(\mathrm{Ctm}) \quad$ upslope and usually including some sheet-wash deposits. Thickness probably $0.5-5 \mathrm{~m}$, thicker on lower slopes. Somewhat unstable, but usually vegetated. Occurs on mountain slopes downslope of bedrock.

Loess (El) (not mapped)

Younger Moraine (Gmy)

Older Moraine (Gmo)

Kame Deposits (GFk)

Glaciomarine

Deposits (MG)

Glaciofluvial Outwash (GFo)

Glaciofluvial Channel (GFc)
Windblown silt typically occurring as a thin cap on older deposits. The massive silt lacks horizontal stratification and coarse fragments. Deposit must be $>40 \mathrm{~cm}$ thick. On Fort Richardson, the loess cap usually varied from 20 to $40 \mathrm{~cm}$, rarely to $60 \mathrm{~cm}$.

Relatively young moraines with steeper knob and basin topography with a poorly integrated drainage network. The deposits are composed of glacial till material deposited at the terminal or lateral margins of a glacier that has since retreated or disappeared. Younger moraines have less basin filling. Sediments are highly variable ranging from poorly sorted sand and subangular gravel with some boulders to sorted coarser subrounded material.

Similar to above except older moraines have subdued topography with broader knobs and swales and more integrated drainage network. Soils show more leaching and horizon development.

Steep hills and hummocky areas with occasional depressions formed by running water within a glacier during early stages of stagnation and modified during ice melt out. The meltwater detritus occurs as fillings or as partial fillings of depressions against valley walls and within glacial crevasses, or as part of steep-sided alluvial fans deposited against the margin of an ice sheet. A kame may form as terraces, conical hills, or short irregular ridges. Deposits are comprised of moderately to well bedded cobbles, gravel, and sand.

Complex areas where marine, glacial, and lacustrine processes intergraded in an environment of fluctuating shallow marine or estuarine waters. Deposits include submarine till sheets, ice-rafted materials, and quiescent water deposits. The deposits typically comprised of silt and clay with some sand, but few boulders. Topography is generally flat to gently sloping, but marked locally by small subdued hills or minor surface irregularities. Includes the Bootlegger Cove Formation.

Deposits formed by meltwater streams beyond the terminal glacial margin. It includes outwash fans, deltas, aprons, and valley trains deposited by an earlier glacial period. Deposits are well-stratified, moderately to well-sorted, clean-wash bedload sand and gravel with some boulders. They lack significant accumulations of fine-grained cover deposits, but can have organic matter $(>10 \mathrm{~cm})$ at the surface or have well-developed A horizons.

Flat to gently sloping areas formed by meltwater streams in narrow channels during glacial retreat. Deposits are comprised of well-bedded and sorted gravel and sand. The surface often includes fine-grained material with thin organic accumulations. Differentiated from outwash by its close association with glacial deposits behind the front of a glacial moraine. 
Table 3 (cont'd). Classification and description of geomorphic units within Fort Richardson, south-central Alaska, 2001. Descriptions derived in part from ADGGS (1983) and Hunter et al. (2000).

\begin{tabular}{|c|c|}
\hline Unit & n \\
\hline $\begin{array}{l}\text { acustrine (L) (not } \\
\text { apped) }\end{array}$ & $\begin{array}{l}\text { Silt and clay materials deposited in both glacial and non-glacial lakes. Lake sediments generally are well } \\
\text { stratified into very thin laminations, but may also include coarse-grained sediments associated with } \\
\text { shorelines and fluvial sediments in deltas and fans. }\end{array}$ \\
\hline $\begin{array}{l}\text { Glaciolacustrine } \\
\text { Deposits (GL) }\end{array}$ & $\begin{array}{l}\text { Glacial lake deposits formed within irregular morainal topography, swell and swale topography of till } \\
\text { plains, behind terminal or lateral moraines, in kettles on outwash plains, in ice-marginal areas, or by } \\
\text { damming behind glacial lobes. Deposits are well to somewhat poorly sorted and comprised of interbedded } \\
\text { clay, silt, and sand, and may include some gravel and diamicton. Topography is flat to gently sloping. }\end{array}$ \\
\hline Bogs $(\mathrm{Ob})$ & $\begin{array}{l}\text { Ombrotrophic bogs with thick }(>40 \mathrm{~cm}) \text { organic matter accumulations developed in basins with essentially } \\
\text { closed drainage receiving their water from precipitation and immediate surroundings. The surface is flat } \\
\text { and the water table is near the surface. Organic matter is dominated by fibric peat of Sphagnum mosses and } \\
\text { ericaceous woody material, but may be underlain by sedge peat. Minor occurrences of minerotrophic } \\
\text { peatlands (fens) were also included in this class. }\end{array}$ \\
\hline $\begin{array}{l}\text { Headwater } \\
\text { Moderately Steep } \\
\text { Floodplain (Fhm) }\end{array}$ & $\begin{array}{l}\text { Small, shallow deposits formed in the upland headwaters of small creeks with moderately steep }(2-6 \%) \\
\text { stream gradients. Sediment deposition processes are limited and channel banks are comprised of boulder } \\
\text { and bedrock materials that limit floodplain development. Channel and overbank deposits are not } \\
\text { differentiated. }\end{array}$ \\
\hline $\begin{array}{l}\text { Headwater Lowland } \\
\text { loodplain (Fhl) }\end{array}$ & $\begin{array}{l}\text { Small, shallow deposits formed in the headwaters of small creeks in lowland areas. The low stream } \\
\text { gradients }(<2 \%) \text { are associated with "bog" streams and places where small streams originating from upland } \\
\text { areas cross low-lying flat areas. Deposits usually range from gravelly sand to fine-grained and organic-rich }\end{array}$ \\
\hline
\end{tabular}

Alluvial Fan (Ff) Gently sloping cone-shaped deposit of alluvium formed where a stream extends onto a relatively level plain, such as where streams issue from mountains onto lowland. Alluvial fans are comprised predominantly of coarse-grained materials, but also have varying quantities of silt.

Old Alluvial Fan Similar to above, except material was deposited by early fluvial regime that no longer exists. Thus, (Ffo) gravelly deposits have substantial organic layers at the surface or well developed A horizons, indicating a long period since last depositional event.

Alluvial Terrace $(\mathrm{Ft})$ Relatively flat surfaces resulting from the dissection of former floodplain areas. Terraces are old and are not subject to flooding under the current regime. Deposits consist of gravelly sand, sand, silty sand, and peat. Deposits usually are overlain by eolian silt and sand and have moderately thick organic horizons.

Alluvial Plain Undifferentiated glacial and non-glacial granular deposits in areas where depositional processes are Deposits (FGp) transitional between glaciofluvial and fluvial environments, or where deposits are of unknown origin. Deposits occur across a broad, flat to gently sloping plain. Materials generally comprised of poorly stratified gravel and sand. On Ft. Richardson, this includes the Mountain View alluvial fan (also referred to as "Naptowne outwash") deposited during the late Pleistocene that forms terraces from the Chugach Mountains to downtown Anchorage.

Meander Active Lateral accretion deposits formed in meandering channels that wind freely in regular to irregular, wellChannel Deposits developed, S-shaped curves. Channels range from highly sinuous to only slightly meandering. Riverbed (Fmra) material can range from gravel to gravelly sand and lateral accretion deposits along point bars typically are sandier.

Meander Inactive Mixed lateral and vertical accretion deposits in inactive ("high water" or "cut-off") channels that are Channel Deposits flooded only during high-water events. Riverbed material often has a thin layer of fine-grained material (Fmri) over the coarse channel deposits and surface is usually vegetated.

Meander Lateral accretion deposits of a meander floodplain that no longer is associated with the present fluvial Abandoned Channelregime or where flooding is sufficiently infrequent that fluvial sediments form a negligible component of Deposits (Fmrb) surface material. Surface materials are dominated by gravel or sand and lack fine-grained overbank deposits. Lack of flooding is indicated by the presence of an A horizon indicative of long, undisturbed soil development or by the presence of organic layers in $>90 \%$ of the top $40 \mathrm{~cm}$.

Meander Active Vertical accretion deposits on low portions of the overbank environment in close proximity to the Overbank Deposits meandering river channels. The deposits are comprised of silts and fine sands that have a laminar, (Fmoa) interbedded structure formed by changes in velocity and deposition during waxing and waning floods. Frequent flooding and sedimentation prevents organic matter accumulation. Fine-grained material must be $>40 \mathrm{~cm}$ thick and organic layers comprise less than $10 \%$ of the thickness. 
Table 3 (cont'd).

\begin{tabular}{|c|c|}
\hline Geomorphic Unit & Description \\
\hline $\begin{array}{l}\text { Meander Inactive } \\
\text { Overbank Deposits } \\
\text { (Fmoi) }\end{array}$ & $\begin{array}{l}\text { Vertical accretion deposits formed on higher portions of the overbank environment in close proximity to } \\
\text { meandering river channels. Areas are subject to infrequent flooding (approx. every } 5-25 \text { years). Comprised } \\
\text { of interbedded organics, silts and fine sands. Deposit is }>40 \mathrm{~cm} \text { thick and organic layers comprise } 10-90 \% \\
\text { of the top } 40 \mathrm{~cm} \text {. }\end{array}$ \\
\hline $\begin{array}{l}\text { Meander } \\
\text { Abandoned } \\
\text { Overbank Deposits } \\
\text { (Fmob) }\end{array}$ & $\begin{array}{l}\text { Vertical accretion deposits of a meandering floodplain that no longer are associated with the present } \\
\text { fluvial regime or where flooding is sufficiently infrequent that fluvial sediments form a negligible } \\
\text { component of surface material. Surface materials often include a mixture of fluvial, eolian, and organic } \\
\text { materials, but typically are highly organic. The deposit is }>40 \mathrm{~cm} \text { thick, and organic layers comprise }>90 \% \\
\text { of the top } 40 \mathrm{~cm} \text {. Organic deposits }(>40 \mathrm{~cm}) \text { are difficult to distinguish from this unit, so this unit often } \\
\text { includes thick peat accumulations. }\end{array}$ \\
\hline $\begin{array}{l}\text { Active Tidal Flat } \\
\text { (Mta) }\end{array}$ & $\begin{array}{l}\text { Areas of nearly flat, barren mud or fine sand that are periodically inundated by tidal waters. Tidal flats } \\
\text { occur on seaward margins of deltaic estuaries, leeward portions of bays and inlets, and at mouths of rivers. } \\
\text { Tidal flats frequently are associated with lagoons and estuaries and may vary widely in salinity, } \\
\text { depending on how exposed the flat is to salt-water incursion and the rate of influx of fresh water. }\end{array}$ \\
\hline $\begin{array}{l}\text { Inactive Tidal Flat } \\
\text { (Mti) }\end{array}$ & $\begin{array}{l}\text { Similar to active tidal flats, but flooded only by during larger storm events. The sediments are only } \\
\text { slightly brackish and the surface usually is well vegetated. The silty sediments usually have thin organic } \\
\text { horizons. }\end{array}$ \\
\hline $\begin{array}{l}\text { Fill and } \\
\text { Embankments, } \\
\text { undifferentiated } \\
\text { (Hf) }\end{array}$ & $\begin{array}{l}\text { All forms of man-made fill or embankment materials, including road and foundation embankments, dikes, } \\
\text { and other artificial earth fills. Fill is often comprised largely of sand and gravel. }\end{array}$ \\
\hline Excavations $(\mathrm{He})$ & $\begin{array}{l}\text { Man-made excavations, including large cuts, gravel removal sites, and other mine pits. Remaining } \\
\text { unconsolidated or bedrock material is highly variable depending on local geology. }\end{array}$ \\
\hline $\begin{array}{l}\text { Deep Isolated } \\
\text { Lakes, Morainal } \\
\text { (Wldim) }\end{array}$ & $\begin{array}{l}\text { Deep }(>1.5 \mathrm{~m}) \text { kettle ponds and lakes that do not freeze to the bottom during winter. The lakes do not have } \\
\text { distinct outlets and are not connected to rivers. The lakes develop from the melting of glacial ice in } \\
\text { moraines and typically have rocky bottoms. }\end{array}$ \\
\hline $\begin{array}{l}\text { Shallow Isolated } \\
\text { Ponds, Morainal } \\
\text { (Wlsim) }\end{array}$ & $\begin{array}{l}\text { Shallow }(>1.5 \mathrm{~m}) \text { kettle ponds and lakes that often freeze to the bottom during winter. The lakes do not } \\
\text { have distinct outlets and are not connected to rivers. The lakes develop from the melting of glacial ice in } \\
\text { moraines and typically have rocky bottoms. }\end{array}$ \\
\hline $\begin{array}{l}\text { Upper Perennial } \\
\text { River, Non-glacial } \\
\text { (Wrun) }\end{array}$ & $\begin{array}{l}\text { Permanently flooded channels of freshwater rivers where the gradient is relatively high and discharge and } \\
\text { water quality are not affected by glacial meltwater. Rivers generally experience peak flooding during } \\
\text { spring breakup and late summer, and lowest water levels during mid-summer. Suspended sediment } \\
\text { concentrations are low. }\end{array}$ \\
\hline $\begin{array}{l}\text { Lower Perennial } \\
\text { River, Glacial } \\
\text { (Wrlg) }\end{array}$ & $\begin{array}{l}\text { Permanently flooded channels of freshwater rivers where the gradient is relatively low, and discharge and } \\
\text { water quality are affected by glacial meltwater. River water may appear discoloured from high } \\
\text { concentrations of suspended sediments during mid-summer. Rivers experience peak flooding during mid- } \\
\text { summer. }\end{array}$ \\
\hline $\begin{array}{l}\text { Lower Perennial } \\
\text { River, Non-glacial } \\
\text { (Wrln) }\end{array}$ & $\begin{array}{l}\text { Permanently flooded channels of freshwater rivers where the gradient is relatively low and discharge and } \\
\text { water quality are not affected by glacial meltwater. Water sources are not differentiated and can include } \\
\text { surface runoff and deep groundwater. Rivers generally experience peak flooding during spring breakup } \\
\text { and late summer and lowest water levels during mid-summer. Suspended sediment concentrations are low. }\end{array}$ \\
\hline Tidal Ponds (Welt) & $\begin{array}{l}\text { Coastal ponds that are flooded periodically with saltwater during high tides or storm surges. Salinity } \\
\text { levels often are increased by subsequent evaporation of impounded saline water. The substrate frequently } \\
\text { is silt with some clay and fine sand, and occasionally contains peat. }\end{array}$ \\
\hline Tidal River (Wert) & $\begin{array}{l}\text { Permanently flooded channels of the Eagle River Flats that are affected by daily tidal fluctuations and } \\
\text { have correspondingly variable salinity. The channels generally experience peak flooding during spring } \\
\text { breakup and lowest water levels during mid-summer. During winter, deeper channels can have elevated } \\
\text { salinity levels. }\end{array}$ \\
\hline $\begin{array}{l}\text { Nearshore Water } \\
(\text { Wmn) }\end{array}$ & $\begin{array}{l}\text { Shallow estuaries, lagoons, and embayments along Cook Inlet. Winds, tides, river discharge, and sea ice } \\
\text { create dynamic changes in physical and chemical characteristics. Tidal range is very large }(9-11 \mathrm{~m}) \text { and } \\
\text { storm surges produced by winds may raise sea level several meters. Bottom sediments are mostly } \\
\text { unconsolidated mud. During winter the water is covered with ice floes. }\end{array}$ \\
\hline $\begin{array}{l}\text { Man-made } \\
\text { Waterbodies (Wh) }\end{array}$ & Man-made waterbodies including impoundments, water-filled pits, and ditches. \\
\hline
\end{tabular}




\section{Ecosystems and landscapes}

Ecotype (local ecosystem) classes were developed to integrate the physiography, soil texture, geomorphic units, slope position, drainage, vegetation structure, and vegetation composition (plant association) of small, uniform areas. Classification of ecotypes was accomplished in three general steps: (1) individually classifying the detailed ground descriptions, (2) developing graphic profiles of ecosystem components along transects (toposequences) to identify ecological relationships among terrain features, and (3) deriving a reduced set of ecotypes by identifying the most common relationships and central tendencies. In the development of these classes, we tried to use ecological characteristics (primarily geomorphology, surface form, and vegetation structure) that could be interpreted from aerial photographs. We also developed a nomenclature for ecotypes that explicitly relates ecological characteristics in a terminology that can be easily understood.

To reduce the number of different ecotypes and the complexity of highly variable ecological characteristics, it was necessary to aggregate detailed characteristics described in the field (e.g., soil stratigraphy and vegetation composition). For each component, we used a hierarchical approach to aggregation (Fig. 3). For geomorphology, we aggregated classes, textures, layers, and lithofacies into geomorphic units (architectural elements) using the approaches of Miall (1985). Geomorphic units were assigned to physiographic settings based on their erosional or depositional processes (Appendices A and B). Surface forms were simplified into a reduced set of slope elements (i.e., crest, upper slope, lower slope, toe, flat). For vegetation, we used the structural levels (III) of the Alaska Vegetation Classification (Viereck et al. 1992) because they are more readily identifiable on aerial photography than are floristic associations.

Common relationships among ecosystem components were identified by visual examination of graphic profiles, use of contingency tables, and detrended correspondence analysis (DCA). Graphical presentation of topographic sequences provided an overview of successional relationships among vegetation classes and landscape features. The contingency tables sorted plots by climate zone, physiography, texture, geomorphic unit, drainage, and vegetation type. From these tables, common associations were identified and uncommon associations either were lumped with those having simi-

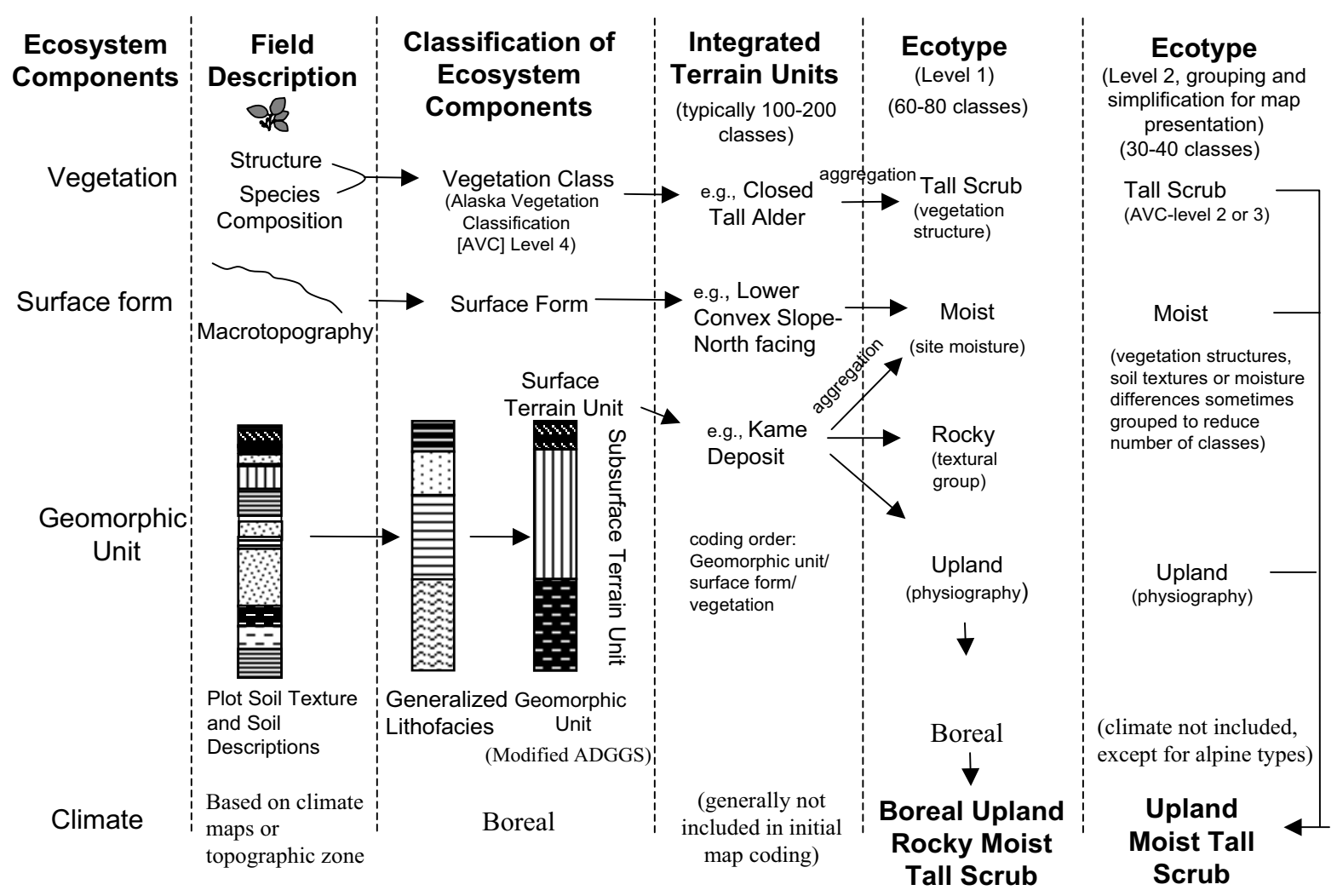

Figure 3. System of hierarchically classifying ecosystem components into integrated terrain units (ITU) and ecotypes. 
lar characteristics or excluded as unusual (outliers). The DCA analysis further emphasized plots that grouped together as a result of ecological similarities and helped identify unusual plots or outliers. Our goal was to identify strong relationships that could be used for prediction and mapping, while avoiding the creation of unnecessary classes that might add confusion and decrease accuracy.

Ecotype names were based on the simplified ecosystem components. For example, a full ecotype name for an individual plot would be Boreal Upland Rocky Acidic Moist Mixed Forest, based on climatic, physiographic, textural, chemical ( $\mathrm{pH}$ or salinity), hydrologic (moisture), and vegetative components, respectively. We started by reducing the ecotype name to a sequential coding of physiography, dominant soil texture, soil moisture, and vegetation structure. Because this generated a large number of specific ecotypes (over 70) from the 231 field plots, we aggregated some similar ecotypes into a reduced set of 46. Some textural classes were grouped (e.g., rocky and loamy) because the vegetation classes were similar, and some similar vegetation structures (e.g., open and closed black spruce) were grouped because species composition was similar. These groupings were based on the most frequent associations identified during the cross-tabulation of components. Overall, we tried to balance the need to adequately differentiate ecological characteristics with the need to minimize the number of classes for management purposes. Other users may wish to group characteristics in different ways for their own individual purposes. The field data can be reclassified and analyzed by regrouping ecological characteristics and applying the new organization to the plot database (Appendices $\mathrm{A}$ and $\mathrm{B})$.

The human-modified ecotypes were classified using a different approach. Because clearings, roads, and other forms of human modification occur across the landscape, a substantial number of additional ecotypes would be required to classify these areas at the same level of detail. In an effort to consolidate classes, we assigned all identifiably human-affected areas to the Human Modified ecotype. We further reduced the number of classes by consolidating the vegetation descriptions for these areas into generic vegetation structures. For example, a class that might properly be called Lowland Gravelly Moist Low Scrub Disturbed was classified as Human-Modified Scrub.

For landscape-level classification of ecosystems, geomorphic and physiographic criteria were used for differentiation (Table 2). Ecosections were differentiated based on geomorphic patterns and processes and named after soil textural and physiographic groups (e.g., Gravelly Lowland, Loamy Coast). Classification of ecodistricts and ecosubdistricts was based on general physiographic characteristics that were related to associations of geomorphic units. Thus, each ecodistrict will have several ecosections. Because each ecodistrict is unique, the name for each area was based on a general physiographic descriptor (e.g., lowland or highland) and a prominent geographic feature (e.g., nearby creek or mountain).

\section{Mapping}

Ecosystems were mapped at three scales: ecotype (1:20,000), ecosection $(1: 100,000)$, and ecodistrict and ecosubdistrict $(1: 250,000)$. The ecotypes and ecodistricts involved independent delineations, while the ecosection map was created from the physiographic and textural classes of the ecotype map.

\section{Ecological components}

Individual ecological components were mapped simultaneously at 1:20,000 as compound codes called integrated terrain units (ITUs). ITUs were mapped by assigning a four-parameter code to each polygon describing geomorphology, surface form, vegetation, and disturbance type. Delineation was done on-screen using a black and white, orthorectified, photo mosaic developed from 1997 aerial photography and produced by AeroMap, Inc. (Anchorage, Alaska). Thus, the mosaic provided high-quality geometric control for the linework. Because black and white photography is not useful for differentiating some vegetation characteristics, we relied on other imagery during the photo-interpretation process. For on-screen use we created a mosaic of 1:60,000 false color infrared (CIR) photography taken in August 1984 and georeferenced it to the black and white orthophoto base map. We also acquired a Landsat 7 Thematic Mapper (TM) image (9 August 2000, Path 69, Row 17) orthorectified by RADARSAT, Inc. We also referred to hard copies of 1:12,000 CIR photography taken on 20 August 1995 . The minimum mapping size for polygons was $\sim 0.5$ ha. To avoid use of complex associations, a map class or polygon was allowed to include up to $30 \%$ of another class. Thematic maps were produced for each individual ITU component: geomorphology, surface form, vegetation, and disturbance class.

\section{Ecosystems and landscapes}

Ecotypes and ecosections were derived by aggregating ITU codes. After mapping was completed, an ecotype class was assigned in the map database to each ITU code. Mapping generated many combinations of characteristics (ITU codes) that did not occur in the plot data. We assigned ecotypes to these ITUs using basic relationships of physiography, soil texture, and 
vegetation structure that were defined by the field data and vegetation analysis (Appendix C).

Ecotypes were aggregated, based on physiography and soil texture, to produce an ecosection map $(\sim 1: 100,000)$ showing associations of related geomorphic types. At the ecosection level, the Human Modified class was restricted to sites where soils were disturbed. Consequently, only polygons identified as Excavation or Fill were included in this category.

Ecodistricts were delineated on-screen over a 1:100,000-scale view of the Landsat TM image as a separate effort and not by aggregating lower-level units. Where possible, we attempted to make our boundaries consistent with those used in previous studies, including maps of land resource areas used in the exploratory soil survey of Alaska (Rieger et al. 1979), glacial geology (Reger and Updike 1983), bedrock geology (Nokleberg and Plafker 1994, Plafker et al. 1994), and the ecoregions of Alaska (Nowacki et al. 2002). On the eastern edge of the map we joined our ecological units with those of Davidson (1997). Ecodistrict mapping encompassed a larger area than ecotype and ecosections maps. By extending the ecodistrict map north and west of Knik Arm and south along the Chugach Mountains, regional relationships of the landscape $(1: 250,000)$ were made more apparent.

\section{RESULTS AND DISCUSSION}

\section{Ecosystem components Climate}

The climate of Anchorage is transitional between maritime (warm winters, high precipitation, and frequent high winds) and interior-continental (cold winters, low precipitation, and generally light winds). The climate is strongly affected by the Chugach Range, which acts as a barrier to the influx of warm, moist air from the Gulf of Alaska. Precipitation in Anchorage is only $10-15 \%$ of that measured on the Gulf of Alaska side of the Chugach Range. To the north, the Alaska Range acts as a barrier to the influx of polar air masses from the interior. Consequently, summers are cooler and winters warmer than at more inland stations.

Based on long-term climatic records (1952-2000) for Anchorage (elevation $34 \mathrm{~m}$ ), the mean annual temperature is $2.2^{\circ} \mathrm{C}\left(35.9^{\circ} \mathrm{F}\right)$, with mean monthly temperatures ranging from $-9.6^{\circ} \mathrm{C}$ in January to $14.7^{\circ} \mathrm{C}$ in July (Fig. 4). Daily extremes over the period of record ranged from $-37^{\circ} \mathrm{C}$ to $29^{\circ} \mathrm{C}$. The thawing season lasts approximately 200 days beginning in early April and ending in late October. Mean annual precipitation is $400 \mathrm{~mm}$ (15.7 in.), with mean monthly precipitation ranging from $14 \mathrm{~mm}$ in April to $69 \mathrm{~mm}$ in August. The highest daily precipitation ever recorded was $70 \mathrm{~mm}$. About half the precipitation falls as snow.

The climate in the Chugach Mountains is greatly affected by elevation, although few data are available to evaluate climatic gradients. Some climatic data (1949-1977) are available for Eklutna Lake (approximate elevation $300 \mathrm{~m}$ ), adjacent to Fort Richardson. At that station, mean annual temperature is $-0.3^{\circ} \mathrm{C}$ $\left(31.5^{\circ} \mathrm{F}\right)$, with mean monthly temperatures ranging from $-14.8^{\circ} \mathrm{C}$ in January to $12.8^{\circ} \mathrm{C}$ in July. Mean annual precipitation is $302 \mathrm{~mm}$ (11.9 in.), with mean monthly precipitation ranging from $12 \mathrm{~mm}$ in February to $42 \mathrm{~mm}$

Anchorage WSCMO AP $(33.5 \mathrm{~m})$

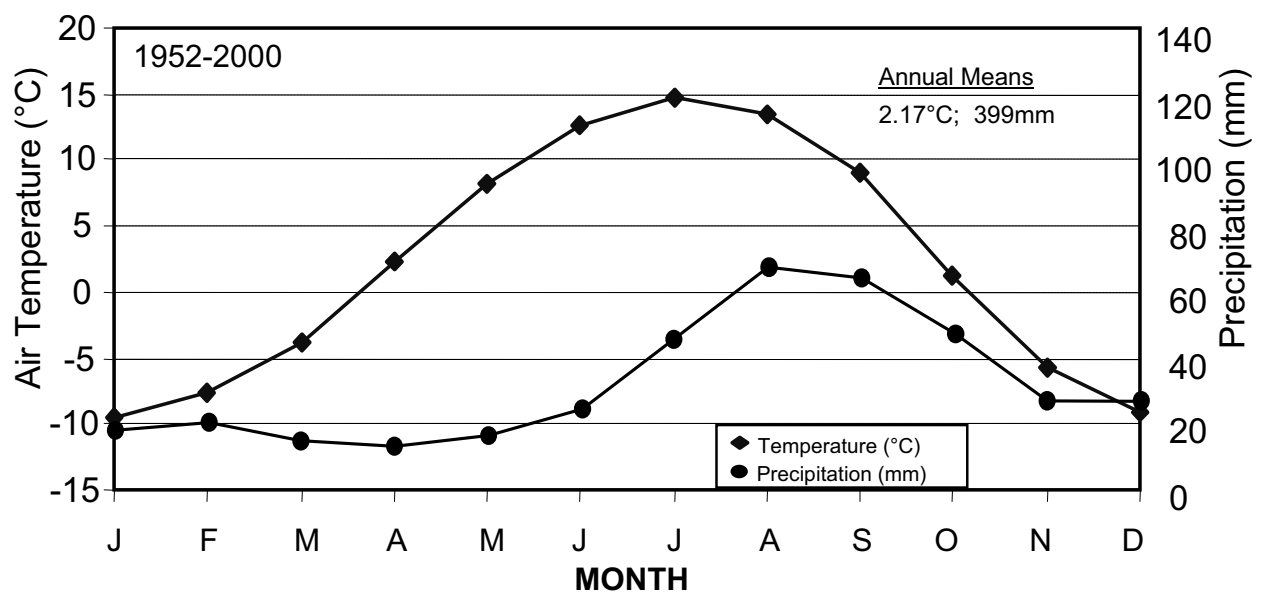

Figure 4. Climate diagram (mean monthly temperature and precipitation) for the Anchorage station near Fort Richardson, south-central Alaska. 
in August. Overall, the climate at Eklutna Lake is colder and drier than at the Anchorage station.

No climatic data are available for the alpine areas of Fort Richardson. In the Yukon Training Area on Fort Wainwright in interior Alaska, the summer lapse rate with elevation was $-3.7^{\circ} \mathrm{C}$ per $1000 \mathrm{~m}$, indicating that summer temperatures in the alpine zone were considerably lower (Jorgenson et al. 1999).

\section{Tectonics and bedrock geology}

Fort Richardson covers two broad lithotectonic terranes, the Chugach terrane with its rugged mountains, and the Cenozoic surficial deposits in the lowland area (Plafker et al. 1994, Winkler et al. 1984) (Fig. 5). The Chugach terrane is associated with the mountain-building processes arising from the subduction of the $\mathrm{Pa}$ cific Plate under the North American Plate. Subduction in this area was most active during the Early Jurassic through the Late Cretaceous, along the large Border Ranges fault system that separates the terranes. Near the study area, the Chugach terrane is dominated by the Melange assemblage of Lower Cretaceous and Upper Jurassic rocks, including flysch, greenstone, limestone, chert, granodiorite, glaucophane-bearing greenschist, and layered gabbro and serpentenite. The main portion of the Chugach terrane outside the study area is dominated by Cretaceous and Upper Jurassic rocks that include graywacke, slate, argillite, minor conglomerate, volcanic detritus, and interbedded mafic volcanic rocks. Within Fort Richardson, the geology is dominated by relatively uniform graywacke. A small outcrop of ultramafic rocks occurs on the hillslope area between Ship Creek and Eagle River.

Tectonics and bedrock geology are important ecological factors, because mountain ranges affect movement of air masses (discussed under climate) and bedrock types affect the chemistry and acidity of soils (Bailey 1996). The noncarbonate metamorphic rocks that predominate in the study area tend to weather into acidic soils.

\section{Geomorphology}

Field surveys identified 38 geomorphic units within the study area, including 34 terrestrial units and four waterbody classes (Table 3, Appendix 1). During mapping, 30 terrestrial and nine waterbody classes were used (Table 4, Fig. 6). Two units were not mapped: loess because it occurred as a surface layer too thin or discontinuous to map, and lacustrine because it was overlain by organic deposits or water. Additional classes mapped but not described from ground data included Tidal Flats, Tidal Ponds, and Nearshore Water. Note that mapping was based on the geomorphic unit at the surface, although underlying stratigraphic units that are

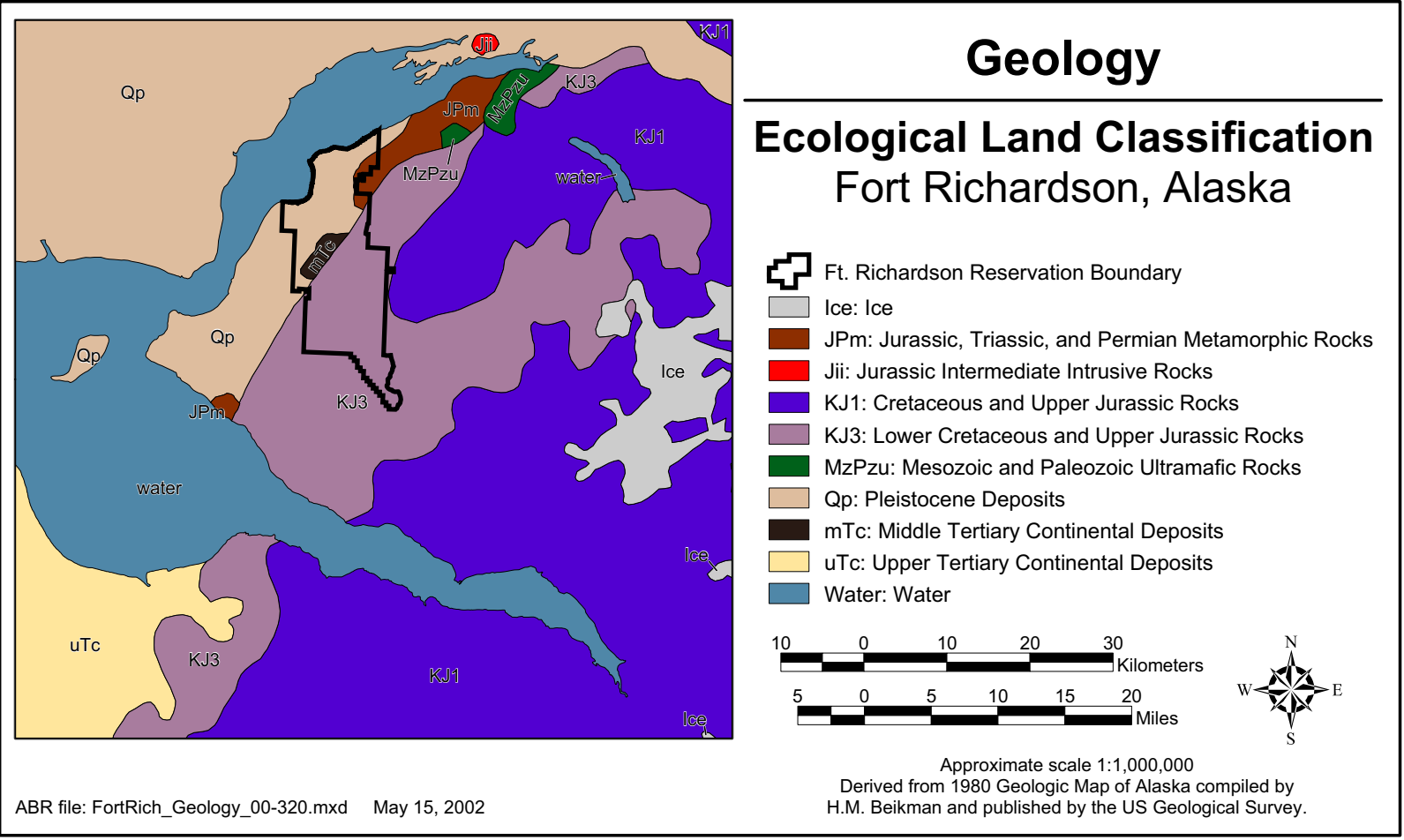

Figure 5. Map of the geology of the region surrounding Fort Richardson, south-central Alaska, 2001. 
Table 4. Areal extent of geomorphic units and surface forms mapped on Fort Richardson, southcentral Alaska, 2001.

\begin{tabular}{|c|c|c|c|c|c|c|c|}
\hline \multirow[b]{2}{*}{ Geomorphic Unit } & \multicolumn{3}{|c|}{ Area } & \multirow[b]{2}{*}{ Surface Form } & \multicolumn{3}{|c|}{ Area } \\
\hline & Acre & ha & $\%$ & & Acre & ha & $\%$ \\
\hline Metamorphic Bedrock & 1843 & 746 & 3.0 & Crest & 1850 & 749 & 3.0 \\
\hline Younger Rock Glacier & 113 & 46 & 0.2 & Bluff & 1377 & 557 & 2.2 \\
\hline Talus Bluff & 1322 & 535 & 2.1 & Streambanks & 17 & 7 & $<0.1$ \\
\hline Talus Cone & 390 & 158 & 0.6 & Upper Slope, north facing & 4507 & 1824 & 7.3 \\
\hline Landslide Deposits & 170 & 69 & 0.3 & Upper Slope, south facing & 5075 & 2054 & 8.2 \\
\hline Hillside Colluvium & 13,930 & 5638 & 22.5 & Upper Slope, east or west facing & 6187 & 2504 & 10.0 \\
\hline Younger Moraine & 1321 & 535 & 2.1 & Upper Slope, concave & 255 & 103 & 0.4 \\
\hline Older Moraine & 15,500 & 6273 & 25.0 & Lower Slope & 13 & 5 & 0.0 \\
\hline Kame Deposits & 8534 & 3454 & 13.8 & Lower Slope, north facing & 1540 & 623 & 2.5 \\
\hline Glaciomarine Deposits & 523 & 212 & 0.8 & Lower Slope, south facing & 2154 & 872 & 3.5 \\
\hline Glaciofluvial Outwash & 134 & 54 & 0.2 & Lower Slope, east or west facing & 4142 & 1676 & 6.7 \\
\hline Glaciofluvial Channel & 3769 & 1525 & 6.1 & Lower Slope, concave & 624 & 253 & 1.0 \\
\hline Glaciolacustrine Deposits & 33 & 13 & 0.1 & Lower Slope, convex & 4 & 2 & $<0.1$ \\
\hline Bogs & 1508 & 610 & 2.4 & Moraine Complex, undulating & 16289 & 6592 & 26.3 \\
\hline Headwater Moderately Steep & 314 & 127 & 0.5 & Flat or Fluvial Related & 13296 & 5381 & 21.4 \\
\hline Headwater Lowland Floodplain & 153 & 62 & 0.2 & Channel, Swale or Gut & 229 & 93 & 0.4 \\
\hline Alluvial Fan & 52 & 21 & 0.1 & Interfluv or Flat Bank & 109 & 44 & 0.2 \\
\hline Old Alluvial Fan & 1254 & 508 & 2.0 & Point Bar & 8 & 3 & $<0.1$ \\
\hline Alluvial Terrace & 75 & 30 & 0.1 & Levee & 204 & 82 & 0.3 \\
\hline Alluvial Plain Deposits & 5371 & 2174 & 8.7 & Flood Basin & 714 & 289 & 1.2 \\
\hline Meander Active Channel Deposits & 39 & 16 & 0.1 & Basins or Depressions & 1239 & 502 & 2.0 \\
\hline Meander Inactive Channel Deposits & 791 & 320 & 1.3 & Thermokarst Basin & 9 & 3 & $<0.1$ \\
\hline Meander Abandoned Channel Deposits & 248 & 101 & 0.4 & Waterbody & 653 & 264 & 1.1 \\
\hline Meander Active Overbank Deposits & 9 & 4 & $<0.1$ & Human Modified & 1502 & 608 & 2.4 \\
\hline Meander Inactive Overbank Deposits & 2 & 1 & $<0.1$ & & & & \\
\hline Meander Abandoned Overbank & 242 & 98 & 0.4 & & & & \\
\hline Active Tidal Flat & 1904 & 770 & 3.1 & Total & 61,996 & 25,089 & 100 \\
\hline Inactive Tidal Flat & 154 & 62 & 0.2 & & & & \\
\hline Fill and Embankments, undifferentiated & 1360 & 550 & 2.2 & & & & \\
\hline Excavations & 276 & 112 & 0.4 & & & & \\
\hline Deep Isolated Lakes, morainal & 272 & 110 & 0.4 & & & & \\
\hline Shallow Isolated Ponds, morainal & 54 & 22 & 0.1 & & & & \\
\hline Upper Perennial River, non-glacial & 20 & 8 & $<0.1$ & & & & \\
\hline Lower Perennial River, glacial & 56 & 23 & 0.1 & & & & \\
\hline Lower Perennial River, non-glacial & 34 & 14 & 0.1 & & & & \\
\hline Tidal Ponds & 122 & 50 & 0.2 & & & & \\
\hline Tidal River & 59 & 24 & 0.1 & & & & \\
\hline Nearshore Water & 26 & 11 & $<0.1$ & & & & \\
\hline Man-made Waterbodies & 9 & 3 & $<0.1$ & & & & \\
\hline Grand Total & 61,996 & 25,089 & 100 & & & & \\
\hline Total & 61,996 & 25,089 & 100 & & & & \\
\hline
\end{tabular}

commonly associated with the surface geomorphic unit are included in the descriptions in Table 4).

The dominant geomorphic units included Hillside Colluvium (22.5\% of mapped area) and Metamorphic Bedrock $(3.0 \%)$ in the mountains; Older Moraine (25.0\%), Glaciofluvial Channel (6.1\%), Kame (13.8\%), Alluvial Plain (8.7\%), and Bogs $(2.4 \%)$ in the glaci- ated lowlands; Meander Inactive Channel (1.3\%) on river floodplains; and Active Tidal Flats (3.1\%) along the coast (Table 4 ). Waterbodies were uncommon overall, but the dominant types were Deep Isolated Morainal Lakes $(0.4 \%)$, Tidal Ponds $(0.2 \%)$, and Lower Perennial Glacial River $(0.1 \%)$. This distribution of geomorphic units indicates that hillslope processes are domi- 


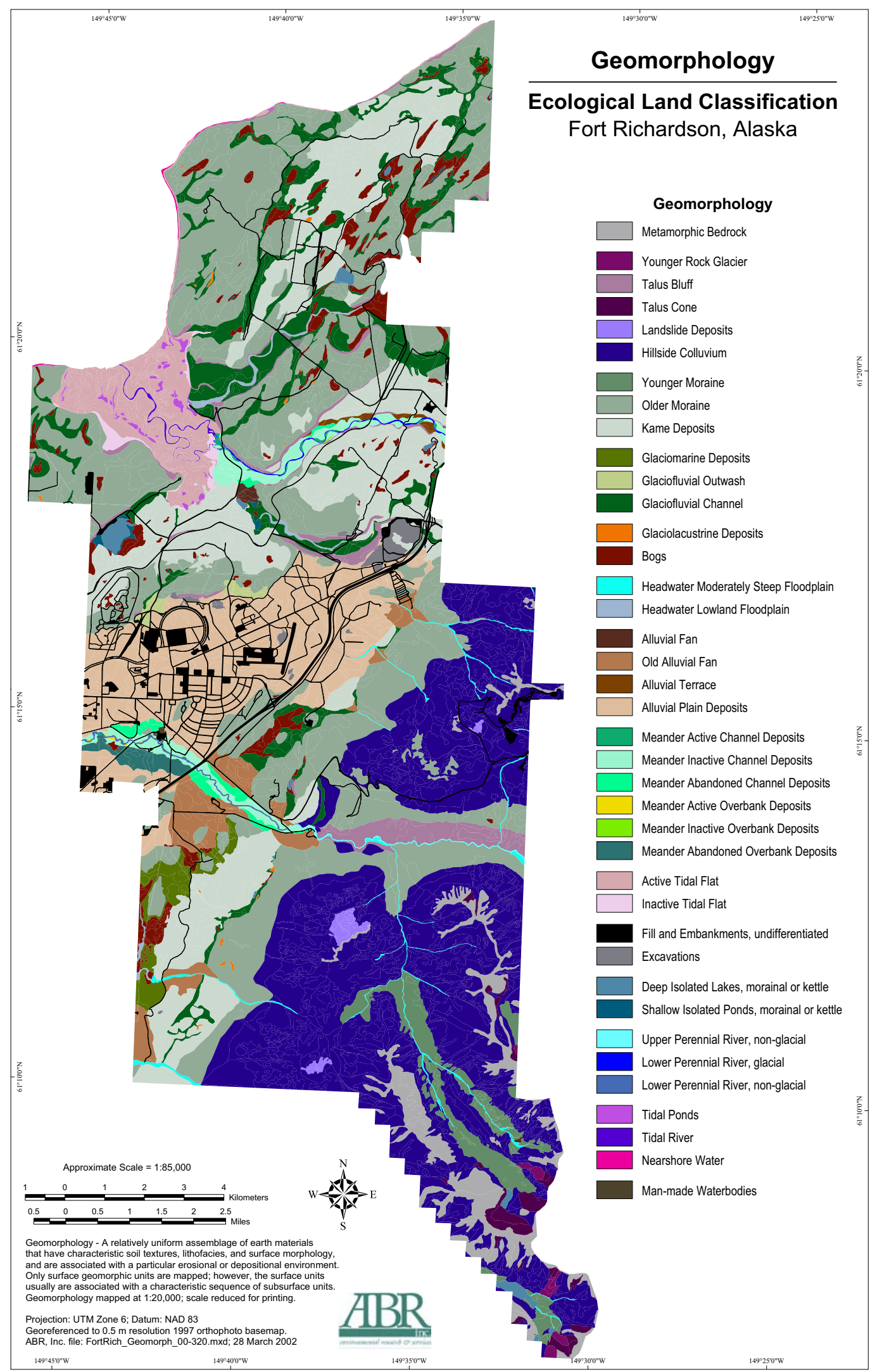

Figure 6. Map of geomorphic units on Fort Richardson, south-central Alaska, 2001. 
nant in the Chugach Mountains, while glacial, fluvial, and marine processes are dominant in the lowland portions of the study area.

Colluvial deposits accumulate on slopes primarily through the action of gravity, and to a lesser extent through the action of running water. For this ELS, we differentiated colluvium into several types: hillside colluvium, solifluction deposits, talus cones, talus bluffs, and landslide deposits. Because the mountain slopes were mostly glaciated during the late Pleistocene, the colluvial deposits are relatively young.

The glacial history of the study area is complex, and has affected most of the lowland portions of the base (Reger and Updike 1983, Reger et al. 1995, Hunter et al. 2000). Recent studies indicate that the glacial deposits are the result of multiple advances and retreats of the last glaciation. During the Late Wisconsin period, rapid changes occurred between glacial and marine environments with accompanying shifts in depositional regimes. Hunter et al. (2000) proposed eight glacial stages that left widespread glacial deposits in the area during the late Pleistocene. Stage 1, with full glacial conditions prior to $20,000 \mathrm{ybp}$, left lateral moraines near Rabbit Creek. Stage 7 was a major readvance $\sim 13,500-14,000{ }^{14} \mathrm{C}$ ybp and Stage 8, representing rapid retreat of ice, deposited the Elmendorf moraine.

Past and current fluvial processes also have strongly affected the landscape. During the last full glacial period, ice or moraine dams at the mouth of the Eagle River Valley periodically broke, causing rapid drainage of lakes impounded in the valley. Catastrophic flooding during these breakouts was deflected across the front of the Elmendorf Moraine and likely produced the broad sand and gravel alluvial plain (Mountain View fan) that extends across the Anchorage Glaciated Lowland to Knik Arm. Currently, the largest rivers in the study area are Eagle River and Ship Creek.

The geomorphic units are ecologically important because they represent areas with differing erosional and depositional environments, and therefore are affected by different natural disturbance regimes. For example, Meander Active Overbank Deposits are flooded frequently. The recurring sedimentation prevents development of a moss layer and contributes nutrients that presumably contribute to the vigorous growth of shrubs and saplings on the well-drained, circumneutral soils. Meander Abandoned Overbank Deposits, on the other hand, are rarely flooded and thus lack sediment input. Vegetation tends to be dominated by slow-growing, evergreen species that tolerate acidic, low-nutrient conditions.

The waterbody classification differentiates numerous characteristics that are ecologically important to invertebrates, fish, and wildlife. Glacial rivers are rich in sediment whereas nonglacial rivers have higher levels of humic and tannic compounds. Shallow ponds tend to melt earlier and become warmer than deep lakes and connected lakes allow better fish passage than isolated lakes. Riverine ponds are prone to flooding and sedimentation. Only a few of these characteristics were differentiated in the final ecotypes (see Ecotype section) to reduce the number of classes. For habitat studies, these waterbody types are preserved in the ITU code in the mapping and can be used for specific analyses.

Wherever possible we tried to be consistent with earlier mapping of surficial geology, but we made numerous modifications to facilitate our mapping of integrated terrain units. First, we created entirely new linework because the existing surficial geology maps (Yehle and Schmoll 1987, 1989; Yehle et al. 1990, Schmoll et al. 1996) compiled by Hunter et al. (2000) were not sufficiently co-registered with the black and white orthophoto mosaic. Second, we grouped many classes (Hunter et al. 2000) that had similar properties (e.g., we reduced the number of classes of glacial deposits). In this way, complexity was reduced from 191 surficial geology classes to 39 geomorphic units that were similar in concept to the engineering geology classification of ADGGS (1983). Third, we had slightly different concepts for some units, because we emphasized materials near the surface that are more ecologically relevant than the deeper deposits. Consequently, we differentiated active, inactive, and abandoned deposits on floodplains, restricted the use of the bedrock class to outcrop areas, expanded the use of hillside colluvium to include areas with thin $(>0.5 \mathrm{~m})$ layers of colluvium over bedrock, limited the differentiation of glaciofluvial channels to distinct, well-incised channels within moraines, grouped several intertidal zones into a single class (active tidal flat deposit), and differentiated numerous nearshore, river, and lake waterbody classes.

\section{Topography and surface forms}

Within Fort Richardson there is a large range in elevation, from sea level to $1615 \mathrm{~m}(5300 \mathrm{ft})$ at Tanaina Peak (Fig. 7). The topography can be divided into two broad areas that form the basis for physiographic districts, the low-lying glaciated areas around Anchorage and the highlands of the Chugach Mountains.

The area was classified into 24 surface forms on the basis of topographic characteristics (Fig. 8, Tables 4 and 5). The dominant surface form in the glaciated lowland was Moraine Complex (26.3\% of area), which occurs on kame and kettle topography associated with the melting of stagnant glacial ice. Flats $(21.4 \%)$ associated with fluvial processes also were abundant in the glaciated lowlands. Ten Upper Slope and Lower Slope classes were differentiated, primarily in the highland 


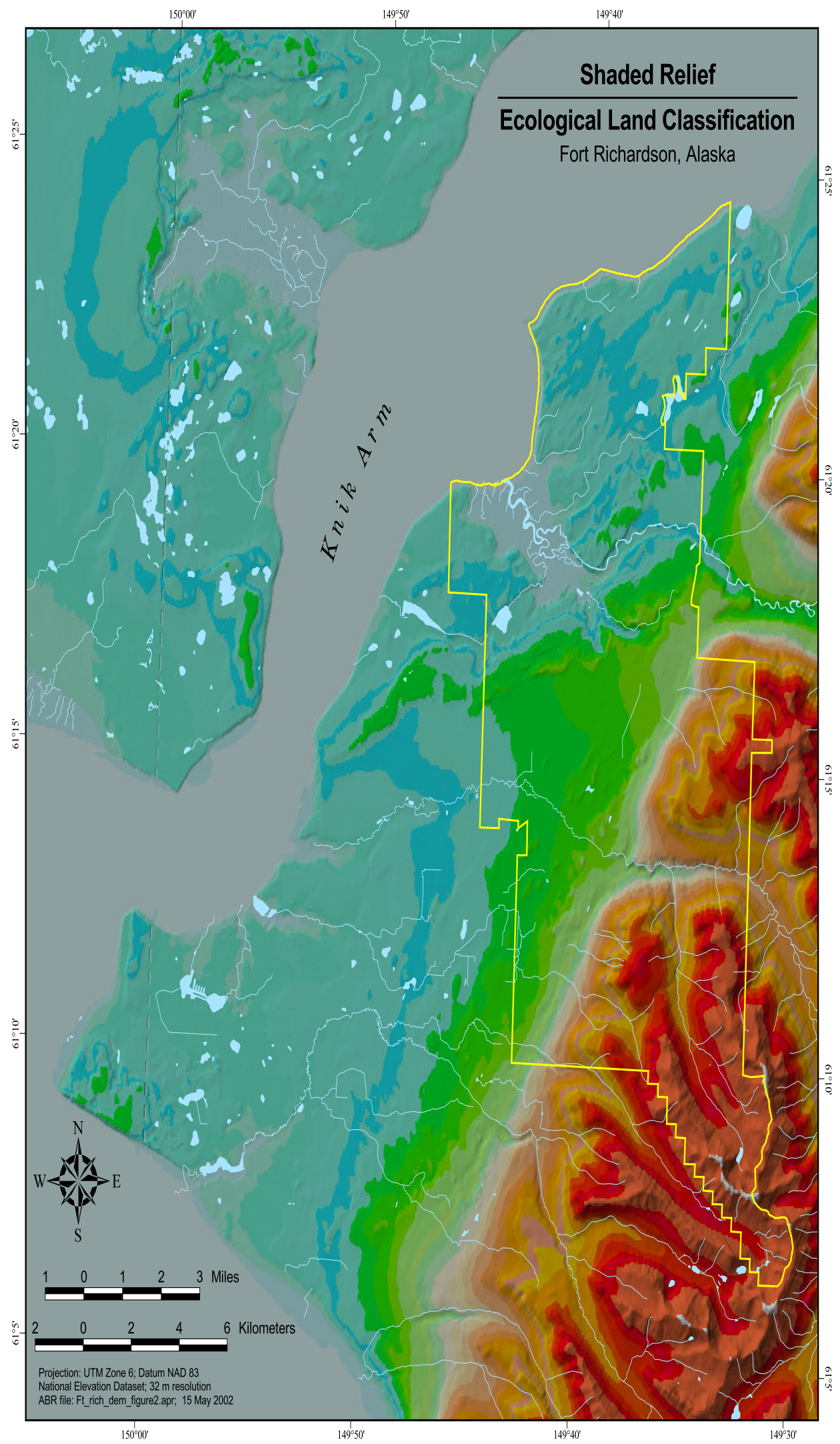

Figure 7. Map of topography illustrated as shaded relief for Fort Richardson, southcentral Alaska, 2001. 


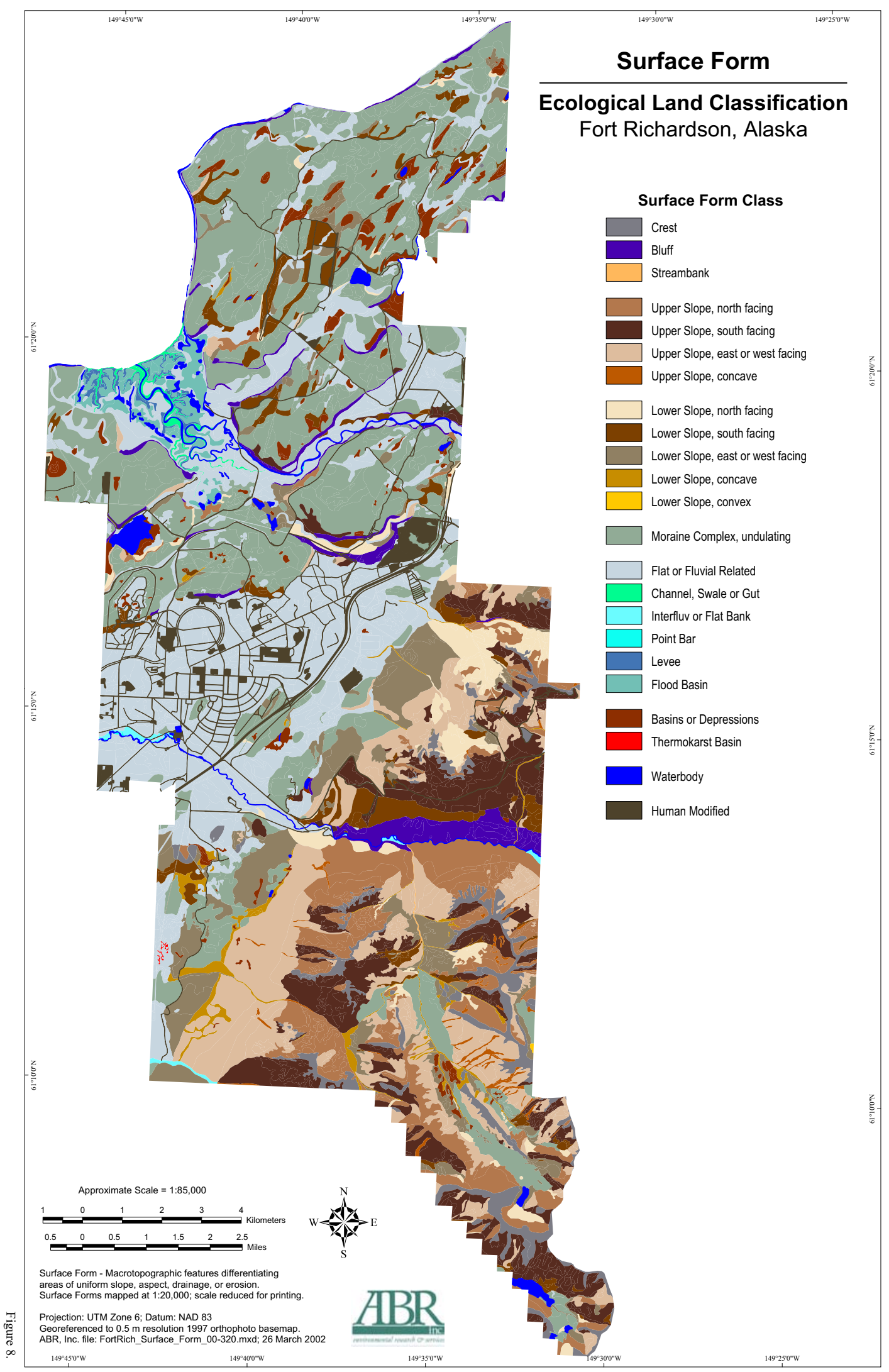

Figure 8. Map of surface forms on Fort Richardson, south-central Alaska, 2001. 
Table 5. Classification and descriptions of surface forms within Fort Richardson, southcentral Alaska, 2001.

\begin{tabular}{|c|c|}
\hline Class & Description \\
\hline Crest (C) & $\begin{array}{l}\text { The windswept upper-most portion of a hill, usually convex in all directions with no distinct } \\
\text { aspect. }\end{array}$ \\
\hline Bluff (Sb) & Very steep slope that typically occurs along a river where the slope is undercut by the river. \\
\hline Streambanks (St) & Relatively steep slopes formed by small headwater river and stream channels. \\
\hline $\begin{array}{l}\text { Upper Slope, North } \\
\text { Facing (Sun) }\end{array}$ & $\begin{array}{l}\text { Generally convex upper and middle portions of a hill or slope on north-facing }\left(315-45_{i}\right) \\
\text { slopes. The surface typically is erosional, water shedding, and receives less solar radiation. }\end{array}$ \\
\hline $\begin{array}{l}\text { Upper Slope, South } \\
\text { Facing (Sus) }\end{array}$ & $\begin{array}{l}\text { Generally convex upper and middle portions of a hill or slope on south-facing }\left(135-225_{i}\right) \\
\text { slopes. The surface typically is erosional, water shedding, and exposed to more solar radiation. }\end{array}$ \\
\hline $\begin{array}{l}\text { Upper Slope, East Or } \\
\text { West Facing (Sue) }\end{array}$ & $\begin{array}{l}\text { Generally convex upper and middle portions of a hill or slope below the shoulder on east } \\
\left(45-135_{i}\right) \text { and west }\left(225-315_{i}\right) \text { facing slopes. The surface typically is erosional and water } \\
\text { shedding. }\end{array}$ \\
\hline $\begin{array}{l}\text { Upper Slope, Concave } \\
\text { (Suc) }\end{array}$ & $\begin{array}{l}\text { Concave upper and middle portions of a hill or slope. Not aspect specific. The surface typically } \\
\text { is depositional and water collecting, }\end{array}$ \\
\hline Lower Slope (S1) & Lower portion of a hill or slope, not aspect or shape specific. \\
\hline $\begin{array}{l}\text { Lower Slope, North } \\
\text { Facing (Sln) }\end{array}$ & $\begin{array}{l}\text { Generally concave lower portion of a hill or slope on north-facing }(315-45 i) \text { slopes. The surface } \\
\text { typically is depositional, water collecting, and receives less solar radiation. }\end{array}$ \\
\hline $\begin{array}{l}\text { Lower Slope, South } \\
\text { Facing (Sls) }\end{array}$ & $\begin{array}{l}\text { Generally concave lower portion of a hill or slope on south-facing }\left(135-225_{i}\right) \text { slopes. The } \\
\text { surface typically is depositional and water collecting. }\end{array}$ \\
\hline $\begin{array}{l}\text { Lower Slope, East Or } \\
\text { West Facing (Sle) }\end{array}$ & $\begin{array}{l}\text { Generally concave lower portion of a hill or slope on east }\left(45-135_{i}\right) \text { and west }\left(225-315_{i}\right) \text { facir } \\
\text { slopes. The surface typically is depositional and water collecting. }\end{array}$ \\
\hline $\begin{array}{l}\text { Lower Slope, Concave } \\
\text { (Slc) }\end{array}$ & $\begin{array}{l}\text { Generally concave lower portion of a hill or slope when aspect is not differentiated. The surface } \\
\text { typically is depositional and water collecting. }\end{array}$ \\
\hline $\begin{array}{l}\text { Lower Slope, Convex } \\
\text { (Slv) }\end{array}$ & $\begin{array}{l}\text { Convex lower portion of a hill or slope. Usually erosional and water shedding. Not aspect } \\
\text { specific. }\end{array}$ \\
\hline $\begin{array}{l}\text { Moraine Complex, } \\
\text { Undulating }(\mathrm{Xm})\end{array}$ & $\begin{array}{l}\text { Undulating surfaces with ridges and swales (depressions) associated with glacial deposits. The } \\
\text { highly interspersed slopes are too small to be mapped separately. }\end{array}$ \\
\hline $\begin{array}{l}\text { Flat or Fluvial Related } \\
\text { (F) }\end{array}$ & $\begin{array}{l}\text { Level areas, usually originated as a result of water flow within or beyond a river channel. } \\
\text { Included in this class are terraces, nonpatterned flat areas, and flat bars. }\end{array}$ \\
\hline $\begin{array}{l}\text { Channel, Swale or Gut } \\
\text { (Fc) }\end{array}$ & $\begin{array}{l}\text { Low-lying concave portions of the floodplain developed from river scouring. Tend to be water } \\
\text { gathering. }\end{array}$ \\
\hline Interfluv (Fi) & Flat areas on floodplains that are slightly raised above adjacent lower active or paleo-channels. \\
\hline Point Bar (Fbp) & $\begin{array}{l}\text { Flat to gently sloping, crescent shaped shoal forming immediately adjacent to a river, usually } \\
\text { forming on the inside of a bend. }\end{array}$ \\
\hline Levee $(\mathrm{Fl})$ & $\begin{array}{l}\text { Flat, prominent to indistinct raised surface on a floodplain or tidal flat immediately adjacent to } \\
\text { a channel or waterbody. }\end{array}$ \\
\hline Flood Basin (Ff) & Flat, distal portion of a floodplain behind a levee. Surface tends to impound water. \\
\hline $\begin{array}{l}\text { Basins or Depressions } \\
\text { (B) }\end{array}$ & $\begin{array}{l}\text { An area that is concave in all directions. Often collects water. This class includes kettle holes, } \\
\text { formed by the melting of a glacial ice mass formed on the surface of glacial drift. }\end{array}$ \\
\hline Thermokarst (Bt) & Collapse-scar depressions formed by thawing of permafrost. \\
\hline Waterbody (W) & Fresh or marine water. \\
\hline Human Modified (Hm) & Complex surfaces that have been modified by human activity. \\
\hline
\end{tabular}

area in the Chugach Mountains where areas of uniform slope position and aspect were large enough to map separately.

Elevation gradients are ecologically important because they control water movement, provide the energy for mass wasting of rocks, and distribution of surficial materials. The importance of slope is evident in the distribution of geomorphic units (Fig. 6). Exposed Metamorphic Bedrock and Talus Cones occur on the highest, steepest slopes where potential energy is highest and erosional processes predominate. Hillside Colluvium occurs on intermediate slopes where both erosion and deposition are active. Alluvial Plain, Older Moraine, and Kame Deposits are found mostly in the lowland areas, where potential energy is low and depositional processes predominate. The elevation gradient also affects air temperatures and ecological responses. As a result of the strong decrease in temperature with elevation, vegetation structure in the alpine zone is limited to dwarf shrubs and herbaceous plants.

\section{Vegetation}

Vegetation in the study area was classified using Level IV of the Alaska Vegetation Classification (AVC) 
system (Viereck et al. 1992) and by developing plant associations (Level V) based on quantitative analysis of floristic composition. The plant associations had the advantage of being closely related to individual ecotypes (see Ecotypes), but they were difficult to recognize on aerial photography. The AVC, however, performs well at differentiating structural characteristics visible on aerial photos and is a fairly robust, well-established classification system. When combined with geomorphic and surface form characteristics during mapping, AVC Level IV classes also provided a strong basis for differentiating plant associations (AVC Level V) and ecotypes.

We differentiated 46 vegetated classes based on Level IV of the AVC, as well as five nonvegetated (e.g., Barrens, Brackish Water) classes (Fig. 9, Tables 6 and 7). Nonvegetated classes are not included in the AVC. The dominant vegetation class was Open Spruce-Paper Birch Forest (26.2\% of area). Other common forest classes included Closed Paper Birch (8.1\%), Open Paper Birch (5.7\%), and Open Black Spruce-White Spruce (2.3\%). Common shrub classes included Closed Tall Alder (3.5\%), Open Tall Alder (3.0\%), Open Low Shrub Birch-Willow (2.5\%), Crowberry Tundra (5.1\%), and Dryas-Lichen Tundra (8.0\%). The only common herbaceous vegetation types were halophytic meadows in the coastal zone.

\section{Ecosystems and landscapes}

\section{Hierarchical organization of ecological} components

Toposequences The ecosystem classification was based primarily on the survey of ecosystem components (e.g., topography, geomorphology, soil, hydrology, permafrost, and vegetation) along the toposequences. Representative cross-sectional profiles were constructed to illustrate relationships among ecosystem components on six of the 16 toposequences (Fig. 10-15). The toposequences display two-dimensional views of the lithofacies that were used as the basis for classifying and mapping geomorphic units. Examples from various ecosubdistricts within three of the four ecodistricts (see Ecodistricts) in the study area are described below, to illustrate some of the main ecological relationships. Vegetation classes follow the AVC.

Within the Eagle River Flats along the Upper Cook Inlet Coast, geomorphology was dominated by Active and Inactive Tidal Flats, and Nearshore Water (Fig. 10). The topography was flat with only slight variation due to formation of tidal guts and levees. The soils on the Active Tidal Flats were loamy, poorly drained, and lacked organic matter accumulation. The soils on Inactive Tidal Flats were similar but had moderately thick organic accumulations. Vegetation on the Active Tidal
Flats was salt-tolerant and included Halophytic Herb Wet Meadow, Halophytic Grass Wet Meadow, Halophytic Sedge Wet Meadow (dominated by Carex ramenskii), Halophytic Sedge Marsh, and Elymus Meadow. On Inactive Tidal Flats where the water was slightly brackish, Halophytic Sedge Wet Meadow (dominated by C. lyngbyaei) and Sweetgale-Graminoid Shrub Meadow were common. Ecological relationships for the Eagle River flats were based on Racine et al. (1993).

Within the Eagle River floodplain (transect 1), the geomorphology was dominated by Meander Floodplain Active Channel Deposit, Meander Floodplain Inactive Channel Deposit, and Meander Floodplain Abandoned Channel Deposit (Fig. 11). The toposequence extended onto Kame and Drumlin Deposits. The excessively drained, gravelly soils of the active channel were Partially Vegetated with Populus balsamifera trichocarpa, Alnus tenuifolia, and Epilobium spp. The higher, Meander Floodplain Inactive Channel Deposits had welldrained sandy to gravelly soils with interbedded organics that supported Open Black Cottonwood Forest and Open Black Cottonwood-White Spruce Forest. Meander Abandoned Channel Deposits with well-drained gravelly soils had Open White Spruce Forest, while lower poorly drained areas had Open Black SpruceWhite Spruce Forest. Inactive channels with thick accumulations of silts and organics supported Open Tall Alder swamps.

Within the Anchorage Glaciated Lowlands near the cantonment, the geomorphology was dominated by old Alluvial Plain deposits with well-drained soils (Fig. 12). Adjacent to this flat terrain were undulating Older Moraine deposits with Bogs in the depressions. Vegetation on the Alluvial Plain was Open Quaking Aspen-Spruce Forest and Open Spruce-Paper Birch Forests that were dominated by plants typical of the boreal forest. The Alluvial Plain deposits had rocky soils, welldrained soil, with thin organic horizons. Low-lying Bogs had Open Black Spruce Forest and Lowland Loamy Moist Meadow vegetation.

Within the Knik Glaciated Lowlands, the geomorphology included complex interspersions of Older Moraine, Drumlins, Glaciofluvial Channel, Bog, and Lacustrine deposits (Fig. 13). The Older Moraine and Drumlin deposits had well-drained, rocky soils that typically supported Closed Paper Birch Forest and Open Spruce-Paper Birch Forest on upper slopes, but occasionally had Closed Quaking Aspen Forest on steep, south-facing slopes. Low-lying Glaciofluvial Channel deposits had somewhat poorly drained soils that were dominated by Open Black Spruce Forest and Open Black Spruce-White Spruce Forest. Depressions with 


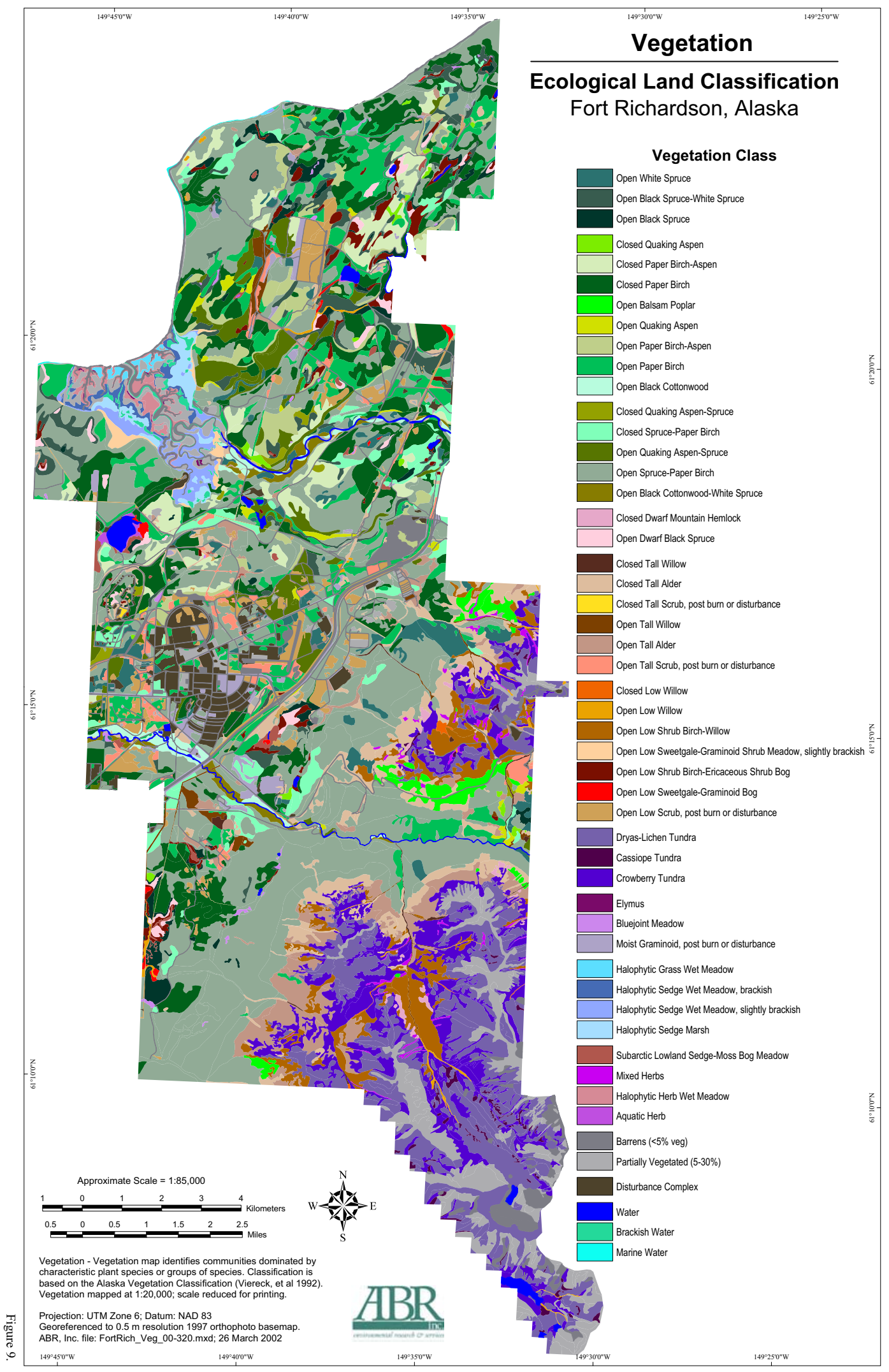

Figure 9. Map of vegetation classes on Fort Richardson, south-central Alaska, 2001. 
Table 6. Classification and description of vegetation within Fort Richardson, south-central Alaska, 2001. Classification and descriptions based on the Alaska Vegetation Classification (Viereck et al. 1992).

\begin{tabular}{|c|c|}
\hline Class & Description \\
\hline $\begin{array}{l}\text { Open White Spruce } \\
\text { (Fnows) }\end{array}$ & $\begin{array}{l}\text { Open needleleaf forests with } 25-60 \% \text { tree cover which are dominated by Picea glauca. This } \\
\text { type is found on uplands, lowlands, and floodplains. Other trees (Betula papyrifera, Picea } \\
\text { mariana) may be present but they are not co-dominant. Understory species include Cornus } \\
\text { canadensis, Empetrum nigrum, Linnaea borealis, Rosa acicularis, Calamagrostis } \\
\text { canadensis, and Vaccinium vitis-idaea. Feather mosses form a continuous carpet. Lowland } \\
\text { forests are distinguished by the presence of Ledum groenlandicum, and riverine by the } \\
\text { presence of Alnus tenuifolia. }\end{array}$ \\
\hline $\begin{array}{l}\text { Open Black } \\
\text { Spruce-White Spruce } \\
\text { (Fnobw) }\end{array}$ & $\begin{array}{l}\text { Open ( } 25-60 \% \text { tree cover) needleleaf forests on floodplains and lowlands in which Picea } \\
\text { eglauca and Picea mariana are co-dominants. Shrubs dominate the understory, including } \\
\text { Ledum groenlandicum, Empetrum nigrum, Rosa acicularis, Linnaea borealis, and } \\
\text { Vaccinium vitis-idaea. Cornus canadensis, Calamagrostis canadensis, Equisetum arvense, } \\
\text { and feather mosses also are common. Riverine forests are distinguished by the presence of } \\
\text { Alnus tenuifolia. }\end{array}$ \\
\hline $\begin{array}{l}\text { Open Black Spruce } \\
\text { (Fnobs) }\end{array}$ & $\begin{array}{l}\text { Open Picea mariana lowland forests and forest bogs with } 25-60 \% \text { tree cover. Associated } \\
\text { species include Ledum groenlandicum, Empetrum nigrum, Vaccinium vitis-idaea, Cornus } \\
\text { canadensis, V. uliginosum, Rubus chamaemorus, and Equisetum silvaticum. Feather mosses } \\
\text { are common in lowland forests and Sphagnum spp., in bogs. }\end{array}$ \\
\hline $\begin{array}{l}\text { Closed Quaking } \\
\text { Aspen (Fbca) }\end{array}$ & $\begin{array}{l}\text { Dry upland forests with over } 60 \% \text { cover of Populus tremuloides. The understory is sparse } \\
\text { with Picea glauca, Rosa acicularis, Ledum groenlandicum, Hylocomium splendens, } \\
\text { Pleurozium schreberi, and Ptilium crista-castrensis. }\end{array}$ \\
\hline $\begin{array}{l}\text { Closed Paper } \\
\text { Birch-Aspen (Fbcba) }\end{array}$ & $\begin{array}{l}\text { Upland and lowland moist forests with over } 60 \% \text { cover and tree canopy co-dominated by } \\
\text { Betula papyrifera and Populus tremuloides. The understory is similar to Closed Paper Birch } \\
\text { Forest. }\end{array}$ \\
\hline $\begin{array}{l}\text { Closed Paper Birch } \\
\text { (Fbcb) }\end{array}$ & $\begin{array}{l}\text { Closed ( }>60 \% \text { tree cover) upland and lowland forests dominated by Betula papyrifera. Picea } \\
\text { glauca is common in the understory. Other associated species include Viburnum edule, } \\
\text { Ledum groenlandicum (lowlands), Vaccinium vitis-idaea, Gymnocarpium dryopteris, } \\
\text { Cornus canadensis, Calamagrostis canadensis, Lycopodium annotinum, Linnaea borealis, } \\
\text { and Pleurozium schreberi. }\end{array}$ \\
\hline $\begin{array}{l}\text { Open Balsam Poplar } \\
\text { (Fbop) }\end{array}$ & $\begin{array}{l}\text { Open ( } 25-60 \% \text { tree cover) subalpine forests dominated by Populus balsamifera balsamifera } \\
\text { Trees may be severely dwarfed. Associated species include Viburnum edule, Epilobium } \\
\text { angustifolium, Gymnocarpium dryopteris, Cornus canadensis, Sanguisorba stipulata, } \\
\text { Geranium erianthum, Heracleum lanatum, and Aconitum delphinifolium. }\end{array}$ \\
\hline $\begin{array}{l}\text { Open Quaking Aspen } \\
\text { (Fboa) }\end{array}$ & $\begin{array}{l}\text { Open, upland and lowland forests dominated by } 25-60 \% \text { cover of Populus tremuloides. } \\
\text { Soils may be dry or moist. Understory species include Picea glauca, Viburnum edule, } \\
\text { Shepherdia canadensis (uplands), Ledum groenlandicum (lowlands), Calamagrostis } \\
\text { canadensis, Linnaea borealis, Pyrola asarifolia, Rosa acicularis, and Vaccinium vitis- } \\
\text { idaea. }\end{array}$ \\
\hline $\begin{array}{l}\text { Open Paper } \\
\text { Birch-Aspen (Fboba) }\end{array}$ & $\begin{array}{l}\text { Open ( } 25-60 \% \text { tree cover) upland and lowland forests, co-dominated by Betula papyrifera } \\
\text { and Populus tremuloides. The understory is similar to Open Paper Birch Forest. }\end{array}$ \\
\hline $\begin{array}{l}\text { Open Paper Birch } \\
\text { (Fbob) }\end{array}$ & $\begin{array}{l}\text { Upland and lowland forests with an open }(25-60 \%) \text { cover of Betula papyrifera. Picea glauc } \\
\text { may be common in the understory. Associated species include Alnus sinuata, Viburnum } \\
\text { edule, Vaccinium vitis-idaea, Gymnocarpium dryopteris, Cornus canadensis, Calamagrostis } \\
\text { canadensis, Equisetum arvense, and Linnaea borealis. }\end{array}$ \\
\hline $\begin{array}{l}\text { Open Black } \\
\text { Cottonwood (Fboc) }\end{array}$ & $\begin{array}{l}\text { Open ( } 25-60 \% \text { tree cover) riverine forests dominated by Populus balsamifera trichocarpa. } \\
\text { Picea glauca may be common in the understory. Viburnum edule, Alnus tenuifolia, Rosa } \\
\text { acicularis, Athyrium filix-femina, Heracleum lanatum, Calamagrostis canadensis, } \\
\text { Equisetum arvense, and Trientalis europaea are common in the understory. }\end{array}$ \\
\hline
\end{tabular}


Table 6. (cont'd).

\begin{tabular}{|c|c|}
\hline Class & Description \\
\hline $\begin{array}{l}\text { Closed Quaking } \\
\text { Aspen-Spruce } \\
\text { (Fmcas) }\end{array}$ & $\begin{array}{l}\text { Moist upland or lowland closed ( }>60 \% \text { tree cover) forests co-dominated Populus tremuloide } \\
\text { and Picea glauca. Associated species are similar to Open Quaking Aspen-Spruce Forest. }\end{array}$ \\
\hline $\begin{array}{l}\text { Closed Spruce-Pape } \\
\text { Birch (Fmcsb) }\end{array}$ & $\begin{array}{l}\text { Closed ( }>60 \% \text { tree cover) upland or lowland forests dominated by Betula papyrifera and } \\
\text { Picea glauca; Salix bebbiana, Cornus canadensis, Vaccinium vitis-idaea, Hylocomium } \\
\text { splendens, and Pleurozium schreberi are associated species. Alnus sinuata distinguished } \\
\text { upland forests. }\end{array}$ \\
\hline $\begin{array}{l}\text { Open Quaking } \\
\text { Aspen-Spruce } \\
\text { (Fmoas) }\end{array}$ & $\begin{array}{l}\text { Upland or lowland forests with an open canopy ( } 25-60 \% \text { cover) co-dominated by Populus } \\
\text { tremuloides and Picea glauca. Common understory species include Rosa acicularis, } \\
\text { Viburnum edule, Ledum groenlandicum, Epilobium angustifolium, Cornus canadensis, } \\
\text { Festuca altaica, Linnaea borealis, Vaccinium vitis-idaea, and feather mosses. Shepherdia } \\
\text { canadensis distinguishes upland forests. }\end{array}$ \\
\hline $\begin{array}{l}\text { Open Spruce-Paper } \\
\text { Birch (Fmosb) }\end{array}$ & $\begin{array}{l}\text { Upland, lowland, or riverine forests with an open ( } 25-60 \% \text { cover) canopy co-dominated by } \\
\text { Betula papyrifera and Picea glauca. Understory species include Viburnum edule, } \\
\text { Gymnocarpium dryopteris, Cornus canadensis, Epilobium angustifolium, Rosa acicularis, } \\
\text { Vaccinium vitis-idaea, Linnaea borealis, and Calamagrostis canadensis. Alnus sinuata and } \\
\text { Ribes triste distinguish upland forests and Populus balsamifera trichocarpa distinguishes } \\
\text { floodplain forests. }\end{array}$ \\
\hline $\begin{array}{l}\text { Open Black } \\
\text { Cottonwood-White } \\
\text { Spruce (Fmocws) }\end{array}$ & $\begin{array}{l}\text { Riverine forests with } 25-60 \% \text { tree cover co-dominated by Populus balsamifera trichocarpa } \\
\text { and Picea glauca. Associated species include Alnus tenuifolia, Calamagrostis canadensis, } \\
\text { Galium triflorum, Linnaea borealis, Mertensia paniculata, Trientalis europaea, Artemisia } \\
\text { tilesii, and Rosa acicularis. }\end{array}$ \\
\hline $\begin{array}{l}\text { Closed Dwarf } \\
\text { Mountain Hemlock } \\
\text { (Sfcmh) }\end{array}$ & $\begin{array}{l}\text { Subalpine, dwarf forests with a closed }(<60 \% \text { cover) canopy of Tsuga mertensiana. } \\
\text { Associated plants include Cornus canadensis, Empetrum nigrum, Lycopodium annotinum, } \\
\text { Gymnocarpium dryopteris, Linnaea borealis, Rubus pedatus, Calamagrostis canadensis, } \\
\text { and Pleurozium schreberi. }\end{array}$ \\
\hline $\begin{array}{l}\text { Open Dwarf Black } \\
\text { Spruce (Sfobs) }\end{array}$ & $\begin{array}{l}\text { Open forests }(25-60 \% \text { cover }) \text { of Picea mariana in which most trees are }<3 \mathrm{~m} \text { tall. These } \\
\text { forests commonly are found on bogs in association with Rubus chamaemorus, Ledum } \\
\text { groenlandicum, L. palustre decumbens, Vaccinium uliginosum, V. vitis-idaea, Empetrum } \\
\text { nigrum, Oxycoccus microcarpus, and Sphagnum spp. }\end{array}$ \\
\hline $\begin{array}{l}\text { Closed Tall Willow } \\
\text { (Stcw) }\end{array}$ & $\begin{array}{l}\text { Willow thickets, common along streambeds and in the subalpine where shrub cover is at } \\
\text { least } 75 \% \text { and over } 1.5 \mathrm{~m} \text { tall. Salix alaxensis is dominant along streams while } S . \text { barclayi } \\
\text { and } S . \text { scouleriana commonly are dominant in the subalpine. Associated species include } \\
\text { Gymnocarpium dryopteris, Alnus sinuata, S. planifolia pulchra, Heracleum lanatum, and } \\
\text { Mertensia paniculata. }\end{array}$ \\
\hline $\begin{array}{l}\text { Closed Tall Alder } \\
\text { (Stca) }\end{array}$ & $\begin{array}{l}\text { This class occurs in all physiographic types except alpine and coastal. Closed stands of over } \\
75 \% \text { cover of Alnus canaden in upland, lowland and subalpine sites and A. tenuifolia on } \\
\text { floodplains. Associated species include Thalictrum sparsiflorum, Calamagrostis anadensis } \\
\text { Dryopteris dilitata, Equisetum arvense, Trientalis europaea, Epilobium angustifolium, and } \\
\text { Oplopanax horridus. }\end{array}$ \\
\hline $\begin{array}{l}\text { Closed Tall Scrub, } \\
\text { post burn or } \\
\text { disturbance (Stcd) }\end{array}$ & $\begin{array}{l}\text { Closed stands of over } 75 \% \text { tall shrubs and tree saplings that have been disturbed by human } \\
\text { activity. Species composition is variable but can include Alnus canaden, Salix scouleriana, } \\
\text { S. bebbiana, Betula papyrifera, Populus tremuloides, and P. balsamifera trichocarpa. }\end{array}$ \\
\hline $\begin{array}{l}\text { Open Tall Willow } \\
\text { (Stow) }\end{array}$ & $\begin{array}{l}\text { Open stands of tall shrub (>1.5m tall) in the subalpine and on streambanks dominated by } \\
\text { Salix scouleriana or Salix alaxensis, respectively. Associated species include Sanguisorba } \\
\text { stipulata, Geranium erianthum, Epilobium angustifolium, Calamagrostis canadensis, } \\
\text { Betula nana, Aconitum delphinifolium, Heracleum lanatum, and Equisetum arvense. }\end{array}$ \\
\hline
\end{tabular}


Table 6 (cont'd). Classification and description of vegetation within Fort Richardson, southcentral Alaska, 2001. Classification and descriptions based on the Alaska Vegetation Classification (Viereck et al. 1992).

\begin{tabular}{|c|c|}
\hline Class & Description \\
\hline $\begin{array}{l}\text { Open Tall Alder } \\
\text { (Stoa) }\end{array}$ & $\begin{array}{l}\text { Riverine, lowland, and upland stands of open ( } 25-75 \% \text { shrub cover) tall shrub }(>1.5 \mathrm{~m} \text { tal: } \\
\text { dominated by Alnus sinuata or Alnus tenuifolia (riverine). Calamagrostis canadensis, } \\
\text { Equisetum arvense, Thalictrum sparsiflorum, Polemonium acutiflorum and Galium } \\
\text { triflorum are common. }\end{array}$ \\
\hline $\begin{array}{l}\text { Open Tall Scrub, post } \\
\text { burn or disturbance } \\
\text { (Stod) }\end{array}$ & $\begin{array}{l}\text { Sites where vegetation has been altered by human activity and now support open stands of } \\
<75 \% \text { shrub cover, }>1.5 \mathrm{~m} \text { tall. Species composition is variable and similar to Closed } \\
\text { Tall Scrub, post burn or disturbance. }\end{array}$ \\
\hline $\begin{array}{l}\text { Closed Low Willow } \\
\text { (Slcw) }\end{array}$ & $\begin{array}{l}\text { Low }(0.2-1.5 \mathrm{~m}) \text { shrub stands on streambanks of }>75 \% \text { cover usually dominated by Salix } \\
\text { planifolia pulchra. Understory species may include Sanguisorba stipulata and } \\
\text { Gymnocarpium dryopteris }\end{array}$ \\
\hline $\begin{array}{l}\text { Open Low Willow } \\
\text { (Slow) }\end{array}$ & $\begin{array}{l}\text { Alpine low }(0.2-1.5 \mathrm{~m}) \text { shrub stands of } 25-75 \% \text { cover commonly dominated by Salix } \\
\text { barclayi and/or } S \text {. pulchra. Understory species may include Calamagrostis canadensis, } \\
\text { Epilobium angustifolium, Empetrum nigrum, Luzula parviflora, and Rubus arcticus. }\end{array}$ \\
\hline $\begin{array}{l}\text { Open Low Shrub } \\
\text { Birch-Willow } \\
\text { (Slobw) }\end{array}$ & $\begin{array}{l}\text { Open subalpine shrub communities of } 25-75 \% \text { cover }(0.2-1.5 \mathrm{~m} \text { tall), dominated by } \\
\text { Betula nana and Salix scouleriana. Empetrum nigrum, and Pleurozium schreberi are } \\
\text { common dominants with B. occidentalis, Rosa acicularis, Festuca altaica, Epilobium } \\
\text { angustifolium, Aconitum delphinifolium, Vaccinium uliginosum, Cornus canadensis, and } \\
\text { Geranium erianthum. }\end{array}$ \\
\hline $\begin{array}{l}\text { Open Low } \\
\text { Sweetgale-Graminoid } \\
\text { Shrub Meadow, } \\
\text { slightly brackish } \\
\text { (Slomg) }\end{array}$ & $\begin{array}{l}\text { A coastal community with } 25-75 \% \text { of low shrub cover }(0.2-1.5 \mathrm{~m} \text { tall }) \text { dominated by } \\
\text { Myrica gale. Carex lyngbyaei, Calamagrostis sp., Salix ovalifolia, and Comarum palustre } \\
\text { also are common in this class. }\end{array}$ \\
\hline $\begin{array}{l}\text { Open Low Shrub } \\
\text { Birch-Ericaceous } \\
\text { Shrub Bog (Slobb) }\end{array}$ & $\begin{array}{l}\text { Open bog communities of } 25-75 \% \text { low shrub }(0.2-1.5 \mathrm{~m} \text { tall }) \text { cover, dominated by Betula } \\
\text { nana with Rubus chamaemorus, Sphagnum spp. Picea mariana, Ledum palustre } \\
\text { decumbens, and Oxycoccus microcarpus. }\end{array}$ \\
\hline $\begin{array}{l}\text { Open Low } \\
\text { Sweetgale-Graminoid } \\
\text { Bog (Slocg) }\end{array}$ & $\begin{array}{l}\text { Bogs and poor fens with } 25-75 \% \text { low shrub cover }(0.2-1.5 \mathrm{~m} \text { tall) dominated by Myrica } \\
\text { gale and Calamagrostis canadensis. Associated species include Comarum palustre, Betula } \\
\text { nana, Carex aquatilis, and Sphagnum spp. }\end{array}$ \\
\hline $\begin{array}{l}\text { Open Low Scrub, } \\
\text { post burn or } \\
\text { disturbance (Slod) }\end{array}$ & $\begin{array}{l}\text { Sites affected by human activity that currently support low shrub cover of } 25-75 \% \text {, most } \\
\text { of which is }<1.5 \mathrm{~m} \text { tall. Low shrubs include Salix bebbiana, S. scouleriana, Ledum } \\
\text { groenlandicum, Rosa acicularis and Viburnum edule. Tree saplings and Cornus } \\
\text { canadensis, Calamagrostis canadensis, Empetrum nigrum, Lupinus nootkatensis and } \\
\text { Lycopodium spp. may be present. }\end{array}$ \\
\hline $\begin{array}{l}\text { Dryas-Lichen Tundra } \\
\text { (Sddl) }\end{array}$ & $\begin{array}{l}\text { Alpine tundra dominated by Dryas octopetala and lichens (Flavocetraria, Bryocaulon, } \\
\text { Cladina and Cladonia species). Hierochlo alpina, Salix arctica, Empetrum nigrum, } \\
\text { Diapensia lapponica, Vaccinium vitis-idaea, Oxytropis bryophila, and Carex michrochaeta } \\
\text { are common. }\end{array}$ \\
\hline $\begin{array}{l}\text { Cassiope Tundra } \\
\text { (Sdec) }\end{array}$ & $\begin{array}{l}\text { Alpine tundra, commonly in snowbed hollows or concave slopes, dominated by the dwarf } \\
\text { shrub Cassiope stelleriana with Luetkea pectinata. Associated plants include Empetrum } \\
\text { nigrum, Lycopodium alpinum, Huperzia selago, Gentiana glauca, and Cladina stellaris. }\end{array}$ \\
\hline $\begin{array}{l}\text { Crowberry Tundra } \\
\text { (Sdee) }\end{array}$ & $\begin{array}{l}\text { Moist alpine tundra dominated by Empetrum nigrum. Other common species include } \\
\text { Betula nana, Vaccinium uliginosum, V. vitis-idaea, Arctous alpina, and Cornus } \\
\text { canadensis }\end{array}$ \\
\hline Elymus (Hgdl) & $\begin{array}{l}\text { Coastal sandy communities dominated by the species Leymus mollis (Elymus arenarius } \\
\text { mollis). Other species include Potentilla egedii, Plantago maritima, and Triglochin } \\
\text { maritimum. }\end{array}$ \\
\hline
\end{tabular}


Table 6. (cont'd).

\begin{tabular}{|c|c|}
\hline Class & Description \\
\hline $\begin{array}{l}\text { Bluejoint Meadow } \\
(\text { Hgmb) }\end{array}$ & $\begin{array}{l}\text { Moist meadows in uplands or lowlands dominated by Calamagrostis canadensis. Epilobium } \\
\text { angustifolium, Mertensia paniculata, Betula papyrifera, Cornus canadensis, Picea glauca, } \\
\text { Equisetum silvaticum, and E. arvense commonly are present. }\end{array}$ \\
\hline $\begin{array}{l}\text { Fireweed (not } \\
\text { mapped) (Hfmf) }\end{array}$ & $\begin{array}{l}\text { Early successional communities dominated by Epilobium angustifolium with Calamagrostis } \\
\text { canadensis and Equisetum silvaticum. }\end{array}$ \\
\hline $\begin{array}{l}\text { Moist Graminoid, } \\
\text { post burn or } \\
\text { disturbance (Hgmd) }\end{array}$ & $\begin{array}{l}\text { Early successional communities affected by human activity. Vegetation includes indigenous } \\
\text { and introduced species including Hordeum jubatum, Achillea millifolium, Taraxacum spp., } \\
\text { Epilobium angustifolium, Poa spp., Festuca spp., and Calamagrostis canadensis. Scattered } \\
\text { shrubs may be present. }\end{array}$ \\
\hline $\begin{array}{l}\text { Halophytic Grass Wet } \\
\text { Meadow (Hgwhg) }\end{array}$ & $\begin{array}{l}\text { Active tidal flat communities dominated by salt tolerant grasses, primarily Puccinellia } \\
\text { nutkaensis. Triglochin maritimum, P. phryganodes, Atriplex gmelini, Plantago maritima, } \\
\text { and Salicornia europaea commonly are present. }\end{array}$ \\
\hline \multicolumn{2}{|c|}{$\begin{array}{l}\text { Halophytic Sedge Wet Active tidal flat communities dominated by Carex ramenskii. Potentilla egedii, Carex } \\
\text { Meadow, brackish lyngbyaei, and Triglochin maritimum are often present. } \\
\text { (Hgwhsb) }\end{array}$} \\
\hline $\begin{array}{l}\text { Halophytic Sedge W } \\
\text { Meadow, slightly } \\
\text { brackish (Hgwhss) }\end{array}$ & $\begin{array}{l}\text { Inactive tidal flat communities dominated by near pure stands of Carex lyngbyaei. } \\
\text { Associated species include Potentilla egedii, Triglochin maritimum, Carex aquatilis, } \\
\text { Scirpus paludosus, and Myriophyllum exalbescens. }\end{array}$ \\
\hline $\begin{array}{l}\text { Halophytic Sedge } \\
\text { Marsh (Hgwhsm) }\end{array}$ & $\begin{array}{l}\text { Semi-permanently flooded inactive tidal flats dominated by Scirpus paludosus. Associated } \\
\text { plants include } S \text {. validus, Zannichellia palustris, Hippuris tetraphylla, and Ruppia spiralis. }\end{array}$ \\
\hline $\begin{array}{l}\text { Subarctic Lowland } \\
\text { Sedge-Moss Bog } \\
\text { Meadow (Hgwsmb) }\end{array}$ & $\begin{array}{l}\text { Bogs and poor fens on lowland flats and depressions dominated by Sphagnum spp. and } \\
\text { sedges. Associated vegetation includes Menyanthes trifoliata, Carex aquatilis, C. } \\
\text { rotundata, C. rariflora, C. lasiocarpa, Andromeda polifolia, Oxycoccus microcarpus, and } \\
\text { Betula nana. }\end{array}$ \\
\hline
\end{tabular}

Mixed Herbs (Hfmm) Subalpine meadows dominated by Valeriana sitchensis and Geranium erianthum. Epilobium angustifolium, Heracleum lanatum, Veratrum viride, Sanguisorba stipulata, Calamagrostis canadensis, Cornus canadensis, Festuca altaica, and Artemisia tilesii are common associates.

Halophytic Herb Wet Coastal meadows on active tidal flats dominated by Triglochin maritimum. Associated Meadow (Hfwh) plants include Plantago maritima, Potentilla egedii, Lathyrus palustris, and Puccinellia nutkaensis.

Aquatic Herb (Hafl) Communities in permanently flooded depressions, shallow ponds and extensive pond margins with freshwater or salt tolerant aquatic species, such as Nuphar polysepalum, Zannichellia palustris, Hippuris tetraphylla, and Potamogeton species.

Barrens ( $<5 \%$ veg) Any area where vegetation covers less than $5 \%$ of the soil surface. Most common in the (Bbg) alpine, coast, on floodplains, and as a result of human disturbance. Colonizing species include lichens, graminoids, and salt tolerant forbs. See descriptions of Barren ecotypes (Table 9).

Partially Vegetated Sites where vegetation is poorly established and covers 5-30\% of the ground surface.

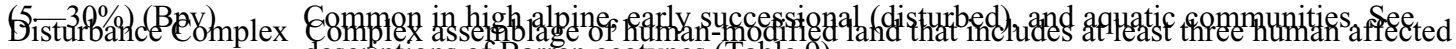
(DC)

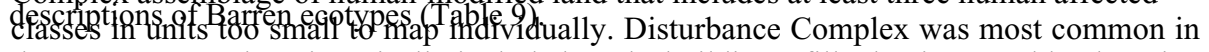
the cantonment where it typically included roads, buildings, fill, clearings, and landscaping.

Water (W) Freshwater lakes, streams, rivers and ponds with less than $5 \%$ cover of aquatic plants.

Brackish Water $(\mathrm{Wb})$ Waterbodies affected by both fresh and marine sources, salinity is intermediate between fresh water and seawater.

Marine Water (Wm) Nearshore waters of Knik Arm with salinity essentially unaffected by freshwater sources. 
Table 7. Areal extent of vegetation classes mapped on Fort Richardson, south-central Alaska, 2001.

\begin{tabular}{|c|c|c|c|}
\hline \multirow{2}{*}{$\underline{\text { Vegetation Class }}$} & \multicolumn{3}{|c|}{ Area } \\
\hline & acre & ha & $\%$ \\
\hline Open White Spruce & 1133 & 459 & 1.8 \\
\hline Open Black Spruce-White Spruce & 1429 & 578 & 2.3 \\
\hline Open Black Spruce & 701 & 284 & 1.1 \\
\hline Closed Quaking Aspen & 128 & 52 & 0.2 \\
\hline Closed Paper Birch-Aspen & 1467 & 594 & 2.4 \\
\hline Closed Paper Birch & 5006 & 2026 & 8.1 \\
\hline Open Balsam Poplar & 529 & 214 & 0.9 \\
\hline Open Quaking Aspen & 213 & 86 & 0.3 \\
\hline Open Paper Birch-Aspen & 1004 & 406 & 1.6 \\
\hline Open Paper Birch & 3526 & 1427 & 5.7 \\
\hline Open Black Cottonwood & 318 & 128 & 0.5 \\
\hline Closed Quaking Aspen-Spruce & 129 & 52 & 0.2 \\
\hline Closed Spruce-Paper Birch & 1167 & 472 & 1.9 \\
\hline Open Quaking Aspen-Spruce & 1417 & 574 & 2.3 \\
\hline Open Spruce-Paper Birch & 16,240 & 6572 & 26.2 \\
\hline Open Black Cottonwood-White Spruce & 312 & 126 & 0.5 \\
\hline Closed Dwarf Mountain Hemlock & 120 & 49 & 0.2 \\
\hline Open Dwarf Black Spruce & 299 & 121 & 0.5 \\
\hline Closed Tall Willow & 89 & 36 & 0.1 \\
\hline Closed Tall Alder & 2198 & 890 & 3.5 \\
\hline Closed Tall Scrub, post burn or disturbance & 8 & 3 & $<0.1$ \\
\hline Open Tall Willow & 318 & 129 & 0.5 \\
\hline Open Tall Alder & 1866 & 755 & 3.0 \\
\hline Open Tall Scrub, post burn or disturbance & 576 & 233 & 0.9 \\
\hline Closed Low Willow & 32 & 13 & 0.1 \\
\hline Open Low Willow & 148 & 60 & 0.2 \\
\hline Open Low Shrub Birch-Willow & 1559 & 631 & 2.5 \\
\hline Open Low Sweetgale-Graminoid Shrub Meadow, slightly brackish & 153 & 62 & 0.2 \\
\hline Open Low Shrub Birch-Ericaceous Shrub Bog & 628 & 254 & 1.0 \\
\hline Open Low Sweetgale-Graminoid Bog & 76 & 31 & 0.1 \\
\hline Open Low Scrub, post burn or disturbance & 1563 & 632 & 2.5 \\
\hline Dryas-Lichen Tundra & 4946 & 2002 & 8.0 \\
\hline Cassiope Tundra & 198 & 80 & 0.3 \\
\hline Crowberry Tundra & 3188 & 1290 & 5.1 \\
\hline Elymus & 16 & 6 & $<0.1$ \\
\hline Bluejoint Meadow & 69 & 28 & 0.1 \\
\hline Moist Graminoid, post burn or disturbance & 667 & 270 & 1.1 \\
\hline Halophytic Grass Wet Meadow & 83 & 34 & 0.1 \\
\hline Halophytic Sedge Wet Meadow, brackish & 187 & 76 & 0.3 \\
\hline Halophytic Sedge Wet Meadow, slightly brackish & 359 & 145 & 0.6 \\
\hline Halophytic Sedge Marsh & 299 & 121 & 0.5 \\
\hline Subarctic Lowland Sedge-Moss Bog Meadow & 190 & 77 & 0.3 \\
\hline Mixed Herbs & 190 & 77 & 0.3 \\
\hline Halophytic Herb Wet Meadow & 273 & 110 & 0.4 \\
\hline Aquatic Herb & 40 & 16 & 0.1 \\
\hline Barrens $(<5 \%$ veg $)$ & 1890 & 765 & 3.1 \\
\hline Partially Vegetated (5-30\%) & 3161 & 1279 & 5.1 \\
\hline Disturbance Complex & 1272 & 515 & 2.1 \\
\hline Water & 405 & 164 & 0.7 \\
\hline Brackish Water & 182 & 73 & 0.3 \\
\hline Marine Water & 26 & 11 & $<0.1$ \\
\hline Total & 61,996 & 25,089 & 100 \\
\hline
\end{tabular}




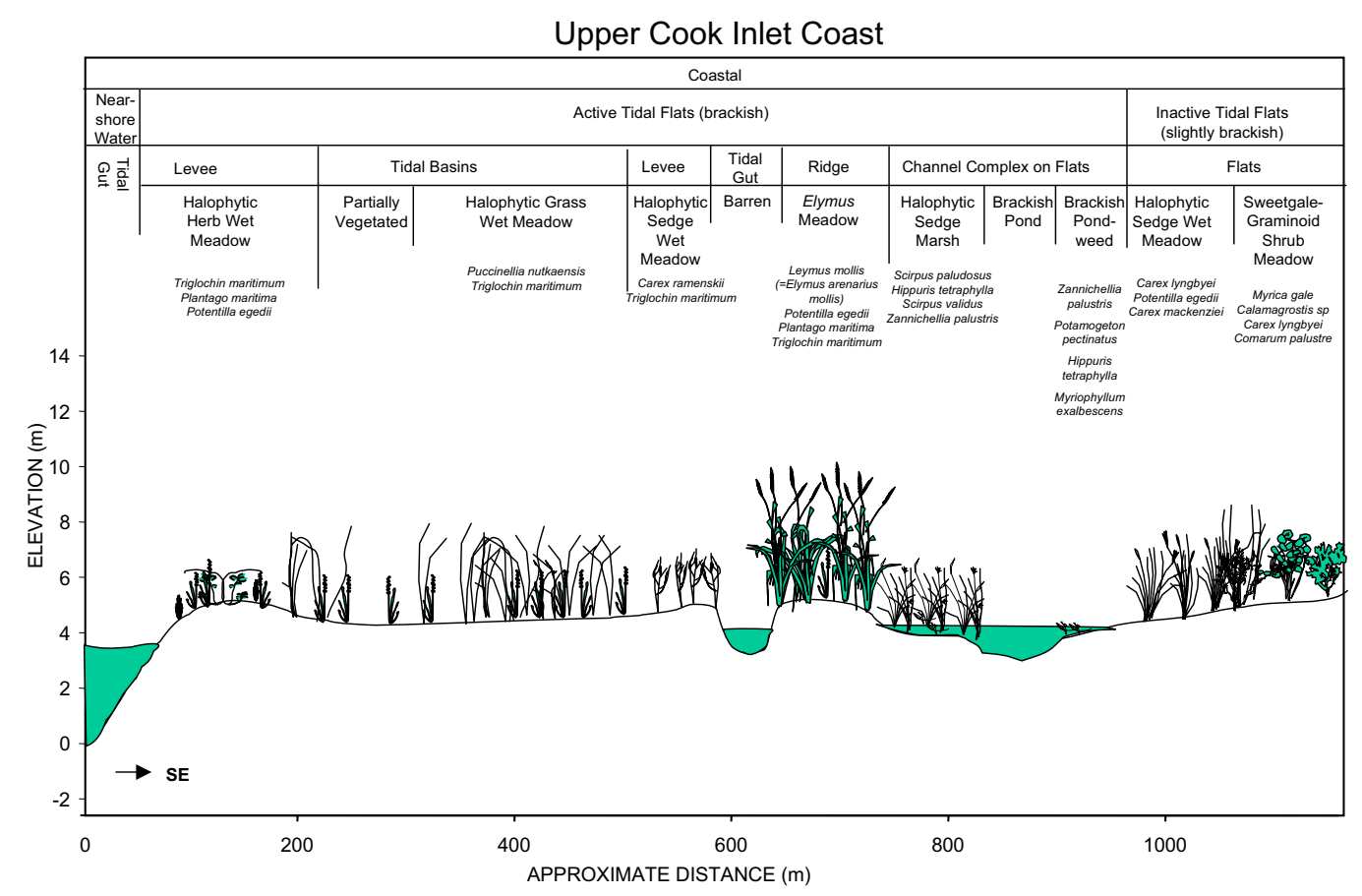

Figure 10. Toposequence on the Eagle River Flats along the Upper Cook Inlet Coast illustrating geomorphology, vegetation, elevations, and soil stratigraphy, Fort Richardson, south-central Alaska, 2001.

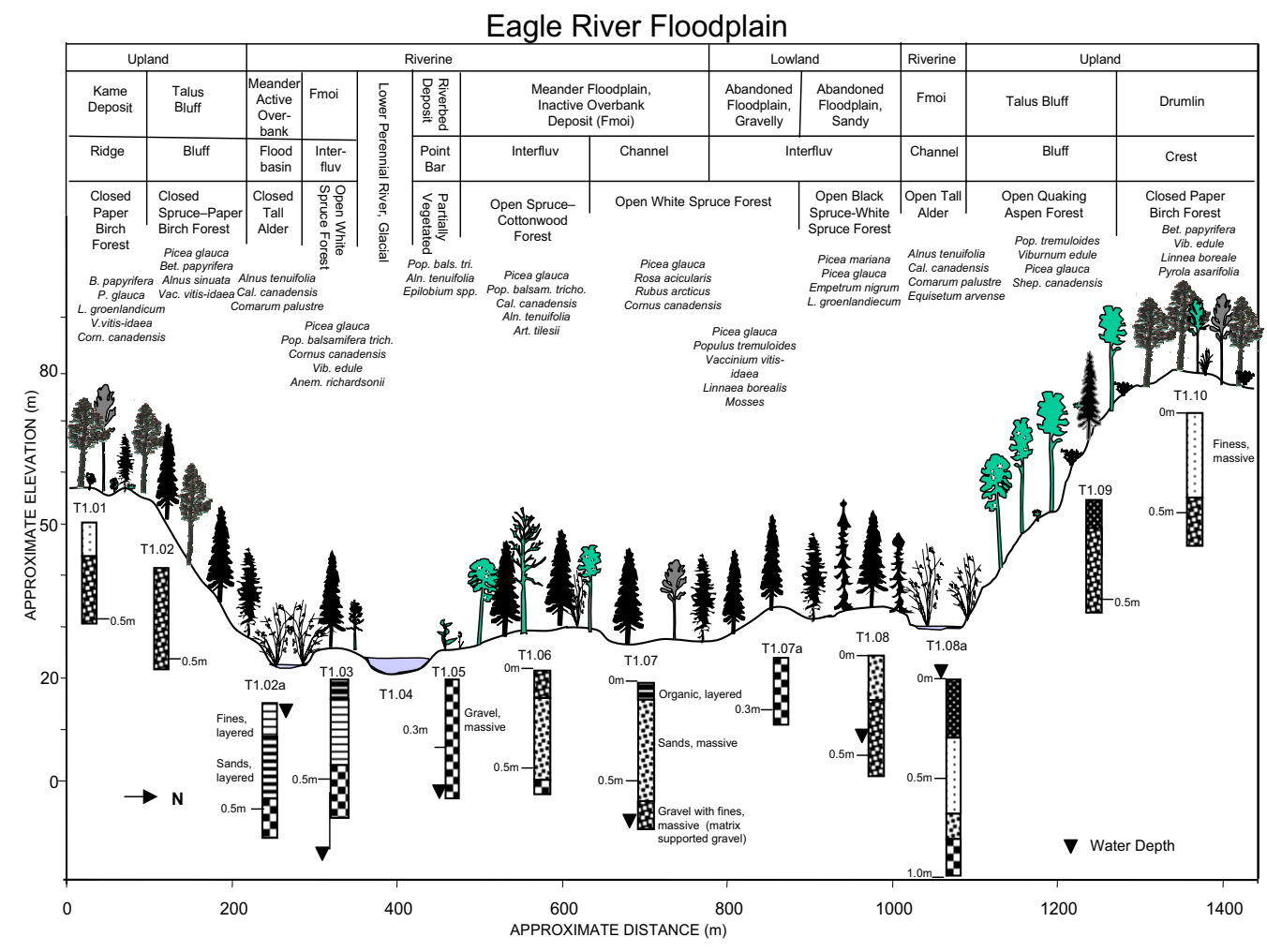

Figure 11. Toposequence (Transect 1) on the Eagle River Floodplain illustrating geomorphology, vegetation, elevations, and soil stratigraphy, Fort Richardson, south-central Alaska, 2001. 


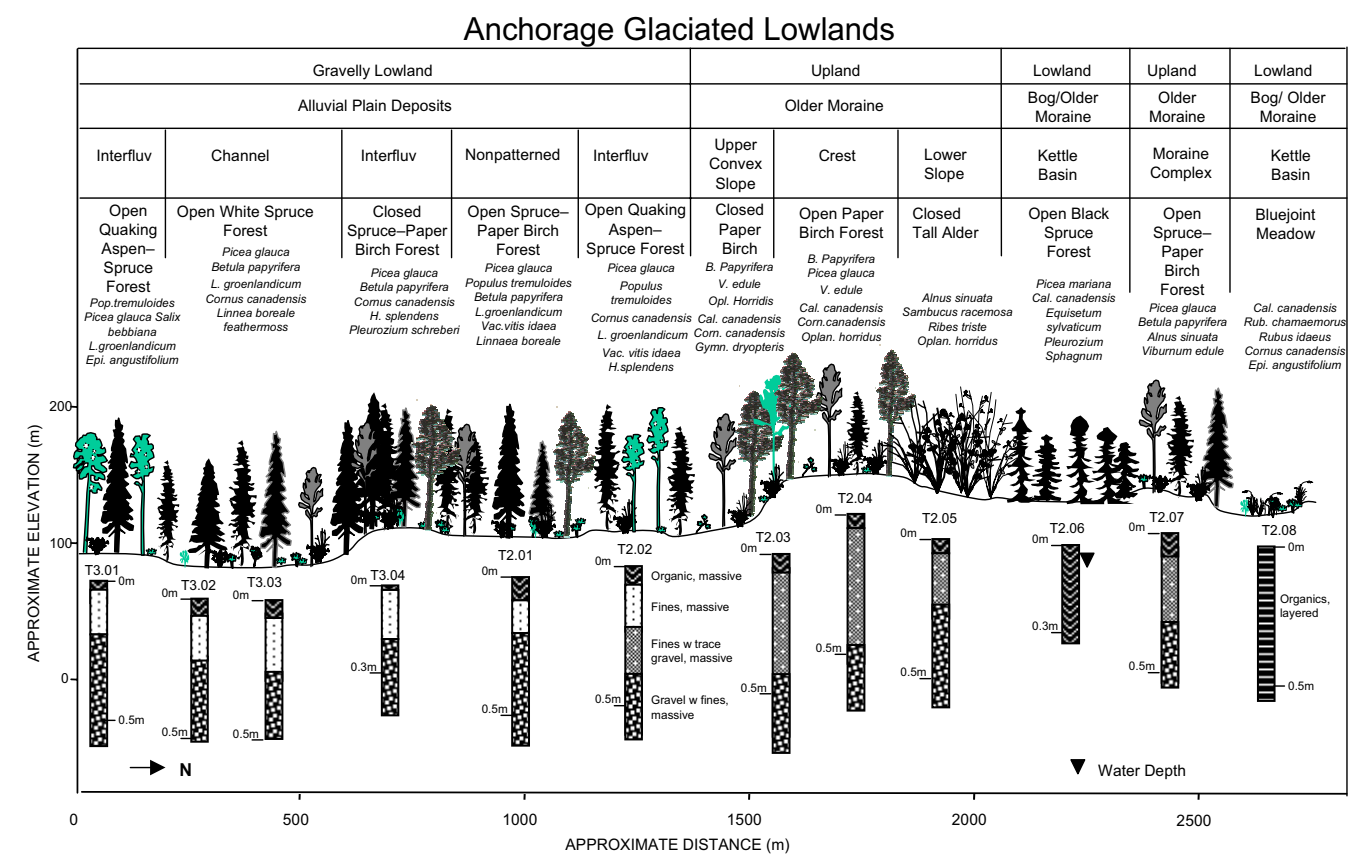

Figure 12. Toposequence (Transect 2) near Camp Carrol in the Anchorage Glaciated Lowlands illustrating geomorphology, vegetation, elevations, and soil stratigraphy, Fort Richardson, south-central Alaska, 2001.

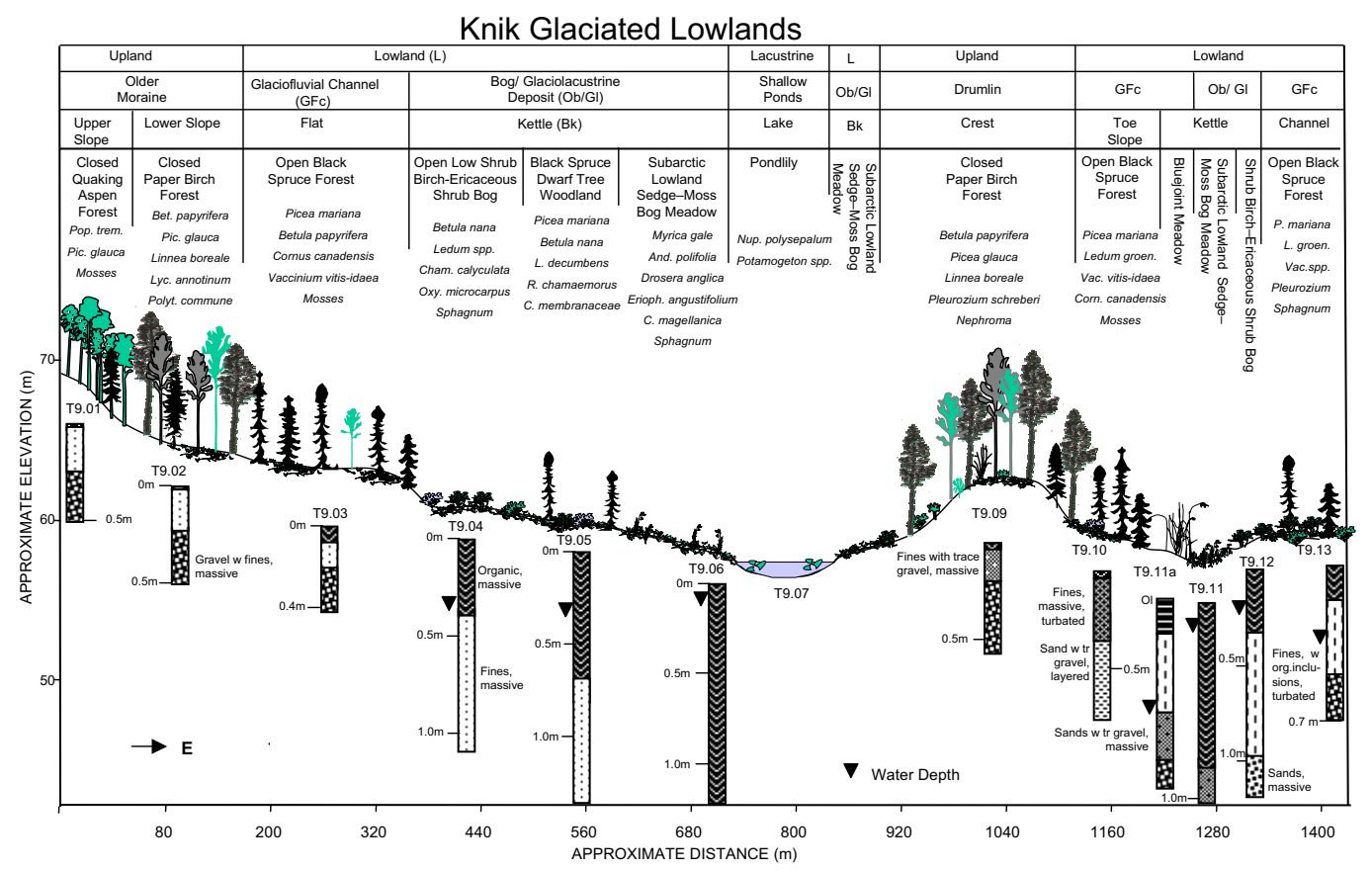

Figure 13. Toposequence (Transect 9) near Chain Lake in the Knik Glaciated Lowlands illustrating geomorphology, vegetation, elevations, and soil stratigraphy, Fort Richardson, south-central Alaska, 2001. 


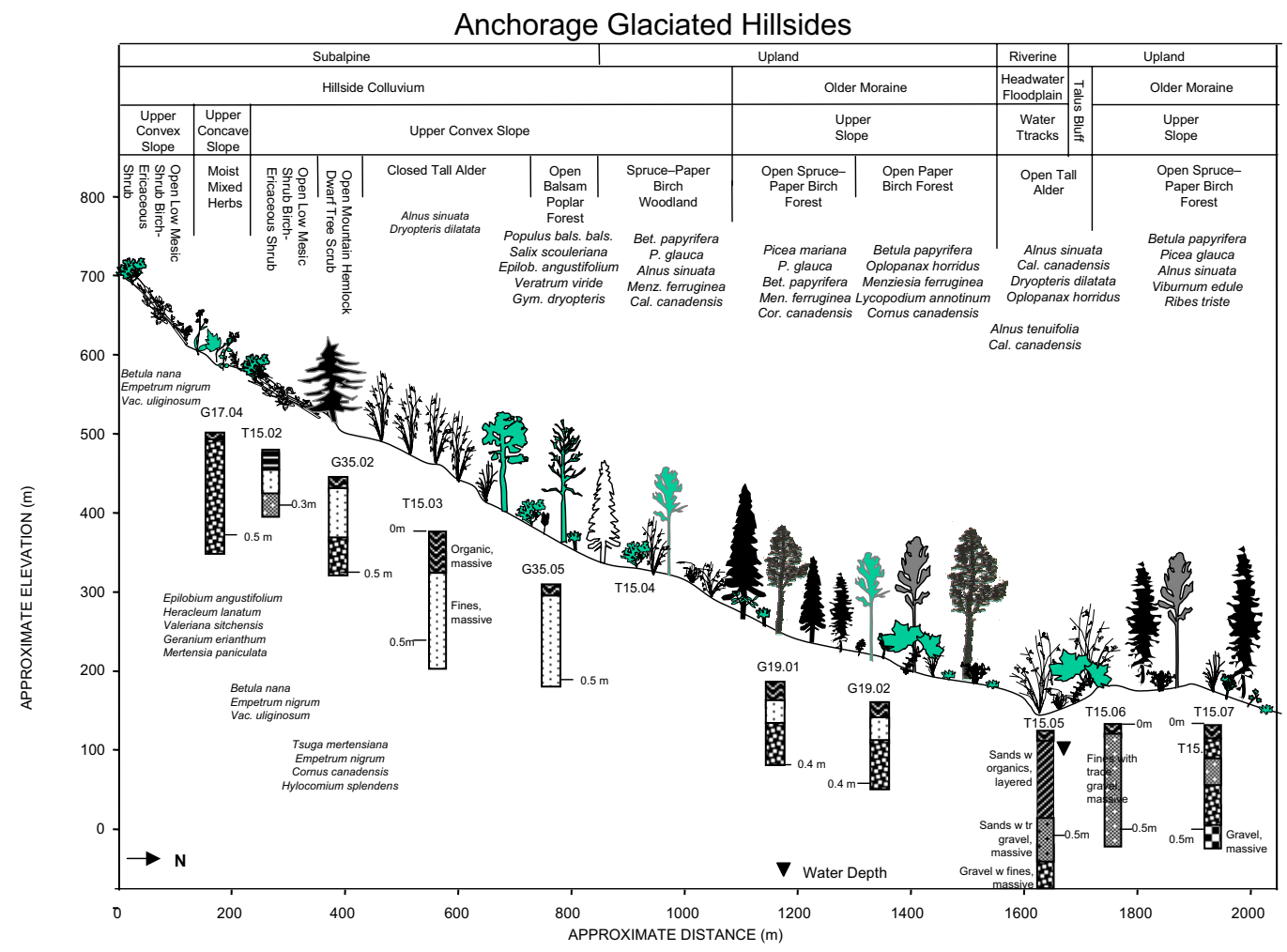

Figure 14. Toposequence (Transect 15) on the Anchorage Glaciated Hillsides illustrating geomorphology, vegetation, elevations, and soil stratigraphy, Fort Richardson, south-central Alaska, 2001.

Northern Chugach Mountains

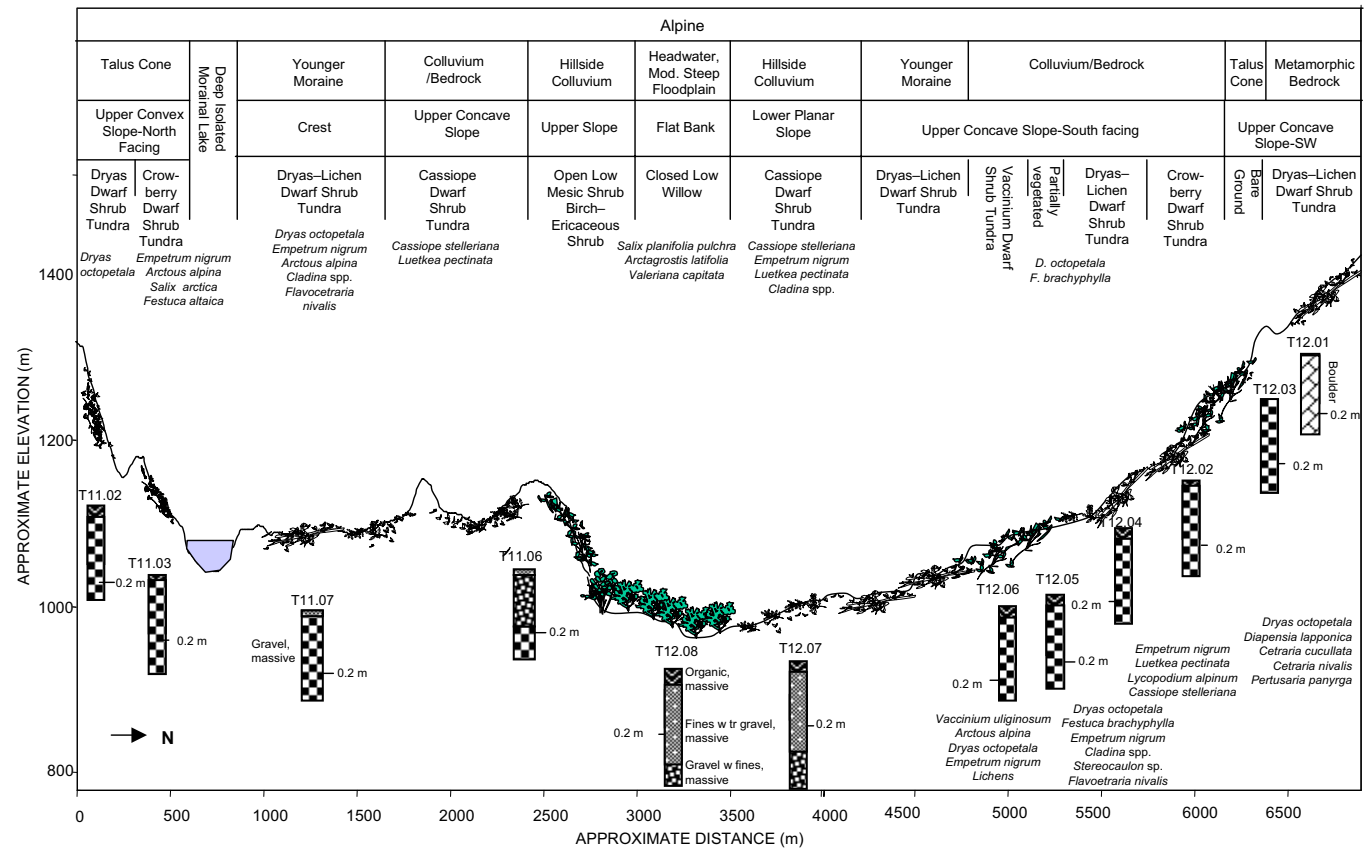

Figure 15. Toposequence (Transects 11 and 12) in Snowhawk Valley in the Northern Chugach Mountains illustrating geomorphology, vegetation, elevations, and soil stratigraphy, Fort Richardson, south-central Alaska, 2001. 
moderately well-drained, mineral soils supported Bluejoint Meadow. Bog deposits in depressions with wet, organic-rich soils supported scrub and meadow vegetation, typically Open Low Shrub Birch-Ericaceous Shrub Bog and Subarctic Lowland Sedge-Moss Bog Meadow. A few ponds occurred in depressions that had not yet filled in with peat, the vegetation in these consisted of aquatic herbs such as Pondlily, Nuphar polysepalum.

On the Anchorage Glaciated Hillsides of the Eklutna Mountains (portion of the Chugach Mountains near Anchorage), the geomorphology was dominated by Hillside Colluvium and Older Moraine deposits (Fig. 14). The Hillside Colluvium had well-drained, rocky soils (occasionally with thin loamy horizons) that supported a wide range of subalpine, upland, and riverine vegetation types. In the subalpine zone, the vegetation was dominated by Open Balsam Poplar Forest, Open Mountain Hemlock Dwarf Tree Scrub, Closed Tall Alder, Open Low Mesic Shrub Birch-Ericaceous Shrub, and Mixed Herbs. Lower on the slope, the upland areas were dominated by Open Spruce-Paper Birch Forest and Open Paper Birch Forest. Headwater floodplains in narrow valleys and gullies supported Open Tall Alder and Open Tall Willow.

At upper elevations in the Chugach Mountains, the geomorphology was dominated by Metamorphic Bedrock, Talus Cones, Hillside Colluvium, Younger Moraine, and Moderately Steep Headwater Floodplains (Fig. 15). Metamorphic Bedrock occurred as rock outcrops or on ridges with excessively drained, shallow rocky soils. These areas usually were barren except for some crustose lichens, and occasionally Dryas-Lichen Dwarf Shrub Tundra. Talus cones usually consisted of barren rubble, but occasionally supported Dryas Dwarf Shrub Tundra and Crowberry Dwarf Shrub Tundra in more stable areas. Younger Moraine and Hillside Colluvium were dominated by Dryas-Lichen Dwarf Shrub Tundra, Crowberry Dwarf Shrub Tundra, Vaccinium Dwarf Shrub Tundra, and Open Low Mesic Shrub Birch-Ericaceous Shrub, depending on exposure to wind and slope position. Headwater Floodplains in the alpine zone typically had Closed Low Willow scrub.

Relationships among ecological components Hierarchical relationships among ecosystem components were developed by successively grouping data from survey plots by climate, physiography, soil texture, geomorphology, slope position, drainage, vegetation structure, and vegetation composition (Table 8). Frequently, geomorphic units with similar texture or genesis were grouped (e.g., sandy and gravelly textures were grouped for some lowlands) to reduce the number of classes. Ecotypes then were derived from these tabular associations of primary ecological components.

This hierarchical grouping revealed that there were close associations among soil texture, geomorphology, slope position, drainage, and vegetation. Usually, there were several geomorphic units with similar soil characteristics that were associated with several closely related plant associations. These plant associations generally were closely related because they represented stages in a post-disturbance successional sequence. For example, the sequence from herb-moss through tall scrub, broadleaf forest, and mixed forest to needleleaf forest is the typical succession of vegetation development after fire in Alaska (Foote 1983, Viereck et al. 1983). Usually there was a one-to-one correspondence between plant associations and ecotypes, except for forest classes where changes in dominance of trees in the canopy (shift from broadleaf to mixed and coniferous) did not lead to consistent changes in associated plants in the understory. For example, upland needleleaf and mixed forests shared a plant association, while upland and lowland broadleaf forests shared a plant association.

The successive grouping of ecosystem components and identification of landscape relationships helped differentiate forest types during mapping. For example, aspen often was associated with south-facing upland slopes and gravelly lowlands, while black cottonwood generally was restricted to riverine areas. Birch, white spruce, and black spruce, however, occurred over a wide range of conditions. For more detailed presentation of floristic differences among ecotypes see the discussion of vegetation composition in the Ecotypes section.

An important question is how well these general relationships conform to the data set and whether they can be used reliably to extrapolate trends across the landscape. During cross-tabulation of the ecological relationships, 25\% (58/232) of field observations were excluded from the relationships table because they were rare occurrences that didn't conform to the more frequent associations identified among geomorphology, texture, drainage, vegetation structure, or plant association. Most of the inconsistencies between individual plots and the typical characteristics of the ecotype class resulted from differences in soil texture (14\% excluded). Frequently, upland or riverine sites had surface layers of loam or sand that were slightly thicker than the 50$\mathrm{cm}$ criteria used to establish the dominant texture for the site. In contrast, the vegetation structure $(2 \%$ excluded) and plant association ( $7 \%$ excluded) classes for the plots were highly consistent with the final ecotype designations. Similarly, geomorphic units (4\% excluded) were highly associated with the vegetation and final ecotype designations.

The level of inconsistency in landscape relationships 


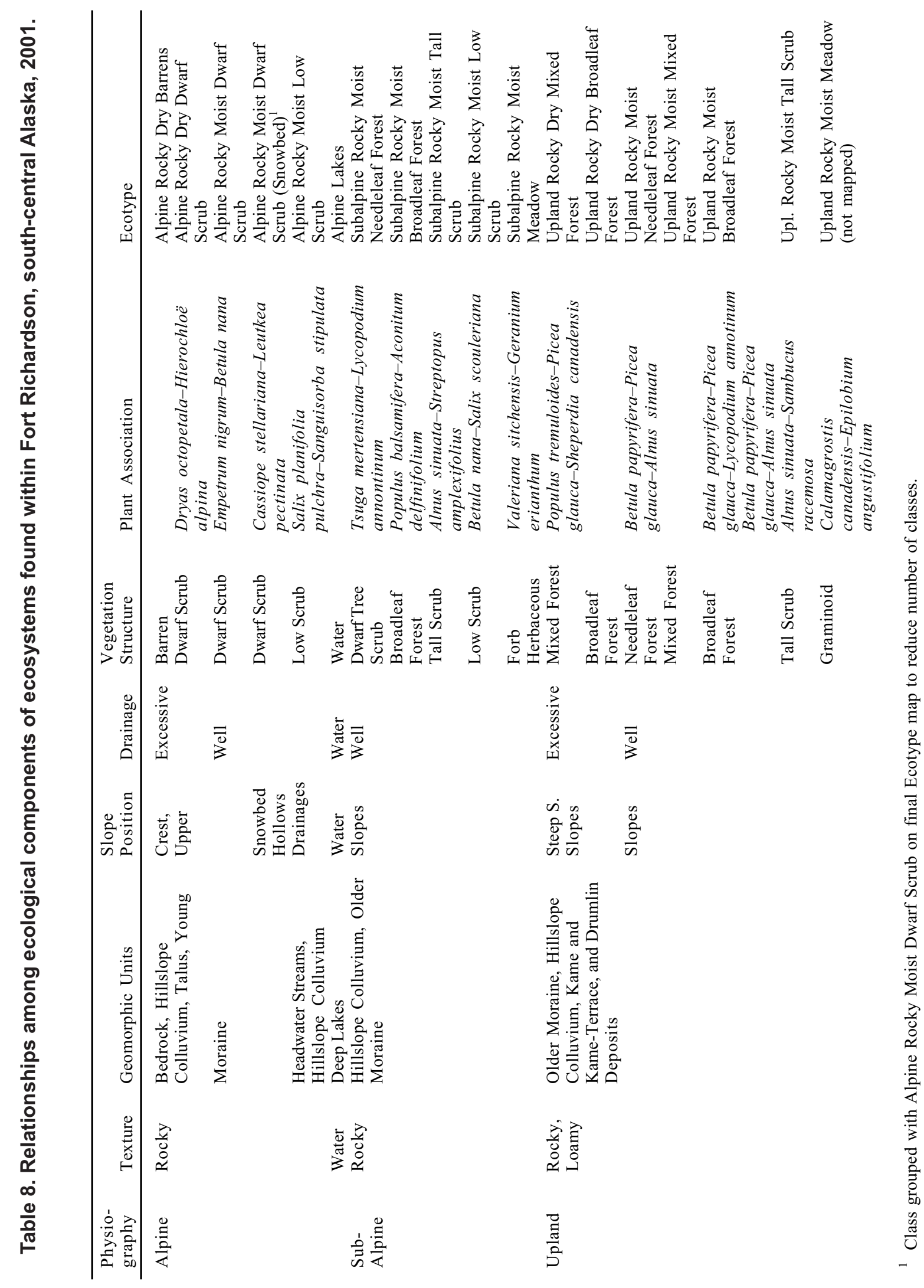




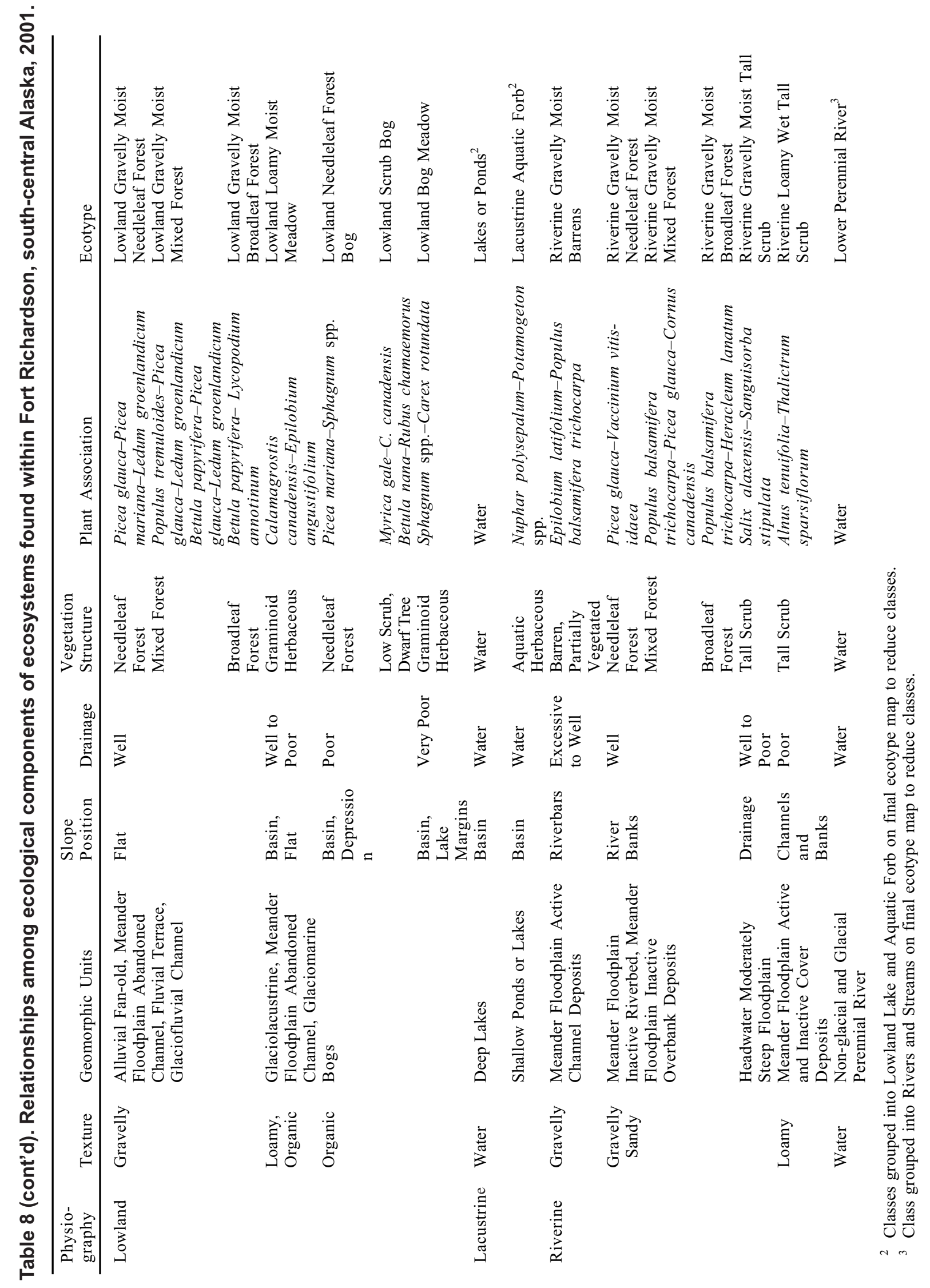




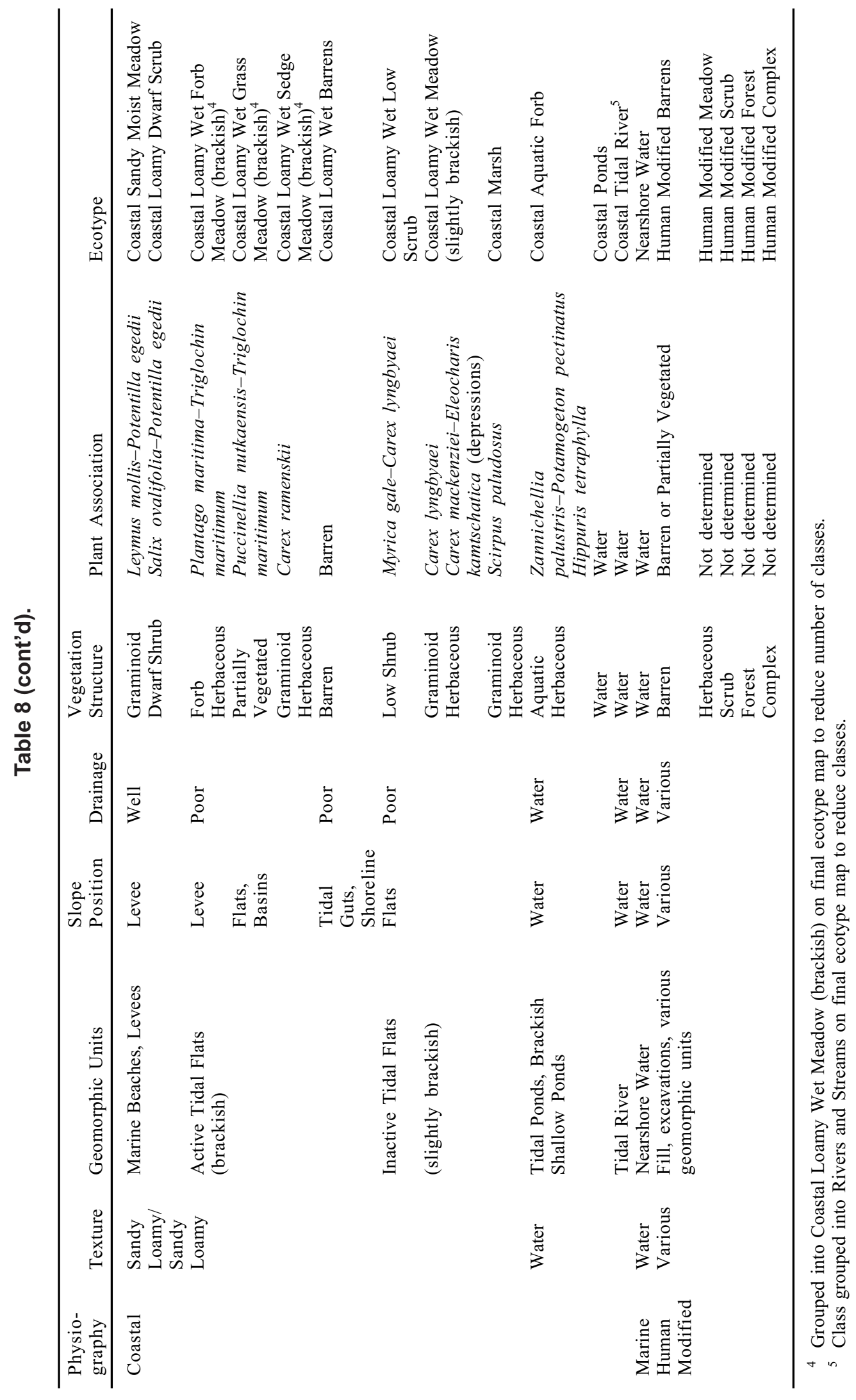


was similar to those observed at Fort Greely (25\% excluded, Jorgenson et al. 2001) and at Fort Wainwright (17\% excluded, Jorgenson et al. 1999). We attribute the observed inconsistencies to (1) the complexity in the distribution of thin loess deposits over glaciated terrain, which made the distribution of soil properties extremely patchy; (2) a substantial elevation range, which creates multiple transition zones from closed canopy forests to woodland forests to alpine shrublands; and (3) the relatively high diversity of geomorphic units and vegetation characteristics associated with terrain that extends from the coast to rugged mountains. In developing ecotype classes and landscape relationships we focused on preserving distinct patterns and trends rather than including all plots, in order to minimize confusion among classes. This reflects our belief that no classification system can completely describe landscape patterns; some proportion (in this case $25 \%$ ) of sites will be difficult to classify because they are transitional (ecotones) or have been affected by historical factors (e.g., change in water levels, disturbances) that affect vegetation response to current environmental conditions. The occurrence of these inconsistencies provides an upper limit for the accuracy of mapping of about $75 \%$, because a certain portion of the landscape will not fit readily into any of the classes.

The advantage of this hierarchical approach, combining physiography and vegetation structure, is that the resulting classes are effective at differentiating both vegetation composition and soil characteristics. This approach is particularly useful for mapping based on photo interpretation, because physiography (e.g., flat lowlands versus hilly uplands) and vegetation structure (e.g., needleleaf trees, broadleaf trees, shrubs, and graminoids) can usually be distinguished easily on aerial photographs. Distinguishing species of trees (e.g., birch versus poplar) or shrubs (e.g., dwarf birch versus willow) from photographs is much more difficult. Finally, the linkage of soil characteristics to ecotypes is important for differentiating ecotypes that may have different sensitivities to disturbance. For example, most ecotypes on Fort Richardson have rocky soils, which increases their resistance to traffic and reduces potential for erosion, whereas loamy or organic soils are much more sensitive to off-road traffic.

The main disadvantage to this integrated approach is that physiography or slope position, which is an important component of the classification system, contributes to uncertainty in classification and mapping in some situations. While alpine (above treeline), subalpine (near treeline), riverine (near rivers), and coastal (salt-affected) ecosystems can be distinguished easily, the difference between upland and lowland physiography is particularly problematic in broad transitional areas. In addition, upland/lowland differences are scaledependent (e.g., a small raised area seen on the ground may function as an upland even though it occurs within a broad lowland area). This problem with differentiation of physiography is similar to that associated with the hydrogeomorphic classes (e.g., slopes, depressions, flats) developed by Brinson (1993). A second disadvantage of the integrated approach is that the grouping of the many ecological components can lead to generation of a large number of classes. For practical purposes, the number of classes must be reduced by combining similar characteristics and ignoring unusual plots that do not fit well in any class.

\section{Ecotypes}

Classification and mapping Ecological relationships among geomorphic, surface form, and vegetation characteristics of the landscape were analyzed to derive 51 ecotypes for Fort Richardson (Fig. 16 and 17, Table 9). The ecotypes were grouped into six alpine, five subalpine, seven upland, seven lowland, two lacustrine, six riverine, twelve coastal, one marine, and five human-modified classes. For final mapping the number of ecotypes was reduced to 46 by aggregating closely related types; this eliminated several classes that could not be reliably mapped (Fig. 18, Table 10). The physical and biological characteristics of each ecotype are described in Table 9.

The most abundant ecotypes were upland forest types, predominantly Upland Rocky Moist Mixed Forest $(22.2 \%)$, and Upland Rocky Moist Broadleaf Forest (15.4\%). In the lowlands, the most abundant type was Lowland Gravelly Moist Mixed Forests (7.2\%). The most common subalpine ecotypes were Rocky Moist Tall Scrub (4.5\%) and Subalpine Rocky Moist Low Scrub (2.5\%). Alpine ecotypes were widespread, and included Alpine Rocky Dry Dwarf Scrub (8.0\%), Alpine Rocky Moist Dwarf Scrub (5.5\%), and Alpine Rocky Dry Barrens (4.3\%). Coastal ecotypes were uncommon, and consisted mainly of Coastal Loamy Wet Barrens (1.1\%) and Coastal Loamy Brackish Wet Meadows $(0.9 \%)$. Riverine ecotypes also were rare; the most common riverine type was Riverine Gravelly Moist Mixed Forest (0.8\%). Human modified ecotypes covered $12.9 \%$ of the area and consisted primarily of $\mathrm{Hu}-$ man Modified Scrub (5.3\%) and Human Modified Barrens $(2.6 \%)$.

The large number of ecotype classes reflects the high ecological diversity on Fort Richardson, which results from strong environmental gradients from the coast to the alpine areas. The initial ecotype classification of the preliminary map identified a potential set of 89 ecotypes derived from combinations of 503 integrated terrain units. We consolidated closely related types and 

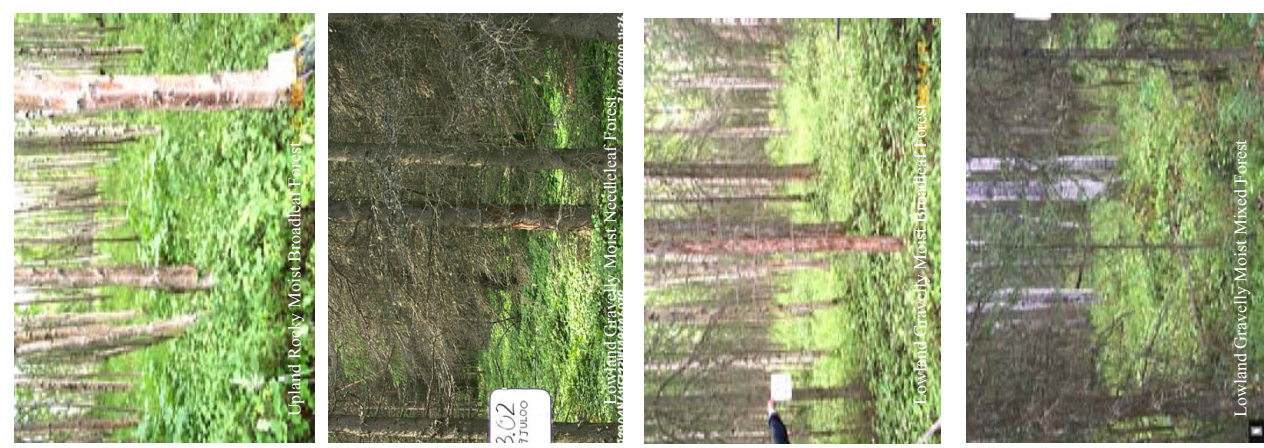

这
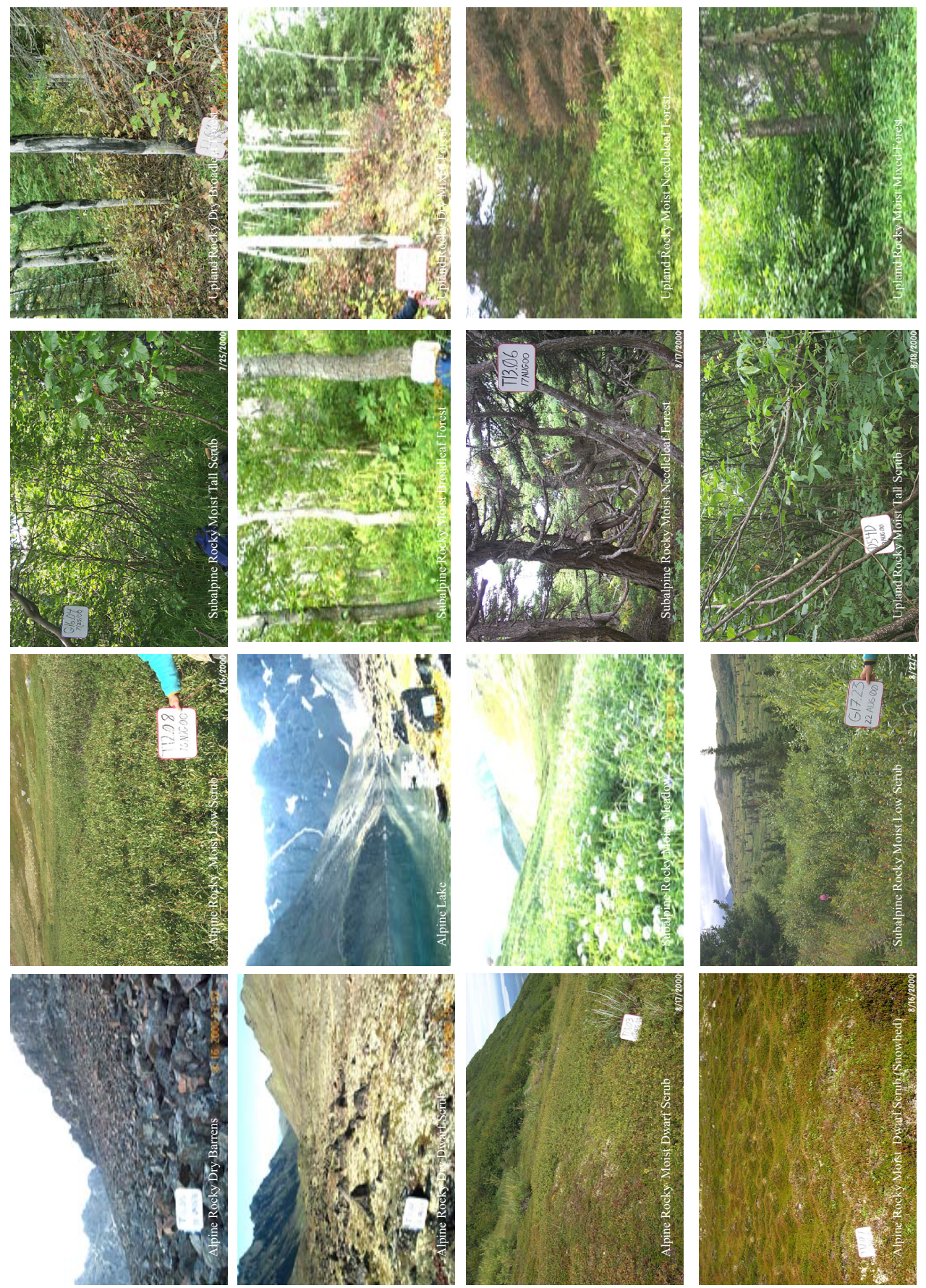

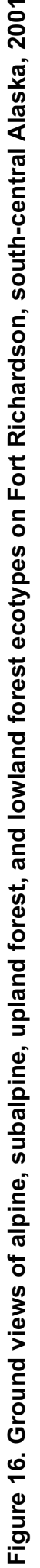



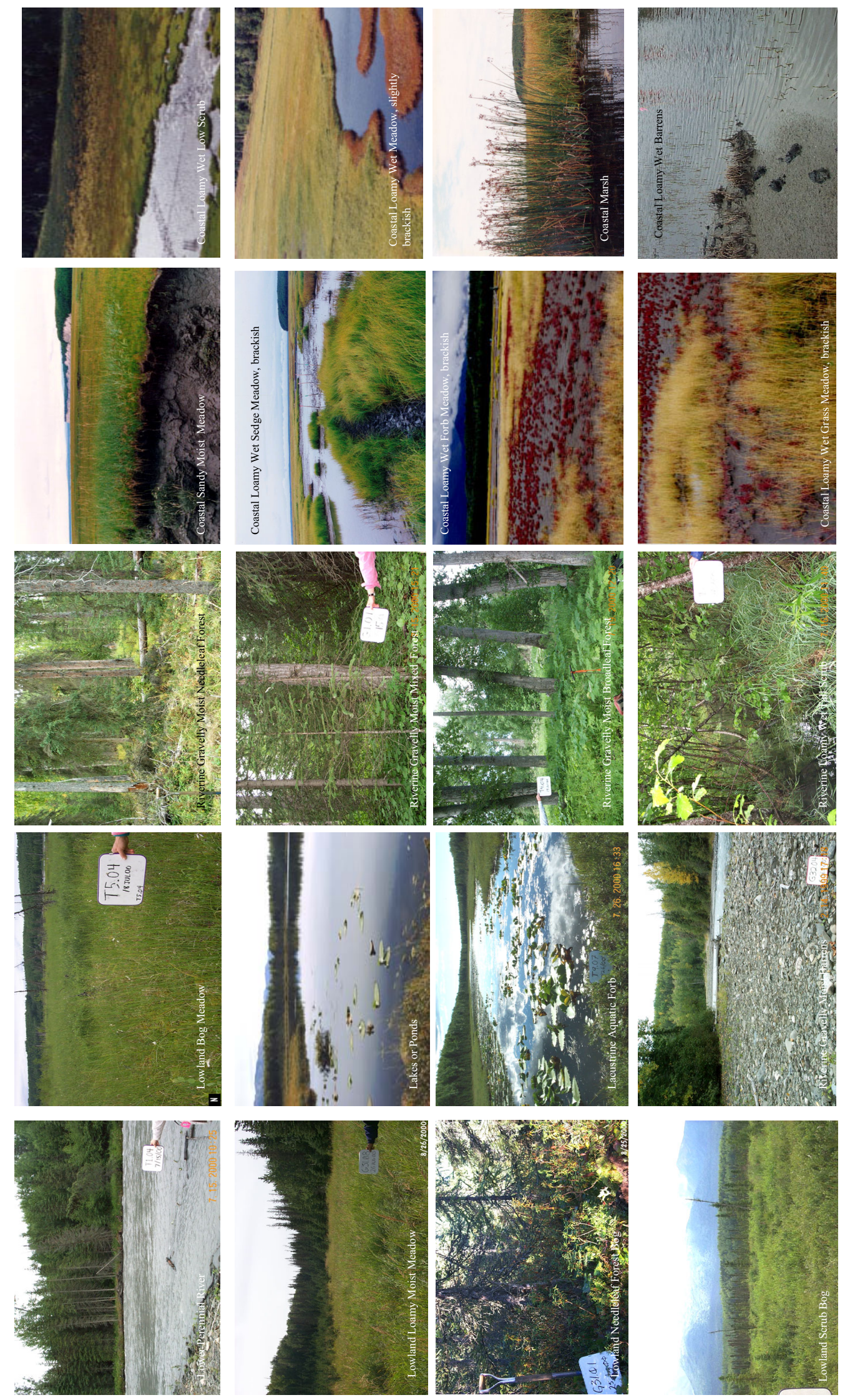

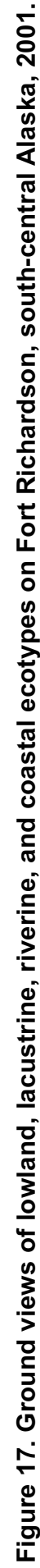


Table 9. Classification and description of ecotypes found within Fort Richardson, south-central Alaska, 2001. Descriptions include physiography, geomorphology, soils properties, and vegetation. Plant names in bold indicate the dominant and differential plants used to define the related plant association.

Class

Description

Alpine Rocky

Dry Barrens

Alpine Rocky

Dry Dwarf

Scrub

Alpine Rocky Moist Dwarf

Scrub

Alpine Rocky

Moist Dwarf

Scrub

(snowbed)

Alpine Rocky

Moist Low

Scrub

Alpine Lake

Subalpine

Rocky Moist

Meadow

Subalpine

Rocky Moist

Low Scrub

Subalpine

Rocky Moist

Tall Scrub

Subalpine

Rocky Moist

Broadleaf

Forest
Rugged, wind-swept, barren or partially vegetated $(<30 \%$ cover $)$ areas on exposed bedrock ridges or unstable talus slopes above treeline $(>800 \mathrm{~m})$. Soils are rocky, dry, excessively drained, lacking in an organic horizon, and strongly acidic. Scattered, prostrate species may be present including Dryas octopetala, Salix arctica, Saxifraga bronchialis, Cetraria nivalis, Cladonia spp. Thamnolia sp., and diverse crustose lichens.

Moderate to steep slopes and crests on exposed colluvium, old moraine, or talus above treeline, with vegetation dominated by the dwarf $(<0.2 \mathrm{~m})$ evergreen shrub, Dryas octopetala, cushion plants, and lichens. Soils are rocky, dry, excessively to well-drained, with little or no organic horizon, and acidic. Common plants include Hierochlo alpina, Salix arctica, Empetrum nigrum, Diapensia lapponica, Vaccinium vitis-idaea, Oxytropis bryophila, Carex michrochaeta, Cetraria nivalis, Alectoria ochroleuca, Bryocaulon divergens, Cladina arbuscula, C. stellaris, Stereocaulon sp. and crustose lichens. This is the dominant alpine ecotype, it forms the vegetated zone immediately below Alpine Rocky Dry Barrens and often intergrades with that class.

Moderate mountain slopes above treeline on colluvium or talus slopes with vegetation dominated by the dwarf shrub Empetrum nigrum, and prostrate Betula nana. Soils are rocky, moist, well to somewhat poorly drained, with a moderate to shallow organic horizon, and strongly acidic. At elevations immediately below Alpine Rocky Dry Dwarf Scrub this class is dominated by E. nigrum, farther downslope and in more protected areas $B$. nana becomes more common and is a co-dominate at elevations approaching subalpine. Associated plants include Vaccinium uliginosum, V. vitis-idaea, Arctous alpina, and Cornus canadensis.

Protected sites above treeline on colluvium in snowbed hollows and concave slopes with vegetation dominated by the dwarf shrubs Cassiope stelleriana and Luetkea pectinata. Soils are rocky, moist, well-drained, with moderate to shallow organic horizons, and strongly acidic. Associated plants include Empetrum nigrum, Lycopodium alpinum, Huperzia selago, Gentiana glauca, and Cladina stellaris. This class was consolidated with Alpine Rocky Moist Dwarf Scrub for map presentation.

This class occurs on steep headwater floodplains above treeline and has vegetation dominated by low $(0.2-1.5 \mathrm{~m})$ willows (Salix spp.). Soils are rocky, moist, well-drained, with a shallow organic horizon, and acidic. Common species include Salix planifolia pulchra, Salix barclayi, Sanguisorba stipulata, Veratrum viride, Epilobium angustifolium, and Calamagrostis canadensis.

Oligotrophic waterbodies above treeline with neutral $\mathrm{pH}$. Water is deep $(>1.5 \mathrm{~m})$.

Upper, moderate to gentle, concave slopes on colluvium or older moraine at, or immediately above, treeline with lush vegetation dominated by forbs. Soils are rocky, well-drained, moist, with a shallow organic horizon, and acidic. Common species include Geranium erianthum, Valeriana sitchensis, Epilobium angustifolium, Heracleum lanatum, Veratrum viride, Sanguisorba stipulata, Calamagrostis canadensis, Cornus canadensis, Festuca altaica, and Artemisia tilesii.

Moderate or steep upper slopes on colluvium or old moraine at treeline with diverse vegetation dominated by low and tall deciduous shrubs, dwarf shrubs, and forbs. Soils are rocky, well- to moderately well-drained, moist, with a shallow to moderate organic horizon, and acidic. Betula nana, Salix scouleriana, Empetrum nigrum, and Pleurozium schreberi are common dominants. Associated plants include Calamagrostis canadensis, B. occidentalis, Rosa acicularis, Festuca altaica, Epilobium angustifolium, Aconitum delphinifolium, Vaccinium uliginosum, Cornus canadensis, and Geranium erianthum.

Broad, steep, upper slopes at treeline with vegetation dominated by Alnus sinuata and ferns. Soils are rocky, well-drained, moist, with a shallow to moderate organic horizon, and acidic. This class forms a nearly continuous band of vegetation through the subalpine zone and is the dominant subalpine ecotype. Associated species include Streptopus amplexifolius, Gymnocarpium dryopteris, Dryopteris dilitata, and Calamagrostis canadensis.

Upper south-facing slopes on colluvium or older moraine at treeline, vegetation is dominated by Populus balsamifera balsamifera, low shrubs, and forbs. Trees may be severely stunted on exposed sites. Soils are rocky, well-drained, moist, with a shallow to moderate organic horizon, and acidic. Associated plants include Cornus canadensis, Aconitum delphinifolium, Viburnum edule, Veratrum viride, Heracleum lanatum, Geranium erianthum, Salix scouleriana, and Epilobium angustifolium. 
Table 9 (cont'd). Classification and description of ecotypes found within Fort Richardson, southcentral Alaska, 2001. Descriptions include physiography, geomorphology, soils properties, and vegetation. Plant names in bold indicate the dominant and differential plants used to define the related plant association.

Class Description

Subalpine

Rocky Moist

Needleleaf

Forest

Upland Rocky

Dry Barrens

Upland Rocky

Dry Broadleaf

Forest

Upland Rocky

Dry Mixed

Forest

Upland Rocky

Moist Meadow

(not mapped)

Upland Rocky

Moist Tall

Scrub

Upland Rocky

Moist

Broadleaf

Forest

Upland Rocky

Moist Mixed

Forest

Upland Rocky

Moist

Needleleaf

Forest

Lowland Loamy Moist

Meadow

Lowland Gravelly Moist Tall Scrub

Lowland

Gravelly Moist

Broadleaf

Forest
Moderate to steep slopes on colluvium or old moraine at treeline $(\sim 600-800 \mathrm{~m})$ with vegetation dominated by the dwarf tree Tsuga mertensiana and forbs and mosses in the understory. Soils are rocky, well-drained, moist, with a shallow to moderate organic horizon, and acidic. Associated plants include Cornus canadensis Empetrum nigrum, Lycopodium annotinum, Gymnocarpium dryopteris, Linnaea borealis, Rubus pedatus, Calamagrostis canadensis, and Pleurozium schreberi.

Unvegetated or partially vegetated $(<30 \%$ cover $)$ bluffs and unconsolidated upland soils. Slopes usually are steep, soils are well- to excessively drained and dry. Species present may include Salix spp., Populus tremuloides, Calamagrostis canadensis, Epilobium angustifolium, Stereocaulon spp., and P. balsamifera trichocarpa.

Steep, south-facing slopes and bluffs on colluvium and older moraine deposits. The tree canopy is dominated by Populus tremuloides. Soils are dry, well to excessively drained, acidic, and rocky with a shallow organic horizon. Understory plants include Picea glauca, Viburnum edule, Rosa acicularis, Linnaea borealis, Epilobium angustifolium, Arctostaphylos uva-ursi, and Shepherdia canadensis.

Steep, south-facing, well-drained bluffs and slopes on colluvium and older moraine deposits with a tree canopy dominated by both Populus tremuloides and Picea glauca. Common understory species include Viburnum edule, Rosa acicularis, Linnaea borealis, Alnus sinuata, Epilobium angustifolium, Arctostaphylos uva-ursi, and Shepherdia canadensis.

Loamy or rocky sites on upper slopes of colluvium or older moraine deposits with herbaceous vegetation. Soils are well-drained and acidic. Vegetation is dominated by Calamagrostis canadensis and Epilobium angustifolium. Other species present may include Equisetum silvaticum, Viburnum edule, Cornus canadensis, and Rosa acicularis. This ecotype can include a sparse cover of trees, but most often was present as inclusions within upland forest or tall shrub classes.

Slopes and crests on colluvium, and older moraine deposits with vegetation dominated by Alnus sinuata. Soils are well-drained, dry to moist, rocky, and acidic, with a shallow layer of organics and loess. Associated plants include Sambucus racemosa, Calamagrostis canadensis, Betula papyrifera, Epilobium angustifolium, Dryopteris dilitata, Rubus idaeus, and Oplopanax horridus.

Slopes and crests on kames, drumlins, older moraine or glaciofluvial deposits with vegetation dominated by Betula papyrifera or less frequently, Populus tremuloides. Soils are moist, well-drained, acidic, and rocky, with a thin layer of organics. Understory plants include Picea glauca, Viburnum edule, Alnus sinuata, Cornus canadensis, Calamagrostis canadensis, Rosa acicularis, Gymnocarpium dryopteris, Lycopodium annotinum, Linnaea borealis, and Pleurozium schreberi.

Slopes and crests on older moraine, kame, and drumlin deposits with a tree canopy dominated by Picea glauca and Betula papyrifera, though Populus tremuloides also may be present. Soils are rocky, welldrained, moist, and acidic with shallow to moderate horizons of organic material and loess. Dominant understory plants include Alnus sinuata, Viburnum edule, Rosa acicularis, Vaccinium vitis-idaea, Linnaea borealis, Cornus canadensis, Calamagrostis canadensis, Gymnocarpium dryopteris, Epilobium angustifolium, and Pleurozium schreberi. This is the most common ecotype on Ft. Richardson.

Upland north- or east-west facing slopes on colluvium or older moraine. Vegetation is dominated by Picea glauca. Soils are moist, well- to somewhat poorly drained, rocky, and acidic, with a moderate to shallow organic horizon. Dominant plants include Betula papyrifera, Alnus sinuata, Viburnum edule, Epilobium angustifolium, Linnaea borealis, Vaccinium uliginosum, Gymnocarpium dryopteris and Hylocomium splendens. Empetrum nigrum differentiaties this class from Upland Rocky Moist Mixed Forest. This ecotype is rare due to heavy damage by fire $\sim 100$ ya and recently by spruce bark beetles.

Flats and depressions on glaciolacustrine, abandoned floodplain, and glaciofluvial channel deposits. Vegetation is dominated by Calamagrostis canadensis. Soils are a mixture of loam and organic material, acidic, and somewhat poorly drained. Associated plants are Epilobium angustifolium, Mertensia paniculata, Betula papyrifera, Cornus canadensis, Picea glauca, Equisetum silvaticum, and E. arvense.

Alluvial fans, lower slopes, and outwash deposits dominated by Alnus sinuata. Soils are moist, gravelly, and somewhat poorly drained. Associated species could include Oplopanax horridus, Heracleum lanatum, Calamagrostis canadensis, and ferns. No ground data were collected for this class. This ecotype occurs in low-lying, flat areas on alluvial plain, old alluvial fans, and glaciofluvial channels with vegetation dominated by Betula papyrifera. Soils are gravelly, well-drained, moist, and acidic with a shallow layer of organics. Understory plants include Picea glauca, Viburnum edule, Rosa acicularis, Calamagrostis canadensis, Vaccinium vitis-idaea, Linnaea borealis, Cornus canadensis, Ribes triste, Trientalis europaea, Lycopodium annotinum, and Pleurozium schreberi. 
Table 9 (cont'd).

Class

Lowland

Gravelly Moist

Mixed Forest

Lowland

Gravelly Moist

Needleleaf

Forest

Lowland Bog

Meadow

Lowland Scrub

Bog

Lowland

Needleleaf

Forest Bog

Lacustrine

Aquatic Forb

Lakes or Ponds

Riverine

Gravelly Moist

Barrens

Riverine

Loamy Wet

Tall Scrub

Riverine

Gravelly Moist

Tall Scrub

Riverine

Gravelly Moist

Broadleaf

Forest

Riverine

Gravelly Moist

Mixed Forest
Low-lying, predominantly flat areas on alluvial plain, old alluvial fans, abandoned floodplains, and glaciofluvial channels and outwash with vegetation dominated by mixed forests. The tree canopy is codominated by Picea glauca and either Populus tremuloides or Betula papyrifera. Soils are gravelly, well-drained, moist, and acidic with a thin layer of organics. Understory plants include Calamagrostis canadensis, Linnaea borealis, Cornus canadensis, Ledum groenlandicum, Vaccinium vitis-idaea, Hylocomium splendens, and Pleurozium schreberi. This is the most commonly occurring lowland ecotype.

Low-lying, predominantly flat areas on alluvial plain, old alluvial fans, abandoned floodplains, and glaciofluvial outwash and channels with a tree canopy dominated by either Picea glauca or Picea mariana. Ledum groenlandicum differentiates this class from upland needleleaf forests when Picea glauca is dominant. Soils are gravelly, well- to moderately well-drained, and acidic with shallow to moderate horizons of organic material and loess. Associated plants include Betula papyrifera, Linnaea borealis, Cornus canadensis, Vaccinium vitis-idaea, Empetrum nigrum, Hylocomium splendens, and Pleurozium schreberi.

Basin and pond margin bogs or fens with deep accumulations of organic material and vegetation is dominated by Sphagnum spp. and sedges. Soils are very poorly drained and strongly acidic. Associated vegetation includes Menyanthes trifoliata, Carex aquatilis, C. rotundata, $C$. rariflora, $C$. lasiocarpa, Andromeda polifolia, Oxycoccus microcarpus, and Betula nana.

Shallow basins and flats on glaciolacustrine and lacustrine deposits with deep accumulations of organic material and vegetation dominated by shrubs. Soils are acidic and poorly to very poorly drained. Two plant associations were merged in this ecotype, one dominated by Betula nana with Rubus chamaemorus, Sphagnum spp. Picea mariana, Ledum palustre decumbens, and Oxycoccus microcarpus; and the other dominated by Myrica gale with Calamagrostis canadensis, Comarum palustre, B. nana, Carex aquatilis, and Sphagnum spp. Both $\mathrm{pH}$ and EC were higher in the second type. Shallow basins and flats on glaciolacustrine and glaciofluvial channel deposits with moderately deep accumulations of organic material and vegetation dominated by Picea mariana and Sphagnum spp. Soils are strongly acidic and poorly to very poorly drained. Associated species include Ledum palustre decumbens, Betula nana, L. groenlandicum, Vaccinium vitis-idaea, V. uliginosum, Empetrum nigrum, Rubus chamaemorus, Equisetum silvaticum, and Pleurozium schreberi.

Shallow ponds $(<1.5 \mathrm{~m})$ or extensive submerged pond margins in basins and depressions with aquatic vegetation. Water is circum-neutral to slightly alkaline. Common plants include Nuphar polysepalum, Menyanthes trifoliata, Equisetum fluviatile, and Potamogeton spp. Merged with Lakes and Ponds for map presentation.

Deep lakes in kettles and basins partially vegetated (5-30\%) with emergent or floating vegetation. Water is acidic and deep $(>1.5 \mathrm{~m})$. Vegetation includes Nuphar polysepalum, Menyanthes trifoliata, Equisetum fluviatile, and Potamogeton spp. Merged with Lacustrine Aquatic Forb for map presentation. Flat gravel bars on active floodplain deposits with vegetation cover less than $30 \%$. Soil is excessively to well-drained, frequently flooded, circum-neutral to slightly alkaline with no organic horizon and few fines. Scattered colonizing species include Populus balsamifera trichocarpa seedlings, Alnus tenuifolia, Epilobium latifolium, Deschampsia caespitosa, Calamagrostis canadensis, and Ceratodon purpureus.

Low-lying areas on active and inactive floodplains and inactive channel deposits with vegetation dominated by Alnus tenuifolia. Soils are loamy, wet, poorly to very poorly drained, and circum-neutral with shallow to moderate organic horizons. Associated plants include Ribes triste, Galium triflorum, Calamagrostis canadensis, Equisetum arvense, Polemonium acutiflorum, and Thalictrum sparsiflorum.

Floodplains of moderately steep headwater streams below treeline with vegetation dominated by tall willows. Soils are well- to moderately well-drained, gravelly, and acidic with shallow to moderately thick organic horizons. Common species include Salix alaxensis, Epilobium angustifolium, Galium triflorum, Equisetum arvense, Alnus sinuata, Calamagrostis canadensis, Heracleum lanatum, and Sanguisorba stipulata.

Inactive floodplains with vegetation dominated by Populus balsamifera trichocarpa. Soils are moist, well-drained, gravelly to sandy, and circum-neutral to acidic, with a thin to moderate surface organic horizon. Buried organic horizons often are present. Understory species include Viburnum edule, Alnus tenuifolia, Picea glauca, Rosa acicularis, Athyrium filix-femina, Heracleum lanatum, Calamagrostis canadensis, Equisetum arvense, and Trientalis europaea.

Inactive floodplains with the tree canopy co-dominated by Picea glauca and either Populus balsamifera trichocarpa or Betula papyrifera. Soils are interbedded sands, silts, and organics, welldrained, and circum-neutral to acidic. Understory plants include Calamagrostis canadensis, Cornus canadensis, Linnaea borealis, Viburnum edule, Rosa acicularis, Mertensia paniculata, and Equisetum arvense. 
Table 9 (cont'd). Classification and description of ecotypes found within Fort Richardson, southcentral Alaska, 2001. Descriptions include physiography, geomorphology, soils properties, and vegetation. Plant names in bold indicate the dominant and differential plants used to define the related plant association.

Class

Riverine

Gravelly Moist

Needleleaf

Forest

Rivers and

Streams

Upper and

Lower

Perennial

Rivers

Coastal Loamy

Wet Barrens

Coastal Loamy

Wet Meadow

(brackish)

Coastal Loamy

Wet Forb

Meadow

(brackish)

Coastal Loamy

Wet Grass

Meadow

(brackish)

Coastal Loamy

Wet Sedge

Meadow

(brackish)

Coastal Sandy

Moist Meadow

Coastal Loamy

Dwarf Scrub

(not mapped)

Coastal Loamy

Wet Meadow

(slightly

brackish)

Coastal Loamy

Wet Low Scrub

Coastal Lake and Marsh

Coastal Marsh

Coastal

Aquatic Forb
Inactive floodplains with vegetation dominated by Picea glauca. Soils are sandy or gravelly, weakly interbedded, with a thin to moderate surface organic horizon, well drained, and acidic. Associated plants include Betula papyrifera, Rosa acicularis, Ledum groenlandicum, Cornus canadensis, Calamagrostis canadensis, Vaccinium vitis-idaea, Linnaea borealis, Equisetum arvense, Hylocomium splendens and Pleurozium schreberi.

A consolidated map class that includes glacial, non-glacial or tidal rivers. Descriptions of individual types are listed under Tidal Rivers and Upper and Lower Perennial Rivers.

Rivers from glacial or non-glacial sources with water flowing year-round in deep channels. Upper Perennial Rivers occur on moderate slopes, have braided floodplains, and are higher energy streams than Lower Perennial Rivers. Lower Perennial Rivers are more slow-moving streams and typically have meander floodplains. Perennial Rivers were grouped for mapping as Rivers and Streams.

Active tidal flats, tidal guts, and channel margins that are unvegetated or partially vegetated $(<30 \%$ cover). Soils are brackish, loamy, and poorly drained with little or no organic matter accumulation. Pioneering plants may include Puccinellia nutkaensis, Triglochin maritimum, Salicornia europaea, and Atriplex gmelini.

Active tidal flats with vegetation dominated by herbaceous species. Soils are saline, wet, loamy, and poorly drained with a shallow surface organic horizon. Coastal Loamy Brackish Wet Meadow is a consolidated map class comprised of Coastal Loamy Wet Forb Meadow (brackish), Coastal Loamy Wet Grass Meadow (brackish), and Coastal Loamy Wet Sedge Meadow (brackish). Descriptions of each follow below.

Active tidal flats, particularly levees, dominated by Triglochin maritimum. Soils are brackish, loamy, and poorly drained. Associated plants include Plantago maritima, Potentilla egedii, Lathyrus palustris, and Puccinellia nutkaensis.

Active tidal flats and basins behind levees, somewhat poorly vegetated and dominated by Puccinellia nutkaensis. Soils are saline, loamy, and poorly drained. Associated plants include Triglochin maritimum, P. phryganodes, Atriplex gmelini, Plantago maritima, and Salicornia europaea.

Upper extents of active tidal flats and basins dominated by nearly pure stands of Carex ramenskii. Soils are saline, loamy, and poorly drained. Other species present include Potentilla egedii, Carex lyngbyaei, and Triglochin maritimum.

Coastal beaches, sandy ridges, and tidal levees dominated by Leymus mollis (Elymus arenarius mollis). Soils are brackish, sandy and well drained. Associated plants include Potentilla egedii, Plantago maritima, and Triglochin maritimum.

Coastal beaches, sandy ridges, and tidal levees dominated by Salix ovalifolia. Soils are brackish, sandy and well drained. Associated plants include Potentilla egedii, Carex lyngbyaei, C. pluriflora, and Leymus mollis.

Inactive tidal flats dominated by nearly pure stands of Carex lyngbyaei. This ecotype is less frequently inundated by tides than those on active tidal flats. Soils are slightly brackish, loamy, and poorly drained. Also included in this class are slightly brackish depressions and shallows populated by $\boldsymbol{C}$. mackenziei and Eleocharis kamtschatica. Associated species for both meadow types are Potentilla egedii, Triglochin maritimum, Carex aquatilis, Scirpus paludosus, and Myriophyllum exalbescens. Inactive tidal flats dominated by Myrica gale. These communities occur at the farthest extent of tidal influence. Soils are slightly brackish, loamy, and poorly drained with thin to moderate organic horizons. Other species present include Calamagrostis canadensis, Carex lyngbyaei, Comarum palustre, and Salix ovalifolia.

Occasionally inundated water bodies with loamy to loamy-organic soils. This consolidated map class includes Coastal Marsh, Coastal Aquatic Forb, and Coastal Ponds. Descriptions of each follow below. Slightly brackish shallow ponds and pond margins on inactive tidal flats with emergent vegetation dominated by Scirpus paludosus. Associated plants include S. validus, Zannichellia palustris, Hippuris tetraphylla, and Ruppia spiralis.

Slightly brackish shallow ponds and pond margins with aquatic herbaceous vegetation. Dominant plants include Zannichellia palustris, Potamogeton pectinatus, and Hippuris tetraphylla. 
Table 9 (cont'd).

\begin{tabular}{|c|c|}
\hline Class & Description \\
\hline Coastal Ponds & $\begin{array}{l}\text { Brackish shallow ponds with less than } 30 \% \text { cover of aquatic vegetation. Tidal inundation varies from } \\
\text { regular to infrequent. }\end{array}$ \\
\hline Tidal River & $\begin{array}{l}\text { Perennial rivers affected by tides. Salinity of the river water is governed by river discharge, tidal stage, } \\
\text { and distance from the coast. Upstream water is fresh at low tide while waters at the river mouth may be } \\
\text { quite saline, particularly at times of low river discharge. }\end{array}$ \\
\hline $\begin{array}{l}\text { Nearshore } \\
\text { Water }\end{array}$ & $\begin{array}{l}\text { Shallow or deep marine waters close to coasts. Nearshore waters are affected by river discharge, run-off } \\
\text { from land surfaces, and land-induced winds and currents. }\end{array}$ \\
\hline $\begin{array}{l}\text { Human } \\
\text { Modified } \\
\text { Barrens }\end{array}$ & $\begin{array}{l}\text { Fill, excavations, or recently modified surfaces that have been altered by human activity and are } \\
\text { unvegetated or partially vegetated }(<30 \% \text { cover }) \text {. Soils and slope position are variable but many sites } \\
\text { in this class have rock or gravel close to the surface and are relatively flat. Recent clearings, airstrips, } \\
\text { paved and gravel roads have been included in this class. Partially vegetated areas may have mixture of } \\
\text { indigenous and introduced species including Agropyron sp., Plantago major, Trifolium spp., } \\
\text { Potentilla spp., and Ceratodon purpureus. }\end{array}$ \\
\hline $\begin{array}{l}\text { Human } \\
\text { Modified } \\
\text { Meadow }\end{array}$ & $\begin{array}{l}\text { Clearings with or without soil removal, roadsides, and areas where vegetation is modified by human } \\
\text { activity. Sites in this class usually have had all natural vegetation removed and are in an early } \\
\text { successional condition. Soils and drainage varies with the degree of soil removal and compaction. } \\
\text { Vegetation includes species found in partially vegetated sites and commercial lawn mixtures along } \\
\text { with Hordeum jubatum, Achillea millifolium, Taraxacum spp., Epilobium angustifolium, Poa spp., } \\
\text { Festuca spp., and Calamagrostis canadensis. }\end{array}$ \\
\hline $\begin{array}{l}\text { Human } \\
\text { Modified } \\
\text { Scrub }\end{array}$ & $\begin{array}{l}\text { Clearings with or without soil removal, roadsides, and areas where vegetation is modified by human } \\
\text { activity. Sites in this class are older or less severely modified than barrens or meadows. Vegetation is } \\
\text { dominated by low or tall shrubs, and tree seedlings and saplings. Species present may include Salix } \\
\text { scouleriana, S. bebbiana, Alnus sinuata, Betula papyrifera, Populus tremuloides, Picea glauca, } \\
\text { Viburnum edule, Ledum groenlandicum, and Rosa acicularis. }\end{array}$ \\
\hline $\begin{array}{l}\text { Human } \\
\text { Modified } \\
\text { Forest }\end{array}$ & $\begin{array}{l}\text { Forests that have been modified by human activity either through selective or clear cutting. These areas } \\
\text { usually are early to mid-successional forest communities similar in composition to undisturbed forest. } \\
\text { Forests in this ecotype were identified based on disturbances evident in old aerial photography. The } \\
\text { canopy usually is dominated by Populus tremuloides or Betula papyrifera with Picea glauca in the } \\
\text { understory. }\end{array}$ \\
\hline $\begin{array}{l}\text { Human } \\
\text { Modified } \\
\text { Waterbody }\end{array}$ & Waterbodies created by human activity, such as water-filled ditches, impoundments, and excavations. \\
\hline $\begin{array}{l}\text { Human } \\
\text { Modified } \\
\text { Complex }\end{array}$ & $\begin{array}{l}\text { Complex assemblage of human-modified land that includes at least three Human Modified ecotype } \\
\text { classes in units too small to map individually. Human Modified Complex was most common in the } \\
\text { cantonment where it typically included roads, buildings, fill, clearings, and landscaping. }\end{array}$ \\
\hline
\end{tabular}

grouped unusual types with more common classes, using relationships identified by analysis of plot data. For example, some lowland forests on abandoned cover deposits had loamy soil, but this class was uncommon and therefore was included in Lowland Gravelly Forest. We also grouped open and closed canopy structures within forest and scrub classes, because vegetation composition generally was similar. The full diversity of ITU combinations, however, is preserved in the database. For special-purpose studies, the ITUs could be recombined to emphasize particular features, such as canopy structure for bird habitat evaluations or geomorphic differences for floodplain evaluation along rivers.

Vegetation characteristics Fort Richardson, with its strong environmental gradients and wide range of habitats, supports a high diversity of plant species. This diversity is reflected in strong differences in growth forms and floristic composition among ecotypes. Approximately 340 vascular and 150 non-vascular species were identified during this study. A more complete list of 561 vascular and 239 non-vascular species was developed by Lichvar et al. (1997) and is summarized in Appendixes 4 and 5. In the following discussion, we highlight some of the differences and similarities in growth forms (Fig. 19) and species composition (Tables 11-15) among the ecotypes, focusing primarily on identifying the species that dominate the various ecotypes and that can be used to help differentiate classes. To facilitate comparisons, ecotypes were grouped by physiography and vegetation structure.

Alpine ecotypes were dominated by dwarf and low shrubs, and also had a substantial moss and lichen component (Fig. 19). Species that were common in nearly all alpine ecotypes included Dryas octopetala, 


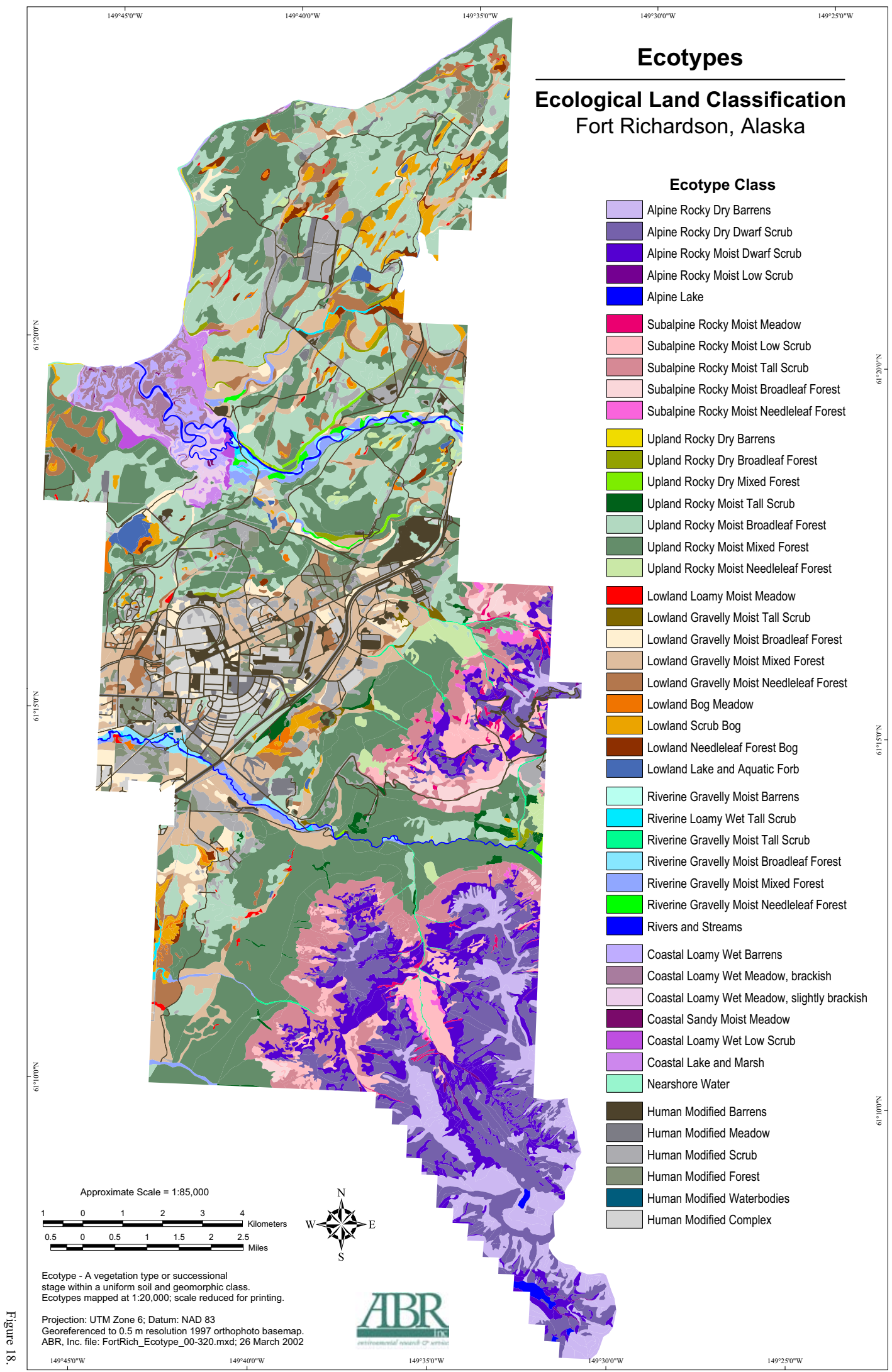

Figure 18. Map of ecotypes on Fort Richardson, south-central Alaska, 2001. 
Table 10. Areal extent of ecotypes mapped on Fort Richardson, south-central Alaska, 2001.

\begin{tabular}{|c|c|c|c|}
\hline \multirow[b]{2}{*}{ Ecotype } & \multicolumn{3}{|c|}{ Area } \\
\hline & acre & ha & $\%$ \\
\hline Alpine Rocky Dry Barrens & 2683 & 1086 & 4.3 \\
\hline Alpine Rocky Dry Dwarf Scrub & 4946 & 2002 & 8.0 \\
\hline Alpine Rocky Moist Dwarf Scrub & 3386 & 1370 & 5.5 \\
\hline Alpine Rocky Moist Low Scrub & 92 & 37 & 0.1 \\
\hline Alpine Lake & 91 & 37 & 0.1 \\
\hline Subalpine Rocky Moist Meadow & 190 & 77 & 0.3 \\
\hline Subalpine Rocky Moist Low Scrub & 1575 & 638 & 2.5 \\
\hline Subalpine Rocky Moist Tall Scrub & 2788 & 1128 & 4.5 \\
\hline Subalpine Rocky Moist Broadleaf Forest & 543 & 220 & 0.9 \\
\hline Subalpine Rocky Moist Needleleaf Forest & 176 & 71 & 0.3 \\
\hline Upland Rocky Dry Barrens & 39 & 16 & 0.1 \\
\hline Upland Rocky Dry Broadleaf Forest & 197 & 80 & 0.3 \\
\hline Upland Rocky Dry Mixed Forest & 102 & 41 & 0.2 \\
\hline Upland Rocky Moist Tall Scrub & 314 & 127 & 0.5 \\
\hline Upland Rocky Moist Broadleaf Forest & 9569 & 3872 & 15.4 \\
\hline Upland Rocky Moist Mixed Forest & 13,762 & 5569 & 22.2 \\
\hline Upland Rocky Moist Needleleaf Forest & 709 & 287 & 1.1 \\
\hline Lowland Loamy Moist Meadow & 69 & 28 & 0.1 \\
\hline Lowland Gravelly Moist Tall Scrub & 73 & 30 & 0.1 \\
\hline Lowland Gravelly Moist Broadleaf Forest & 1131 & 458 & 1.8 \\
\hline Lowland Gravelly Moist Mixed Forest & 4447 & 1800 & 7.2 \\
\hline Lowland Gravelly Moist Needleleaf Forest & 1887 & 764 & 3.0 \\
\hline Lowland Bog Meadow & 190 & 77 & 0.3 \\
\hline Lowland Scrub Bog & 1003 & 406 & 1.6 \\
\hline Lowland Needleleaf Forest Bog & 308 & 125 & 0.5 \\
\hline Lowland Lake and Aquatic Forb & 235 & 95 & 0.4 \\
\hline Riverine Gravelly Moist Barrens & 29 & 12 & $<0.1$ \\
\hline Riverine Loamy Wet Tall Scrub & 87 & 35 & 0.1 \\
\hline Riverine Gravelly Moist Tall Scrub & 137 & 56 & 0.2 \\
\hline Riverine Gravelly Moist Broadleaf Forest & 279 & 113 & 0.4 \\
\hline Riverine Gravelly Moist Mixed Forest & 503 & 204 & 0.8 \\
\hline Riverine Gravelly Moist Needleleaf Forest & 108 & 44 & 0.2 \\
\hline Rivers and Streams & 169 & 68 & 0.3 \\
\hline Coastal Loamy Wet Barrens & 689 & 279 & 1.1 \\
\hline Coastal Loamy Wet Meadow, brackish & 542 & 220 & 0.9 \\
\hline Coastal Loamy Wet Meadow, slightly brackish & 359 & 145 & 0.6 \\
\hline Coastal Sandy Moist Meadow & 16 & 6 & $<0.1$ \\
\hline Coastal Loamy Wet Low Scrub & 153 & 62 & 0.2 \\
\hline Coastal Lake and Marsh & 421 & 170 & 0.7 \\
\hline Nearshore Water & 26 & 11 & $<0.1$ \\
\hline Human Modified Barrens & 1614 & 653 & 2.6 \\
\hline Human Modified Meadow & 667 & 270 & 1.1 \\
\hline Human Modified Scrub & 3288 & 1331 & 5.3 \\
\hline Human Modified Forest & 1121 & 454 & 1.8 \\
\hline Human Modified Waterbodies & 9 & 3 & $<0.1$ \\
\hline Human Modified Complex & 1272 & 515 & 2.1 \\
\hline Total & 61,996 & 25,089 & 100 \\
\hline
\end{tabular}




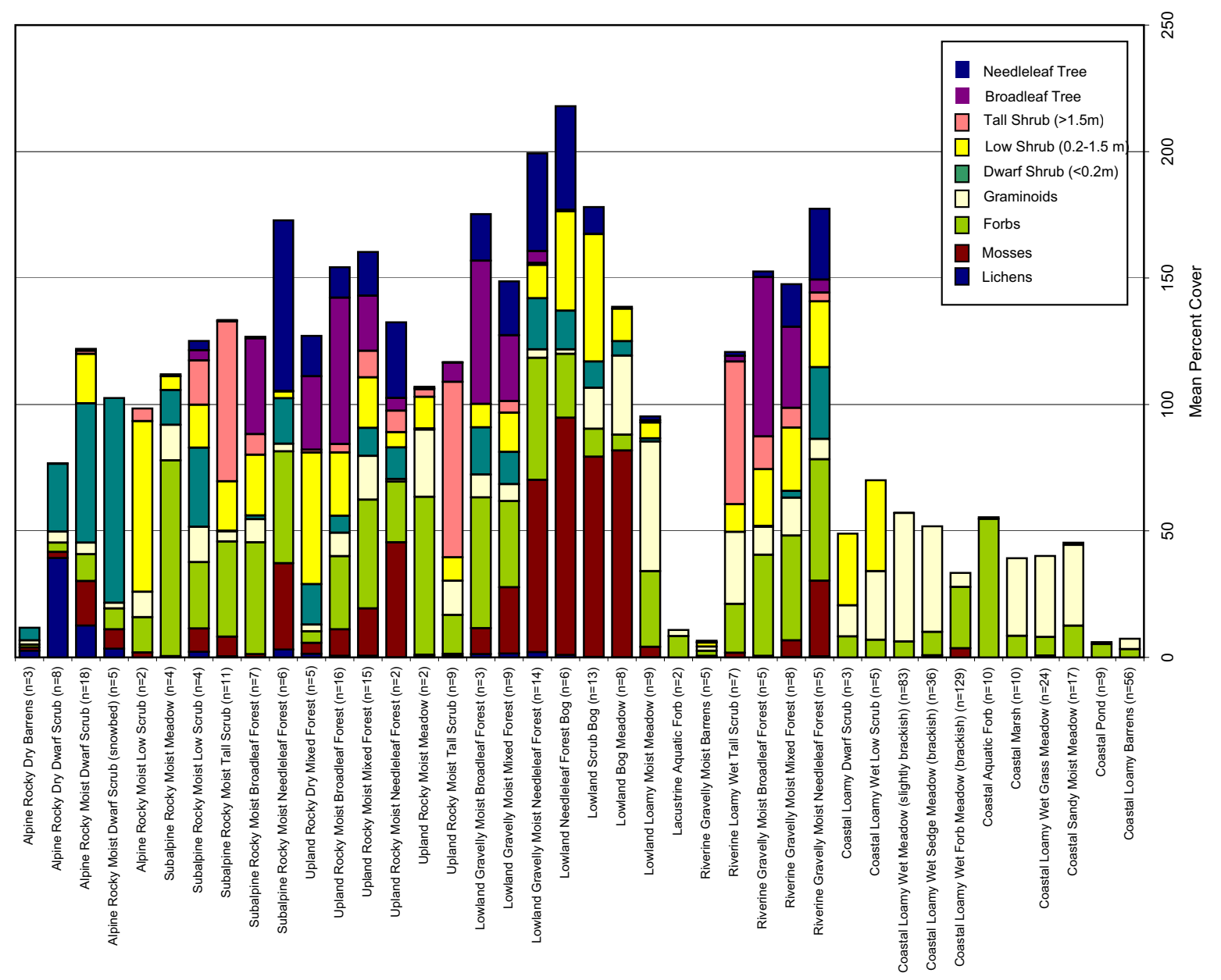

Figure 19. Percent cover of growth forms by ecotype on Fort Richardson, south-central Alaska, 2001.

Vaccinium vitis-idaea, Hierochloë alpine, Salix arctica, Empetrum nigrum, Salix planifolia pulchra, Thamnolia spp., Cetraria nivalis, and Cetraria islandica (Table 11). There was strong floristic zonation, however, with large differences in total composition among Alpine Rocky Dry Barrens in high, exposed environments, Alpine Rocky Moist Dwarf Scrub (dominated by Empetrum nigrum and differentiated by the frequent occurrence of Betula nana) on more protected slopes, and Alpine Rocky Moist Low Scrub (dominated by Salix planifolia and differentiated by Sanguisorba stipulata) in drainages (Table 11, Fig. 20). In contrast, there was moderate floristic similarity between Alpine Rocky Dry Barrens and Alpine Rocky Dry Dwarf Scrub (dominated by Dryas octopetala and differentiated by frequent occurrence of Hierochloë alpina), and between Alpine Rocky Moist Dwarf Scrub-Snowbed (dominated by Cassiope stelleriana and differentiated by Luetkea pectinata) and Alpine Rocky Moist Dwarf Scrub. These latter ecotypes were readily distinguished, however, as a result of strong differences in the dominant species (Fig. 20). These ecotypes and their associated plant communities were similar to those described by Walker et al. (1997), but several of the classes were subdivided in their analysis.

Subalpine ecotypes, which occur in a transition zone from upland to alpine ecosystems, included growth forms ranging from lush forb meadows to dwarf needleleaf trees (Fig. 19). There was little floristic similarity between subalpine and alpine ecotypes, except that Subalpine Rocky Low Scrub overlapped substantially with Alpine Rocky Dwarf Scrub. Species that were common in the subalpine zone, but generally absent elsewhere, included Tsuga mertensiana, Valeriana sitchensis, Veratrum viride, Salix barclayi, Geranium erianthum, and Aconitum delphinifolium (Table 11). Strong differences in relative abundances of species resulted in distinct associations (Fig. 20). Subalpine Rocky Moist Low Scrub was dominated by Betula nana and Salix scouleriana, but no reliable differential spe- 
Table 11. Mean cover (\%) of the most abundant species within alpine and subalpine ecotypes on Fort Richardson, south-central Alaska, 2001. Bold numbers represent frequencies $\geq 60 \%$ within ecotype, blank when absent, and $0=<0.5 \%$ cover.

\begin{tabular}{|c|c|c|c|c|c|c|c|c|c|c|}
\hline Taxon & 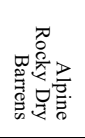 & 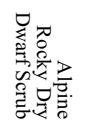 & 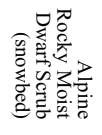 & 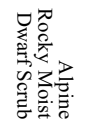 & 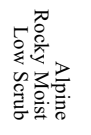 & 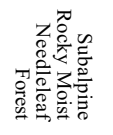 & 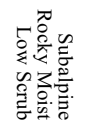 & 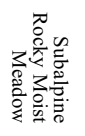 & 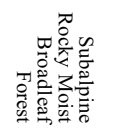 & 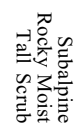 \\
\hline Saxifraga bronchialis & 0 & 0 & & 0 & & & & & & \\
\hline Thamnolia sp. & 0 & 2 & 0 & 0 & & & & & & \\
\hline Sphaerophorus globosus & & 0 & & 0 & & & & & & \\
\hline Carex microchaeta & & 1 & 0 & & & & & & & \\
\hline Oxytropis bryophila & & 1 & & 0 & & & & & & \\
\hline Alectoria ochroleuca & & 5 & & 1 & & & & & & \\
\hline Bryocaulon divergens & & 3 & & 0 & & & & & & \\
\hline Cetraria nivalis & 1 & 6 & 0 & 2 & & & & & & \\
\hline Diapensia lapponica & & 2 & 0 & 0 & & & & & & \\
\hline Dryas octopetala & 3 & 19 & & 2 & & & & & & \\
\hline Hierochloe alpina & 0 & 2 & 0 & 0 & & & & & & \\
\hline Salix arctica & 1 & 3 & 0 & 1 & & & & & & \\
\hline Cetraria cucullata & 0 & 2 & & 1 & & & & & & \\
\hline Gentiana glauca & & 0 & 0 & 0 & & & & & & \\
\hline Cladina arbuscula & & 4 & 0 & 0 & & 0 & & & & \\
\hline Arctous alpina & & 1 & & 6 & & & & & & \\
\hline Cladina sp. & & 3 & 1 & 2 & & 0 & 1 & & & \\
\hline Stereocaulon sp. & 1 & 2 & 0 & 1 & & & 1 & & & \\
\hline Cetraria islandica & 0 & 0 & 0 & 0 & & 0 & & & & \\
\hline Cladina stellaris & & 6 & 1 & 1 & & & 1 & & & \\
\hline Cladonia sp. & 1 & 0 & 0 & 0 & & 0 & 0 & & 0 & \\
\hline Cassiope stelleriana & & & 43 & 0 & & & & & & \\
\hline Luetkea pectinata & & & 25 & 1 & & 0 & & & & \\
\hline Lycopodium alpinum & & & 5 & 0 & & & & 0 & & \\
\hline Huperzia selago & & & 1 & & & & & 0 & & \\
\hline Hylocomium splendens & & 0 & & 2 & & 6 & & & & \\
\hline Vaccinium vitis-idaea & 0 & 1 & 0 & 3 & & 1 & 3 & 1 & 0 & \\
\hline Ledum palustre decumbens & & 0 & 0 & 2 & & 0 & 0 & & & \\
\hline Empetrum nigrum & & 2 & 19 & 41 & 1 & 14 & 10 & 10 & & 0 \\
\hline Betula nana & & 0 & 0 & 20 & & 0 & 11 & 0 & 0 & 3 \\
\hline Pleurozium schreberi & & & 1 & 3 & & 9 & 13 & & & \\
\hline Betula occidentalis & & & & 2 & & 0 & 5 & & & \\
\hline Vaccinium uliginosum & & 1 & 0 & 10 & & 0 & 7 & 5 & & 1 \\
\hline Salix planifolia pulchra & & 0 & 1 & 1 & 53 & 0 & & & 0 & 0 \\
\hline Salix glauca & & & & 0 & & & 3 & & & \\
\hline Sanguisorba stipulata & & & 0 & 0 & 3 & 1 & 4 & 10 & 4 & 0 \\
\hline Castilleja unalaschcensis & & & 0 & 0 & & & 1 & 0 & 1 & 0 \\
\hline Artemisia arctica & 0 & 0 & 0 & 1 & & 0 & 3 & 0 & 0 & \\
\hline Festuca altaica & & 0 & 1 & 1 & & 0 & 9 & 4 & 0 & \\
\hline Tsuga mertensiana & & 0 & 0 & 0 & & 68 & & & & \\
\hline Cornus canadensis & & & & 8 & 1 & 19 & 10 & 7 & 19 & 3 \\
\hline Linnaea borealis & & & & 1 & & 4 & 1 & 1 & 2 & 0 \\
\hline Lycopodium annotinum & & & 0 & 0 & 1 & 2 & 1 & & 0 & 1 \\
\hline Spiraea beauverdiana & & & 0 & 0 & 1 & 1 & & 0 & & 1 \\
\hline Salix scouleriana & & & & 1 & 1 & 0 & 18 & & 4 & 7 \\
\hline Gymnocarpium dryopteris & & & & 0 & & 14 & 4 & 2 & 8 & 7 \\
\hline Artemisia tilesii & & & 0 & 0 & & & & 5 & 0 & 0 \\
\hline Veratrum viride & & & 0 & 0 & 1 & 0 & 0 & 10 & 8 & 1 \\
\hline Rubus pedatus & & & 0 & 0 & & 4 & 0 & & & 2 \\
\hline Calamagrostis canadensis & & & & 1 & 5 & 3 & 8 & 7 & 4 & 3 \\
\hline Rubus arcticus & & & & 0 & 1 & 0 & 1 & 1 & 0 & 0 \\
\hline Epilobium angustifolium & & & & 0 & 10 & 1 & 9 & 16 & 10 & 5 \\
\hline Geranium erianthum & & & & 0 & & 1 & 6 & 9 & 4 & 0 \\
\hline Salix barclayi & & & & 1 & 10 & 0 & 1 & 3 & & 1 \\
\hline Trientalis europaea & & & & 0 & 0 & 1 & 1 & 0 & 0 & 0 \\
\hline Equisetum arvense & & & & 3 & & & & 3 & 0 & 0 \\
\hline Rhizocarpon sp. & & & & 0 & 0 & 2 & & & & 0 \\
\hline Valeriana sitchensis & & & & 0 & & 1 & 0 & 19 & 2 & 3 \\
\hline Salix bebbiana & & & & 0 & & & 5 & 0 & 2 & 3 \\
\hline Brachythecium sp. & & & & 0 & 1 & 4 & 2 & & 0 & 1 \\
\hline Streptopus amplexifolius & & & & & & 1 & & 0 & 0 & 0 \\
\hline Picea glauca & & & & 1 & & & 5 & 0 & 1 & \\
\hline Thelypteris phegopteris & & & & & & 0 & & 4 & 2 & 0 \\
\hline Lupinus nootkatensis & & & & 0 & & 0 & 2 & & 2 & \\
\hline Sorbus scopulina & & & & 0 & & 1 & & 1 & 2 & 0 \\
\hline Achillea millefolium & & & & 0 & 1 & & 1 & 2 & 1 & 0 \\
\hline Pyrola asarifolia & & & 0 & & & 0 & 0 & 0 & 3 & 0 \\
\hline Rosa acicularis & & & & & & 0 & 3 & 1 & 3 & \\
\hline Populus balsamifera balsamifera & & & & & & & 0 & & 41 & 1 \\
\hline
\end{tabular}


Table 11 (cont'd). Mean cover (\%) of the most abundant species within alpine and subalpine ecotypes on Fort Richardson, south-central Alaska, 2001. Bold numbers represent frequencies $\geq 60 \%$ within ecotype, blank when absent, and $0=<0.5 \%$ cover.

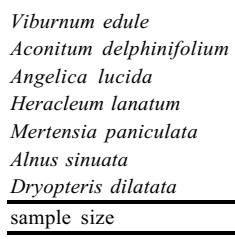

cies was evident for this ecotype. Subalpine Rocky Moist Meadow was distinguished by high cover of forbs, especially Valeriana sitchensis, Sanguisorba stipulata, and Geranium erianthum although a reliable differential species was not present. Subalpine Rocky Moist Tall Shrub was dominated by Alnus sinuata and differentiated from Upland Rocky Moist Tall Shrub by the presence of Streptopus amplexifolius. Subalpine Rocky Moist Needleleaf Forest was dominated by an open to closed cover of dwarf Tsuga mertensiana, which was sufficient to differentiate this class from all other ecotypes. Finally, Subalpine Rocky Moist Broadleaf Forest was dominated by an open canopy of Populus balsamifera balsamifera, and was differentiated from Riverine Gravelly Moist Broadleaf Forests by the common occurrence of Aconitum delphinifolium.

Forest ecotypes on rocky uplands and gravelly lowlands included both broadleaf and needleleaf forests; understories usually were dominated by forbs and low shrubs (Fig. 19). All these forest ecotypes were floristically similar (Table 12, Fig. 21). Picea glauca and Betula papyrifera were the most abundant trees in nearly all forest types, while understory species that were found in nearly every forest ecotype included Ribes triste, Viburnum edule, Rosa acicularis, Vaccinium vitis-idaea, Calamagrostis canadensis, Epilobium angustifolium, Linnaea borealis, Cornus canadensis, Geocaulon lividum, and Pleurozium schreberi. Some minor differences were evident; Alnus sinuata, Sorbus scopulina, and Viburnum edule were more abundant in upland forests, while lowland forests included more Picea mariana, Vaccinium vitis-idaea, Ledum groenlandicum, and feather mosses.

Despite these overall similarities, we were able to differentiate the forest ecotypes based on the dominant tree species and, to a lesser extent, on the understory species (Table 12). Upland Rocky Dry Mixed Forest was the most distinct forest ecotype, and was differentiated by the dominance of Populus tremuloides and the frequent presence of Shepherdia canadensis and Arctostaphylos uva-ursi. Upland Rocky Moist Broadleaf Forest and Lowland Gravelly Moist Broadleaf For- est were assigned a single plant association that was dominated by Betula papyrifera and differentiated by the presence of Lycopodium annotinum. Upland Rocky Moist Mixed Forest was dominated by Betula papyrifera and Picea glauca and differentiated from Lowland Gravelly Moist Mixed Forest by the presence of Alnus sinuata and the lack of Ledum groenlandicum. The two plant associations for Lowland Gravelly Moist Mixed Forest (Table 8) were based primarily on differences in the relative dominance of Betula papyrifera and Populus tremuloides in the overstory. Upland Rocky Moist Needleleaf Forest was floristically similar to Upland Rocky Moist Mixed Forest and was assigned the same plant association (Table 8). We were unable to differentiate the upland mixed and needleleaf forests, largely because of the low occurrence of Upland Rocky Moist Needleleaf Forest on Fort Richardson. Pure stands of Picea glauca have never been common in the area, and those that did exist have been decimated by the spruce bark beetle. Finally, Lowland Gravelly Moist Needleleaf Forest was dominated by Picea glauca and Picea mariana. It was differentiated from Upland Rocky Moist Needleleaf Forest by the frequent occurrence of Ledum groenlandicum and the lack of Alnus sinuata and was differentiated from mixed forest by lack of broadleaf trees and the presence of Empetrum nigrum.

Two non-forested ecotypes also occurred in upland situations. Upland Rocky Moist Tall Scrub was dominated by tall shrubs, and differentiated by the strong dominance of Alnus sinuata in the open to closed canopy (Fig. 19). Upland Rocky Moist Meadow was dominated by graminoids and forbs, and was differentiated by the dominance of Calamagrostis canadensis and Epilobium angustifolium, and lack of Alnus. These types were floristically similar, and differed mainly in the relative abundances of dominant species. Upland meadows were documented in ground data but were not mapped because they usually occurred as inclusions within tall scrub or forest ecotypes. Upland Rocky Moist Meadow also was floristically similar to Lowland Loamy Moist Meadow; these types were differentiated primarily on 
Table 12. Mean cover (\%) of the most abundant species within upand and lowland forest ecotypes on Fort Richardson, south-central Alaska, 2001. Bold numbers represent frequencies $\geq 60 \%$ within ecotype, blank when absent, and $0=<0.5 \%$ cover.

\begin{tabular}{|c|c|c|c|c|c|c|c|}
\hline Taxon & $\begin{array}{l}\text { Upland } \\
\text { Rocky Dry } \\
\text { Mixed } \\
\text { Forest }\end{array}$ & $\begin{array}{l}\text { Lowland } \\
\text { Gravelly Moist } \\
\text { Broadleaf } \\
\text { Forest }\end{array}$ & $\begin{array}{l}\text { Upland Rocky } \\
\text { Moist } \\
\text { Broadleaf } \\
\text { Forest }\end{array}$ & $\begin{array}{c}\text { Upland } \\
\text { Rocky } \\
\text { Moist } \\
\text { Mixed } \\
\text { Forest }\end{array}$ & $\begin{array}{c}\text { Lowland } \\
\text { Gravelly Moist } \\
\text { Mixed Forest }\end{array}$ & $\begin{array}{l}\text { Upland Rocky } \\
\text { Moist } \\
\text { Needleleaf } \\
\text { Forest }\end{array}$ & $\begin{array}{c}\text { y Lowland } \\
\text { Gravelly Moist } \\
\text { Needleleaf } \\
\text { Forest }\end{array}$ \\
\hline Arctostaphylos uva-ursi & 8 & & & & & & \\
\hline Shepherdia canadensis & 17 & & 0 & 2 & & & 0 \\
\hline Actaea rubra & 0 & & 0 & 0 & 0 & & \\
\hline Galium triflorum & 0 & 0 & 0 & 0 & 0 & & \\
\hline Oplopanax horridus & 1 & & 2 & 1 & 5 & & \\
\hline Sambucus racemosa & 0 & & 0 & 0 & 1 & 1 & 0 \\
\hline Sorbus scopulina & 0 & & 0 & 1 & & 3 & \\
\hline Moehringia lateriflora & 0 & 1 & & 0 & 0 & & 0 \\
\hline Pyrola chlorantha & 0 & $\mathbf{0}$ & 0 & & 0 & & \\
\hline Mertensia paniculata & 1 & 2 & 0 & 0 & 1 & 0 & 0 \\
\hline Populus balsamifera trichocarpa & 2 & & & 2 & 0 & 3 & \\
\hline Dryopteris dilatata & & $\mathbf{0}$ & 0 & 1 & 1 & & 0 \\
\hline Rubus idaeus & 0 & & 0 & 1 & 0 & & 1 \\
\hline Populus tremuloides & 25 & & 7 & 3 & 17 & & 1 \\
\hline Alnus sinuata & 5 & & 3 & 9 & 2 & 1 & 0 \\
\hline Cladonia sp. & $\mathbf{0}$ & 0 & 0 & 0 & & $\mathbf{0}$ & 0 \\
\hline Lycopodium annotinum & 4 & 1 & 4 & 1 & 1 & 0 & \\
\hline Ribes triste & 1 & 1 & 0 & 0 & 1 & & 0 \\
\hline Trientalis europaea & 0 & 1 & 0 & 0 & 0 & & 0 \\
\hline Viburnum edule & 31 & 7 & 18 & 12 & 0 & 3 & \\
\hline Rosa acicularis & 8 & 2 & 1 & 6 & 0 & & 2 \\
\hline Betula papyrifera & 4 & 57 & 50 & 15 & 10 & 3 & 4 \\
\hline Calamagrostis canadensis & 1 & 6 & 8 & 16 & 5 & 1 & 3 \\
\hline Epilobium angustifolium & $\mathbf{0}$ & 2 & 1 & 6 & 2 & 2 & 1 \\
\hline Picea glauca & 16 & 18 & 11 & 17 & 21 & 30 & 20 \\
\hline Linnaea borealis & 5 & 7 & 7 & 4 & 6 & 3 & 6 \\
\hline Cornus canadensis & 0 & 45 & 16 & 28 & 19 & 2 & 38 \\
\hline Vaccinium vitis-idaea & 1 & 19 & 3 & 5 & 8 & 3 & 12 \\
\hline Peltigera canina & 1 & 0 & 0 & 0 & 0 & 0 & 0 \\
\hline Geocaulon lividum & 1 & 1 & 0 & 0 & 3 & 3 & 1 \\
\hline Peltigera aphthosa & 0 & 1 & 0 & 0 & 2 & 1 & 2 \\
\hline Hylocomium splendens & 0 & 2 & 1 & 6 & 8 & 43 & 14 \\
\hline Equisetum arvense & & 2 & 1 & 0 & 3 & & 1 \\
\hline Gymnocarpium dryopteris & & 12 & 10 & 11 & 4 & 8 & 0 \\
\hline Orthilia secunda & & 1 & 0 & 0 & & 1 & 0 \\
\hline Dicranum sp. & 0 & 0 & 0 & 0 & 0 & $\mathbf{0}$ & 0 \\
\hline Brachythecium sp. & 1 & 0 & 0 & 1 & 1 & & 2 \\
\hline Salix bebbiana & & 0 & 0 & 0 & 2 & 3 & 1 \\
\hline Salix scouleriana & 0 & & 0 & & & & 0 \\
\hline Achillea millefolium & 0 & & 0 & 0 & & $\mathbf{0}$ & 0 \\
\hline Polytrichum sp. & & 5 & 1 & 0 & 0 & 0 & 0 \\
\hline Pleurozium schreberi & & 8 & 8 & 11 & 18 & 3 & 48 \\
\hline Calamagrostis inexpansa & & 2 & 0 & 0 & & & 0 \\
\hline Geranium erianthum & & & & 0 & & 3 & \\
\hline Lupinus nootkatensis & & & 0 & 0 & 1 & 1 & 1 \\
\hline Drepanocladus sp. & & 1 & 0 & 0 & 0 & $\mathbf{0}$ & 0 \\
\hline Menziesia ferruginea & & & 0 & 3 & 1 & & \\
\hline Lycopodium complanatum & & 0 & 0 & 0 & 4 & & 2 \\
\hline Ptilium crista-castrensis & & & 0 & 1 & 1 & & 1 \\
\hline Ledum groenlandicum & & 0 & 4 & 1 & 11 & & 9 \\
\hline Betula nana & & & & 2 & & & 0 \\
\hline Equisetum silvaticum & & 1 & 0 & 5 & 0 & & 5 \\
\hline Empetrum nigrum & & & & 1 & 1 & 3 & 4 \\
\hline Picea mariana & & & 0 & 1 & & & 20 \\
\hline Vaccinium uliginosum & & & & 0 & & 8 & 1 \\
\hline Spiraea beauverdiana & & 0 & & 0 & & & 0 \\
\hline Salix glauca & & & & & 0 & 3 & \\
\hline Salix planifolia pulchra & & & & & & 3 & \\
\hline sample size & 5 & 3 & 16 & 15 & 9 & 2 & 14 \\
\hline
\end{tabular}


Table 13. Mean cover (\%) of the most abundant species within upland and lowland scrub, and lacustrine ecotypes on Fort Richardson, south-central Alaska, 2001. Bold numbers represent frequencies $\geq 60 \%$ within ecotype, blank when absent, and $0=<0.5 \%$ cover.

\begin{tabular}{|c|c|c|c|c|c|c|c|c|}
\hline Taxon & $\begin{array}{l}\text { Lakes or } \\
\text { Ponds }\end{array}$ & $\begin{array}{l}\text { Lacustrine } \\
\text { Aquatic } \\
\text { Forb }\end{array}$ & $\begin{array}{c}\text { Lowland Bog } \\
\text { Meadow }\end{array}$ & $\begin{array}{l}\text { Lowland } \\
\text { Scrub Bog }\end{array}$ & $\begin{array}{c}\text { Lowland } \\
\text { Needleleaf Forest } \\
\text { Bog }\end{array}$ & $\begin{array}{c}\text { Lowland } \\
\text { Loamy Moist } \\
\text { Meadow }\end{array}$ & $\begin{array}{l}\text { Upland Rocky } \\
\text { Moist Meadow }\end{array}$ & $\begin{array}{c}\text { Upland Rocky } \\
\text { Moist Tall } \\
\text { Scrub }\end{array}$ \\
\hline Potamogeton epihydrus & 0 & & & & & & & \\
\hline Menyanthes trifoliata & 2 & 1 & 3 & & 1 & & & \\
\hline Nuphar polysepalum & 4 & 7 & & & & & & \\
\hline Potamogeton sp. & $\mathbf{0}$ & 3 & & & & & & \\
\hline Potamogeton gramineus & & 3 & & & & & & \\
\hline Equisetum fluviatile & 0 & 1 & 1 & 0 & & & & \\
\hline Comarum palustre & & 0 & 1 & 4 & & 0 & & \\
\hline Equisetum palustre & & 0 & 0 & 0 & & & & \\
\hline Sphagnum riparium & & & 12 & & & & & \\
\hline Carex limosa & & & 4 & & & & & \\
\hline Scorpidium scorpioides & & & 3 & & & & & \\
\hline Eriophorum scheuchzeri & & & 2 & 0 & & & & \\
\hline Carex aquatilis & & & 6 & 4 & & & & \\
\hline Carex membranacea & & & 2 & 1 & & & & \\
\hline Carex pauciflora & & & 2 & 0 & & & & \\
\hline Carex rariflora & & & 5 & 0 & & & & \\
\hline Carex rotundata & & & 4 & 0 & & & & \\
\hline Carex lasiocarpa & & & 3 & 0 & & & & \\
\hline Sphagnum fuscum & & & 3 & 4 & & & & \\
\hline Drosera rotundifolia & & & 1 & 0 & & & & \\
\hline Myrica gale & & & 3 & 17 & & & & \\
\hline Spiranthes romanzoffiana & & & 0 & 0 & & & & \\
\hline Oxycoccus microcarpus & & & 3 & 3 & 1 & & & \\
\hline Chamaedaphne calyculata & & & 1 & 2 & 1 & 0 & & \\
\hline Empetrum nigrum & & & 1 & 2 & 5 & & & \\
\hline Andromeda polifolia & & & 4 & 2 & 0 & 0 & & \\
\hline Ledum palustre decumbens & & & 1 & 6 & 2 & & & \\
\hline Picea mariana & & & 1 & 11 & 39 & 1 & & \\
\hline Sphagnum sp. & & & 44 & 54 & 54 & 1 & & \\
\hline Betula nana & & & 6 & 17 & 3 & 1 & & \\
\hline Eriophorum angustifolium & & & 3 & 1 & 0 & & & \\
\hline Salix fuscescens & & & 2 & 3 & 0 & 0 & & \\
\hline Salix planifolia pulchra & & & 0 & 2 & 0 & & & \\
\hline Ledum groenlandicum & & & 0 & 6 & 24 & 1 & & 0 \\
\hline Rubus chamaemorus & & & 1 & 7 & 10 & 2 & & \\
\hline Tomentypnum nitens & & & & 5 & & & & \\
\hline Pleurozium schreberi & & & & 1 & 18 & & & \\
\hline Hylocomium splendens & & & & 2 & 7 & & & \\
\hline Peltigera aphthosa & & & & 0 & 1 & & & \\
\hline Sphagnum girgensohnii & & & & & 12 & & & \\
\hline Vaccinium uliginosum & & & & 2 & 6 & 0 & & \\
\hline Vaccinium vitis-idaea & & & & 2 & 13 & 0 & & \\
\hline Pentaphylloides floribunda & & & & 2 & 0 & 1 & & \\
\hline Betula papyrifera & & & 0 & 0 & 1 & 1 & 4 & 8 \\
\hline Calamagrostis canadensis & & & 0 & 12 & 1 & 51 & 32 & 14 \\
\hline Equisetum arvense & & & & 1 & 3 & 3 & & 3 \\
\hline Equisetum sylvaticum & & & & 0 & 23 & 4 & 8 & 0 \\
\hline Cornus canadensis & & & & & 1 & 1 & 13 & 0 \\
\hline Spiraea beauverdiana & & & & 1 & 2 & 1 & & 0 \\
\hline Stellaria sp. & & & & 0 & & 0 & 0 & 0 \\
\hline Salix scouleriana & & & & & 2 & & & 1 \\
\hline Salix bebbiana & & & & & 0 & 0 & & 3 \\
\hline Drepanocladus sp. & & & & & 3 & 0 & & \\
\hline Epilobium angustifolium & & & & & & 12 & 45 & 6 \\
\hline Heracleum lanatum & & & & & & 2 & 2 & \\
\hline Mertensia paniculata & & & & & & 5 & 3 & 1 \\
\hline Rosa acicularis & & & & 0 & & 1 & 3 & 0 \\
\hline Gymnocarpium dryopteris & & & & & & 0 & 5 & 0 \\
\hline Picea glauca & & & & 0 & & 2 & 0 & 0 \\
\hline Rubus idaeus & & & & 0 & & 2 & & 1 \\
\hline Viburnum edule & & & & & & 0 & 15 & 1 \\
\hline Trientalis europaea & & & & 0 & & 1 & & 0 \\
\hline Alnus sinuata & & & & & & & 1 & 58 \\
\hline Dryopteris dilatata & & & & & & & 0 & 8 \\
\hline Oplopanax horridus & & & & & & & & 2 \\
\hline Ribes triste & & & & & & & & 2 \\
\hline Sambucus racemosa & & & & & & & & 5 \\
\hline sample size & 3 & 2 & 8 & 13 & 6 & 9 & 2 & 9 \\
\hline
\end{tabular}


Table 14. Mean cover (\%) of the most abundant species within riverine ecotypes on Fort Richardson, south-central Alaska, 2001. Bold numbers represent frequencies $\geq 60 \%$ within ecotype, blank when absent, and $0=<0.5 \%$ cover.

\begin{tabular}{|c|c|c|c|c|c|c|}
\hline Taxon & $\begin{array}{c}\text { Riverine } \\
\text { Gravelly Moist } \\
\text { Needleleaf } \\
\text { Forest }\end{array}$ & $\begin{array}{c}\text { Riverine } \\
\text { Gravelly Moist } \\
\text { Mixed Forest }\end{array}$ & $\begin{array}{c}\text { Riverine } \\
\text { Gravelly Moist } \\
\text { Broadleaf Forest }\end{array}$ & $\begin{array}{c}\text { Riverine } \\
\text { Gravelly } \\
\text { Moist Tall } \\
\text { Scrub }\end{array}$ & $\begin{array}{l}\text { Riverine } \\
\text { Loamy Wet } \\
\text { Tall Scrub }\end{array}$ & $\begin{array}{c}\text { Riverine } \\
\text { Gravelly } \\
\text { Moist Barrens }\end{array}$ \\
\hline Picea mariana & 2 & & & & 1 & \\
\hline Ledum groenlandicum & 3 & & & & & \\
\hline Ptilium crista-castrensis & 0 & 0 & & & & \\
\hline Pleurozium schreberi & 14 & 3 & & & 0 & \\
\hline Hylocomium splendens & 13 & 2 & & & & \\
\hline Cornus canadensis & 43 & 25 & 9 & & 0 & \\
\hline Vaccinium vitis-idaea & 6 & 0 & & & 0 & \\
\hline Betula papyrifera & 3 & 17 & & & 0 & 0 \\
\hline Linnaea borealis & 20 & 3 & 0 & & & \\
\hline Lycopodium aпnотіпит & 0 & 0 & & & 0 & \\
\hline Picea glauca & 29 & 21 & 3 & & 0 & 0 \\
\hline Anemone richardsonii & 2 & & 2 & & & \\
\hline Rubus arcticus & 4 & 0 & & & 0 & \\
\hline Rosa acicularis & 13 & 6 & 3 & & 0 & 0 \\
\hline Orthilia secunda & 0 & 0 & 0 & 0 & & \\
\hline Actaea rubra & 0 & 0 & 0 & 0 & & \\
\hline Trientalis europaea & 0 & 1 & 1 & & 0 & \\
\hline Rubus idaeus & 1 & 1 & 1 & & 0 & \\
\hline Viburnum edule & 2 & 14 & 16 & 2 & 1 & 0 \\
\hline Mertensia paniculata & 1 & 4 & 2 & 2 & 1 & 0 \\
\hline Galium triflorum & $\mathbf{0}$ & 1 & $\mathbf{0}$ & $\mathbf{0}$ & 1 & 0 \\
\hline Epilobium angustifolium & 1 & 1 & $\mathbf{0}$ & 4 & 1 & 0 \\
\hline Equisetum arvense & 5 & 6 & 6 & 11 & 4 & $\mathbf{0}$ \\
\hline Calamagrostis canadensis & 9 & 15 & 12 & 8 & 27 & 1 \\
\hline Populus balsamifera trichocarpa & 2 & 17 & 63 & & 1 & 2 \\
\hline Brachythecium sp. & 2 & 2 & 0 & 7 & 1 & 0 \\
\hline Alnus tenuifolia & 3 & 2 & 9 & & 49 & $\mathbf{0}$ \\
\hline Ribes triste & 0 & 1 & 2 & & 5 & \\
\hline Gymnосагрium dryopteris & & 2 & 2 & 2 & & \\
\hline Heracleum lanatum & & 4 & 12 & 4 & 0 & \\
\hline Moehringia lateriflora & & 0 & $\mathbf{0}$ & 0 & 0 & \\
\hline Alnus sinuata & & 3 & 3 & 5 & 7 & \\
\hline Pyrola asarifolia & 0 & 0 & 0 & $\mathbf{0}$ & & \\
\hline Oplopanax horridus & & 3 & 2 & & 1 & \\
\hline Athyrium filix-femina & & 0 & 17 & & 2 & \\
\hline Circaea alpina & & 0 & 0 & & 1 & \\
\hline Artemisia tilesii & & 2 & $\mathbf{0}$ & & 0 & $\mathbf{0}$ \\
\hline Salix alaxensis & & & & 30 & & 0 \\
\hline Sanguisorba stipulata & & & 0 & 7 & 1 & \\
\hline Streptopus amplexifolius & & 0 & 0 & 0 & 0 & \\
\hline Polemonium acutiflorum & & 0 & & & 1 & \\
\hline Aconitum delphinifolium & & 0 & & 3 & 0 & \\
\hline Ribes hudsonianum & & 1 & 0 & & 2 & 0 \\
\hline Thalictrum sparsiflorum & & 0 & 0 & & 1 & 0 \\
\hline Viola epipsila & & 0 & 1 & & 1 & \\
\hline Matteuccia struthiopteris & & & 1 & & 0 & \\
\hline Comarum palustre & & & & & 4 & \\
\hline Myrica gale & & & & & 2 & \\
\hline Agrostis scabra & 0 & & & & 0 & $\mathbf{0}$ \\
\hline Taraxacum sp. & & 0 & 0 & & 0 & $\mathbf{0}$ \\
\hline Ceratodon purpureus & & & & & & 1 \\
\hline Deschampsia caespitosa & & & & & & 1 \\
\hline Epilobium latifolium & & & & 1 & & 1 \\
\hline Achillea millefolium & & & & & & $\mathbf{0}$ \\
\hline Astragalus alpinus & 0 & & & & & 0 \\
\hline Taraxacum officinale & & & 0 & 0 & & 0 \\
\hline sample size & 5 & 8 & 5 & 3 & 7 & 5 \\
\hline
\end{tabular}


Table 15. Mean cover (\%) of the most abundant species within coastal ecotypes on Fort Richardson, south-central Alaska, 2001. Bold numbers represent frequencies $\geq 60 \%$ within ecotype, blank when absent, and $0=<0.5 \%$ cover. Data from Racine et al. (1993).

\begin{tabular}{|c|c|c|c|c|c|c|c|c|c|c|c|}
\hline Taxon & $\begin{array}{l}\Omega \\
0 \\
0 \\
0 \\
0 \\
0 \\
0 \\
0 \\
0\end{array}$ & 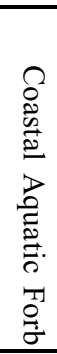 & 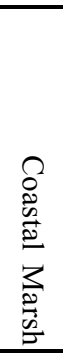 & 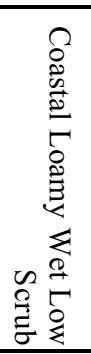 & 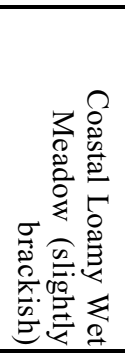 & 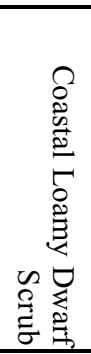 & 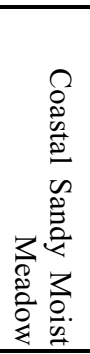 & 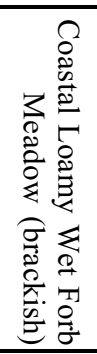 & 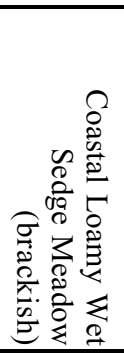 & 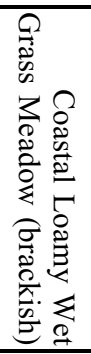 & 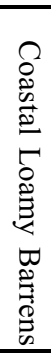 \\
\hline Potamogeton pectinatus & 0 & 21 & & & & & & 0 & & & \\
\hline Zannichellia palustris & 2 & 26 & 5 & & & & & 0 & & & \\
\hline Scirpus validus & 1 & 1 & 6 & & 0 & & & & & & \\
\hline Scirpus paludosus & 0 & 0 & 29 & & 1 & & & & & & 0 \\
\hline Ruppia spiralis & 3 & & 1 & & 0 & & & & & & \\
\hline Myriophyllum exalbescens & & 7 & & & 1 & & & & & & \\
\hline Hippuris tetraphylla & & 9 & 6 & & 0 & & & 0 & 1 & & 1 \\
\hline Carex mackenziei & & 1 & & & 5 & & & & & & \\
\hline Carex pluriflora & & 1 & & & 1 & 3 & & & & & \\
\hline Eleocharis kamtschatica & & & 0 & & 0 & & & & & & \\
\hline Cicuta virosa & & & & 0 & 0 & & & & & & \\
\hline Carex aquatilis & & & & & 1 & & & 0 & & 0 & 0 \\
\hline Galium trifidum & & & & & 0 & & & & & & \\
\hline Hierchloe odorata & & & & & 0 & & & & & & \\
\hline Lathyrus palustris & & & & & 0 & & & 1 & & & \\
\hline Hordeum brachyantherum & & & & & 0 & & & 0 & & & \\
\hline Comarum palustre & & & & 6 & 0 & & & 0 & & & \\
\hline Myrica gale & & & & 44 & 0 & & & & & & 0 \\
\hline Calamagrostis sp. & & & & 19 & 0 & 1 & 0 & 0 & 0 & & 0 \\
\hline Arctagrostis sp. & & & & & & & & 0 & & & \\
\hline Angelica lucida & & & & & & & 2 & & & & \\
\hline Salix ovalifolia & & & & 3 & 0 & 28 & 1 & & & & \\
\hline Glaux maritima & & & & & 0 & & 2 & 0 & 0 & & \\
\hline Poa eminens & & & & & 0 & & & 1 & & & \\
\hline Leymus mollis & & & & & & 2 & 32 & 0 & & & \\
\hline Triglochin palustris & & 0 & 0 & & 0 & & 0 & 0 & 0 & 0 & 0 \\
\hline Carex lyngbyaei & & 0 & 0 & 8 & 47 & 15 & 1 & 1 & 1 & & 1 \\
\hline Potentilla egedii & & & & 2 & 6 & 10 & 15 & 9 & 4 & 1 & 1 \\
\hline Plantago maritima & & & & & 0 & 3 & 11 & 18 & 0 & 1 & 0 \\
\hline Triglochin maritimum & & & & & 2 & 3 & 6 & 19 & 10 & 8 & 2 \\
\hline Carex ramenskii & & & & & 1 & & 0 & 1 & 43 & 0 & 0 \\
\hline Puccinellia nutkaensis & & & & & 0 & & 0 & 2 & 0 & 29 & 2 \\
\hline Atriplex gmelini & & & & & 0 & & 0 & 0 & 0 & 2 & 0 \\
\hline Stellaria humifusa & & & & & 0 & & & 0 & 0 & & 0 \\
\hline Salicornia europaea & & & & & & & & 0 & & 1 & 0 \\
\hline Puccinellia phryganodes & & & & & & & & 0 & 0 & 3 & \\
\hline Ranunculus cymbalaria & & & & & & & 0 & 0 & & & 0 \\
\hline Rumex arcticus & & & & & & & & & & & 0 \\
\hline Dodecatheon pulchellum & & & & & & & & & & & 0 \\
\hline sample size & 9 & 10 & 10 & 5 & 83 & 3 & 17 & 129 & 36 & 24 & 56 \\
\hline
\end{tabular}




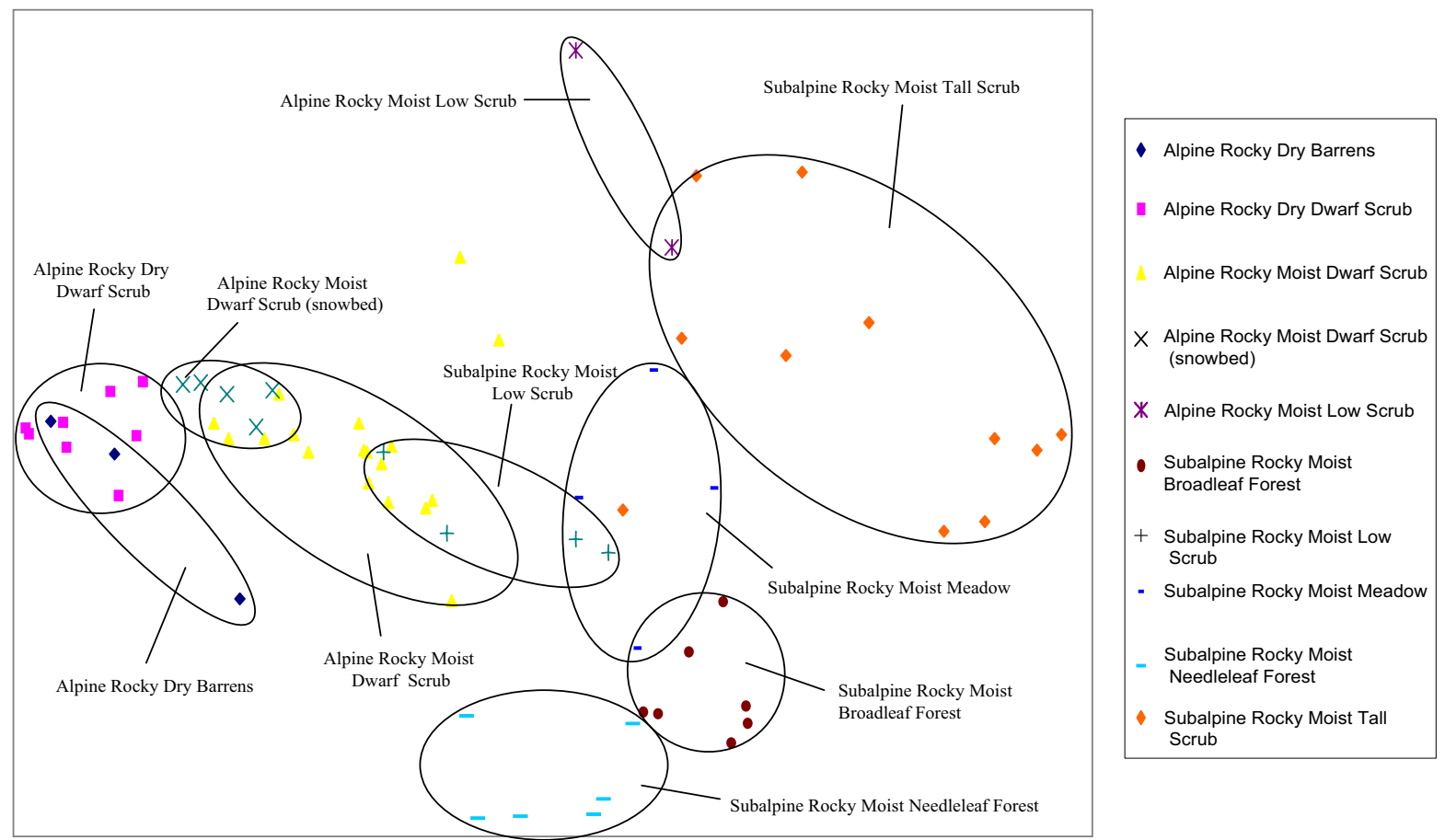

Figure 20. Detrended correspondence analysis of alpine and subalpine plots and associated plant species analyzed from Fort Richardson, south-central Alaska, 2001. Ellipses represent central tendencies of ecotypes in species space.
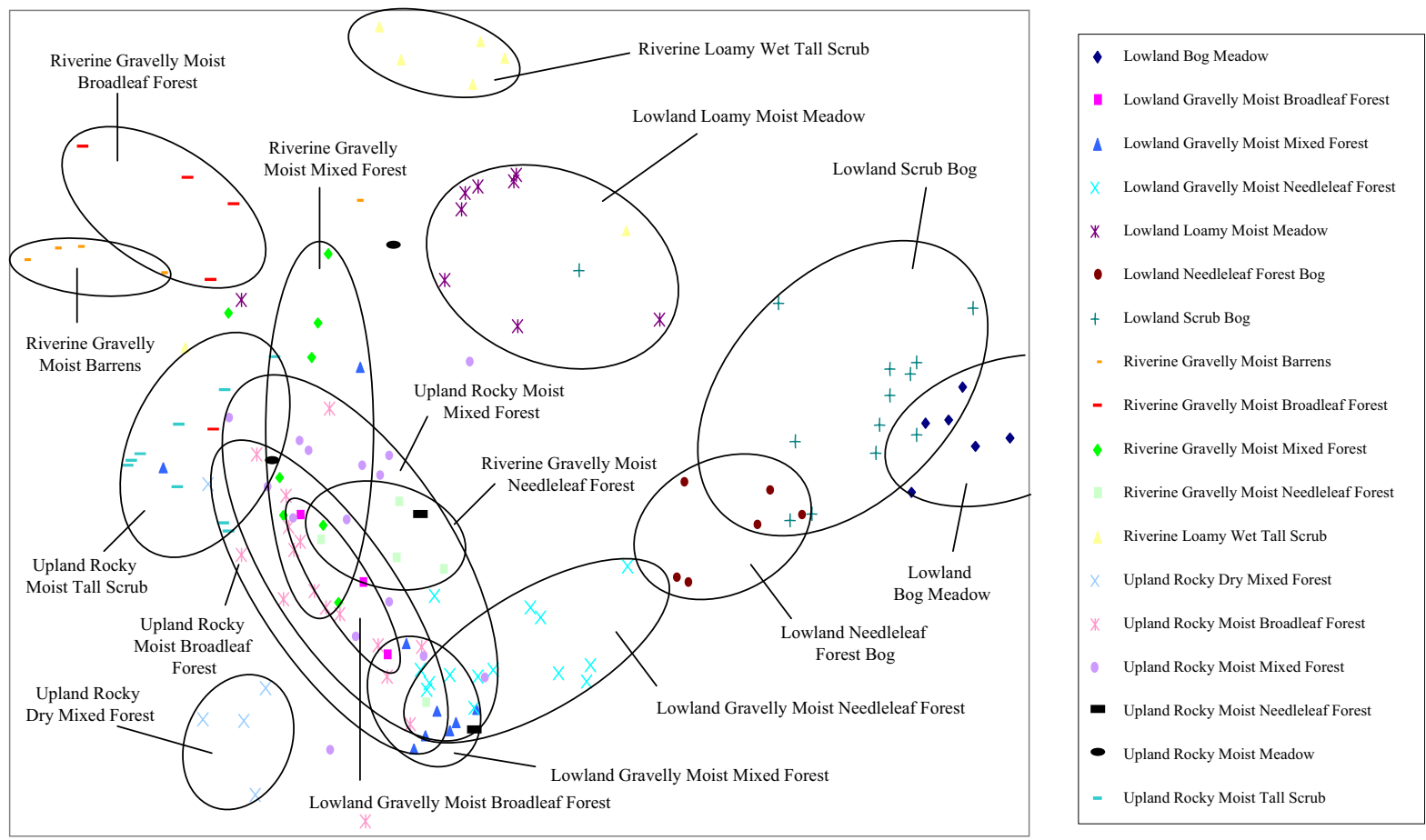

Figure 21. Detrended correspondence analysis of lowland, riverine, and upland plots and associated plant species analyzed from Fort Richardson, south-central Alaska, 2001. Ellipses represent central tendencies of ecotypes in species space. 
physiography and soil texture, and because they have different origins.

Lowland ecotypes on organic soils (bogs) supported a variety of growth forms, but were remarkable for their high abundance of mosses (Fig. 19). Floristically, the various bog types had many species in common (Table 13, Fig. 21), but were very different from the upland rocky and lowland gravelly ecotypes (Table 12). Common bog species included Ledum palustre decumbens, Chamaedaphne calyculata, Empetrum nigrum, Betula nana, Oxycoccus microcarpus, Andromeda polifolia, Rubus chamaemorus, and Sphagnum spp. Lowland Needleleaf Forest Bog was the only forest ecotype on thick peat. It was distinguished by the presence of Picea mariana and differentiated from Lowland Moist Needleleaf Forest by the presence of Sphagnum spp. Lowland Scrub Bog had few trees ( $<25 \%$ cover), which typically were stunted. Two plant associations were identified within the Lowland Scrub Bog ecotype, Myrica gale-Calamagrostis canadensis and Betula nana-Rubus chamaemorus (Table 8). The Myrica gale association usually had slightly higher $\mathrm{EC}$ and $\mathrm{pH}$ than the Betula nana association. Lowland Bog Meadows were dominated by sedges and Sphagnum mosses. While this ecotype was associated with pond margins and had characteristics of a poor fen (dominated by both sedges and Sphagnum), we used the term bog to remain consistent with the AVC name, Subarctic Lowland Sedge-Moss Bog Meadow.

Lacustrine ecotypes included ponds with open water, and shallow shorelines with submergent or floating vegetation. Lacustrine Aquatic Forb was differentiated from Lakes and Ponds by the presence of Nuphar polysepalum and Potamogeton spp. Other aquatic species that were characteristic of lacustrine ecotypes included Menyanthes trifoliata, Equisetum fluviatile, and Comarum palustre.

Riverine ecotypes occurred primarily on welldrained riverine gravels and sands, and ranged from forb-dominated barrens at the earliest successional stage to needleleaf forests at the latest successional stage (Fig. 19). Large differences in floristic composition accompanied the successional trend from barrens to needleleaf forest (Table 14). Species that were common to nearly all riverine ecotypes included Populus balsamifera trichocarpa, Viburnum edule, Alnus tenuifolia, Mertensia paniculata, Galium triflorum, Epilobium angustifolium, Equisetum arvense, Calamagrostis canadensis, and Brachythecium spp. Riverine Gravelly Moist Barrens were barren to partially vegetated active riverbars that supported scattered, early successional species, including Epilobium latifolium, Populus balsamifera trichocarpa seedlings, Artemisia tilesii, Agrostis scabra, and Deschampsia caespitosa. River- ine Gravelly Moist Tall Scrub occurred on steeper floodplains in the mountains, was dominated by Salix alaxensis, and was differentiated from Riverine Loamy Wet Tall Scrub by the presence of Sanguisorba stipulata and the absence of Alnus tenuifolia. Riverine Loamy Wet Tall Scrub was characterized by wet loamy soils on inactive channel deposits, was dominated by Alnus tenuifolia and was differentiated from Upland Rocky Moist Tall Scrub by the presence of Thalictrum sparsiflorum. Riverine Gravelly Moist Broadleaf Forest was dominated by Populus balsamifera trichocarpa and differentiated from Riverine Gravelly Moist Mixed Forest by the prevalence of Heracleum lanatum. Riverine Gravelly Mixed Forest was dominated by both Populus balsamifera trichocarpa and Picea glauca and was differentiated from Riverine Moist Broadleaf Forests by frequent occurrence of Cornus canadensis. Riverine Gravelly Moist Needleleaf Forests, the oldest successional stage on inactive floodplains, was dominated by Picea glauca and differentiated from all other riverine ecotypes by the prevalence of Vaccinium vitis-idaea. In these later stages of floodplain succession, the species composition was similar to that of upland and lowland forests (Fig. 21).

Coastal ecotypes were dominated mostly by graminoids and forbs tolerant of brackish conditions (Fig. 19). Strong gradients in both salinity and water levels resulted in distinct zonation in floristics across the coastal areas, with brackish ecotypes on active tidal flats and slightly brackish ecotypes on inactive tidal flats (Table 15, Fig. 22). All the brackish ecotypes were floristically similar; common species included Potentilla egedii, Triglochin palustris, Carex lyngbyaei, Plantago maritima, Triglochin maritimum, Puccinellia nutkaënsis, Carex ramenskii, and Atriplex gmelini. The Coastal Loamy Barrens ecotype was sparsely vegetated. Coastal Loamy Brackish Wet Grass Meadow was dominated by Puccinellia nutkaënsis, Coastal Loamy Brackish Wet Forb Meadow was dominated by Plantago maritima, Coastal Loamy Brackish Wet Sedge Meadow was dominated by Carex ramenskii, and Coastal Sandy Moist Meadow was dominated by Leymus mollis. Coastal Loamy Moist Dwarf Scrub was distinguished by the dominance of Salix ovalifolia, which was rare in other ecotypes. Among slightly brackish ecotypes, Coastal Loamy Slightly Brackish Wet Meadow was dominated by Carex lyngbyaei and had a wide range of associated species. A second plant association (Carex mackenziei-Eleocharis kamtschatica) was identified for this ecotype, but it was restricted to slightly brackish depressions or pond margins with organic-rich soils. Coastal Loamy Wet Low Scrub, which occurs in the transition zone to non-brackish lowland ecotypes, had a relatively distinct plant association dominated by 


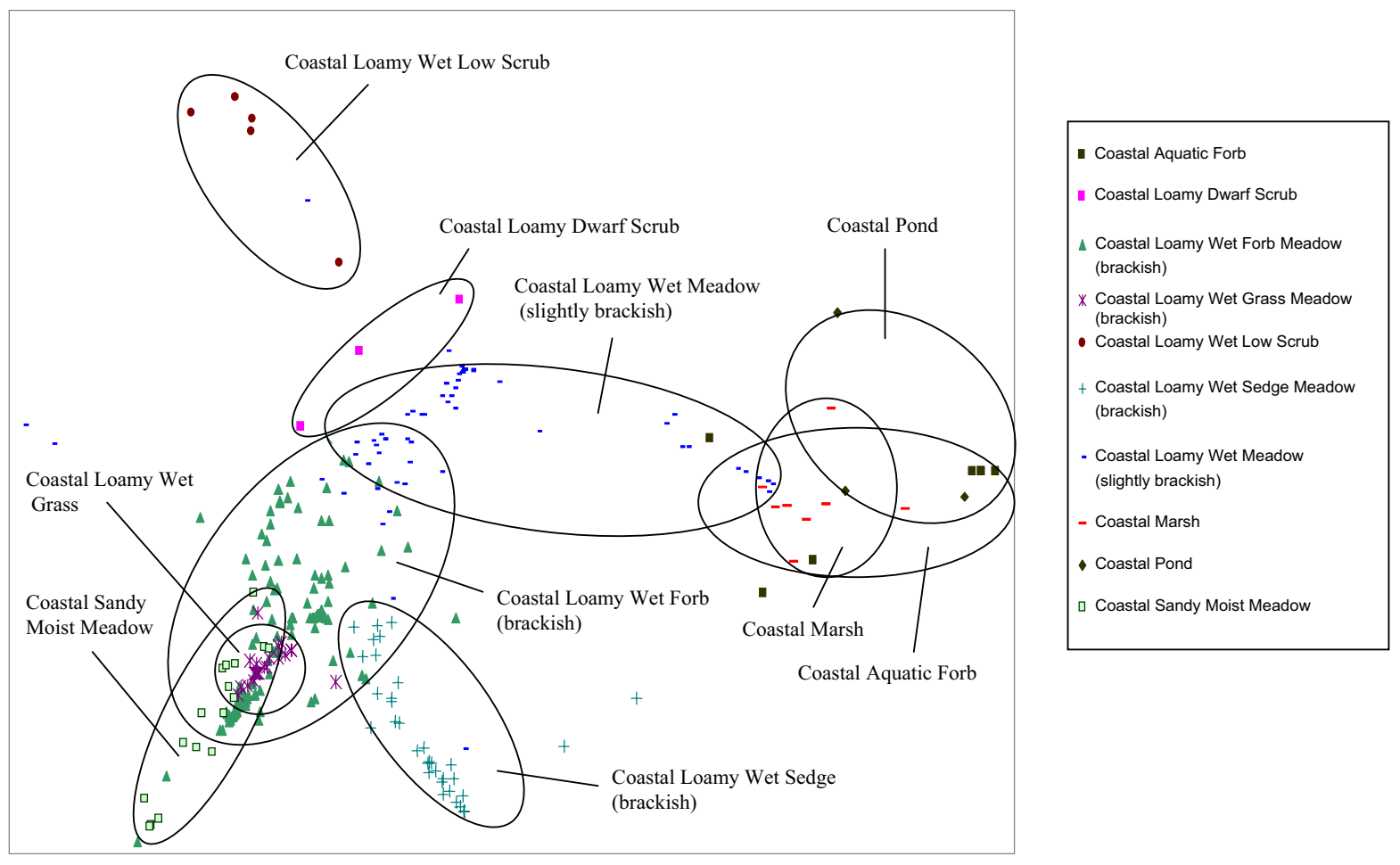

Figure 22. Detrended correspondence analysis of coastal plots and associated plant species analyzed from Fort Richardson, south-central Alaska, (data from Racine et al. 1993). Ellipses represent central tendencies of ecotypes in species space.

Myrica gale (Fig. 19). This type was similar to Lowland Scrub Bog, but was differentiated from it by the presence of Carex lyngbyaei and the absence of Sphagnum spp. These ecotypes, and their associated plants, were developed from data collected by Racine et al. (1993) and are consistent with the plant zonation found on the Susitna Flats by Vince and Snow (1984).

The three aquatic coastal types were floristically similar; all were dominated by forbs and sedges (Fig. 19), and varied only in relative abundances of the dominant species (Table 15, Fig. 22). Coastal Ponds were too sparsely vegetated to be assigned a plant association (Table 8). Coastal Aquatic Forb, on the other hand, included two closely related plant associations: one dominated by Zannichellia palustris and Potamogeton pectinatus and one dominated by Hippuris tetraphylla. The second association typically occurred at the transition between pond and marsh, or in isolated shallows. Finally, Coastal Marsh was dominated by Scirpus paludosus with no clear differential species.

The floristic composition of human-modified ecotypes was not analyzed, because these types occurred over a range of physiographic conditions and disturbance regimes. Instead, they were classified on the basis of vegetation structure, to help differentiate successional stages in response to disturbance (Tables 8 and
16). Generally, disturbances with soil removal created conditions suitable for early successional species. Barren and partially vegetated areas supported scattered individuals of Agropyron sp., Plantago major, Trifolium spp., Potentilla spp., and Ceratodon purpureus. Many of the same species occurred in Human Modified Meadows, in addition to Hordeum jubatum, Achillea millifolium, Taraxacum spp., Epilobium angustifolium, and Calamagrostis canadensis. Human Modified Scrub occurred at older or less severely disturbed sites and was characterized by the presence of low and tall shrubs, and tree saplings. Willows, particularly Salix scouleriana and S. bebbiana, were commonly observed resprouting in lowland areas that had been cleared to enhance moose habitat, whereas Alnus sinuata was more common in older clearings on uplands. Human Modified Forest was similar to young, undisturbed forest communities and was dominated by Picea glauca, Populus balsamifera trichocarpa, and Betula papyrifera.

Environmental characteristics To evaluate the influence of environmental factors on the distribution of ecotypes, we compared six key environmental parameters (elevation, cumulative organic depth, depth to gravel, $\mathrm{pH}, \mathrm{EC}$, and water depth) among ecotypes 
Table 16. Classification, description, and areal extent of disturbances mapped on Fort Richardson, south-central Alaska, 2001.

\begin{tabular}{|c|c|c|c|c|}
\hline \multirow[b]{2}{*}{ Disturbance } & \multirow[b]{2}{*}{ Description } & \multicolumn{3}{|c|}{ Area } \\
\hline & & acre & ha & $\%$ \\
\hline $\begin{array}{l}\text { Clearing for habitat } \\
\text { enhancement (Hch) }\end{array}$ & $\begin{array}{l}\text { Areas intentionally altered to encourage growth of species } \\
\text { (e.g. young willow) suitable for moose browse. }\end{array}$ & 1349 & 546 & 2.2 \\
\hline $\begin{array}{l}\text { Clearing with no soil } \\
\text { removal (Hen) }\end{array}$ & $\begin{array}{l}\text { Areas where vegetation was cut in such a way that there was } \\
\text { little or no disturbance of the soil surface. Woody } \\
\text { vegetation can re-sprout from rootstock. }\end{array}$ & 2378 & 962 & 3.8 \\
\hline $\begin{array}{l}\text { Clearing with soil removal } \\
\text { (Hcs) }\end{array}$ & $\begin{array}{l}\text { Areas that were cleared in such a way that a substantial } \\
\text { portion }(<50 \mathrm{~cm}) \text { of surface soil was removed or disturbed. } \\
\text { Vegetation re-establishes primarily from seed. }\end{array}$ & 1656 & 670 & 2.7 \\
\hline Undifferentiated Trail (Ht) & $\begin{array}{l}\text { Cleared paths for mechanized or foot travel, usually without } \\
\text { added fill. }\end{array}$ & 30 & 12 & $<0.1$ \\
\hline Gravel Fill (Hfg) & Gravel added above soil grade. & 69 & 28 & 0.1 \\
\hline Gravel Road (Hfgr) & $\begin{array}{l}\text { Established, maintained roads, constructed by addition of } \\
\text { minimal to substantial amounts of gravel fill. }\end{array}$ & 546 & 221 & 1.0 \\
\hline Gravel Airstrip (Hfga) & $\begin{array}{l}\text { Long, narrow clearings amended with gravel fill, and } \\
\text { commonly with cleared or partially brushed perimeters. }\end{array}$ & 14 & 6 & $<0.1$ \\
\hline Gravel Pad (Hfgp) & $\begin{array}{l}\text { Clearings, usually associated with gravel roads, and with } \\
\text { similar surface characteristics. }\end{array}$ & 40 & 16 & 0.1 \\
\hline Paved Road (Hfgrp) & $\begin{array}{l}\text { Main arterial roads constructed with substantial fill above } \\
\text { soil grade and surfaced with pavement or chip-seal. }\end{array}$ & 243 & 98 & 0.4 \\
\hline Paved Airstrip (Hfgap) & $\begin{array}{l}\text { Long, narrow clearings amended with gravel fill and } \\
\text { surfaced with pavement. Approaches and perimeters are } \\
\text { brushed and cleared. }\end{array}$ & 17 & 7 & $<0.1$ \\
\hline Undifferentiated Fill (Hf) & $\begin{array}{l}\text { Fill of undetermined texture (can include sod, gravel, or } \\
\text { medium grained soil). }\end{array}$ & 34 & 14 & 0.1 \\
\hline Ditch (Hwd) & $\begin{array}{l}\text { Long, narrow excavations, usually designed to channel } \\
\text { excess surface run-off. }\end{array}$ & 2 & 1 & $<0.1$ \\
\hline $\begin{array}{l}\text { Water-filled Excavation } \\
\text { (Hwe) }\end{array}$ & $\begin{array}{l}\text { An artificially constructed depression or pit that holds } \\
\text { water. }\end{array}$ & 8 & 3 & $<0.1$ \\
\hline Berms, Spoil Piles (Hfb) & $\begin{array}{l}\text { Fill in relatively small mounds, often related to mining or } \\
\text { excavation. }\end{array}$ & 2 & 1 & $<0.1$ \\
\hline $\begin{array}{l}\text { Excavation/Pits } \\
\text { (undifferentiated) (He) }\end{array}$ & $\begin{array}{l}\text { Areas in which }>50 \mathrm{~cm} \text { of surface soil has been removed. } \\
\text { Usually, little or no fine material remains and underlying } \\
\text { rock or gravel is exposed. Excavations in this class are of } \\
\text { undetermined purpose. }\end{array}$ & 309 & 125 & 0.5 \\
\hline Disturbance Complex (DC) & $\begin{array}{l}\text { An area affected by three or more disturbance types, none of } \\
\text { which is dominant, and all are too small to differentiate } \\
\text { individually. }\end{array}$ & 1274 & 515 & 2.1 \\
\hline Undisturbed & Land on which no human modification is evident. & 54,025 & 21,863 & 87.1 \\
\hline Total & & 61,996 & 25,089 & 100 \\
\hline
\end{tabular}

(Fig. 23 and 24). In the following discussion we highlight some of the important differences in environmental parameters among ecotypes. Relationships of environmental parameters to distributions of individual plant species are illustrated in Figures 25 and 26, but are not discussed in detail. For each species, the mean value of each parameter was based on plots where the species occurred with $>0.5 \%$ cover. No environmental data were available for coastal ecotypes.

Elevations of the survey plots ranged from 4 to 1328 $\mathrm{m}$ above sea level (Fig. 23). Alpine ecotypes usually were above $800 \mathrm{~m}$, with Alpine Rocky Dry Barrens and Alpine Rocky Dry Dwarf Scrub typically above 1100 $\mathrm{m}$. Subalpine ecotypes occurred mainly between 600 and $800 \mathrm{~m}$. A few species were limited to the subalpine zone, particularly Tsuga mertensiana, Valeriana sitchensis, Geranium erianthum, and Aconitum delfinifolium (Fig. 25). Mean elevations of upland, lowland, and riverine ecotypes were below $300 \mathrm{~m}$, with most plots below $100 \mathrm{~m}$. Of this group, Upland Rocky 


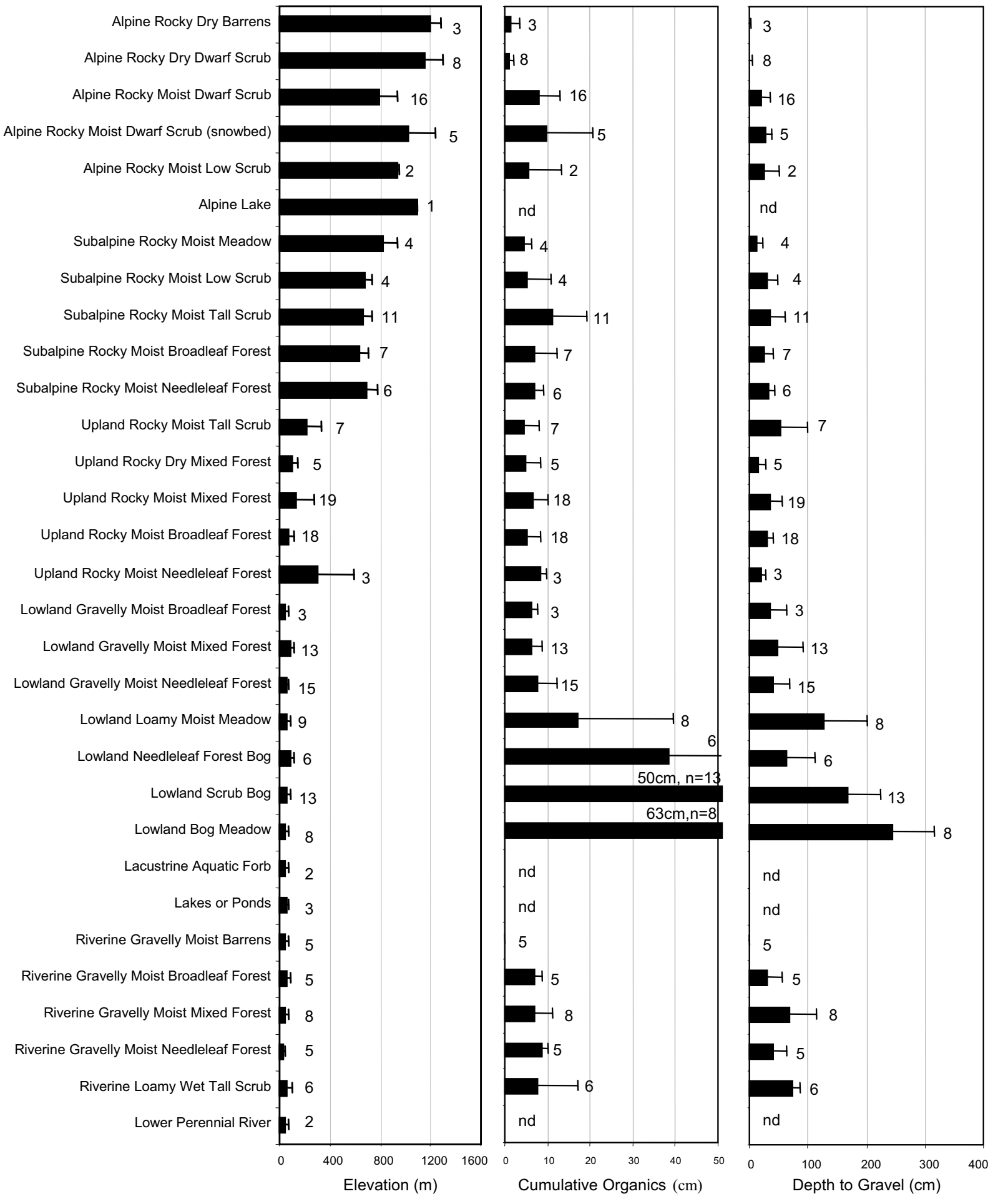

Figure 23. Mean ( \pm SD) elevation, organic matter thickness, and depth to gravel of ecotypes on Fort Richardson, south-central Alaska, 2001. Sample sizes listed to right of bars. 


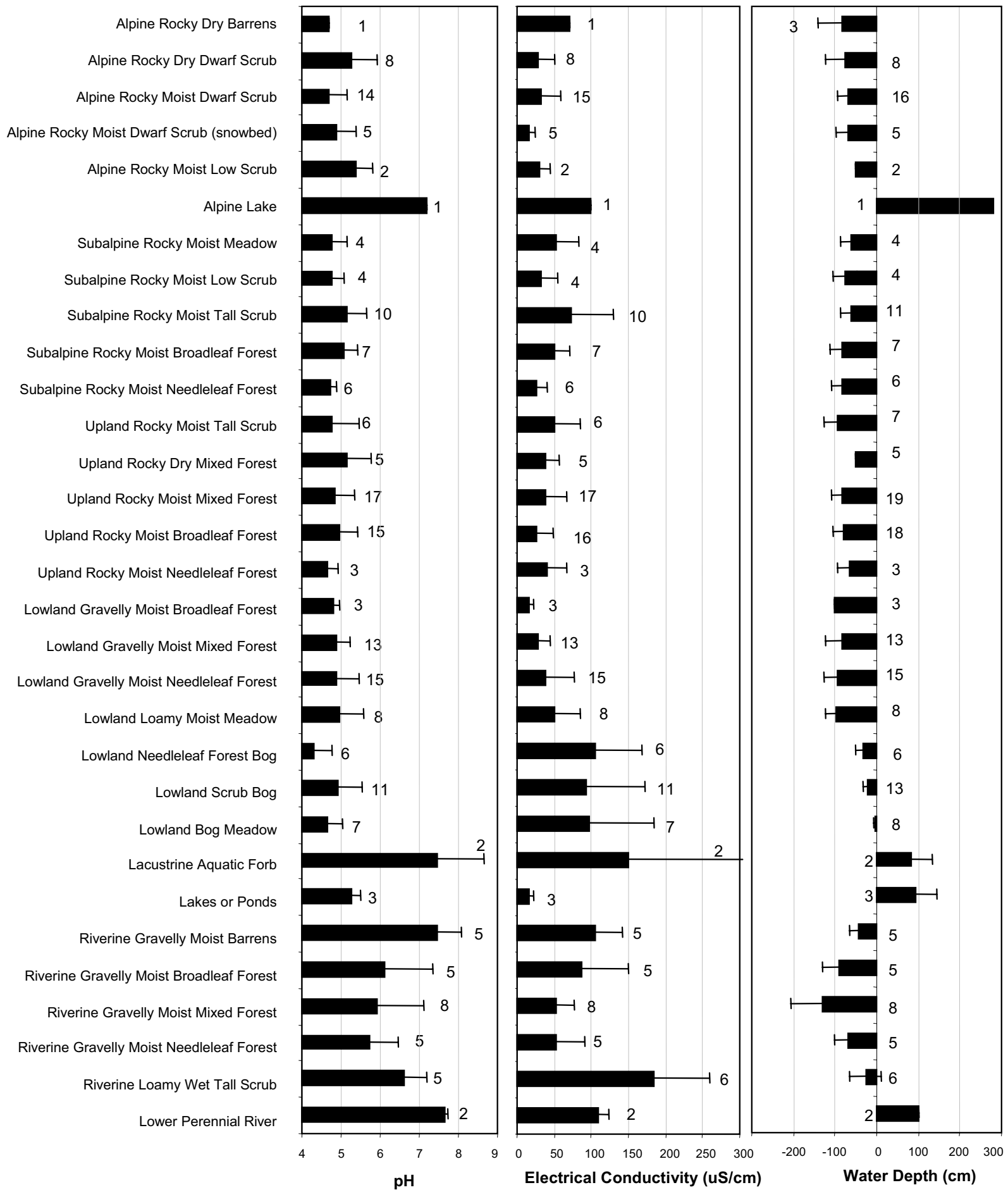

Figure 24. Mean ( $\pm \mathrm{SD}) \mathrm{pH}$, electrical conductivity (EC), and water depth (negative when below ground) for ecotypes on Fort Richardson, south-central Alaska, 2001. Sample sizes listed to right of bars. 

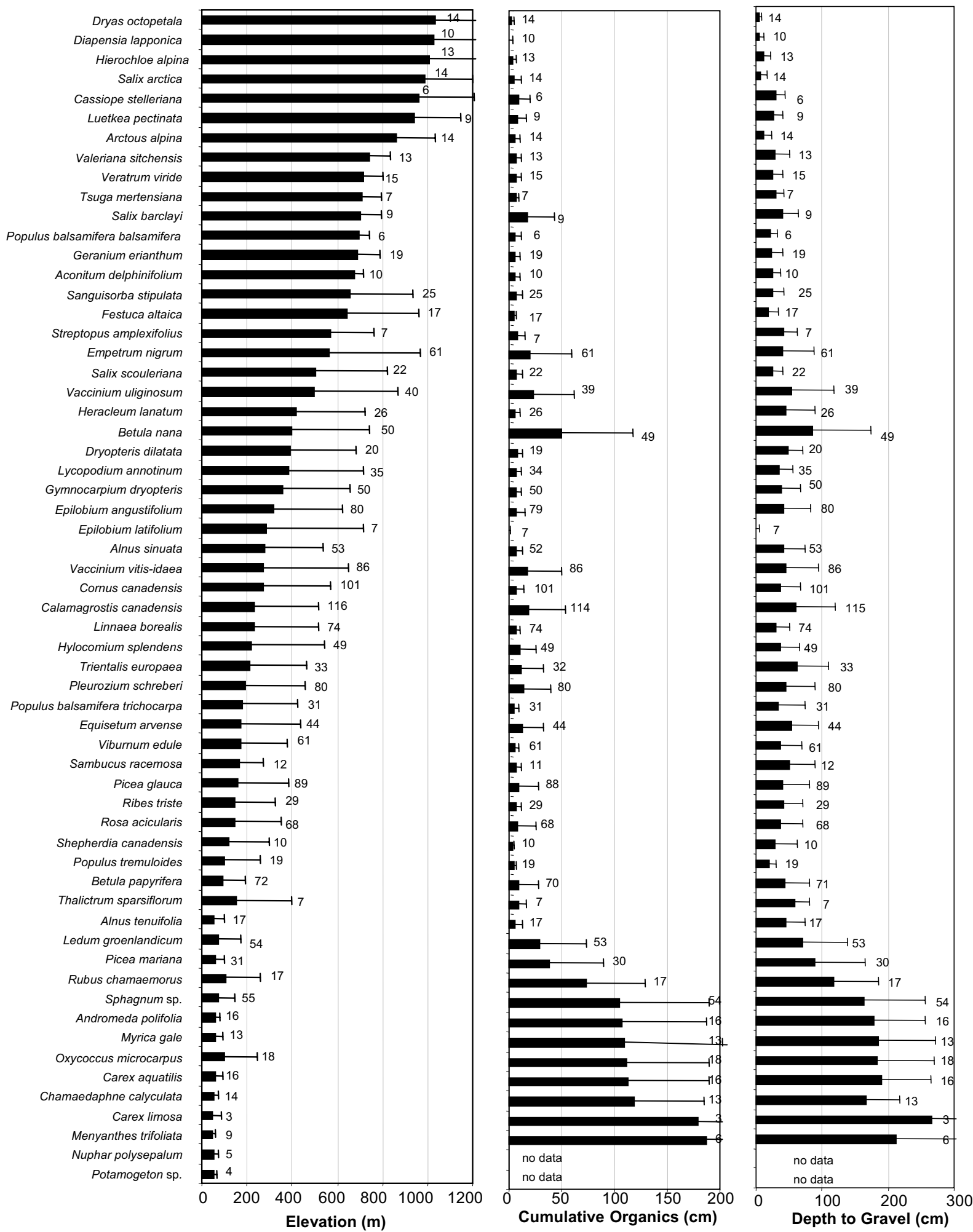

Figure 25. Mean ( $\pm S D$ ) elevation, organic matter thickness, and depth to gravel for abundant plant species on Fort Richardson, south-central Alaska, 2001. Sample sizes listed to right of bars. 
Dryas octopetala

Diapensia lapponica

Hierochloe alpina

Salix arctica

Cassiope stellerian

Luetkea pectinata

Arctous alpina

Valeriana sitchensis

Veratrum viride

Tsuga mertensiana

Salix barclay

Populus balsamifera

Geranium erianthum

Aconitum delphinifolium

Sanguisorba stipulata

Festuca altaica

Streptopus amplexifolius

Empetrum nigrum

Salix scouleriana

Vaccinium uliginosum

Heracleum lanatum

Betula nana

Dryopteris dilatata

Lycopodium annotinum

Gymnocarpium dryopteris

Epilobium angustifolium

Epilobium latifolium

Alnus sinuata

Vaccinium vitis-idae

Cornus canadensis

Calamagrostis canadensis

Linnaea borealis

Hylocomium splenden

Trientalis europaea

Pleurozium schreber

Populus balsamifera trichocarpa

Equisetum arvense

Viburnum edule

Sambucus racemosa

Picea glauca

Ribes triste

Rosa acicularis

Shepherdia canadensis

Populus tremuloides

Betula papyrifera

Thalictrum sparsiflorum

Alnus tenuifolia

Ledum groenlandicum

Picea mariana

Rubus chamaemorus

Sphagnum sp.

Andromeda polifolia

Myrica gale

Oxycoccus microcarpus

Carex aquatilis

Chamaedaphne calyculata

Carex limosa

Menyanthes trifoliata

Nuphar polysepalum

Potamogeton $\mathrm{sp}$

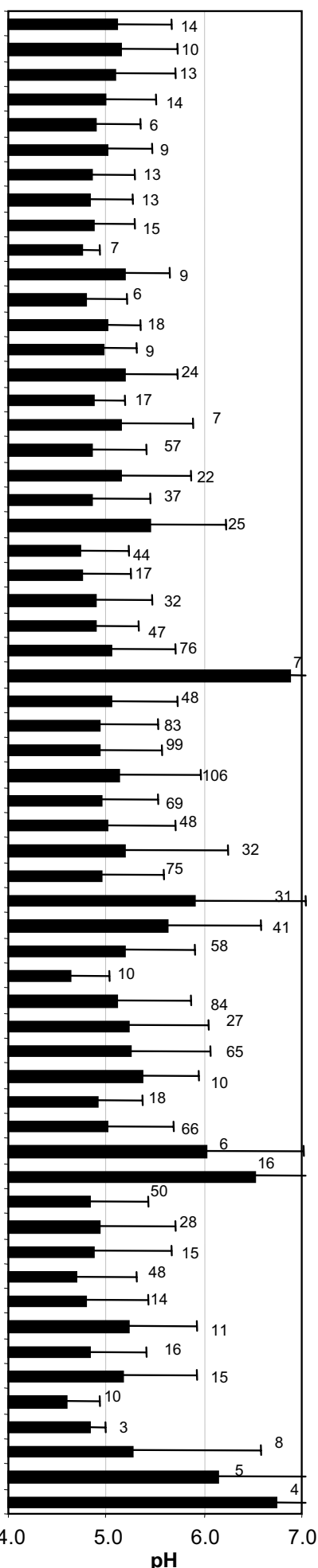

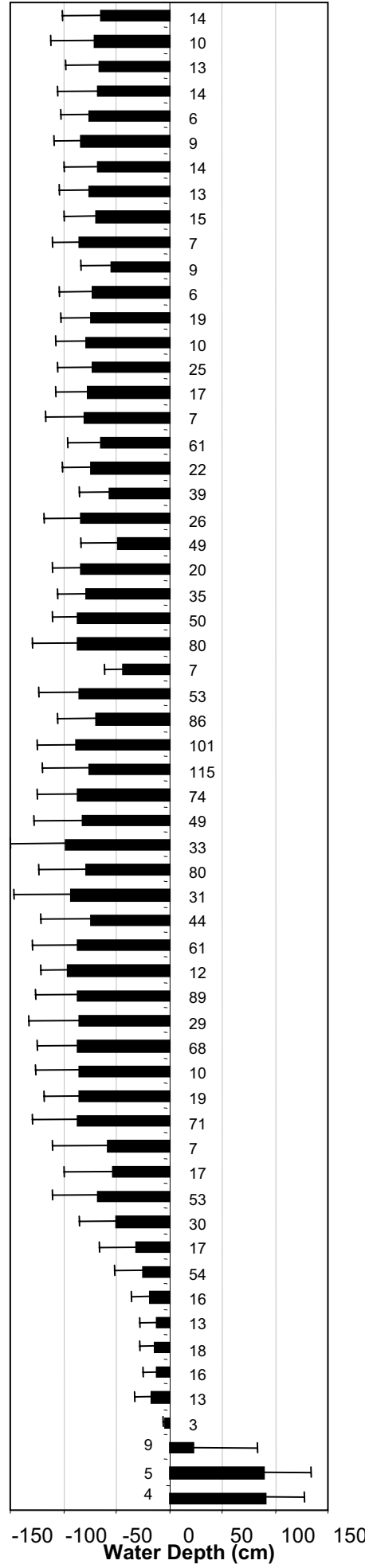

Figure 26. Mean ( \pm SD) pH, electrical conductivity (EC), and water depth (negative when below ground) for abundant plant species on Fort Richardson, south-central Alaska, 2001. Sample sizes listed to right of bars. 
Moist Needleleaf Forest had the highest average elevation $(296 \mathrm{~m})$.

Organic matter depth is a general indicator of rates of decomposition and nutrient cycling, and surface stability. Undisturbed soils accumulate organic material over time, particularly in depressions and areas where poor drainage may slow plant decomposition. Surface organic matter depth (uninterrupted organic horizons at the surface) and cumulative organic depth (sum of organic horizons found within $40 \mathrm{~cm}$ of the soil surface) were both measured. Cumulative organic depths generally averaged $<10 \mathrm{~cm}$ except in the bogs (Fig. 23). By definition, the surface organic layer of a bog should be $\geq 40 \mathrm{~cm}$ thick (NWWG 1988). The mean depth of the surface organic layer was $38 \mathrm{~cm}$ in Lowland Needleleaf Forest Bogs, $50 \mathrm{~cm}$ in Lowland Scrub Bogs, and $63 \mathrm{~cm}$ in Lowland Bog Meadows. Cumulative organic depth in Lowland Loamy Moist Meadow was highly variable, indicating variable histories of drainage conditions and sedimentation in the lacustrine basins. Late-successional forest ecotypes on rocky upland, gravelly lowland, and gravelly riverine areas had slightly thicker organic accumulations (averaging 8-9 $\mathrm{cm}$ ) than did early successional ecotypes (averaging 5$7 \mathrm{~cm}$ ). Alpine Rocky Dry Barrens, and Alpine Rocky Dry Dwarf Scrub had the thinnest accumulations of organic matter (average $<2 \mathrm{~cm}$ ).

Depth to gravel (rock) is important for evaluating drainage and soil moisture, and for assessing accumulation of eolian and fluvial fine-grained material. Gravel was $<3 \mathrm{~cm}$ from the surface for Alpine Rocky Dry Barrens, Alpine Rocky Dry Dwarf Scrub, and Riverine Barrens (Fig. 23). Depth to gravel was greatest in organic and loamy lowlands, averaging $>100 \mathrm{~cm}$ in Lowland Loamy Moist Meadow, Lowland Scrub Bog, and Lowland Bog Meadow. Most other ecotypes were on colluvium, glacial till, or channel deposits, with average depth to gravel typically ranging from 30 to $50 \mathrm{~cm}$. An eolian silt cap was widespread across the glaciated lowlands, but it was too thin and inconsistent to map as a separate geomorphic unit or to be useful for differentiating ecotypes.

Most environments on Fort Richardson were strongly acidic $(\mathrm{pH}<5.5$ for open water, free soil water, or a soil paste from 10-cm depth) (Fig. 24). The lowest $\mathrm{pH}$ values were measured in Lowland Needleleaf Forest Bog (4.3), Alpine Rocky Dry Barrens (4.7), and Alpine Rocky Moist Dwarf Scrub (4.7). In contrast, riverine ecotypes were circumneutral (5.5-7.4), although there was a distinct trend of decreasing soil $\mathrm{pH}$ in later successional stages. Waterbodies and aquatic ecotypes generally had $\mathrm{pH}$ values between 7 and 8 . Species occurring in circumneutral environments included
Epilobium latifolium, Thalictrum sparsiflorum, Alnus tenuifolia, Nuphar polysepalum, and Potamogeton spp. (Fig. 26). Species that occurred mainly on acidic sites included Sambucus racemosa, Chamaedaphne calyculata, Betula nana, and Sphagnum spp.

Electrical conductivity (EC) provides a good measure of soil or water salinity. EC was relatively low for most ecotypes (mean $<\sim 100 \mu \mathrm{S} / \mathrm{cm}$ ), indicating highly leached soil conditions (Fig. 24). Although we did not conduct any field surveys in coastal areas, coastal ecotypes on Active Tidal Flats probably had the highest EC levels based on relationships in other coastal areas (Jorgenson 2000). In non-coastal sites, EC values tended to be slightly elevated in lowland bogs and lacustrine margins, where groundwater movement probably affects dissolved solute concentrations, and in early successional riverine ecotypes, where sedimentation probably contributes to higher soil cation concentrations.

Water depths (positive when above ground, negative when below ground) were measured where possible, but often were estimated for aquatic sites (river, lakes, ponds, aquatic forb) or assigned an arbitrary large value $(-100$ or $-200 \mathrm{~cm})$ for well-drained rocky soils (Fig. 24). Among the terrestrial classes, water was closest to the surface $(<-40 \mathrm{~cm})$ for Lowland Bog Meadow, Lowland Scrub Bog, Riverine Loamy Wet Tall Scrub, and Lowland Needleleaf Forest. For nearly all other ecotypes, average water depths were $\sim-100 \mathrm{~cm}$, an arbitrary number that does not reflect true depth to water, but does indicate that water was not found near the surface.

Ecosystem dynamics Ecosystems continually change over time, in response to both changes in the physical environment and internal forces that alter microclimatic or soil conditions (Pickett et al. 1989). Disturbances caused by physical forces such as fire or flooding can alter the entire community, or may affect only a few species that are sensitive to a particular agent of change. Disturbances usually recur over time and across the landscape, typically creating early-successional ecosystems that provide evidence of the extent and frequency of disturbances. Thus, the occurrence of large single-aged stands of trees indicates rare, but intense, large-scale disturbances, while the presence of a variety of age classes suggests more frequent, smallerscale disturbances. Knowledge of the effects of disturbance regimes on ecosystem patterns and processes can have important implications for ecosystem management. Managing human activities to be compatible with natural disturbance regimes can help maintain the ability of ecosystems to recover over time.

By examining the nature and distribution of ecotypes 
in the study area, we identified the following disturbance types affecting the dynamics of ecosystems: fire, insect outbreaks, fluvial processes associated with channel migration and flooding, landslides and rockfalls on steep slopes in the mountains, coastal flooding, and human modifications. Thermokarst is a major factor in interior Alaska, but is of little importance in the Anchorage area. Other disturbance types, such as volcanoes, earthquakes, storms and windfalls, and drought, also affect the landscape, but are sufficiently rare or localized that their effects are not readily apparent in our ecological mapping. In the following discussion we identify the ecotypes associated with the various disturbance types, and discuss the general conceptual models that have been developed to describe the responses of ecosystems to disturbance.

Fire. Fire is a frequent and widespread disturbance type in interior and south-central Alaska, and results in a well-documented sequence of vegetation succession (Lutz 1956, Viereck 1973, Viereck and Schandelmeier 1980, Foote 1983, Van Cleve et al. 1983). However, compilation of information on forest fire distribution by the Alaska Fire Service revealed no major fires have occurred on Fort Richardson since 1950, although numerous fires have been recorded in the MatanuskaSusitna Valley. Within the study area, there is very low occurrence of early-successional ecotypes related to fire, such as Upland Rocky Moist Meadows (too little to map) and Upland Rocky Moist Tall Scrub (0.5\% area). In contrast, mid-successional ecotypes (i.e., Upland Rocky Moist Broadleaf Forest, Upland Rocky Moist Mixed Forests, Lowland Rocky Moist Broadleaf Forest, Lowland Rocky Moist Mixed Forests) occupy $\sim 47 \%$ of the study area. Late successional types (Upland and Lowland Rocky Moist Needleleaf Forests) occupy $\sim 4 \%$ of the area. This distribution of successional stages indicates that fires were prevalent in the 1800 s and early 1900 s and had a strong effect on ecosystem development, but have been nearly eliminated since 1950 by fire suppression associated with urban development.

The effects of fire on ecosystem development depend on the characteristics of the ecosystem (i.e., plant species, soils), and the severity and frequency of fires (Viereck 1973, Van Cleve et al. 1983). Regeneration pathways depend strongly on the degree to which the organic matter on the forest floor is burned, which in turn depends on fire severity. In general, forest stands are replaced by the same tree species (Viereck 1973, Van Cleve et al. 1983). On moist upland sites in interior Alaska, Foote (1983) identified six distinct successional stages: (1) newly burned stage (0-3 years); (2) herb-tree stage when fast growing mosses, herbs, and tree seedlings become established (3-10 years); (3) tall shrub-sapling stage (3-30 years; (4) dense tree stage of mostly birch, aspen, but also some white spruce (1530 years); (5) mature hardwood stage with quaking aspen and paper birch (50-150 years); and (6) spruce stage after 100-200 years.

Successional development of forests in south-central Alaska, however, can vary from this classical sequence. Marler and Vankat (1997) found that the oldest forests on Fort Richardson generally were mixed spruce-hardwood stands that reverted back to birch forests as the spruce trees became old or decadent. Successional relationships in south-central Alaska can be further complicated by outbreaks of spruce bark beetle (Dendroctonus rufipennis).

Insect outbreaks. Spruce bark beetles are one of the most important disturbance agents in mature white spruce stands in south-central and interior Alaska, although insect damage is more selective than other physical disturbances and affects only the tree canopy (Wittwer 2000). Statewide, 5.1 million acres were damaged by insects during 1994-1999, of which 2.2 million were damaged by spruce beetles. Spruce beetle activity peaked in 1996 at 1.1 million acres statewide and decreased to 0.6 million acres by 1999. In the Anchorage area, rates of beetle infestation also were highest in 1996 and 1997, but levels have substantially dropped since then because nearly all available host material has been killed.

The extent of the beetle infestation presented a problem for field classification and mapping because the mortality of spruce altered the forest classification. During fieldwork we noted dead spruce as a separate category, so that we could relate field classes with vegetation signatures on older aerial photography. The 1997 photography used for the mapping was taken at the height of bark beetle activity, and the amount of spruce in the canopy has decreased substantially since then. Our mapping and classification thus represent conditions in 1997. With the exception of increased deadfall, little is known about how damage to the spruce canopy alters the structure and function of the remaining community.

Fluvial processes. Channel migration associated with glacial and non-glacial rivers is a prominent process affecting the landscape on Fort Richardson, but the total area of the associated ecotypes is relatively small. Water in Rivers and Streams occupied $0.3 \%$ of the area, and Riverine Gravelly Moist Barrens covered $<0.1 \%$. Early- (scrub types, $0.2 \%$ of area), mid- (broadleaf and mixed forests, $1.2 \%$ of the area), and late(needleleaf forests, $0.2 \%$ ) successional ecotypes that have developed in response to channel migration occupied a total of only $1.6 \%$ of the landscape.

Studies in interior Alaska have described a charac- 
teristic pattern of vegetation succession along riverbanks (Drury 1956, Viereck 1970, Viereck et al. 1993 ) that is also somewhat applicable to south-central Alaska (Helm and Collins 1997). This successional sequence was described from meandering rivers with large, well-developed bars and contains stages that may be missing or very short-lived on higher energy streams. Generally, these models of floodplain succession indicate that (1) plant colonization is initiated by willows (0-5 years after establishment) when sufficient sediments have accumulated along the active channels, (2) a willow-alder stage occurs between 5 and 10 years after establishment, (3) forest stands develop through overstory dominance by poplar (20-100 years), (4) mixed stands of poplar and white spruce (100-200 years) develop, (5) mature white spruce (200-300 years) replaces those stands, and (6) black spruce ( $>500$ years) eventually becomes dominant (Viereck et al. 1993). The principal factors affecting this successional pattern are (1) decreasing sedimentation and water-table levels due to increasing bank height, (2) accumulation of organic matter from litter and (later) mosses, (3) burial of organic layers by flooding (provides the characteristic soil sequence of interbedded organics), and (4) the development of permafrost in interior and northern Alaska as soils become insulated by the thick organic layer (Van Cleve et al. 1993). Viereck et al. (1993) concluded that life-history characteristics and flooding events are the most important factors during the early stages of succession, whereas biological controls such as organic matter accumulation and competition become more important in middle and late stages.

Landslides and rockfalls. Slope instability in rugged mountainous areas and along steep bluffs has resulted in extensive areas of Alpine Rocky Dry Barrens within the study area (4.3\%). Little is known, however, about natural colonization and ecological development on this type of disturbed area. Other minor sources of instability and disturbance are landslides and slumps along bluffs being undercut by channel migration. Upland Rocky Dry Barrens associated with these areas occupy $0.1 \%$ of the area.

Coastal flooding. Tidally influenced coastal areas on Fort Richardson (3.6\% of total area) are limited to the Eagle River Flats and narrow beaches along Knik Arm. Coastal areas are some of the most dynamic ecosystems in Alaska because tidal flooding causes frequent inundation, sedimentation, and scouring, and creates a strong salinity gradient from frequently to infrequently flooded areas (Vince and Snow 1984, Kincheloe and Stehn 1991, Jorgenson 2000, Jorgenson and Ely 2001). The mean tidal range for Knik Arm is 9-11 $\mathrm{m}$ and occasional storms surges are much higher (Lawson et al. 1995). As a result of the high tidal range and large input of glacial sediments into Knik Arm, sedimentation rates range from $\sim 3 \mathrm{~mm} /$ year on levees to $10-15 \mathrm{~mm} /$ year on tidal flats, and $20-40 \mathrm{~mm} /$ year in tidal ponds (Lawson et al. 1995). These high sedimentation rates create extensive areas of Coastal Loamy Wet Barrens (1.1\% of area) that are occupied by pioneering vegetation adapted to frequent disturbance. Lateral erosion rates along tidal channels also are relatively high, ranging from $0.1 \mathrm{~m}$ to $9.8 \mathrm{~m}$ during the summers in 1992 and 1993 (Lawson et al. 1995). Steep salinity gradients from the nearshore water to the landward margin of the flats strongly controls the distribution of salt-tolerant species. Finally, coastal ecosystems along Cook Inlet have been affected by regional processes such as earthquakes, tectonic activity, and rebound after the last glaciation that have caused the surface to uplift or subside over time (Combellick 1994).

Thermokarst. Permafrost occurs in only a very small portion $(<0.1 \%)$ of the study area, primarily in patches of Lowland Needleleaf Forest Bog near Muldoon Road. In this area, there were numerous small collapse scars with Lowland Bog Meadow that have developed in response to thermokarst in the Lowland Needleleaf Forest Bog. Because frost-susceptible soils form only a thin $(\sim 1 \mathrm{~m})$ layer over gravel, thaw settlement is relatively minor $(<0.5 \mathrm{~m})$. Permafrost probably was much more widespread in the study area during the Little Ice Age, as indicated by the presence of small mounds in numerous lowland areas that appeared similar to thermokarst topography in interior Alaska. In contrast with the Anchorage area, permafrost degradation has been found to be widespread in interior Alaska and a significant ecological factor (Osterkamp et al. 2000, Jorgenson et al. 2001). Permafrost still persists at high elevations in the study area, as indicated by the presence of rock glaciers.

Human activity. Human-caused disturbances include cut-and-fill associated with construction of roads and pads, land clearing, gravel mining, ditching, trail development, munitions testing and training, and contaminants (Table 16, Fig. 27). Human-modified ecotypes occupied a large proportion of the study area (12.9\% of area), second only to fire-influenced ecotypes (described above). The most widespread human-disturbed types were Clearings With No Soil Removal (3.8\%), Clearings With Soil Removal (2.7\%), Clearings For Habitat Enhancement (2.2\%), and Disturbance Complex (2.1\%), which included roads, structures, and clearings primarily around the cantonment area.

Little is known about the response of subarctic ecosystems to disturbance because most disturbance research in Alaska has focused on tundra ecosystems (Van Cleve 1977, Walker et al. 1987, Slaughter et al. 1990). We evaluated differences in soil and vegetation char- 


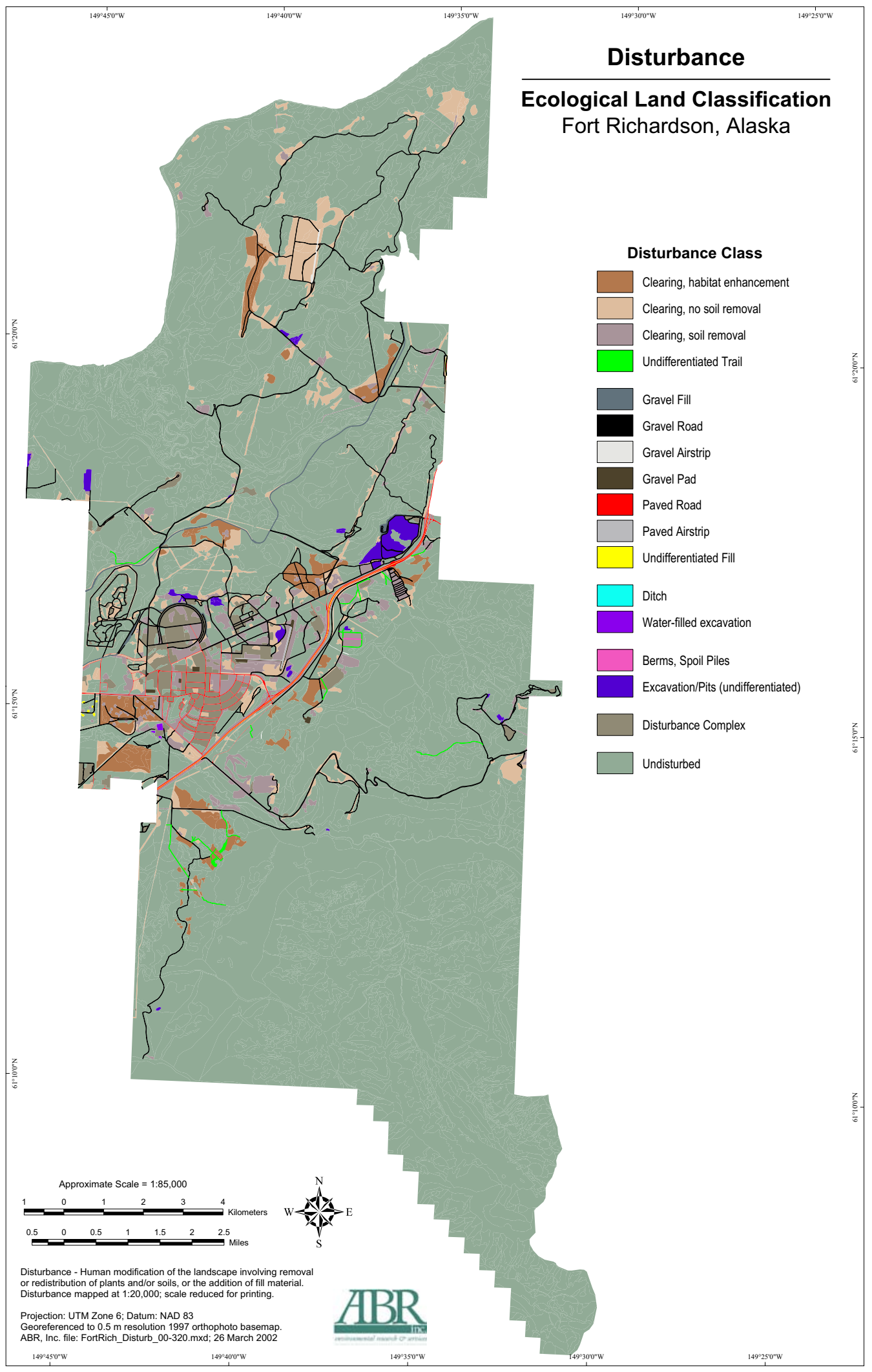

Figure 27. Map of human disturbances on Fort Richardson, south-central Alaska, 2001. 
acteristics among some of the dominant disturbance types on Fort Richardson in a separate report (Jorgenson et al. 2002) and we refer the reader to that report for more complete analysis. Below we provide brief descriptions of the types of human disturbances and refer to some of the pertinent literature.

The effects of roads on forest ecosystems have been assessed briefly by Brown and Berg (1980), but major studies on ecological effects of roads are lacking. In addition to the direct impact of loss of habitat by road construction, indirect impacts from dust and impoundments can be significant (Walker and Everett 1987).

Trails resulting from training exercises and recreational activities are common on Fort Richardson, but little is known about the ecological changes and recovery potential associated with trails in boreal ecosystems (Sparrow et al. 1978, Racine and Ahlstrand 1991). Generalization of the ecological effects and recovery potential is made difficult by the complex interactions of factors including ecosystem characteristics, seasonality of impacts, number of passes, and type of traffic.

A wide range of contaminants has been found on Fort Richardson, principally petroleum products and other organic compounds. Most of the known contaminated sites are located in the main cantonment area, and are associated with leakage at buildings, tank farms, landfills, fire-training pits, and drum burial sites. Another prominent contaminant is white phosphorus associated with explosives used in the impact areas, particularly on the Eagle River Flats. Waterfowl mortality due to white phosphorus contamination on the Eagle River Flats emerged as a significant natural resources issue in the early 1990s. A series of intensive evaluations and remedial investigations (Racine et al. 1993, Lawson et al. 1996) followed, resulting in placement of Fort Richardson on the National Priorities List by the EPA in 1994. Contaminated areas were not mapped in this study and the ecological effects of contaminants in the study area are still poorly understood.

In summary, fires have affected more area than any other disturbance type ( $48 \%$ of area over $\sim 200$ years), based on the occurrence of early to mid-successional forest stages that have developed since fires in the 1800s and early 1900s. Spruce beetle damage has been widespread in mixed forests and needleleaf forests, but the extent of the damage is difficult to quantify because beetles usually kill only a portion of the spruce trees in an area, and do not directly affect other species. Human activities have affected $13 \%$ of the area over $\sim 60$ years, of which $7.1 \%$ of the total area has recovered to shrublands and forests. In addition, some fires probably were caused by human activity. In contrast, disturbance due to channel migration has been negligible ( $1.6 \%$ over 200 years), as indicated by the occurrence of early to mid-successional stages that develop along riverbars. Disturbances associated with slope instability in alpine areas affected $4.3 \%$ of the study areas, as indicated by the occurrence of Alpine Rocky Dry Barrens. Areas highly disturbed by coastal flooding and sedimentation occupied only $1.1 \%$ of the area, as indicated by the occurrence of Coastal Loamy Wet Barrens. Finally, effects of thermokarst have been negligible $(<0.1 \%$ of area over $\sim 200-300$ years $)$, as indicated by the occurrence of thermokarst depressions associated with collapse scar bogs.

\section{Ecosections}

Based on differences in physiography, geomorphology, and soil texture, and on ecological relationships identified from field data, the 51 ecotypes were aggregated into 16 ecosections (Fig. 28, Table 17). These ecosections provide a simpler organizational framework for partitioning the variation in physical processes and biological characteristics. Usually, ecosections grouped ecotypes that are closely related successional stages that develop after a disturbance, such as fire, or that have slight differences in vegetation and soils related to slope position.

The dominant ecosections were Rocky Upland (43.0\% of area), Gravelly Lowland (18.9\%), and Rocky Alpine (17.9\%) (Table 18). Rocky Uplands were composed of Older Moraines, Kames (including kame-terraces), and Hillslope Colluvium deposits, which have similar well- to excessively drained soils. These rocky soils support tall scrub, and broadleaf, mixed, and needleleaf forests that represent closely related successional stages that develop after fire (Table 8). Gravelly Lowlands are similar to Rocky Uplands, but occur on flat, low-lying areas comprised of Alluvial Plain, Old Alluvial Fans, Meander Abandoned Channels, Alluvial Terraces, and Glaciofluvial Channels. Gravelly Lowlands have soils and vegetation similar to those of Rocky Uplands. Gravelly Lowlands were differentiated from Loamy and Peat-Rich Lowlands because the latter ecosections have poorly drained soils that support slower growing bog vegetation. The Rocky Alpine ecosection occurs above treeline and is composed of Bedrock, Hillslope Colluvium, Talus Cones, Younger and Older Moraine, and Solifluction deposits. The wellto excessively drained soils in this ecosection supported dwarf and low shrub vegetation, or frequently were barren due to movement of steep, unstable slopes. The remaining ecosections with widely varying characteristics covered relatively small areas.

The ecosection map offers several advantages for management applications. First, it greatly reduces the number of ecological map classes from 46 to 16 and thereby provides a much simpler framework for analy- 


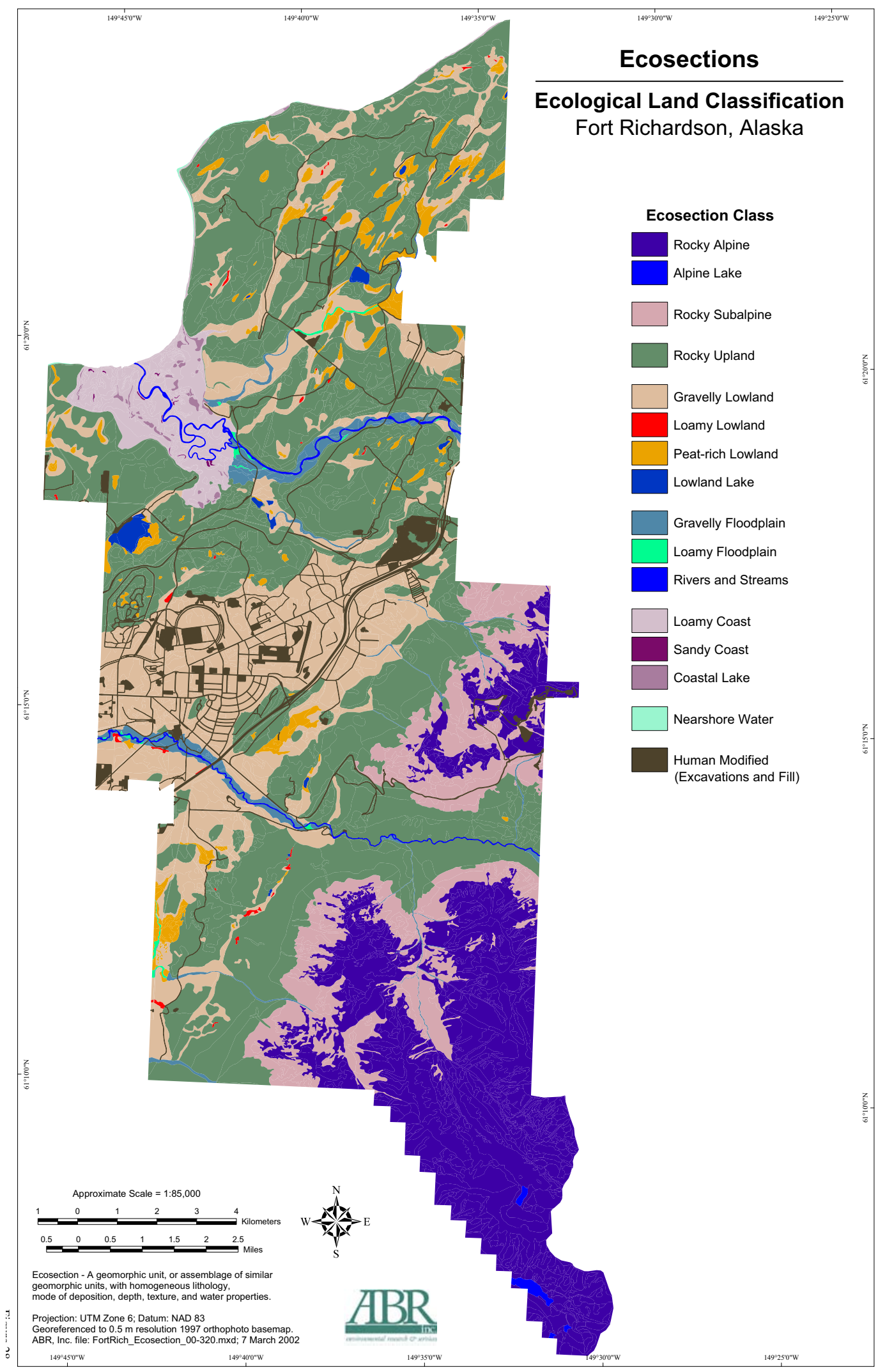

Figure 28. Map of ecosections based on geomorphic units for Fort Richardson, southcentral Alaska, 2001. 
Table 17. Classification and description of ecosections within Fort Richardson, south-central Alaska, 2001. Ecosections differentiate areas of uniform soil within a physiographic region; see landscape relationship table (Table 8) for associated ecotypes.

\begin{tabular}{|c|c|}
\hline Class & Description \\
\hline $\begin{array}{l}\text { Rocky } \\
\text { Alpine }\end{array}$ & $\begin{array}{l}\text { Steep to gentle slopes above treeline }(\sim 700 \mathrm{~m}) \text { comprised of metamorphic bedrock, talus cones, hillside } \\
\text { colluvium, and older and young moraine deposits. The soils range from well- to excessively drained soils } \\
\text { on upper slopes and crests with little to no organic horizon development, to moderately well-drained soils } \\
\text { with moderately thick organic horizons in snowbeds and along headwater streams. Dominant vegetation } \\
\text { types (Viereck Level IV) include Barrens in unstable exposed areas, Dryas-Lichen Tundra and Crowberry } \\
\text { Tundra in less-exposed areas, Cassiope Tundra in snowbeds, and Open Low Willow in more protected slopes } \\
\text { and drainages. }\end{array}$ \\
\hline $\begin{array}{l}\text { Alpine } \\
\text { Lake }\end{array}$ & Waterbodies above treeline generally lacking aquatic vascular vegetation. Water is deep $(>1.5 \mathrm{~m})$. \\
\hline $\begin{array}{l}\text { Rocky } \\
\text { Subalpine }\end{array}$ & $\begin{array}{l}\text { Upper slopes or drainages near treeline comprised of hillside colluvium, older moraine, solifluction, and } \\
\text { headwater floodplain deposits. Soils typically are rocky with a thin layer of organics, well drained, moist, } \\
\text { and strongly acidic. Dominant vegetation types include Closed Dwarf Mountain Hemlock and Open Balsam } \\
\text { Poplar in more protected areas downslope, Closed Tall Alder and Open Low Shrub Birch-Willow near } \\
\text { treeline, and Mixed Herbs on more moist concave slopes. }\end{array}$ \\
\hline $\begin{array}{l}\text { Rocky } \\
\text { Upland }\end{array}$ & $\begin{array}{l}\text { Well-drained slopes comprised of older moraine, kame, kame-terrace, drumlins, talus bluffs, and hillside } \\
\text { colluvium deposits. Soils typically are well-drained, moist, rocky or loamy with a moderately thin layer of } \\
\text { organics and very strongly acidic. Dominant vegetation types include Open White Spruce, Open } \\
\text { Spruce-Paper Birch, Open Paper Birch, and Closed Tall Scrub. }\end{array}$ \\
\hline $\begin{array}{l}\text { Gravelly } \\
\text { Lowland }\end{array}$ & $\begin{array}{l}\text { Flat, low-lying areas comprised of alluvial plain, meander floodplain abandoned channel, fluvial terrace, } \\
\text { and glaciofluvial channel deposits. Soils are gravelly, well- to somewhat well-drained, and very strongly } \\
\text { acidic with a thin organic and loam horizon. Dominant vegetation types include Open Black or White } \\
\text { Spruce, Open Spruce-Paper Birch, Closed Quaking Aspen-Spruce, and Open Paper Birch Forests. }\end{array}$ \\
\hline $\begin{array}{l}\text { Loamy } \\
\text { Lowland }\end{array}$ & $\begin{array}{l}\text { Flat low-lying areas or depressions comprised of glaciolacustrine, lacustrine, and meander floodplain } \\
\text { abandoned channel deposits. Soils are loamy with a thick organic layer, strongly acidic, and well to poorly } \\
\text { drained. Vegetation is dominated by Bluejoint Meadows. }\end{array}$ \\
\hline $\begin{array}{l}\text { Peat-rich } \\
\text { Lowland }\end{array}$ & $\begin{array}{l}\text { Flat low-lying areas or depression that have accumulated thick }(>40 \mathrm{~cm}) \text { organic deposits over lacustrine } \\
\text { and glaciolacustrine deposits. The organic soils are poorly to very poorly drained and very strongly acidic. } \\
\text { Vegetation is dominated by Open Black Spruce Forest, Open Dwarf Black Spruce Forest, Open Shrub } \\
\text { Birch-Ericaceous Shrub Bog, Open Low Sweetgale-Graminoid Bog, or Subarctic Lowland Sedge-Moss Bog } \\
\text { Meadow. }\end{array}$ \\
\hline $\begin{array}{l}\text { Lowland } \\
\text { Lake }\end{array}$ & $\begin{array}{l}\text { Deep lakes in kettles and basins partially vegetated }(5-30 \%) \text { with emergent or floating vegetation around } \\
\text { the edges. Water is strongly acidic and deep }(>1.5 \mathrm{~m}) \text {. Vegetation around the margins usually includes } \\
\text { Pondlilly Aquatic Herb. }\end{array}$ \\
\hline $\begin{array}{l}\text { Loamy } \\
\text { Floodplain }\end{array}$ & $\begin{array}{l}\text { Flat, low-lying areas on inactive channels, meander floodplain active overbank and meander floodplain } \\
\text { inactive overbank deposits with thick }(>40 \mathrm{~cm}) \text { deposits of fine-grained material at the surface. Soils are } \\
\text { loamy, wet, poorly drained with a thin organic layer and neutral pH. Vegetation is dominated by Open Tall } \\
\text { Alder. }\end{array}$ \\
\hline $\begin{array}{l}\text { Gravelly } \\
\text { Floodplain }\end{array}$ & $\begin{array}{l}\text { Flat areas on meander floodplain active riverbed, meander floodplain active overbank, meander floodplain } \\
\text { active overbank, and headwater floodplain deposits. Soils are moist, gravelly to sandy with a thin organic } \\
\text { layer, well drained, and moderately acidic to circum-neutral. Vegetation is dominated by barren areas on } \\
\text { gravel bars, Closed Tall Alder on active floodplains, and Open Black Cottonwood, Open Black } \\
\text { Cottonwood-White Spruce, and Open White Spruce Forests on inactive floodplains. }\end{array}$ \\
\hline $\begin{array}{l}\text { Rivers and } \\
\text { Streams }\end{array}$ & $\begin{array}{l}\text { Upper and lower perennial rivers and headwater streams from non-glacial and glacial sources. Water flows } \\
\text { year round in deep channels with places of riffles or shallow runs. Water is neutral to slightly alkaline. }\end{array}$ \\
\hline $\begin{array}{l}\text { Loamy } \\
\text { Coast }\end{array}$ & $\begin{array}{l}\text { Flat, low-lying coastal areas with loamy soils associated with active and inactive tidal flats. Soils usually } \\
\text { are poorly drained and brackish. Due to frequent flooding and sedimentation, much of the area is partially } \\
\text { vegetated. Vegetated areas are dominated by Halophytic Herb Wet Meadow, Halophytic Grass Wet Meadow, } \\
\text { Halophytic Sedge Marsh, and Open Low Sweetgale-Graminoid Shrub Meadow. }\end{array}$ \\
\hline $\begin{array}{l}\text { Sandy } \\
\text { Coast }\end{array}$ & $\begin{array}{l}\text { Flat, low-lying coastal areas with sandy soils on levees that develop along tidal guts. Soils are moderately } \\
\text { well drained and slightly brackish. Vegetation is dominated by Elymus meadows. }\end{array}$ \\
\hline $\begin{array}{l}\text { Coastal } \\
\text { Lake }\end{array}$ & $\begin{array}{l}\text { Brackish to slightly brackish shallow ponds on active and inactive tidal flats. Emergent or submergent } \\
\text { vegetation is common. Dominant vegetation types includes Halophytic Sedge Marsh and Aquatic Herb. }\end{array}$ \\
\hline $\begin{array}{l}\text { Nearshore } \\
\text { Water }\end{array}$ & Marine water in the nearshore zone. \\
\hline $\begin{array}{l}\text { Human } \\
\text { Modified }\end{array}$ & $\begin{array}{l}\text { Land that has been highly modified by human activity, such as roads, fill, and excavations. Areas where } \\
\text { vegetation has been modified but soils are relatively undisturbed are not included in this ecosection. }\end{array}$ \\
\hline
\end{tabular}


Table 18. Areal extent of ecosections mapped on Fort Richardson, south-central Alaska, 2001.

\begin{tabular}{lrrr}
\hline & \multicolumn{3}{c}{ Area } \\
\cline { 2 - 4 } Ecosection & acre & ha & $\%$ \\
\hline Rocky Alpine & 11,110 & 4496 & 17.9 \\
Alpine Lake & 91 & 37 & 0.1 \\
Rocky Subalpine & 5371 & 2174 & 8.7 \\
Rocky Upland & 26,660 & 10,789 & 43.0 \\
Gravelly Lowland & 11,697 & 4734 & 18.9 \\
Loamy Lowland & 77 & 31 & 0.1 \\
Peat-rich Lowland & 1508 & 610 & 2.4 \\
Lowland Lake & 235 & 95 & 0.4 \\
Loamy Floodplain & 89 & 36 & 0.1 \\
Gravelly Floodplain & 1126 & 456 & 1.8 \\
Rivers and Streams & 169 & 68 & 0.3 \\
Loamy Coast & 2042 & 826 & 3.3 \\
Sandy Coast & 16 & 6 & $<0.1$ \\
Coastal Lake & 122 & 50 & 0.2 \\
Nearshore Water & 26 & 11 & $<0.1$ \\
Human Modified & 1656 & 670 & 2.7 \\
Total & 61,996 & 25,089 & 100 \\
\hline
\end{tabular}

sis and management. Second, it groups ecotypes with similar physical and floristic characteristics. For example, the five ecotypes that comprised the rocky alpine ecosection have relatively similar species composition (Table 11). Third, different ecotypes within an ecosection are likely to have similar responses to disturbance. For example, terrain disturbances on Rocky Uplands, such as fire or land clearing, are likely to have vegetation that progresses through similar successional sequences from herbaceous meadows and tall shrub to forest vegetation.

\section{Ecodistricts}

Ecodistricts provide a regional approach to stratifying the landscape based on recurring patterns of geomorphic classes with distinctive physiographic characteristics. Ecosubdistricts are similar, but delineate smaller areas with less variability in geomorphic units. Fort Richardson, extending from Cook Inlet to the Chugach Mountains, crosses four ecodistricts and eight ecosubdistricts (Fig. 29, Table 19). Six other ecosubdistricts also were mapped immediately adjacent to the study area.

The ecodistricts and ecosubdistricts provide a way of grouping the distribution of ecotypes that usually are contextually related on the landscape. For example, rocky alpine ecotypes were found only in the Eklutna Mountains ecosubdistrict, because they are associated with high elevations and rugged topography (Fig. 15). Lowland Gravelly Mixed Forests, Lowland Needleleaf Forests, Lowland Scrub Bog, and Lowland Bog Meadows were widespread in the Anchorage Glaciated Lowlands (Fig. 12), while Upland Rocky Moist Broadleaf Forests and Upland Rocky Moist Mixed Forests were common in the Knik Glaciated Lowlands (Fig. 13) and the Anchorage Glaciated Hillsides (Fig. 14). Lowland Lakes and Marshes were found almost exclusively in the Knik Glaciated Lowlands. Halophytic ecotypes are restricted to the Upper Cook Inlet Coast (Fig. 10).

This successive partitioning of the landscape is not only useful for stratifying field sampling, but improves the reliability of conceptual models of ecosystem distribution developed from toposequences because vegetation and soil patterns often vary among ecodistricts. In turn, the ecodistricts are useful for land management, because management concerns and objectives will be different, depending on the predominant geomorphic and vegetation characteristics of the area.

\section{SUMMARY AND CONCLUSIONS}

An ecological land survey (ELS) of Fort Richardson land was conducted; ecosystems were mapped at three spatial scales to aid in the management of natural resources. In an ELS, landscapes are viewed not just as 




Figure 29. Map of ecodistricts and ecosubdistricts in the vicinity of Fort Richardson, south-central Alaska, 2001. 
Table 19. Classification and description of ecosubdistricts in the vicinity of Fort Richardson, south-central Alaska, 2001. Ecosubdistricts are equivalent to subsections of ECOMAP (1993).

\begin{tabular}{lrl}
\hline & Ecosub- \\
Ecodistrict & district & Description \\
\hline
\end{tabular}

Gulf of Alaska Ecoregion

Cook Inlet

Marine water of the upper Cook Inlet north of Nikiski. Water has high sediment concentrations as the result of input of glacial meltwater and resuspension of sediments from extensive tidal flats. The inlet has high tidal range with semi-diurnal fluctuations of $9-11 \mathrm{~m}$. Ice flows are prevalent during the winter. Shallow waters present hazards to navigation.

Upper Cook Inlet Ecoregion

Cook Inlet Upper Cook Low-lying, salt-affected areas along the coast of Upper Cook Inlet. Geomorphologic Basin Inlet Coast

Ecoregion

units include active tidal flat deposits subject to frequent tidal inundation and sedimentation and inactive tidal flats where soils are slightly brackish with thin organic matter accumulations. Coastal areas have been affected by uplift and subsidence after earthquakes. Vegetated areas above mean higher high water are dominated by halophytic herb and grass wet meadows, halophytic sedge marsh, and sweetgale-graminoid shrub meadows.

Matanuska Susitna Flats

Lowlands

Flat, low-lying areas along the lower Susitna River affected by glaciomarine and glacioalluvial process. Surficial deposits include sandy alluvial fan and deltaic deposits, unpitted outwash plains near glacial moraines, and bog deposits. The area generally is underlain by silty clay of the Bootlegger Cove Formation. The area is affected by substantial surface and subsurface water movement creating extensive areas of bogs and fens. Vegetation of the area is dominated by open black spruce, shrub birch-ericaceous shrub bogs, subarctic lowland sedge - moss bog meadows, and Sphagnum bogs.

Knik Undulating portions of the Cook Inlet Basin in the lower Matanuska and Susitna valleys Glaciated and near Eagle River that have distinct kame and kettle topography associated with the Lowlands Elmendorf advance of the Naptowne glaciation during the late Wisconsin period $(13,500$ years BP). The Elmendorf advance deposited distinct lobes of glacial-related deposits including end moraines, ground moraines, drumlins, eskers, and crevasse-fill-ridge deposits. Upper slopes and crests have well-drained, acidic, rocky soils that support white spruce and birch forests. Lower slopes and depressions have poorly drained, acidic, organic soils that support black spruce forests, shrub birch-ericaceous shrub bogs, and subarctic lowland sedge - moss bog meadows. Kettle lakes are common.

Anchorage Flat to gently undulating terrain near Anchorage resulting from complex depositional Glaciated processes. Surficial deposits include the Bootlegger Cove Formation of silty clay Lowlands sediments deposited in a glaciomarine environment, glaciodeltaic deposits near Point Campbell and Fire Island, extensive glaciofluvial deposits associated with outwash from the Elmendorf moraine, old alluvial fans extending from the Chugach mountains, modern fluvial deposits along rivers and streams, and extensive bog deposits. Vegetation and soils are similar to those described for the Knik Glaciated Lowlands, except bogs are more extensive.

Matanuska - Highly dynamic braided floodplains of the Matanuska and Knik Rivers that receive high Knik Floodplain seasonal discharge from melting glaciers during late summer. Geomorphic units include lower perennial river, braided active channel deposits, braided active overbank deposits, and braided inactive overbank deposits. Soils are highly variable in texture from loamy to rocky, well-drained, and circum-neutral. Vegetation is dominated by early successional herbs on gravel bars, alder-willow scrub on active overbank deposits, and black cottonwood and white spruce on higher inactive cover deposits.

Matanuska- Flat, low-lying floodplains with small rivers within the Matanuska-Susitna Lowlands

Susitna

Lowland Rivers can be either glacial or non-glacial, and the adjacent floodplains are subject to occasional flooding and sedimentation. Geomorphic units include lower perennial river, Floodplains meander active channel deposits, meander active overbank deposits, and meander inactive overbank deposits. Soils are highly variable in texture from loamy to rocky, well-drained, and circum-neutral. Vegetation is dominated by early successional herbs on gravel bars, alder - willow scrub on active floodplains, and black cottonwood and white spruce on higher inactive floodplains. 
Table 19 (cont'd).

\begin{tabular}{|c|c|c|}
\hline Ecodistrict & $\begin{array}{c}\text { Ecosub- } \\
\text { district }\end{array}$ & Description \\
\hline \multicolumn{2}{|c|}{ Chugach-St. Elias Mountains } & Ecoregion \\
\hline \multirow[t]{3}{*}{$\begin{array}{l}\text { Northern } \\
\text { Chugach } \\
\text { Mountains }\end{array}$} & $\begin{array}{l}\text { Eklutna } \\
\text { Mountains }\end{array}$ & $\begin{array}{l}\text { Rugged, mostly non-glaciated portion of the Chugach Mountains extending along the } \\
\text { north side of the mountains from Turnagain Arm to the Chitina River. The northern side } \\
\text { of the mountains has a more continental, colder, and drier climate, than the coastal } \\
\text { mountains. Bedrock is comprised mostly of metamorphic rocks including } \\
\text { metasandstone, greenstones, metachert, argillite, graywacke, and phyllite. Higher } \\
\text { mountain peaks have barren fellfields and occasional glaciers; hillslopes have } \\
\text { colluvium with well-drained rocky soils. Vegetation above treeline is dominated by } \\
\text { dryas tundra, crowberry tundra, and cassiope tundra. More protected slopes have } \\
\text { spruce-birch forests and alder scrub. }\end{array}$ \\
\hline & $\begin{array}{l}\text { Eklutna } \\
\text { Mountain } \\
\text { Hillsides }\end{array}$ & $\begin{array}{l}\text { Moderately sloping hillside and U-shaped valleys along the western front of the } \\
\text { Northern Chugach Mountains. Climate on the northern side of the mountains is more } \\
\text { continental with colder winter temperatures and less precipitation. The area has been } \\
\text { affected by the Naptowne and Knik glaciations of the late Pleistocene. Surficial deposits } \\
\text { on the hillsides include lateral moraines, kame terraces, glaciofluvial channel, hillslope } \\
\text { colluvium, landslide, and occasional bog deposits. Soils generally are rocky with thin } \\
\text { organic horizons, well-drained, and acidic. Vegetation is dominated by spruce-birch } \\
\text { forests, and alder scrub. Sitka spruce and western hemlock, which are at the northernmost } \\
\text { extent of their distribution, still occur in this area. }\end{array}$ \\
\hline & $\begin{array}{l}\text { Northern } \\
\text { Chugach } \\
\text { Floodplains }\end{array}$ & $\begin{array}{l}\text { Gently to moderately sloping floodplains of braided and meandering rivers in the } \\
\text { Chugach Mountains. Rivers can be either glacial or non-glacial and the adjacent } \\
\text { floodplains are subject to occasional flooding and sedimentation. Geomorphic units } \\
\text { include lower perennial river, active channel deposits, active overbank deposits, and } \\
\text { inactive overbank deposits. Soils are highly variable in texture from loamy to rocky, } \\
\text { well-drained, and circum-neutral. Vegetation is dominated by early successional herbs } \\
\text { on gravel bars, alder-willow scrub on active overbank deposits, and black cottonwood } \\
\text { and white spruce on higher inactive cover deposits. }\end{array}$ \\
\hline $\begin{array}{l}\text { Southern } \\
\text { Chugach } \\
\text { Mountains }\end{array}$ & $\begin{array}{l}\text { Chugach Ice } \\
\text { Fields }\end{array}$ & $\begin{array}{l}\text { Rugged ice-clad coastal mountains surrounding Prince William Sound. Huge snow and } \\
\text { ice fields occasionally are interrupted by rock cliffs and small exposed peaks. In the } \\
\text { summer, high volumes of meltwater discharge from the ice fields and glaciers and create } \\
\text { abundant waterfalls over high cliffs and feed large glacial rivers. Thin and rocky soils } \\
\text { exist where mountain summits and slopes are devoid of ice, snow, and active scree. While } \\
\text { most non-glaciated areas are barren, vegetated areas commonly have dryas tundra, } \\
\text { crowberry tundra, and cassiope tundra, and alder shrublands. }\end{array}$ \\
\hline \multirow[t]{2}{*}{$\begin{array}{l}\text { Kenai } \\
\text { Mountains }\end{array}$} & $\begin{array}{l}\text { Inner } \\
\text { Turnagain } \\
\text { Coast }\end{array}$ & $\begin{array}{l}\text { Low-lying salt-affected areas along the coast of Turnagain Arm, situated within the } \\
\text { Chugach-St. Elias Mountains ecoregion. Geomorphology ranges from active tidal flat } \\
\text { deposits subject to frequent tidal inundation and sedimentation to inactive tidal flats } \\
\text { where soils are slightly brackish and organic matter can accumulate. The coastal areas } \\
\text { have been affected by uplift and subsidence after earthquakes, and to rebound after } \\
\text { deglaciation. Vegetated areas above the barren mudflats are dominated by halophytic } \\
\text { herb and sedge wet meadows, halophytic sedge marsh, and sweetgale-graminoid shrub } \\
\text { meadows. }\end{array}$ \\
\hline & $\begin{array}{l}\text { Turnagain } \\
\text { Arm Valleys }\end{array}$ & $\begin{array}{l}\text { Gentle to steep slopes in U-shaped valleys in the Chugach Mountains along Turnagain } \\
\text { Arm. The areas are subject to heavy snowfall and avalanches are prevalent. } \\
\text { Geomorphology includes hillside colluvium, landslide deposits, glacial moraines, and } \\
\text { headwater floodplains. Soils range from well-drained in steep rocky areas to poorly } \\
\text { drained on toe slopes. Vegetation is dominated by Sitka spruce forests with thick moss } \\
\text { accumulations on the ground and on the trees. Alder scrub is common in avalanche } \\
\text { tracks. }\end{array}$ \\
\hline
\end{tabular}


aggregations of separate biological and earth resources, but as ecological systems with functionally related parts that can provide a consistent conceptual framework for modeling, analyzing, interpreting, and applying ecological knowledge. Land management activities such as ecological risk assessments, analysis and mapping of terrain sensitivity, protection and enhancement of wetlands and wildlife habitats, planning for training exercises, and fire management all require spatially explicit information and a method of organizing ecological information. To provide the information required for such a wide range of applications, an ELS involves three types of effort: (1) an ecological land survey that inventories and analyzes data obtained in the field, (2) an ecological land classification that classifies and maps ecosystem distribution, and (3) an ecological land evaluation that assesses the capabilities of the land for various land management practices.

Field surveys at 132 plots along 16 toposequences and at 99 other plots were used to develop a better understanding of the ecological processes controlling landscape development in the study area. Co-varying relationships among physiography, geomorphology, macrotopography, hydrology, and vegetation were identified using field survey data. The relationships revealed that the various ecosystem components were closely related to fire effects and geomorphic processes, such as floodplain development, landslide and slope instability, and coastal flooding. Association of vegetation structures (e.g., closed deciduous forests) with geomorphic units (e.g., inactive cover deposits) were used to identify 51 ecotypes (local ecosystems) that were effective at differentiating dominant species (e.g., balsam poplar in riverine forests versus paper birch in upland forests) and plant associations.

To facilitate development of an ecological classification, information on individual landscape components was compiled and an integrated-terrain-unit approach was used to synthesize this information during mapping. Climate data indicates the area is transitional between the colder continental climate of the boreal region of interior Alaska and the warmer maritime climate of southern coastal Alaska. Bedrock geology is dominated by a complex of metamorphic rocks in the Northern Chugach Mountains and quaternary deposits in the lowlands. Highly detailed mapping of surficial geology by previous investigations was compiled and simplified into a reduced set of 39 geomorphic units more appropriate for ecological mapping. Topographic characteristics were classified into 24 surface forms that covered the entire elevation gradient from the coast to Tanaina Peak (1615 m, $5300 \mathrm{ft})$. The Alaska Vegetation Classification system was used to differentiate 46 vegetation classes and five nonvegetated classes. Geomorphology, surface forms, and vegetation were mapped using an integrated-terrain-unit approach that included multiple coding of terrain components for each land unit. The mapping created a standard set of linework for all components registered to a highly controlled orthophoto mosaic developed from 1997 aerial photography. This map and geodatabase were used to develop ecological maps that integrated physical and biological characteristics of the landscape.

Ecosystem maps were developed at three spatial scales. Forty-six ecotypes (1:20,000 scale) derived from the ITU mapping, differentiated areas with homogenous topography, terrain, soil, surface-form, hydrology, and vegetation. Vegetation (structure and composition) and environmental (elevation, organic matter accumulation, depth to rock, water depths, $\mathrm{pH}$, and electrical conductivity) characteristics of ecotypes were summarized using data obtained from field surveys. Sixteen ecosections (1:100,000 scale) were aggregated from the ecotypes to differentiate areas that are homogeneous with respect to geomorphic features and soil texture, and thus have recurring patterns of soils and vegetation. The ecotypes within an ecosection usually represented different stages in a single successional sequence. Four ecodistricts and eight ecosubdistricts $(1: 250,000)$ were developed from separate mapping of Landsat imagery to differentiate broader areas with similar physiography, geology, and geomorphology.

This spatial database can now become the foundation for numerous management tasks including wetland protection, integrated training-area management, wildlife management, and recreational area management. The hierarchical approach, which incorporates multiple ecosystem components into general ecotypes, allows users to partition the variability of a wide range of ecological characteristics. In turn, this hierarchical linkage of ecological characteristics facilitates the production of specialized thematic maps (e.g., soil erosion hazard, timber types) based on recoding of the map database, and thus provides flexibility for addressing a wide range of management objectives. Finally, the database structure allows continued development of the spatial database within a geographic information system as the concepts of ecosystem management continue to evolve.

\section{LITERATURE CITED} Alaska Division of Geological and Geophysical Sur-
veys (ADGGS) (1983) Engineering geology mapping
classification system. Alaska Division of Geology and
Geophysical Surveys, Fairbanks, Alaska, unpublished
report, 76 p.
Allen, T.F.H., and T.B. Starr (1982) Hierarchy: Per-
spectives for Ecological Complexity. Chicago, Illinois: 
University of Chicago, $310 \mathrm{p}$.

Austin, M.P., and P.C. Heyligers (1989) Vegetation survey design for conservation: Gradsect sampling of forests in northeastern New South Wales. Biological Conservation, 50:13-32.

Bailey, R.G. (1980) Descriptions of ecoregions of the United States. U.S. Department of Agriculture, Washington, D.C., Miscellaneous Publication No. 1391.

Bailey, R.G. (1996) Ecosystem Geography. New York: Springer-Verlag, 199 p.

Bailey, R.G. (1998) Ecoregions map of North America explanatory note. U.S. Forest Service, Washington, D.C., Miscellaneous Publication 1548.

Barnes, B.V., K.S. Pregitzer, T.A. Spies, and V.H. Spooner (1982) Ecological forest site classification. Journal of Forestry, 80: 493-498.

Brinson, M.M. (1993) A hydrogeomorphic classification for wetlands. U.S. Army Engineer Waterways Experiment Station, Vicksburg, Mississippi, Technical Report WRP-DE-4.

Brown, J., and R.L. Berg (Ed.) (1980) Environmental engineering and ecological baseline investigations along the Yukon River-Prudhoe Bay Haul Road. U.S. Army Cold Regions Research and Engineering Laboratory, Hanover, New Hampshire, CRREL Report 8019.

Combellick, R.A. (1994) Investigations of peat stratigraphy in tidal marshes along Cook Inlet, Alaska, to determine the frequency of 1964-type great earthquakes in the Anchorage region. Alaska Division of Geological and Geophysical Surveys, Fairbanks, Alaska, Report of Investigations 94-7.

Davidson, D. (1997) Chugach National Forest: Ecosections and Sub-Sections. U.S. Forest Service, Anchorage, Alaska (http://agdc.usgs.gov/data/usfs/ chugach/ecosections.html).

Delcourt, H.R., and P.A. Delcourt (1988) Quaternary landscape ecology: Relevant scales in space and time. Landscape Ecology, 2: 23-44.

Driscoll, R.S., D.L. Merkel, D.L. Radloff, D.E. Snyder, and J.S. Hagihara (1984) An ecological land classification framework for the United States. U.S. Department of Agriculture, Washington, D.C., Miscellaneous Publication 1439.

Drury, W.H. (1956) Bog flats and physiographic processes in the upper Kuskokwim River region, Alaska. Contributions from the Gray Herbarium of Harvard University, No. CLXXVIII, 130 p.

ECOMAP (1993) National hierarchical framework of ecological units. U.S. Forest Service, Washington, D.C., $20 \mathrm{p}$.

Ellert, B.H., M.J. Clapperton, and D.W. Anderson (1997) An ecosystem perspective of soil quality. In Soil Quality for Crop Production and Ecosystem Health: Developments in Soil Science (E.G. Gregorich and M.R.
Carter, Ed.), p. 115-141. Amsterdam, Netherlands: Elsevier Science Publications.

Fitter, A.H., and R.K.M. Hay (1987) Environmental Physiology of Plants. San Diego, California: Academic Press, $423 \mathrm{p}$.

Foote, J.M. (1983) Classification, description, and dynamics of plant communities after fire in the taiga of Interior Alaska. Pacific Northwest Forest and Range Experiment Station, U.S. Forest Service, Portland, Oregon, Research Paper PNW-307, 108 p.

Forman, R.T. (1995) Land Mosaics: The Ecology of Landscapes and Regions. Cambridge, UK: Cambridge University Press.

Gallant, A.L., E.F. Binnian, J.M. Omernik, and M.B. Shasby (1995) Ecoregions of Alaska. U.S. Government Printing Office, Washington, D.C., U.S. Geological Survey Professional Paper 1567, 73 p.

Gossweiler, W.A. (1984) Fort Richardson Natural Resources Management Plan. Natural Resources Branch, Fort Richardson, unpublished report, $114 \mathrm{p}$.

Helm, D.J., and W.B. Collins (1997) Vegetation succession and disturbance on a boreal forest floodplain, Susitna River, Alaska. Canadian Field Naturalist, 111: 553-566.

Hulten, E. (1968) Flora of Alaska and Neighboring Territories. Stanford: Stanford University Press, 1008 p. Hunter, L.E., D.E. Lawson, S.R. Bigl, P.B. Robinson, and J.D. Schlagel (2000) Glacial geology and stratigraphy of Fort Richardson, Alaska: A review of available data on the hydrogeology. U.S. Army Engineer Research and Development Center, Cold Regions Research and Engineering Laboratory, Hanover, New Hampshire, ERDC/CRREL Technical Report TR-003, 68 p.

Ignatov, M.S., and O.M. Afonina (Ed.) (1992) Checklist of mosses of the former USSR. Arctoa, 1(1-2): 185.

Jenny, H. (1941) Factors of Soil Formation. New York: McGraw-Hill Book Co., 281 p.

Johnson, H.A., and H.T. Jorgenson (1963) The Land Resources of Alaska. New York: University Publishers, $551 \mathrm{p}$.

Jorgenson, M.T. (2000) Hierarchical organization of ecosystems at multiple spatial scales on the YukonKuskokwim Delta, Alaska. Arctic, Antarctic, and Alpine Research, 32: 221-239.

Jorgenson, M.T., and C. Ely (2001) Topography and flooding of coastal ecosystems on the YukonKuskokwim Delta: Implications for sea-level rise. Journal of Coastal Research, 17: 124-136.

Jorgenson, M.T., J.E. Roth, E.R. Pullman, R.M. Burgess, M. Raynolds, A.A. Stickney, M.D. Smith, and T. Zimmer (1997) An ecological land survey for the Colville River Delta, Alaska, 1996. Prepared for ARCO Alaska, Inc., Anchorage, Alaska, by ABR, Inc., 
Fairbanks, Alaska, unpublished report, 160 p.

Jorgenson, M.T., J. Roth, M. Raynolds, M.D. Smith, W. Lentz, A. Zusi-Cobb, and C.H. Racine (1999) An ecological land survey for Fort Wainwright, Alaska. U.S. Army Cold Regions Research and Engineering Laboratory, Hanover, New Hampshire, CRREL Report 99-9, 83 p.

Jorgenson, M.T., J.E. Roth, M.D. Smith, S. Schlentner, W. Lentz, and E.R. Pullman (2001) An ecological land survey for Fort Greely, Alaska. U.S. Army Engineer Research and Development Center, Cold Regions Research and Engineering Laboratory, Hanover, New Hampshire, ERDC/CRREL Technical Report TR-01-04, 85 p.

Jorgenson, M.T., J.E. Roth, E.R. Pullman, S.F. Schlentner, T. Schick, and M. Macander (2002) An ecological land evaluation for Fort Richardson, Alaska. Prepared for U.S. Army Alaska, Anchorage, Alaska, by ABR, Inc., Fairbanks, Alaska, unpublished report.

Kincheloe, K.L., and R.A. Stehn (1991) Vegetation patterns and environmental gradients in coastal meadows on the Yukon-Kuskokwim delta, Alaska. Canadian Journal of Botany, 69: 1616-1627.

Klijn, F., and H.A. Udo de Haes (1994) A hierarchical approach to ecosystem and its implication for ecological land classification. Landscape Ecology, 9: 89104.

Konsantinova, N.A., A.D. Potemkin, and R.N. Schljakov (1992) Check-list of the Hepaticae and Anthocerotae of the former USSR. Arctoa, 1(1-2): 87127.

Kreig, R.A., and R. D. Reger (1982) Air-photo analysis and summary of landform soil properties along the route of the Trans-Alaska Pipeline System. Alaska Division of Geological and Geophysical Surveys, Geologic Report 66, 149 p.

Lawson, D.E., S.R. Bigl, J. Bodette, and P. Weyrick (1995) Initial analysis of Eagle River Flats hydrology and sedimentology, Fort Richardson, Alaska. U.S. Army Cold Regions Research and Engineering Laboratory, Hanover, New Hampshire, CRREL Report 95-5, 39 p. Lawson, D.E., L.E. Hunter, S.R. Bigl, B.M. Nadeau, P.B. Weyrick, and J.H. Bodette (1996) Physical system dynamics and white phosphorus fate and transport, 1994, Eagle River Flats, Fort Richardson, Alaska. U.S. Army Cold Regions Research Engineering Laboratory, Hanover, New Hampshire, CRREL Report 96-9, 64 p. Levin, S.A. (1992) The problem of pattern and scale in ecology. Ecology, 73: 1943-1967.

Lichvar, R., C. Racine, B. Murray, J. Tande, R. Lipkin, and M. Duffy (1997) A floristic inventory of vascular and cryptogam plant species at Fort Richardson, Alaska. U.S. Army Corps of Engineers, Waterways Experiment Station, Vicksburg, Mississippi, Technical Report EL-97-4, 23 p.
Lutz, H.J. (1956) Ecological effects of forest fires on the vegetation of interior Alaska. U.S. Forest Service, Fairbanks, Alaska, Technical Bulletin 1133, 121 p.

Marler, S.C., and J.L. Vankat (1997) Old growth forests of Fort Richardson, Alaska: Composition, structure and development. Prepared for Environmental Resources Department, Fort Richardson, Alaska, by Miami University, Oxford, Ohio, unpublished report, $25 \mathrm{p}$.

Miall, A.D. (1985) Architectural-element analysis: A new method of facies analysis applied to fluvial deposits. Earth Sciences Review, 22: 261-308.

National Resources Conservation Service (NRCS) (2000) Soil inventory and mapping of Fort Richardson. U.S. Department of Agriculture, Anchorage, Alaska, unpublished report and maps.

National Resources Conservation Service (NRCS) (2001) The PLANTS Database, Version 3.1 (http:// plants.usda.gov). National Plant Data Center, Baton Rouge, Louisiana.

National Wetlands Working Group (NWWG) (1988) Wetlands of Canada. Environment Canada, Montreal, Quebec. Ecological Land Classification Series, No. 24, $452 \mathrm{p}$.

Nokleberg, W.J., and G. Plafker (1994) Geology of south-central Alaska. In The Geology of Alaska (G. Plafker and H.C. Berg, Ed.), p. 311-366. The Geological Society of America, Boulder, Colorado, volume G-1. Nowacki, G., P. Spencer, T. Brock, M. Fleming, and T. Jorgenson (2002) Ecoregions of Alaska and neighboring territories. U.S. Geological Survey, Washington, D.C. (ftp://agdcftp1.wr.usgs.gov/pub/projects/fhm/ akecoregions.jpg).

Oberbauer, S.F., S.J. Hastings, J.L. Beyers, and W.C. Oechel (1989) Comparative effects of downslope water and nutrient movement of plant nutrition, photosynthesis, and growth in Alaskan tundra. Holarctic Ecology, 12: 324-334.

O'Neil, R.V., D.L. DeAngelis, J.B. Waide, and T.F.H. Allen (1986) A hierarchical concept of ecosystems. Princeton University Press, Princeton, New Jersey.

Osterkamp, T.E., L. Viereck, Y. Shur, M.T. Jorgenson, C. Racine, A. Doyle, and R.D. Boone (2000) Observations of thermokarst and its impact on boreal forests in Alaska, U.S.A. Arctic, Antarctic and Alpine Research, 32(3): 303-315.

Pickett, S.T., J. Kolasa, J.J. Armesto, and S.L. Collins (1989) The ecological concept of disturbance and its expression at various hierarchical levels. Oikos, 54: 129-136.

Plafker, G., J.C. Moore, and G.R. Winkler (1994) Geology of the southern Alaska margin. In The Geology of Alaska (G. Plafker, and H.C. Berg, Ed.), p. 389449. The Geological Society of America, Boulder, Colorado, volume G-1. 
Quirk, W.A., W. Gossweiler, and E. Kiker (1978) Natural Resources Conservation Program: A management plan for natural resources on 172nd Infantry Brigade Lands, Alaska. Natural Resources Branch, Fort Richardson, unpublished report, $30 \mathrm{p}$.

Racine, C.H., and G.M. Ahlstrand (1991) Thaw response of tussock-shrub tundra to experimental all-terrain vehicle disturbance in south-central Alaska. Arctic, 44: 31-37.

Racine, C.H., M.E. Walsh, C.M. Collins, S. Taylor, B.D. Roebuck, L. Reitsma, and B. Steele (1993) White phosphorus contamination of salt marsh sediments at Eagle River Flats, Alaska. U.S. Army Cold Regions Research and Engineering Laboratory, Hanover, New Hampshire, CRREL Report 93-17.

Reger, R.D., and R.G. Updike (1983) Upper Cook Inlet Region and the Matanuska Valley. In Richardson and Glenn Highways, Alaska: Guidebook to Permafrost and Quaternary Geology (T.L. Péwé and R.D. Reger, Ed.), p. 185-259. Fairbanks, Alaska: Alaska Division of Geological and Geophysical Surveys, Guidebook 1.

Reger, R.D., R.A. Combellick, and J. BrighamGrette (1995) Late-Wisconsin events in the Upper Cook Inlet region, south-central Alaska. In Short Notes on Alaska Geology 1995 (R.A. and T.F. Combellick, Ed.), p. 33-45. Alaska Division of Geological and Geophysical Surveys, Fairbanks, Alaska, Professional Report 117D.

Rieger, S., D.B. Schoephorster, and C.E. Furbush (1979) Exploratory soil survey of Alaska. Soil Conservation Service, U.S. Department of Agriculture, Washington, D.C., $213 \mathrm{p}$.

Rowe, J.S. (1961) The level-of-integration concept and ecology. Ecology, 42: 420-427.

Schmoll, H.R., and E. Dobrovolny (1972) Generalized geologic map of Anchorage and vicinity, Alaska. U.S. Geological Survey, Washington, D.C. Miscellaneous Investigations Map I-787-A.

Schmoll, H.R., L.A Yehle, and E. Dobrovolny (1996) Surficial geologic map of the Anchorage A-8 NE quadrangle, Alaska. U.S. Geological Survey, Denver, Colorado, Open File Report 96-003.

Schoeneberger, P.L., P.A. Wysocki, E.C. Benham, and W.D. Broderson (1998) Fieldbook for describing and sampling soils. National Soil Survey Center, Natural Resource Conservation Service, U.S. Dept. of Agriculture, Lincoln, Nebraska.

Shugart, H.H. (1998) Terrestrial Ecosystems in Changing Environments. Cambridge, UK: Cambridge University Press.

Slaughter, C.W., C.H. Racine, D.A. Walker, L.A. Johnson, and G. Abele (1990) Use of off-road vehicles and mitigation of effects of Alaska permafrost environments: A review. Environmental Management, 14:
63-72.

Soil Survey Division Staff (SSDS) (1993) Soil Survey Manual. U.S. Department of Agriculture, Washington, D.C., Handbook No. 18, 437 p.

Soil Survey Staff (SSS) (1998) Keys to Soil Taxonomy, Eighth Edition. Washington, D.C.: U.S. Department of Agriculture.

Sparrow, S.D., F.J. Wooding, and E.H. Whiting (1978) Effects of off-road vehicle traffic on soils and vegetation in the Denali Highway region of Alaska. Journal of Soil and Water Conservation, 33: 20-27.

Swanson, F.J., T.K. Kratz, N. Caine, and R.G. Woodmansee (1988) Landform effects on ecosystem patterns and processes. Bioscience, 38: 92-98.

Tande, G.F. (1983) Vegetation. In Natural Resource Inventory of Elmendorf Air Force Base, Alaska: Part I (T.C. Rothe, S.H.Lanigan, P.A. Martin, and G.F. Tande). U.S. Fish and Wildlife Service, Anchorage, Alaska, Special Studies 14-85.

Thomson, J.W. (1984) American Arctic Lichens 1: The Macrolichens. New York: Columbia University Press, $504 \mathrm{p}$.

Uhling, P.W.C., and J.K. Jordan (1996) A spatial hierarchical framework for the co-management of ecosystems in Canada and the United States for the Upper Great Lakes Region. Environmental Monitoring and Assessment, 39: 59-73.

U.S. Army Alaska (USARAK) (1998) Integrated natural resources management plan, 1998-2003: Volume 2, Fort Richardson. Prepared by Natural Resources Branch, U.S. Army Alaska; Gene Stout and Associates; and Center for Ecological Management of Military Lands, Colorado State University. (http:// www.usarak.army.mil/conservation/fr_inrmp_old.htm) U.S. Army Alaska (USARAK) (2001) Fort Richardson's history. (http://www.usarak.army.mil/conservation/documents.htm).

Van Cleve, K. (1977) Recovery of disturbed tundra and taiga surfaces in Alaska. In Proceedings of the International Symposium on the Recovery of Damaged Ecosystems (J. Cairns, K.L. Dickson, and E.E. Herricks, Ed.), p. 422-455. Blacksburg, Virginia: Virginia Polytechnic Institute.

Van Cleve, K., C.T. Dyrness, L.A. Viereck, J. Fox, F.S. Chapin III, and W. Oechel (1983) Taiga ecosystems in Interior Alaska. Bioscience, 33: 39-44.

Van Cleve, K., F.S. Chapin III, C.T. Cyrness, and L.A. Viereck (1990) Element cycling in taiga forests: State-factor control. Bioscience, 41: 78-88.

Van Cleve, K., L.A. Viereck, and G.M. Marion (1993) Introduction and overview of a study dealing with the role of salt-affected soils in primary succession on the Tanana River floodplain, interior Alaska. Canadian Journal of Forest Research, 23: 879-888.

Viereck, L.A. (1970) Forest succession and soil devel- 
opment adjacent to the Chena River in interior Alaska. Arctic and Alpine Research, 2: 1-26.

Viereck, L.A (1973) Wildfire in the taiga of Alaska. Journal of Quaternary Research, 3: 465-495.

Viereck, L.A., and L.A. Schandelmeier (1980) Effects of fire in Alaska and adjacent Canada-A literature review. Bureau of Land Management, U.S. Department of Interior, Anchorage, Alaska, Technical Report 6, 76 p.

Viereck, L.A., C.T. Dyrness, K. Van Cleve, and M.J. Foote (1983) Vegetation, soils, and forest productivity in selected forest types in interior Alaska. Canadian Journal of Forestry, 13: 703-720.

Viereck, L.A., C.T. Dyrness, A.R. Batten, and K.J. Wenzlick (1992) The Alaska Vegetation Classification. Pacific Northwest Research Station, U.S. Forest Service, Portland, Oregon, General Technical Report PNWGTR-286, 278 p.

Viereck, L.A., C.T. Dyrness, and M.J. Foote (1993) Vegetation and soils of the floodplain ecosystems of the Tanana River, interior Alaska. Canadian Journal of Forest Research, 23: 889-898.

Vince, S.W., and A.A. Snow (1984) Plant zonation in an Alaska Salt Marsh. Journal of Ecology, 72: 651667.

Vitikainen, O. (1994) Taxonomic Revision of Peltigera (lichenized Ascomycotina) in Europe. Acta Botanica Fennica, 152: 1-96.

Vitousek, P.M. (1994) Factors controlling ecosystem structure and function. In Factors of Soil Formation: A Fiftieth Anniversary Retrospective (R. Amundsen, J. Harden, and M. Singer, Ed.), p. 87-97. Madison, Wisconsin: Soil Science Society of America, SSSA Special Publication 33.

Wahrhaftig, C. (1965) Physiographic Divisions of Alaska. U.S. Geological Survey, Washington, D.C., Professional Paper 482, 52 p.

Walker, D.A. (1983) A hierarchical tundra vegetation classification especially designed for mapping in northern Alaska. In Proceedings, Permafrost Fourth International Conference, University of Alaska, Fairbanks, Alaska, p. 1332-1337. Washington, D.C.: National Academy Press.

Walker, D.A (1999) An integrated vegetation mapping approach for northern Alaska (1:4 M scale). International Journal of Remote Sensing, 20: 2895-2920.

Walker, D.A., and K.R. Everett (1987) Road dust and its environmental impact on Alaska taiga and tundra. Arctic and Alpine Research, 19: 479-489.

Walker, D.A., and M.D. Walker (1991) History and pattern of disturbance in Alaskan arctic terrestrial ecosystems: A hierarchical approach to analyzing landscape change. Journal of Applied Ecology, 28: 244-276.

Walker, D.A., K.R. Everett, P.J. Webber, and J.
Brown (1980) Geobotanical atlas of the Prudhoe Bay region, Alaska. U.S. Army Cold Regions Research and Engineering Laboratory, Hanover, New Hampshire, Report 80-14, 69 p.

Walker, D.A., D. Cate, J. Brown, and C. Racine (1987) Disturbance and recovery of arctic Alaskan tundra terrain: A review of investigations. U.S. Army Cold Regions Research and Engineering Laboratory, Hanover, New Hampshire, CRREL Report 87-11.

Walker, D.A., S.A. Elias, N.A. Auerbach, and S.K. Short (1997) Alpine Biodiversity, Fort Richardson, Alaska. Unpublished report prepared for U.S. Army, Fort Richardson, Alaska, by Institute of Arctic and Alpine Research, University of Colorado, Boulder, Colorado, $65 \mathrm{p}$.

Walter, H. (1979) Vegetation of the Earth, and Ecological Systems of the Geobiosphere. New York: Springer-Verlag, $274 \mathrm{p}$.

Washburn, A.L. (1973) Periglacial Processes and Environments. London: Edward Arnold, 320 p.

Watt, A.S. (1947) Pattern and process in the plant community. Journal of Ecology, 35: 1-22.

Wiken, E.B. (1981) Ecological land classification: Analysis and methodologies. Lands Directorate, Environment Canada, Ottawa, Canada,. ELC Series No. 6. Wiken, E.B., and G. Ironside (1977) The development of ecological (biophysical) land classification in Canada. Landscape Planning, 4: 273-275.

Winkler, G.R., M.L. Miller, R.B. Hoekzema, and J.A. Dumoulin (1984) Guide to the bedrock geology of a traverse of the Chugach Mountains from Anchorage to Cape Resurrection. Alaska Geological Society, Anchorage, Alaska, $40 \mathrm{p}$.

Wittwer, D. (2000) Forest insect and disease conditions in Alaska-1999. U.S. Forest Service, Anchorage, Alaska, General Technical Report R10-TP-82, 55 p.

Yehle, L.A., and H.R. Schmoll (1987) Surficial geologic map of the Anchorage B-7 NW quadrangle, Alaska. U.S. Geological Survey, Washington, D.C., Open File Report 87-416.

Yehle, L.A., and H.R. Schmoll (1989) Surficial geologic map of the Anchorage B-7 SW quadrangle, Alaska. U.S. Geological Survey, Anchorage, Alaska, Open File Report 89-313.

Yehle, L.A., H.R. Schmoll, and E. Dobrovolny (1990) Surficial geologic map of the Anchorage B-8 SE and part of the Anchorage B-8 NE quadrangles, Alaska. U.S. Geological Survey, Anchorage, Alaska, Open File Report 90-238.

Yehle, L.A., H.R. Schmoll, and E. Dobrovolny (1991) Geologic map of the Anchorage B-8 SW quadrangle, Alaska. U.S. Geological Survey, Washington, D.C., Open File Report 92-350. 


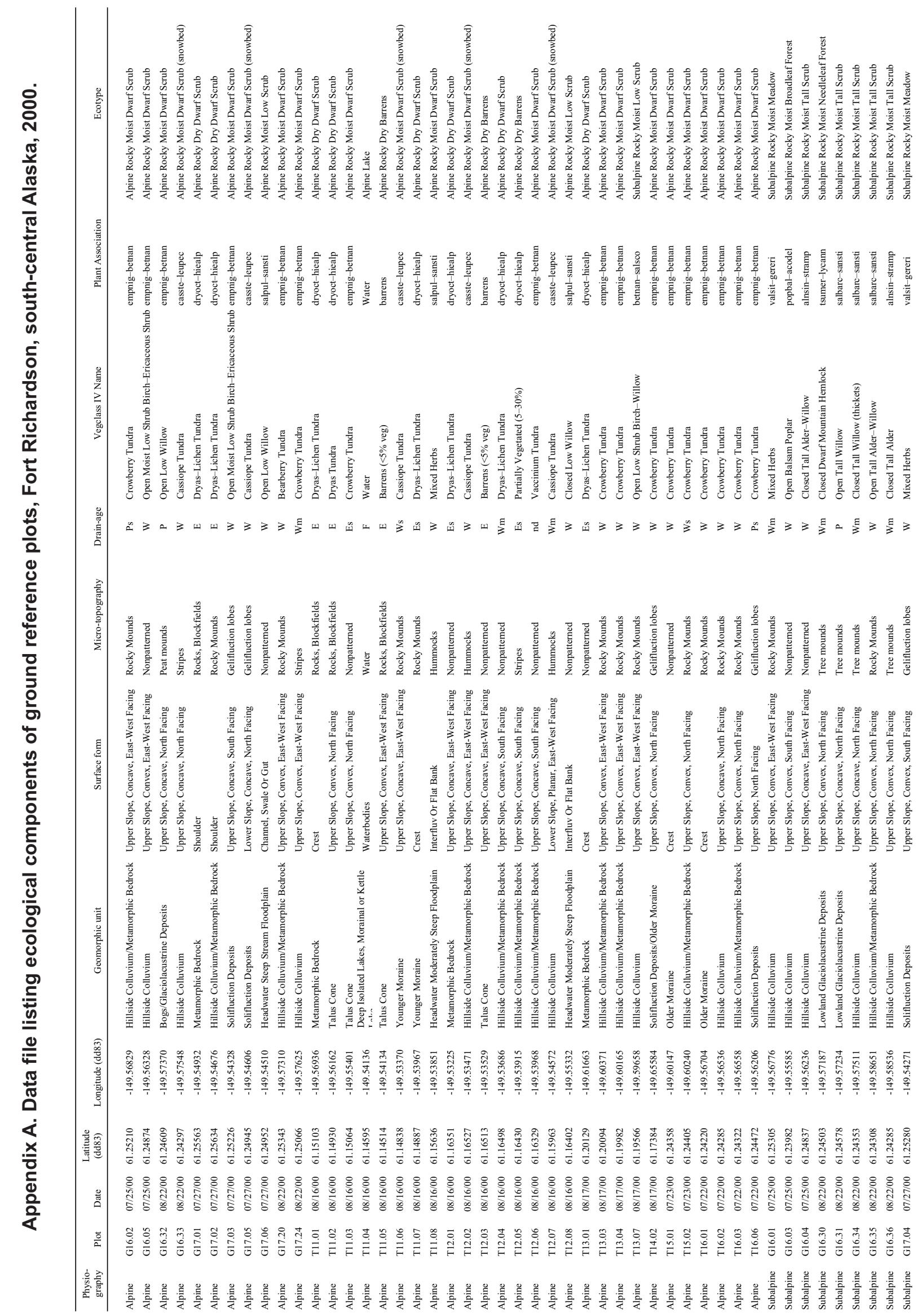









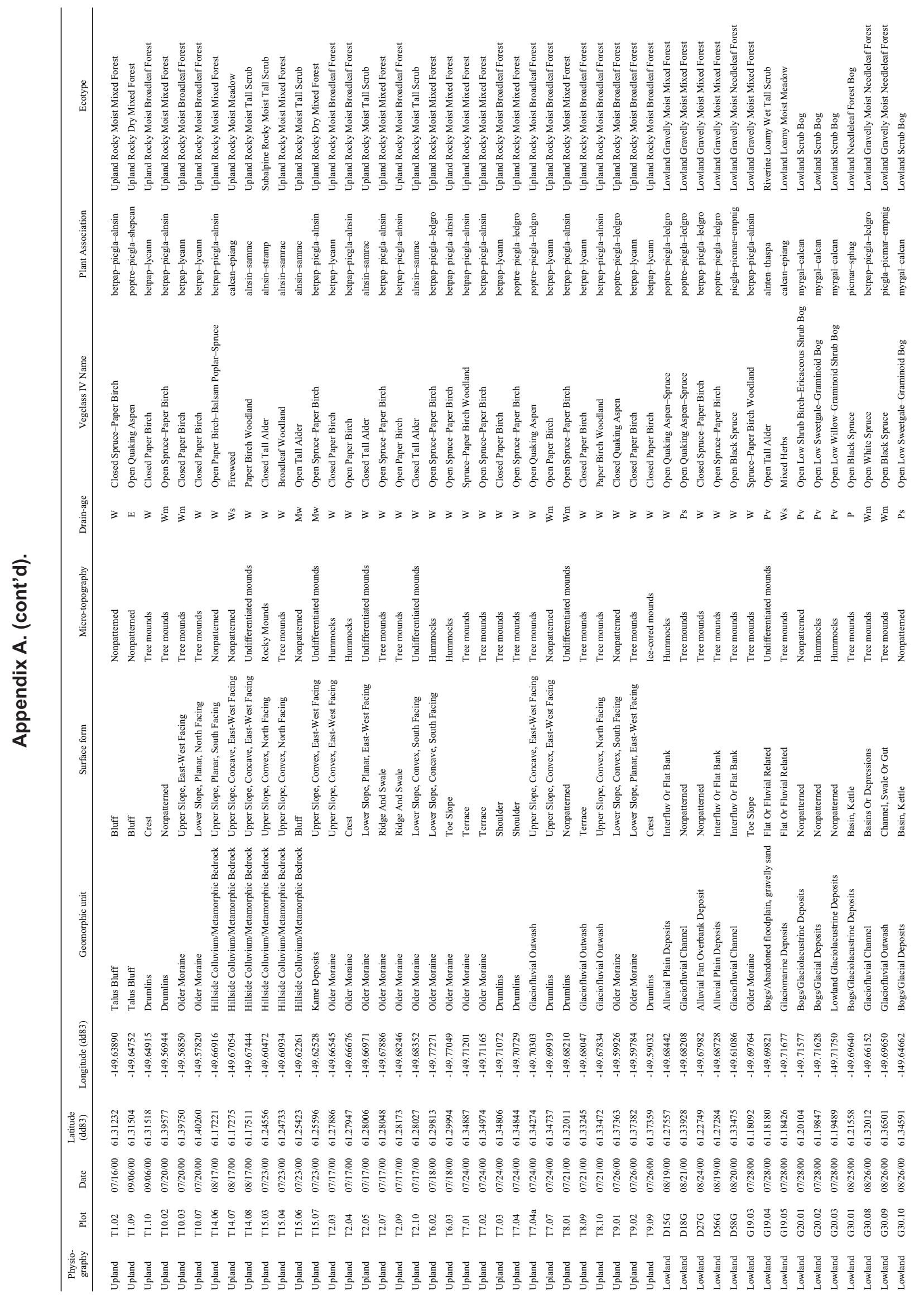




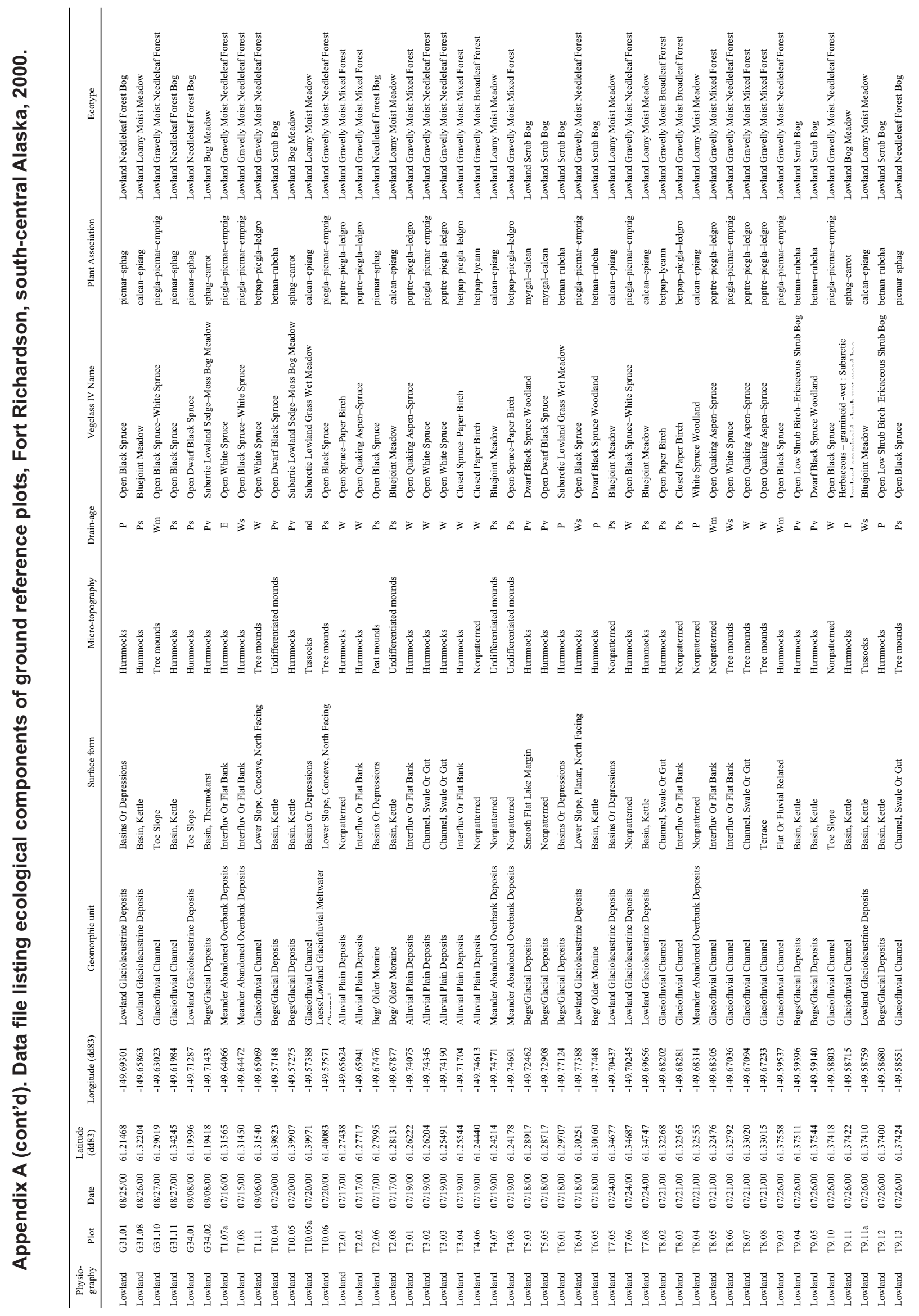




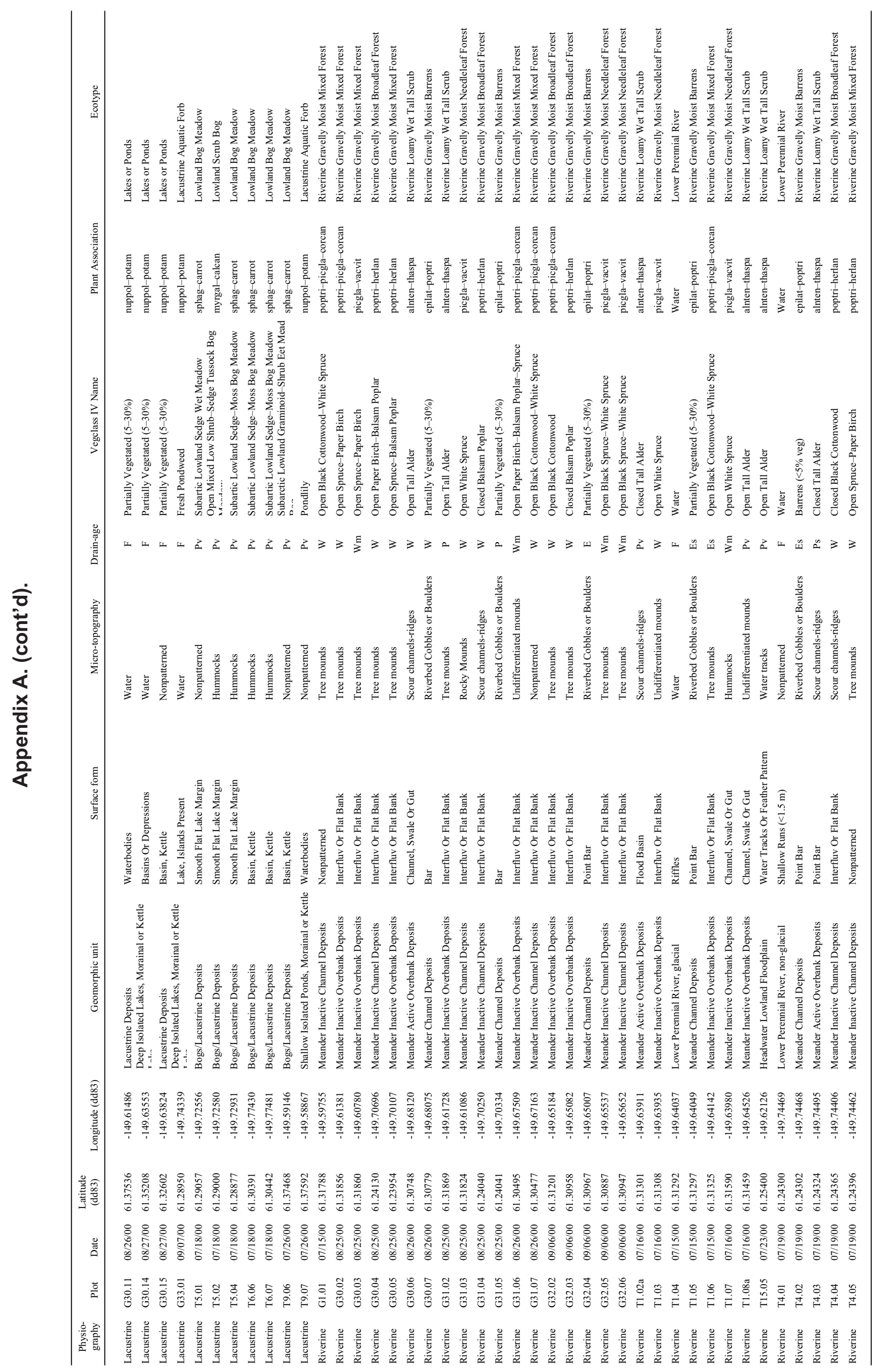









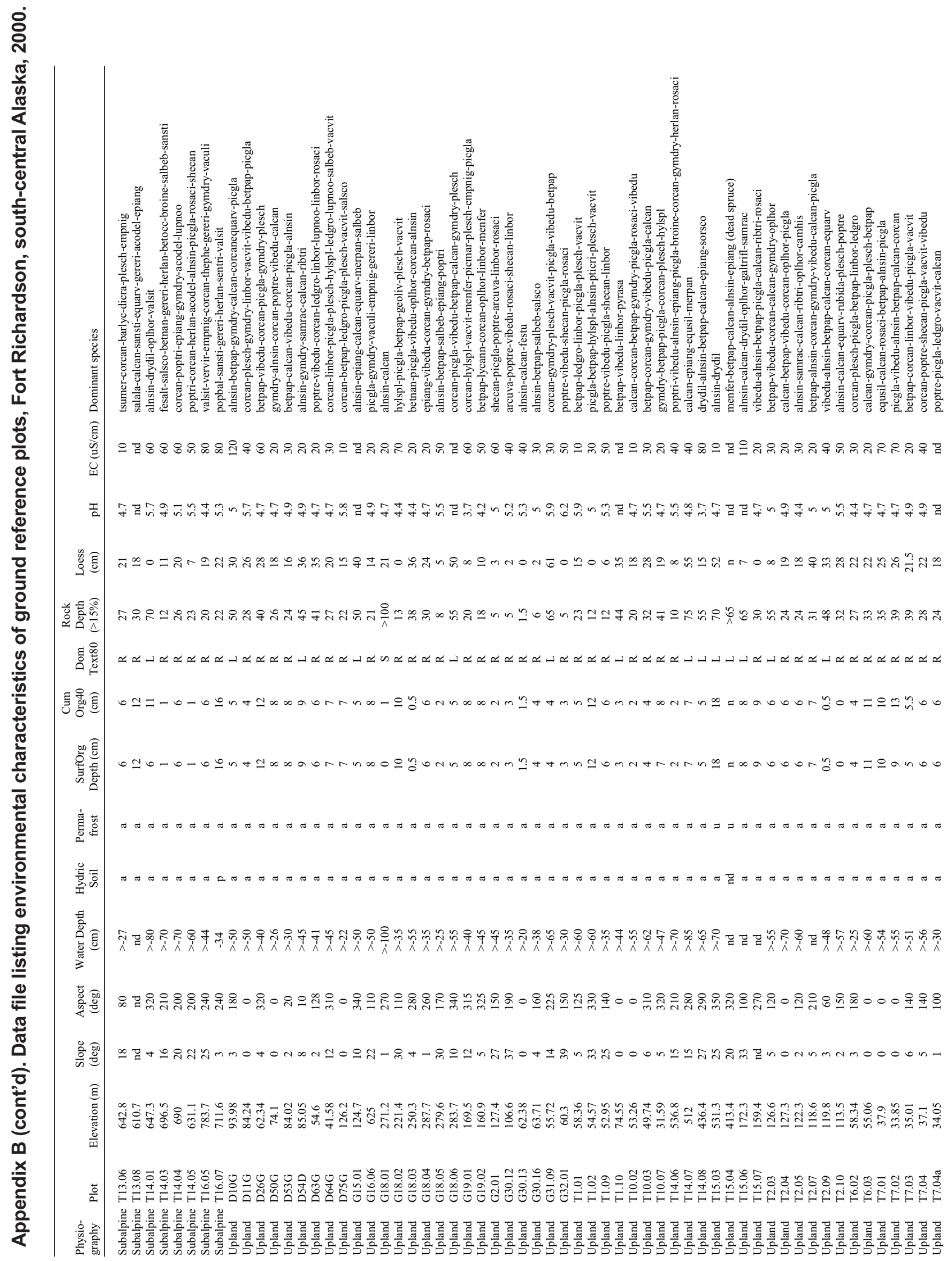




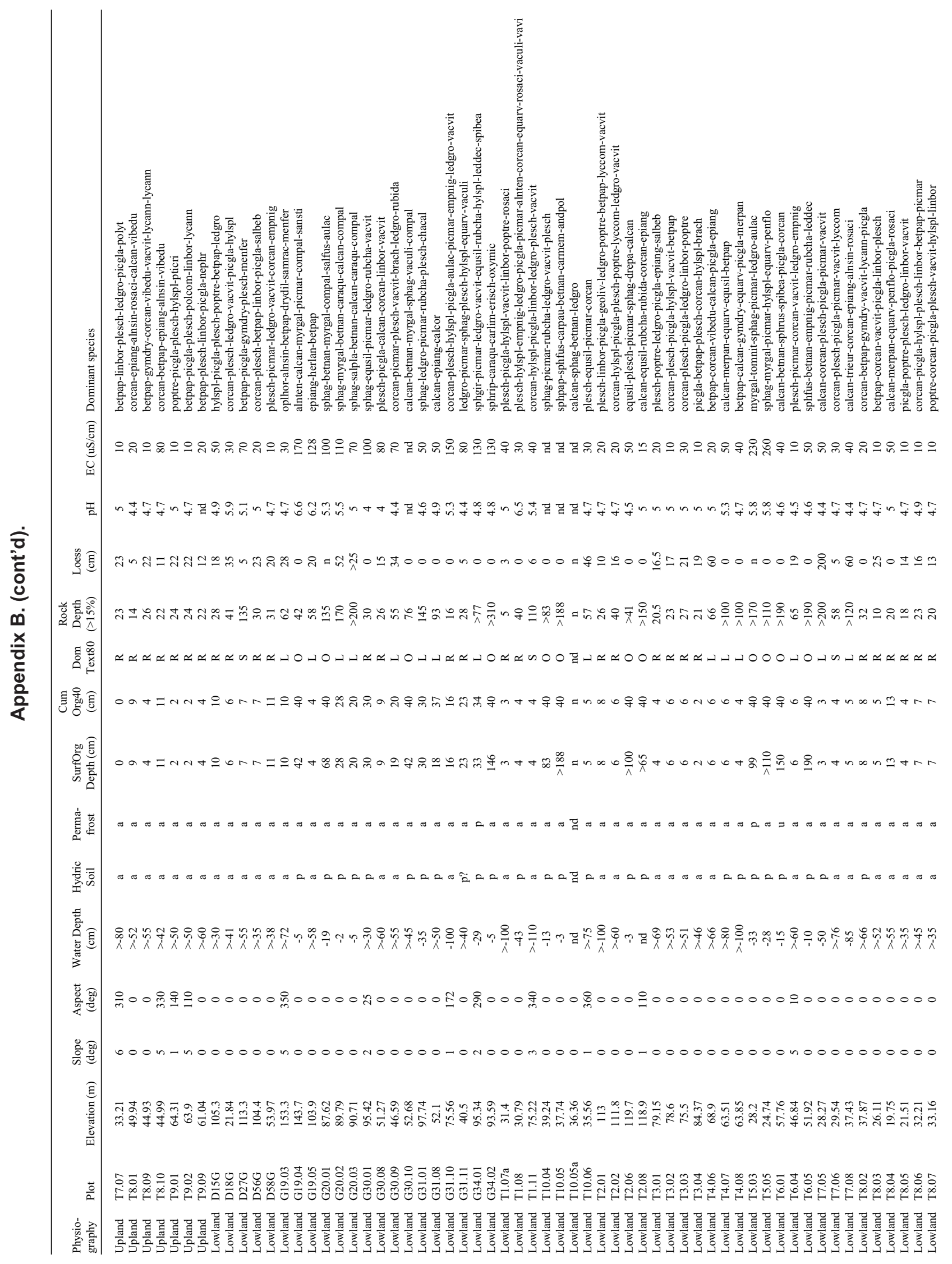




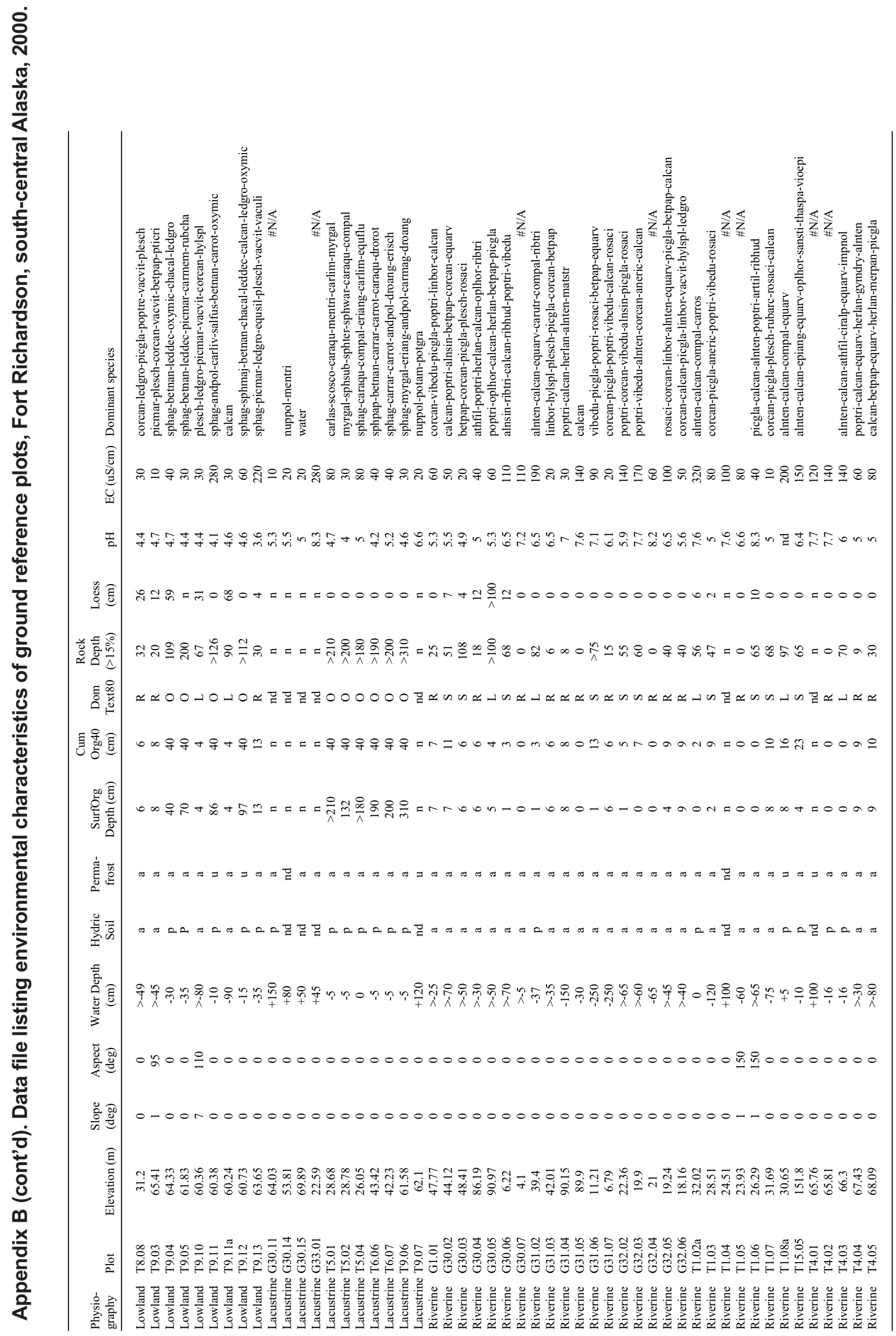


Appendix C. System for aggregating geomorphic units, surface forms, and vegetation classes into ecotype classes for Fort Richardson, south-central Alaska, 2001.

\begin{tabular}{|c|c|c|c|c|c|}
\hline Ecotype & ITU Code & Ecotype & ITU Code & Ecotype & ITU Code \\
\hline Alpine Rocky Dry Barrens & Cgy/Sle/Bpv/ & Alpine Rocky Moist Dwarf Scrub & Ctm/Sun/Sdec/ & Subalpine Rocky Moist Tall Scrub & $\mathrm{Ctm} / \mathrm{Sus} / \mathrm{Stca} /$ \\
\hline Alpine Rocky Dry Barrens & Cgy/Sin/Bpv/ & Alpine Rocky Moist Dwarf Scrub & $\mathrm{Ctm} /$ Sun/Sdee/ & Subalpine Rocky Moist Tall Scrub & $\mathrm{Ctm} /$ Sus/Stoa/ \\
\hline Alpine Rocky Dry Barrens & Cgy/Slv/Bbg/ & Alpine Rocky Moist Dwarf Scrub & $\mathrm{Ctm} / \mathrm{Sus} / \mathrm{Sdec} /$ & Subalpine Rocky Moist Broadleaf Forest & $\mathrm{Ctm} / \mathrm{SIn} /$ Fbop/ \\
\hline Alpine Rocky Dry Barrens & Cgy/Sus/Bbg/ & Alpine Rocky Moist Dwarf Scrub & $\mathrm{Ctm} /$ Sus/Sdee/ & Subalpine Rocky Moist Broadleaf Forest & $\mathrm{Ctm} / \mathrm{Sls} /$ Fbop/ \\
\hline Alpine Rocky Dry Barrens & Cgy/Sus/Bpv/ & Alpine Rocky Moist Dwarf Scrub & Ff/Sle/Sdee/ & Subalpine Rocky Moist Broadleaf Forest & Ctm/Sue/Fbop/ \\
\hline Alpine Rocky Dry Barrens & $\mathrm{Ctc} / \mathrm{Sle} / \mathrm{Bbg} /$ & Alpine Rocky Moist Dwarf Scrub & Fhm/SIc/Sdee/ & Subalpine Rocky Moist Broadleaf Forest & Ctm/Sun/Fbop/ \\
\hline Alpine Rocky Dry Barrens & $\mathrm{Ctc} / \mathrm{Sln} / \mathrm{Bbg} /$ & Alpine Rocky Moist Dwarf Scrub & Fhm/Sle/Sdee/ & Subalpine Rocky Moist Broadleaf Forest & Ctm/Sus/Fbob/ \\
\hline Alpine Rocky Dry Barrens & $\mathrm{Ctc} / \mathrm{SIn} / \mathrm{Bpv} /$ & Alpine Rocky Moist Dwarf Scrub & Gmy/B/Sdecl & Subalpine Rocky Moist Broadleaf Forest & Ctm/Sus/Fbop/ \\
\hline Alpine Rocky Dry Barrens & $\mathrm{Ctc} / \mathrm{Sue} / \mathrm{Bbg} /$ & Alpine Rocky Moist Dwarf Scrub & Gmy/B/Sdee/ & Subalpine Rocky Moist Broadleaf Forest & Gmo/Sls/Fbop/ \\
\hline Alpine Rocky Dry Barrens & $\mathrm{Ctc} / \mathrm{Sue} / \mathrm{Bpv} /$ & Alpine Rocky Moist Dwarf Scrub & Gmy/F/Sdee/ & Subalpine Rocky Moist Needleleaf Forest & $\mathrm{Ctm} / \mathrm{Sle} / \mathrm{Sfcmh} /$ \\
\hline Alpine Rocky Dry Barrens & $\mathrm{Ctc} / \mathrm{Sun} / \mathrm{Bbg} /$ & Alpine Rocky Moist Dwarf Scrub & Gmy/Sle/Sdecl & Subalpine Rocky Moist Needleleaf Forest & $\mathrm{Ctm} / \mathrm{Sln} / \mathrm{Sfcmh} /$ \\
\hline Alpine Rocky Dry Barrens & $\mathrm{Ctc} / \mathrm{Sun} / \mathrm{Bpv} /$ & Alpine Rocky Moist Dwarf Scrub & Gmy/Sle/Sdee/ & Subalpine Rocky Moist Needleleaf Forest & $\mathrm{Ctm} / \mathrm{Sls} / \mathrm{Sfcmh} /$ \\
\hline Alpine Rocky Dry Barrens & $\mathrm{Ctc} / \mathrm{Sus} / \mathrm{Bbg} /$ & Alpine Rocky Moist Dwarf Scrub & Gmy/SIn/Sdec/ & Subalpine Rocky Moist Needleleaf Forest & $\mathrm{Ctm} /$ Sue/Sfcmh/ \\
\hline Alpine Rocky Dry Barrens & $\mathrm{Ctc} / \mathrm{Sus} / \mathrm{Bpv} /$ & Alpine Rocky Moist Dwarf Scrub & Gmy/SIn/Sdee/ & Subalpine Rocky Moist Needleleaf Forest & Ctm/Sun/Fnows/ \\
\hline Alpine Rocky Dry Barrens & $\mathrm{Ctm} / \mathrm{C} / \mathrm{Bpv} /$ & Alpine Rocky Moist Dwarf Scrub & Gmy/SIs/Sdec/ & Subalpine Rocky Moist Needleleaf Forest & $\mathrm{Ctm} / \mathrm{Sun} / \mathrm{Sfcmh} /$ \\
\hline Alpine Rocky Dry Barrens & $\mathrm{Ctm} / \mathrm{Slc} / \mathrm{Bbg} /$ & Alpine Rocky Moist Dwarf Scrub & Gmy/SIs/Sdee/ & Subalpine Rocky Moist Needleleaf Forest & $\mathrm{Ctm} / \mathrm{Sus} / \mathrm{Sfcmh} /$ \\
\hline Alpine Rocky Dry Barrens & $\mathrm{Ctm} / \mathrm{Sle} / \mathrm{Bbg} /$ & Alpine Rocky Moist Dwarf Scrub & Gmy/Sue/Sdee/ & Subalpine Rocky Moist Needleleaf Forest & $\mathrm{Gmy} / \mathrm{Xm} / \mathrm{Sfcmh} /$ \\
\hline Alpine Rocky Dry Barrens & $\mathrm{Ctm} / \mathrm{Sle} / \mathrm{Bpv} /$ & Alpine Rocky Moist Dwarf Scrub & Gmy/Sun/Sdee/ & Upland Rocky Dry Barrens & $\mathrm{Ctb} / \mathrm{Sb} / \mathrm{Bbg} /$ \\
\hline Alpine Rocky Dry Barrens & $\mathrm{Ctm} / \mathrm{SIn} / \mathrm{Bbg} /$ & Alpine Rocky Moist Dwarf Scrub & Gmy/Sus/Sdee/ & Upland Rocky Dry Barrens & $\mathrm{Ctb} / \mathrm{Sb} / \mathrm{Bpv} /$ \\
\hline Alpine Rocky Dry Barrens & $\mathrm{Ctm} / \mathrm{SIn} / \mathrm{Bpv} /$ & Alpine Rocky Moist Dwarf Scrub & Gmy/Xm/Sdecl & Upland Rocky Dry Broadleaf Forest & $\mathrm{Ctb} / \mathrm{Sb} / \mathrm{Fbcal}$ \\
\hline Alpine Rocky Dry Barrens & $\mathrm{Ctm} / \mathrm{Sls} / \mathrm{Bbg} /$ & Alpine Rocky Moist Dwarf Scrub & Gmy/Xm/Sdee/ & Upland Rocky Dry Broadleaf Forest & $\mathrm{Ctb} / \mathrm{Sb} / \mathrm{Fboa} /$ \\
\hline Alpine Rocky Dry Barrens & $\mathrm{Ctm} / \mathrm{Suc} / \mathrm{Bbg} /$ & Alpine Rocky Moist Dwarf Scrub & $\mathrm{N} / \mathrm{Sus} / \mathrm{Sdee} /$ & Upland Rocky Dry Broadleaf Forest & $\mathrm{Ctb} / \mathrm{Sb} / \mathrm{Fboba} /$ \\
\hline Alpine Rocky Dry Barrens & $\mathrm{Ctm} / \mathrm{Sue} / \mathrm{Bbg} /$ & Alpine Rocky Moist Low Scrub & $\mathrm{Ctm} / \mathrm{SIc} / \mathrm{SIcw} /$ & Upland Rocky Dry Mixed Forest & Ctb/Sb/Fmoas/ \\
\hline Alpine Rocky Dry Barrens & $\mathrm{Ctm} / \mathrm{Sue} / \mathrm{Bpv} /$ & Alpine Rocky Moist Low Scrub & $\mathrm{Ctm} / \mathrm{Slc} / \mathrm{Slow} /$ & Upland Rocky Moist Tall Scrub & $\mathrm{Ctb} / \mathrm{Sb} / \mathrm{Stca} /$ \\
\hline Alpine Rocky Dry Barrens & $\mathrm{Ctm} / \mathrm{Sun} / \mathrm{Bpv} /$ & Alpine Rocky Moist Low Scrub & $\mathrm{Ctm} / \mathrm{Suc} / \mathrm{Slcw} /$ & Upland Rocky Moist Tall Scrub & $\mathrm{Ctb} / \mathrm{Sb} / \mathrm{Stcw} /$ \\
\hline Alpine Rocky Dry Barrens & $\mathrm{Ctm} / \mathrm{Sus} / \mathrm{Bbg} /$ & Alpine Rocky Moist Low Scrub & $\mathrm{Ctm} / \mathrm{Suc} / \mathrm{Slow} /$ & Upland Rocky Moist Tall Scrub & $\mathrm{Ctb} / \mathrm{Sb} / \mathrm{Stoa} /$ \\
\hline Alpine Rocky Dry Barrens & $\mathrm{Ctm} / \mathrm{Sus} / \mathrm{Bpv} /$ & Alpine Rocky Moist Low Scrub & $\mathrm{Fhm} / \mathrm{Slc} / \mathrm{Slcw} /$ & Upland Rocky Moist Tall Scrub & $\mathrm{Ctb} / \mathrm{Sb} / \mathrm{Stow} /$ \\
\hline Alpine Rocky Dry Barrens & $\mathrm{Gmy} / \mathrm{S} \ln / \mathrm{Bbg} /$ & Alpine Rocky Moist Low Scrub & Fhm/Slc/Slow/ & Upland Rocky Moist Tall Scrub & $\mathrm{Ctm} / \mathrm{Sls} / \mathrm{Stoa} /$ \\
\hline Alpine Rocky Dry Barrens & Gmy/SIn/Bpv/ & Alpine Rocky Moist Low Scrub & Fhm/Sle/Slow/ & Upland Rocky Moist Tall Scrub & $\mathrm{Ctm} / \mathrm{Suc} / \mathrm{Stca} /$ \\
\hline Alpine Rocky Dry Barrens & $\mathrm{N} / \mathrm{C} / \mathrm{Bbg} /$ & Alpine Rocky Moist Low Scrub & Fhm/Sln/Slowl & Upland Rocky Moist Tall Scrub & GFk/F/Stcal \\
\hline Alpine Rocky Dry Barrens & $\mathrm{N} / \mathrm{C} / \mathrm{Bpv} /$ & Alpine Rocky Moist Low Scrub & Fhm/Sun/Slow/ & Upland Rocky Moist Tall Scrub & GFk/Sue/Stoal \\
\hline Alpine Rocky Dry Barrens & N/Sue/Bbg/ & Alpine Lake & WIdim/W/W/ & Upland Rocky Moist Tall Scrub & GFk/Xm/Stcal \\
\hline Alpine Rocky Dry Barrens & N/Sue/Bpv/ & Subalpine Rocky Moist Meadow & $\mathrm{Cl} / \mathrm{Sue} / \mathrm{Hfmm} /$ & Upland Rocky Moist Tall Scrub & Gmo/F/Stoa/ \\
\hline Alpine Rocky Dry Barrens & $\mathrm{N} /$ Sun/Bpv/ & Subalpine Rocky Moist Meadow & $\mathrm{Ctb} / \mathrm{Sb} / \mathrm{Hfmm} /$ & Upland Rocky Moist Tall Scrub & Gmo/Sls/Stcal \\
\hline Alpine Rocky Dry Barrens & $\mathrm{N} / \mathrm{Sus} / \mathrm{Bpv} /$ & Subalpine Rocky Moist Meadow & $\mathrm{Ctm} / \mathrm{SIc} / \mathrm{Hfmm} /$ & Upland Rocky Moist Tall Scrub & Gmo/Sls/Stoa/ \\
\hline Alpine Rocky Dry Dwarf Scrub & Cgy/Sle/Sddl/ & Subalpine Rocky Moist Meadow & $\mathrm{Ctm} / \mathrm{Sle} / \mathrm{Hfmm} /$ & Upland Rocky Moist Tall Scrub & Gmo/Suc/Stcal \\
\hline Alpine Rocky Dry Dwarf Scrub & Cgy/Sus/Sddll/ & Subalpine Rocky Moist Meadow & $\mathrm{Ctm} / \mathrm{SIn} / \mathrm{Hfmm} /$ & Upland Rocky Moist Tall Scrub & Gmo/Suc/Stoal \\
\hline Alpine Rocky Dry Dwarf Scrub & $\mathrm{Cl} / \mathrm{Sun} / \mathrm{Sddl} /$ & Subalpine Rocky Moist Meadow & $\mathrm{Ctm} / \mathrm{Sls} / \mathrm{Hfmm} /$ & Upland Rocky Moist Tall Scrub & Gmo/Sue/Stcal \\
\hline Alpine Rocky Dry Dwarf Scrub & $\mathrm{Ctm} / \mathrm{C} / \mathrm{Sddl} /$ & Subalpine Rocky Moist Meadow & $\mathrm{Ctm} / \mathrm{Suc} / \mathrm{Hfmm} /$ & Upland Rocky Moist Tall Scrub & Gmo/Sun/Stcal \\
\hline Alpine Rocky Dry Dwarf Scrub & $\mathrm{Ctm} / \mathrm{Sle} / \mathrm{Sddl} /$ & Subalpine Rocky Moist Meadow & $\mathrm{Ctm} / \mathrm{Sue} / \mathrm{Hfmm} /$ & Upland Rocky Moist Tall Scrub & Gmo/Sus/Stca/ \\
\hline Alpine Rocky Dry Dwarf Scrub & $\mathrm{Ctm} / \mathrm{S} \ln / \mathrm{Sddl} /$ & Subalpine Rocky Moist Meadow & $\mathrm{Ctm} / \mathrm{Sun} / \mathrm{Hfmm} /$ & Upland Rocky Moist Tall Scrub & Gmo/Sus/Stowl \\
\hline Alpine Rocky Dry Dwarf Scrub & $\mathrm{Ctm} / \mathrm{S} \mid \mathrm{s} / \mathrm{Sddl} /$ & Subalpine Rocky Moist Meadow & $\mathrm{Ctm} / \mathrm{Sus} / \mathrm{Hfmm} /$ & Upland Rocky Moist Tall Scrub & $\mathrm{Gmo} / \mathrm{Xm} / \mathrm{Stca} /$ \\
\hline Alpine Rocky Dry Dwarf Scrub & $\mathrm{Ctm} / \mathrm{Sue} / \mathrm{Sddl} /$ & Subalpine Rocky Moist Low Scrub & $\mathrm{Cl} /$ Sun/Slobw/ & Upland Rocky Moist Broadleaf Forest & $\mathrm{Ctb} / \mathrm{Sb} / \mathrm{Fbcb} /$ \\
\hline Alpine Rocky Dry Dwarf Scrub & $\mathrm{Ctm} / \mathrm{Sun} / \mathrm{Sddl} /$ & Subalpine Rocky Moist Low Scrub & $\mathrm{Cl} /$ Sus/Slobw/ & Upland Rocky Moist Broadleaf Forest & $\mathrm{Ctb} / \mathrm{Sb} / \mathrm{Fbob} /$ \\
\hline Alpine Rocky Dry Dwarf Scrub & $\mathrm{Ctm} / \mathrm{Sus} / \mathrm{Sddl} /$ & Subalpine Rocky Moist Low Scrub & $\mathrm{Ctm} / \mathrm{SIc} / \mathrm{Slobw} /$ & Upland Rocky Moist Broadleaf Forest & $\mathrm{Ctm} / \mathrm{SIn} / \mathrm{Fbob} /$ \\
\hline Alpine Rocky Dry Dwarf Scrub & $\mathrm{Gmo} / \mathrm{C} / \mathrm{Sddl} /$ & Subalpine Rocky Moist Low Scrub & Ctm/Sle/Slobw/ & Upland Rocky Moist Broadleaf Forest & Ctm/Sus/Fbcal \\
\hline Alpine Rocky Dry Dwarf Scrub & Gmy/B/Sddl/ & Subalpine Rocky Moist Low Scrub & $\mathrm{Ctm} / \mathrm{SIn} / \mathrm{SIcw} /$ & Upland Rocky Moist Broadleaf Forest & Ctm/Sus/Fboa/ \\
\hline Alpine Rocky Dry Dwarf Scrub & $\mathrm{Gmy} / \mathrm{C} / \mathrm{Sddl} /$ & Subalpine Rocky Moist Low Scrub & $\mathrm{Ctm} / \mathrm{Sln} / \mathrm{Slobw} /$ & Upland Rocky Moist Broadleaf Forest & GFk/F/Fbcb/ \\
\hline Alpine Rocky Dry Dwarf Scrub & Gmy/SI/Sddl/ & Subalpine Rocky Moist Low Scrub & $\mathrm{Ctm} / \mathrm{Sls} / \mathrm{Slobw} /$ & Upland Rocky Moist Broadleaf Forest & GFk/F/Fbcba/ \\
\hline Alpine Rocky Dry Dwarf Scrub & Gmy/Sle/Sddl/ & Subalpine Rocky Moist Low Scrub & Ctm/Sue/Slobw/ & Upland Rocky Moist Broadleaf Forest & GFk/F/Fbob/ \\
\hline Alpine Rocky Dry Dwarf Scrub & Gmy/Sls/Sddl/ & Subalpine Rocky Moist Low Scrub & Ctm/Sun/Slobw/ & Upland Rocky Moist Broadleaf Forest & GFk/F/Fbobal \\
\hline Alpine Rocky Dry Dwarf Scrub & Gmy/Sue/Sddl/ & Subalpine Rocky Moist Low Scrub & Ctm/Sus/Slobw/ & Upland Rocky Moist Broadleaf Forest & GFk/Sle/Fbcb/ \\
\hline Alpine Rocky Dry Dwarf Scrub & $\mathrm{Gmy} / \mathrm{Xm} / \mathrm{Sddl} /$ & Subalpine Rocky Moist Low Scrub & Gmo/SIn/Slobw/ & Upland Rocky Moist Broadleaf Forest & GFk/Sle/Fbob/ \\
\hline Alpine Rocky Dry Dwarf Scrub & $\mathrm{N} / \mathrm{C} / \mathrm{Sddl} /$ & Subalnine Rocky Moist I ow Scrub & Gmo/Sue/Slobw & Upland Rocky Moist Broadleaf Forest & GFk/Sle/Fboba/ \\
\hline Alpine Rocky Dry Dwarf Scrub & N/Sue/Sddl/ & Subalpine Rocky Moist Low Scrub & & Upland Rocky Moist Broadleaf Forest & GFk/Sln/Fbcb/ \\
\hline Alpine Rocky Dry Dwarf Scrub & $\mathrm{N} /$ Sus/Sddl/ & Subalpine Rocky Moist Low Scrub & Gmy/SIn/Slobw/ & Upland Rocky Moist Broadleaf Forest & GFk/SIs/Fbcal \\
\hline Alpine Rocky Moist Dwarf Scrub & $\mathrm{Cl} /$ Sun/Sdee/ & Subalpine Rocky Moist Low Scrub & Gmy/Xm/Slobw/ & Upland Rocky Moist Broadleaf Forest & GFk/SIs/Fbcb/ \\
\hline Alpine Rocky Moist Dwarf Scrub & $\mathrm{Ctc} / \mathrm{SIn} / \mathrm{Sdee} /$ & Subalpine Rocky Moist Tall Scrub & Cl/Sun/Stcal & Upland Rocky Moist Broadleaf Forest & GFk/Sls/Fbcbal \\
\hline Alpine Rocky Moist Dwarf Scrub & $\mathrm{Ctm} / \mathrm{Slc} / \mathrm{Sdec} /$ & Subalpine Rocky Moist Tall Scrub & $\mathrm{Ctm} / \mathrm{C} / \mathrm{Stoal}$ & Upland Rocky Moist Broadleaf Forest & GFk/SIs/Fbob/ \\
\hline Alpine Rocky Moist Dwarf Scrub & $\mathrm{Ctm} / \mathrm{SIc} / \mathrm{Sdee} /$ & Subalpine Rocky Moist Tall Scrub & $\mathrm{Ctm} / \mathrm{SIc} / \mathrm{Stcw} /$ & Upland Rocky Moist Broadleaf Forest & GFk/SIs/Fboba/ \\
\hline Alpine Rocky Moist Dwarf Scrub & $\mathrm{Ctm} / \mathrm{Sle} / \mathrm{Sdec} /$ & Subalpine Rocky Moist Tall Scrub & $\mathrm{Ctm} / \mathrm{Sle} / \mathrm{Stca} /$ & Upland Rocky Moist Broadleaf Forest & GFk/Sue/Fbob/ \\
\hline Alpine Rocky Moist Dwarf Scrub & $\mathrm{Ctm} / \mathrm{Sle} / \mathrm{Sdee} /$ & Subalpine Rocky Moist Tall Scrub & $\mathrm{Ctm} / \mathrm{Sle} / \mathrm{Stoa} /$ & Upland Rocky Moist Broadleaf Forest & GFk/Sun/Fbcb/ \\
\hline Alpine Rocky Moist Dwarf Scrub & $\mathrm{Ctm} / \mathrm{S} \ln / \mathrm{Sdec} /$ & Subalpine Rocky Moist Tall Scrub & $\mathrm{Ctm} / \mathrm{SIn} / \mathrm{Stca} /$ & Upland Rocky Moist Broadleaf Forest & GFk/Sus/Fbcb/ \\
\hline Alpine Rocky Moist Dwarf Scrub & $\mathrm{Ctm} / \mathrm{S} \ln / \mathrm{Sdee} /$ & Subalpine Rocky Moist Tall Scrub & $\mathrm{Ctm} / \mathrm{SIn} / \mathrm{Stcw} /$ & Upland Rocky Moist Broadleaf Forest & GFk/Sus/Fbcba/ \\
\hline Alpine Rocky Moist Dwarf Scrub & $\mathrm{Ctm} / \mathrm{SIs} / \mathrm{Sdec} /$ & Subalpine Rocky Moist Tall Scrub & $\mathrm{Ctm} / \mathrm{SIn} / \mathrm{Stoa} /$ & Upland Rocky Moist Broadleaf Forest & GFk/Xm/Fbca/ \\
\hline Alpine Rocky Moist Dwarf Scrub & $\mathrm{Ctm} / \mathrm{Sls} / \mathrm{Sdee} /$ & Subalpine Rocky Moist Tall Scrub & $\mathrm{Ctm} / \mathrm{Sls} / \mathrm{Stca} /$ & Upland Rocky Moist Broadleaf Forest & GFk/Xm/Fbcb/ \\
\hline Alpine Rocky Moist Dwarf Scrub & $\mathrm{Ctm} / \mathrm{Suc} / \mathrm{Sdec} /$ & Subalpine Rocky Moist Tall Scrub & $\mathrm{Ctm} / \mathrm{Suc} / \mathrm{Stcw} /$ & Upland Rocky Moist Broadleaf Forest & GFk/Xm/Fbcbal \\
\hline Alpine Rocky Moist Dwarf Scrub & $\mathrm{Ctm} /$ Suc/Sdee/ & Subalpine Rocky Moist Tall Scrub & Ctm/Sue/Stca/ & Upland Rocky Moist Broadleaf Forest & GFk/Xm/Fboal \\
\hline Alpine Rocky Moist Dwarf Scrub & $\mathrm{Ctm} /$ Sue/Sdec/ & Subalpine Rocky Moist Tall Scrub & Ctm/Sue/Stoa/ & Upland Rocky Moist Broadleaf Forest & GFk/Xm/Fbob/ \\
\hline Alpine Rocky Moist Dwarf Scrub & $\mathrm{Ctm} /$ Sue/Sdee/ & Subalpine Rocky Moist Tall Scrub & $\mathrm{Ctm} / \mathrm{Sun} / \mathrm{Stca} /$ & Upland Rocky Moist Broadleaf Forest & GFk/Xm/Fbobal \\
\hline
\end{tabular}




\begin{tabular}{|c|c|c|c|c|c|}
\hline Ecotype & ITU Code & Ecotype & ITU Code & Ecotype & ITU Code \\
\hline Upland Rocky Moist Broadleaf Forest & Gmo/C/Fbcb/ & Upland Rocky Moist Mixed Forest & Gmo/Xm/Fmcas/ & Lowland Gravelly Moist Broadleaf Forest & MG/F/Fbob/ \\
\hline Upland Rocky Moist Broadleaf Forest & $\mathrm{Gmo} / \mathrm{F} / \mathrm{Fbcb} /$ & Upland Rocky Moist Mixed Forest & $\mathrm{Gmo} / \mathrm{Xm} / \mathrm{Fmcsb} /$ & Lowland Gravelly Moist Broadleaf Forest & $\mathrm{MG} / \mathrm{Sle} / \mathrm{Fbcb} /$ \\
\hline Upland Rocky Moist Broadleaf Forest & Gmo/F/Fbob/ & Upland Rocky Moist Mixed Forest & Gmo/Xm/Fmoas/ & Lowland Gravelly Moist Broadleaf Forest & MG/Sle/Fbob/ \\
\hline Upland Rocky Moist Broadleaf Forest & Gmo/Sb/Fbob/ & Upland Rocky Moist Mixed Forest & Gmo/Xm/Fmosb/ & Lowland Gravelly Moist Broadleaf Forest & $\mathrm{MG} / \mathrm{SIs} / \mathrm{Fbca} /$ \\
\hline Upland Rocky Moist Broadleaf Forest & $\mathrm{Gmo} / \mathrm{Slc} / \mathrm{Fbcb} /$ & Upland Rocky Moist Mixed Forest & Gmo/Xm/Fmosb & Lowland Gravelly Moist Broadleaf Forest & $\mathrm{MG} / \mathrm{Sls} / \mathrm{Fbcb} /$ \\
\hline Upland Rocky Moist Broadleaf Forest & Gmo/Sle/Fbcb/ & Upland Rocky Moist Needleleaf Forest & Ctb/Sb/Fnows/ & Lowland Gravelly Moist Mixed Forest & $\mathrm{Ff} / \mathrm{F} / \mathrm{Fmosb} /$ \\
\hline Upland Rocky Moist Broadleaf Forest & Gmo/Sle/Fbcba/ & Upland Rocky Moist Needleleaf Forest & $\mathrm{Ctm} / \mathrm{SIn} /$ Fnows/ & Lowland Gravelly Moist Mixed Forest & Ffo/F/Fmcsb/ \\
\hline Upland Rocky Moist Broadleaf Forest & Gmo/Sle/Fbob/ & Upland Rocky Moist Needleleaf Forest & Ctm/Sue/Fnows/ & Lowland Gravelly Moist Mixed Forest & $\mathrm{Ffo} / \mathrm{F} / \mathrm{Fmcsb}$ \\
\hline Upland Rocky Moist Broadleaf Forest & Gmo/Sle/Fboba/ & Upland Rocky Moist Needleleaf Forest & Ctm/Sus/Fnows/ & Lowland Gravelly Moist Mixed Forest & $\mathrm{Ffo} / \mathrm{F} / \mathrm{Fmosb} /$ \\
\hline Upland Rocky Moist Broadleaf Forest & $\mathrm{Gmo} / \mathrm{Sln} / \mathrm{Fbcb} /$ & Upland Rocky Moist Needleleaf Forest & GFk/F/Fnobw/ & Lowland Gravelly Moist Mixed Forest & Ffo/Slc/Fmosb/ \\
\hline Upland Rocky Moist Broadleaf Forest & $\mathrm{Gmo} / \mathrm{Sln} / \mathrm{Fbob} /$ & Upland Rocky Moist Needleleaf Forest & GFk/F/Fnows/ & Lowland Gravelly Moist Mixed Forest & $\mathrm{Ffo} / \mathrm{Sle} / \mathrm{Fmosb} /$ \\
\hline Upland Rocky Moist Broadleaf Forest & Gmo/Sls/Fbca/ & Upland Rocky Moist Needleleaf Forest & GFk/Sle/Fnobw/ & Lowland Gravelly Moist Mixed Forest & $\mathrm{Ffo} / \mathrm{SIn} / \mathrm{Fmcsb} /$ \\
\hline Upland Rocky Moist Broadleaf Forest & $\mathrm{Gmo} / \mathrm{Sls} / \mathrm{Fbcb} /$ & Upland Rocky Moist Needleleaf Forest & GFk/Sle/Fnows/ & Lowland Gravelly Moist Mixed Forest & $\mathrm{Ffo} / \mathrm{SIn} / \mathrm{Fmosb} /$ \\
\hline Upland Rocky Moist Broadleaf Forest & Gmo/Sls/Fboa/ & Upland Rocky Moist Needleleaf Forest & GFk/SIs/Fnobw/ & Lowland Gravelly Moist Mixed Forest & FGp/F/Fmcas/ \\
\hline Upland Rocky Moist Broadleaf Forest & Gmo/Sls/Fbob/ & Upland Rocky Moist Needleleaf Forest & GFk/Sue/Fnows/ & Lowland Gravelly Moist Mixed Forest & $\mathrm{FGp} / \mathrm{F} / \mathrm{Fmcsb} /$ \\
\hline Upland Rocky Moist Broadleaf Forest & Gmo/Sue/Fbcb/ & Upland Rocky Moist Needleleaf Forest & GFk/Xm/Fnows/ & Lowland Gravelly Moist Mixed Forest & FGp/F/Fmoas/ \\
\hline Upland Rocky Moist Broadleaf Forest & Gmo/Sue/Fboa/ & Upland Rocky Moist Needleleaf Forest & Gmo/F/Fnobw/ & Lowland Gravelly Moist Mixed Forest & $\mathrm{FGp} / \mathrm{F} / \mathrm{Fmosb} /$ \\
\hline Upland Rocky Moist Broadleaf Forest & Gmo/Sue/Fbob/ & Upland Rocky Moist Needleleaf Forest & Gmo/F/Fnows/ & Lowland Gravelly Moist Mixed Forest & FGp/Sle/Fmcsb/ \\
\hline Upland Rocky Moist Broadleaf Forest & Gmo/Sue/Fbobal & Upland Rocky Moist Needleleaf Forest & Gmo/Sle/Fnows/ & Lowland Gravelly Moist Mixed Forest & Fmob/F/Fmocws/ \\
\hline Upland Rocky Moist Broadleaf Forest & Gmo/Sun/Fbcb/ & Upland Rocky Moist Needleleaf Forest & Gmo/SIn/Fnows/ & Lowland Gravelly Moist Mixed Forest & Fmob/F/Fmosb/ \\
\hline Upland Rocky Moist Broadleaf Forest & Gmo/Sun/Fbob/ & Upland Rocky Moist Needleleaf Forest & Gmo/Sls/Fnobs/ & Lowland Gravelly Moist Mixed Forest & Fmrb/F/Fmosb/ \\
\hline Upland Rocky Moist Broadleaf Forest & Gmo/Sun/Fbobal & Upland Rocky Moist Needleleaf Forest & Gmo/Sls/Fnows/ & Lowland Gravelly Moist Mixed Forest & $\mathrm{Ft} / \mathrm{F} / \mathrm{Fmcsb} /$ \\
\hline Upland Rocky Moist Broadleaf Forest & Gmo/Sus/Fbcal & Upland Rocky Moist Needleleaf Forest & Gmo/Sue/Fnows/ & Lowland Gravelly Moist Mixed Forest & $\mathrm{Ft} / \mathrm{F} / \mathrm{Fmosb} /$ \\
\hline Upland Rocky Moist Broadleaf Forest & Gmo/Sus/Fbcb/ & Upland Rocky Moist Needleleaf Forest & Gmo/Sun/Fnows/ & Lowland Gravelly Moist Mixed Forest & $\mathrm{Ft} / \mathrm{Sls} / \mathrm{Fmcsb} /$ \\
\hline Upland Rocky Moist Broadleaf Forest & Gmo/Sus/Fbob/ & Upland Rocky Moist Needleleaf Forest & $\mathrm{Gmo} / \mathrm{Xm} /$ Fnobw/ & Lowland Gravelly Moist Mixed Forest & $\mathrm{GFc} / \mathrm{F} / \mathrm{Fmcsb} /$ \\
\hline Upland Rocky Moist Broadleaf Forest & Gmo/Sus/Fbobal & Upland Rocky Moist Needleleaf Forest & Gmo/Xm/Fnows/ & Lowland Gravelly Moist Mixed Forest & GFc/F/Fmoas/ \\
\hline Upland Rocky Moist Broadleaf Forest & Gmo/Xm/Fbca/ & Lowland Loamy Moist Meadow & $\mathrm{Fmob} / \mathrm{F} / \mathrm{Hgmb} /$ & Lowland Gravelly Moist Mixed Forest & GFc/F/Fmosb/ \\
\hline Upland Rocky Moist Broadleaf Forest & $\mathrm{Gmo} / \mathrm{Xm} / \mathrm{Fbcb} /$ & Lowland Loamy Moist Meadow & GFc/F/Hgmb/ & Lowland Gravelly Moist Mixed Forest & GFc/Slc/Fmosb/ \\
\hline Upland Rocky Moist Broadleaf Forest & Gmo/Xm/Fbcbal & Lowland Loamy Moist Meadow & GFc/SIc/Hgmb/ & Lowland Gravelly Moist Mixed Forest & GFc/Sle/Fmcsb/ \\
\hline Upland Rocky Moist Broadleaf Forest & Gmo/Xm/Fboal & Lowland Loamy Moist Meadow & GFk/B/Hgmb/ & Lowland Gravelly Moist Mixed Forest & GFc/Sle/Fmoas/ \\
\hline Upland Rocky Moist Broadleaf Forest & $\mathrm{Gmo} / \mathrm{Xm} / \mathrm{Fbob} /$ & Lowland Loamy Moist Meadow & GFk/F/Hgmb/ & Lowland Gravelly Moist Mixed Forest & GFc/Sle/Fmosb/ \\
\hline Upland Rocky Moist Broadleaf Forest & Gmo/Xm/Fbobal & Lowland Loamy Moist Meadow & GFo/F/Hgmb/ & Lowland Gravelly Moist Mixed Forest & $\mathrm{GFc} / \mathrm{SIn} / \mathrm{Fmcsb} /$ \\
\hline Upland Rocky Moist Mixed Forest & $\mathrm{Ctb} / \mathrm{Sb} / \mathrm{Fmcsb} /$ & Lowland Loamy Moist Meadow & $\mathrm{GL} / \mathrm{B} / \mathrm{Hgmb} /$ & Lowland Gravelly Moist Mixed Forest & GFc/SIn/Fmosb/ \\
\hline Upland Rocky Moist Mixed Forest & $\mathrm{Ctb} / \mathrm{Sb} / F m o s b /$ & Lowland Loamy Moist Meadow & $\mathrm{MG} / \mathrm{F} / \mathrm{Hgmb} /$ & Lowland Gravelly Moist Mixed Forest & GFc/Sls/Fmoas/ \\
\hline Upland Rocky Moist Mixed Forest & $\mathrm{Ctm} / \mathrm{SIn} / \mathrm{Fmcsb} /$ & Lowland Gravelly Moist Tall Scrub & Ffo/F/Stcal & Lowland Gravelly Moist Mixed Forest & GFc/Sls/Fmosb/ \\
\hline Upland Rocky Moist Mixed Forest & $\mathrm{Ctm} / \mathrm{Sln} / \mathrm{Fmosb} /$ & Lowland Gravelly Moist Tall Scrub & Ffo/Sls/Stcal & Lowland Gravelly Moist Mixed Forest & GFc/Xm/Fmoas/ \\
\hline Upland Rocky Moist Mixed Forest & Ctm/Sue/Fmosb/ & Lowland Gravelly Moist Tall Scrub & FGp/F/Stcal & Lowland Gravelly Moist Mixed Forest & $\mathrm{GFc} / \mathrm{Xm} / \mathrm{Fmosb} /$ \\
\hline Upland Rocky Moist Mixed Forest & $\mathrm{Ctm} / \mathrm{Sun} / \mathrm{Fmosb} /$ & Lowland Gravelly Moist Tall Scrub & FGp/F/Stoa/ & Lowland Gravelly Moist Mixed Forest & GFk/B/Fmosb/ \\
\hline Upland Rocky Moist Mixed Forest & Ctm/Sus/Fmcas/ & Lowland Gravelly Moist Tall Scrub & GFC/F/Stcal & Lowland Gravelly Moist Mixed Forest & GFo/F/Fmosb/ \\
\hline Upland Rocky Moist Mixed Forest & Ctm/Sus/Fmoas/ & Lowland Gravelly Moist Tall Scrub & GFC/F/Stoal & Lowland Gravelly Moist Mixed Forest & GFo/Sls/Fmosb/ \\
\hline Upland Rocky Moist Mixed Forest & Ctm/Sus/Fmosb/ & Lowland Gravelly Moist Broadleaf Forest & $\mathrm{Ff} / \mathrm{F} / \mathrm{Fbcb} /$ & Lowland Gravelly Moist Mixed Forest & $\mathrm{MG} / \mathrm{F} / \mathrm{Fmosb} /$ \\
\hline Upland Rocky Moist Mixed Forest & GFk/F/Fmcsb/ & Lowland Gravelly Moist Broadleaf Forest & $\mathrm{Ff} / \mathrm{F} / \mathrm{Fbob} /$ & Lowland Gravelly Moist Mixed Forest & $\mathrm{MG} / \mathrm{SI} / \mathrm{Fmos} /$ \\
\hline Upland Rocky Moist Mixed Forest & GFk/F/Fmoas/ & Lowland Gravelly Moist Broadleaf Forest & $\mathrm{Ffo} / \mathrm{F} / \mathrm{Fbcb} /$ & Lowland Gravelly Moist Mixed Forest & MG/Sle/Fmcsb/ \\
\hline Upland Rocky Moist Mixed Forest & GFk/F/Fmosb/ & Lowland Gravelly Moist Broadleaf Forest & $\mathrm{Ffo} / \mathrm{F} / \mathrm{Fbob} /$ & Lowland Gravelly Moist Mixed Forest & MG/Sle/Fmosb/ \\
\hline Upland Rocky Moist Mixed Forest & GFk/Sle/Fmcsb/ & Lowland Gravelly Moist Broadleaf Forest & Ffo/Sls/Fbob/ & Lowland Gravelly Moist Mixed Forest & MG/Sls/Fmosb/ \\
\hline Upland Rocky Moist Mixed Forest & GFk/Sle/Fmosb/ & Lowland Gravelly Moist Broadleaf Forest & $\mathrm{FGp} / \mathrm{F} / \mathrm{Fbca} /$ & Lowland Gravelly Moist Mixed Forest & $\mathrm{MG} / \mathrm{Xm} / \mathrm{Fmosb} /$ \\
\hline Upland Rocky Moist Mixed Forest & GFk/SIn/Fmosb/ & Lowland Gravelly Moist Broadleaf Forest & $\mathrm{FGp} / \mathrm{F} / \mathrm{Fbcb} /$ & Lowland Gravelly Moist Needleleaf Forest & Ffo/F/Fnows/ \\
\hline Upland Rocky Moist Mixed Forest & GFk/Sls/Fmcas/ & Lowland Gravelly Moist Broadleaf Forest & $\mathrm{FGp} / \mathrm{F} / \mathrm{Fbcba} /$ & Lowland Gravelly Moist Needleleaf Forest & FGp/F/Fnobw/ \\
\hline Upland Rocky Moist Mixed Forest & GFk/Sls/Fmcsb/ & Lowland Gravelly Moist Broadleaf Forest & FGp/F/Fboal & Lowland Gravelly Moist Needleleaf Forest & FGp/F/Fnows/ \\
\hline Upland Rocky Moist Mixed Forest & GFk/Sls/Fmosb/ & Lowland Gravelly Moist Broadleaf Forest & FGp/F/Fbob/ & Lowland Gravelly Moist Needleleaf Forest & Fmob/F/Fnows/ \\
\hline Upland Rocky Moist Mixed Forest & GFk/Sue/Fmosb/ & Lowland Gravelly Moist Broadleaf Forest & FGp/F/Fbobal & Lowland Gravelly Moist Needleleaf Forest & Fmrb/F/Fnobw/ \\
\hline Upland Rocky Moist Mixed Forest & GFk/Sun/Fmoas/ & Lowland Gravelly Moist Broadleaf Forest & $\mathrm{FGp} / \mathrm{F} / \mathrm{Fboc} /$ & Lowland Gravelly Moist Needleleaf Forest & GFc/B/Fnobw/ \\
\hline Upland Rocky Moist Mixed Forest & GFk/Sun/Fmosb/ & Lowland Gravelly Moist Broadleaf Forest & Fmob/F/Fbob/ & Lowland Gravelly Moist Needleleaf Forest & GFc/F/Fnobs/ \\
\hline Upland Rocky Moist Mixed Forest & GFk/Sus/Fmcsb/ & Lowland Gravelly Moist Broadleaf Forest & Fmrb/F/Fbob/ & Lowland Gravelly Moist Needleleaf Forest & GFc/F/Fnobw/ \\
\hline Upland Rocky Moist Mixed Forest & GFk/Xm/Fmcas/ & Lowland Gravelly Moist Broadleaf Forest & $\mathrm{Ft} / \mathrm{F} / \mathrm{Fbcb} /$ & Lowland Gravelly Moist Needleleaf Forest & GFc/F/Fnows/ \\
\hline Upland Rocky Moist Mixed Forest & GFk/Xm/Fmcsb/ & Lowland Gravelly Moist Broadleaf Forest & $\mathrm{Ft} / \mathrm{Sls} / \mathrm{Fbcb} /$ & Lowland Gravelly Moist Needleleaf Forest & GFc/SIc/Fnobs/ \\
\hline Upland Rocky Moist Mixed Forest & GFk/Xm/Fmoas/ & Lowland Gravelly Moist Broadleaf Forest & Ft/Sus/Fbcb/ & Lowland Gravelly Moist Needleleaf Forest & GFc/Slc/Fnobw/ \\
\hline Upland Rocky Moist Mixed Forest & GFk/Xm/Fmosb/ & Lowland Gravelly Moist Broadleaf Forest & Ft/Sus/Fbob/ & Lowland Gravelly Moist Needleleaf Forest & GFc/Sle/Fnobw/ \\
\hline Upland Rocky Moist Mixed Forest & Gmo/C/Fmcsb/ & Lowland Gravelly Moist Broadleaf Forest & GFc/F/Fbcb/ & Lowland Gravelly Moist Needleleaf Forest & GFc/SIn/Fnobw/ \\
\hline Upland Rocky Moist Mixed Forest & Gmo/C/Fmosb/ & Lowland Gravelly Moist Broadleaf Forest & GFc/F/Fbob/ & Lowland Gravelly Moist Needleleaf Forest & GFc/Sls/Fnobw/ \\
\hline Upland Rocky Moist Mixed Forest & Gmo/F/Fmosb/ & Lowland Gravelly Moist Broadleaf Forest & GFc/F/Fbobal & Lowland Gravelly Moist Needleleaf Forest & GFc/Sls/Fnows/ \\
\hline Upland Rocky Moist Mixed Forest & Gmo/Slc/Fmosb/ & Lowland Gravelly Moist Broadleaf Forest & GFc/Sle/Fbcb/ & Lowland Gravelly Moist Needleleaf Forest & GFk/B/Fnobs/ \\
\hline Upland Rocky Moist Mixed Forest & Gmo/Sle/Fmcsb/ & Lowland Gravelly Moist Broadleaf Forest & GFc/Sle/Fbcbal & Lowland Gravelly Moist Needleleaf Forest & GFk/B/Fnobw/ \\
\hline Upland Rocky Moist Mixed Forest & Gmo/Sle/Fmosb/ & Lowland Gravelly Moist Broadleaf Forest & GFc/Sle/Fbob/ & Lowland Gravelly Moist Needleleaf Forest & GFk/B/Fnows/ \\
\hline Upland Rocky Moist Mixed Forest & Gmo/Sln/Fmosb/ & Lowland Gravelly Moist Broadleaf Forest & $\mathrm{GFc} / \mathrm{Sln} / \mathrm{Fbcb} /$ & Lowland Gravelly Moist Needleleaf Forest & GFo/F/Fnobw/ \\
\hline Upland Rocky Moist Mixed Forest & Gmo/Sls/Fmoas/ & Lowland Gravelly Moist Broadleaf Forest & $\mathrm{GFc} / \mathrm{Sln} / \mathrm{Fbob} /$ & Lowland Gravelly Moist Needleleaf Forest & GFo/Sls/Fnobw/ \\
\hline Upland Rocky Moist Mixed Forest & Gmo/Sls/Fmosb/ & Lowland Gravelly Moist Broadleaf Forest & $\mathrm{GFc} / \mathrm{Sls} / \mathrm{Fbcb} /$ & Lowland Gravelly Moist Needleleaf Forest & GL/B/Fnobs/ \\
\hline Upland Rocky Moist Mixed Forest & Gmo/Sue/Fmosb/ & Lowland Gravelly Moist Broadleaf Forest & $\mathrm{GFc} / \mathrm{Xm} / \mathrm{Fbcb} /$ & Lowland Gravelly Moist Needleleaf Forest & MG/F/Fnobs/ \\
\hline Upland Rocky Moist Mixed Forest & Gmo/Sun/Fmosb/ & Lowland Gravelly Moist Broadleaf Forest & GFc/Xm/Fboa/ & Lowland Gravelly Moist Needleleaf Forest & MG/SIc/Fnobs/ \\
\hline Upland Rocky Moist Mixed Forest & Gmo/Sus/Fmcsb/ & Lowland Gravelly Moist Broadleaf Forest & GFo/F/Fbobal & Lowland Gravelly Moist Needleleaf Forest & MG/Xm/Fnobs/ \\
\hline Upland Rocky Moist Mixed Forest & Gmo/Sus/Fmoas/ & Lowland Gravelly Moist Broadleaf Forest & GFo/Sls/Fbcb/ & Lowland Bog Meadow & $\mathrm{Ob} / \mathrm{B} / \mathrm{Hgwsmb} /$ \\
\hline Upland Rocky Moist Mixed Forest & Gmo/Sus/Fmosb/ & Lowland Gravelly Moist Broadleaf Forest & $\mathrm{MG} / \mathrm{F} / \mathrm{Fbcb} /$ & Lowland Bog Meadow & $\mathrm{Ob} / \mathrm{Bt} / \mathrm{Hgwsmb} /$ \\
\hline
\end{tabular}




\begin{tabular}{|c|c|c|c|c|c|}
\hline Ecotype & ITU Code & Ecotype & ITU Code & Ecotype & ITU Code \\
\hline Lowland Bog Meadow & $\mathrm{Ob} / \mathrm{F} / \mathrm{Hgwsmb} /$ & Coastal Sandy Moist Meadow & $\mathrm{Mta} / \mathrm{F} / \mathrm{Hgdl} /$ & Human Modified Meadow & $\mathrm{GFc} / \mathrm{F} / \mathrm{Hgmd} / \mathrm{Hcn}$ \\
\hline Lowland Scrub Bog & Ob/B/Sfobs/ & Coastal Sandy Moist Meadow & Mta/F//Hgdl/ & Human Modified Meadow & GFc/Sle/Hgmd/Hch \\
\hline Lowland Scrub Bog & $\mathrm{Ob} / \mathrm{B} / \mathrm{Slobb} /$ & Coastal Loamy Wet Low Scrub & Mti/F/Slomg/ & Human Modified Meadow & GFc/Sls/Hgmd/Hch \\
\hline Lowland Scrub Bog & Ob/B/Slocg/ & Coastal Lake and Marsh & $\mathrm{Mta} / \mathrm{Ff} / \mathrm{Hgwhsm} /$ & Human Modified Meadow & $\mathrm{GFc} / \mathrm{Sls} / \mathrm{Hgmd} / \mathrm{Hcn}$ \\
\hline Lowland Scrub Bog & Ob/F/Sfobs/ & Coastal Lake and Marsh & Welt/W/Wb/ & Human Modified Meadow & $\mathrm{GFk} / \mathrm{F} / \mathrm{Hgmd} / \mathrm{Hch}$ \\
\hline Lowland Scrub Bog & $\mathrm{Ob} / \mathrm{F} / \mathrm{Slobb} /$ & Nearshore Water & $\mathrm{Wmn} / \mathrm{W} / \mathrm{Wm} /$ & Human Modified Meadow & $\mathrm{GFk} / \mathrm{F} / \mathrm{Hgmd} / \mathrm{Hcn}$ \\
\hline Lowland Scrub Bog & Ob/F/Slocg/ & Human Modified Barrens & $\mathrm{Ctb} / \mathrm{Sb} / \mathrm{Bpv} / \mathrm{Hcn}$ & Human Modified Meadow & GFk/F/Hgmd/Hcs \\
\hline Lowland Needleleaf Forest Bog & Ob/B/Fnobs/ & Human Modified Barrens & $\mathrm{Ctm} / \mathrm{C} / \mathrm{Bbg} / \mathrm{Hcs}$ & Human Modified Meadow & GFk/Sle/Hgmd/Hcn \\
\hline Lowland Needleleaf Forest Bog & Ob/F/Fnobs/ & Human Modified Barrens & $\mathrm{Ctm} / \mathrm{SIn} / \mathrm{Bbg} / \mathrm{Ht}$ & Human Modified Meadow & GFk/SIn/Hgmd/Hch \\
\hline Lowland Needleleaf Forest Bog & Ob/Slc/Fnobs/ & Human Modified Barrens & $\mathrm{Ctm} / \mathrm{Sls} / \mathrm{Bpv} / \mathrm{Hcs}$ & Human Modified Meadow & GFk/SIs/Hgmd/Hcn \\
\hline Lowland Lake and Aquatic Forb & WIdim/W/Hafl/ & Human Modified Barrens & $\mathrm{Ctm} / \mathrm{Sue} / \mathrm{Bpv} / \mathrm{Hcs}$ & Human Modified Meadow & $\mathrm{Gmo} / \mathrm{F} / \mathrm{Hgmd} / \mathrm{Hch}$ \\
\hline Lowland Lake and Aquatic Forb & WIdim/W/WI & Human Modified Barrens & $\mathrm{Ctm} / \mathrm{Sus} / \mathrm{Bpv} / \mathrm{Hcs}$ & Human Modified Meadow & $\mathrm{Gmo} / \mathrm{F} / \mathrm{Hgmd} / \mathrm{Hcn}$ \\
\hline Lowland Lake and Aquatic Forb & Wlsim/W/Hafl/ & Human Modified Barrens & $\mathrm{Ffo} / \mathrm{F} / \mathrm{Bbg} / \mathrm{Ht}$ & Human Modified Meadow & $\mathrm{Gmo} / \mathrm{F} / \mathrm{Hgmd} / \mathrm{Hcs}$ \\
\hline Lowland Lake and Aquatic Forb & Wlsim/W/W/ & Human Modified Barrens & $\mathrm{Ffo} / \mathrm{F} / \mathrm{Bpv} / \mathrm{Hcs}$ & Human Modified Meadow & $\mathrm{Gmo} / \mathrm{Sls} / \mathrm{Hgmd} / \mathrm{Hcn}$ \\
\hline Riverine Gravelly Moist Barrens & Fmra/F/Bbg/ & Human Modified Barrens & $\mathrm{Ffo} / \mathrm{Xm} / \mathrm{Bpv} / \mathrm{Hcs}$ & Human Modified Meadow & $\mathrm{Gmo} / \mathrm{Xm} / \mathrm{Hgmd} / \mathrm{Hcn}$ \\
\hline Riverine Gravelly Moist Barrens & Fmra/F/Bpv/ & Human Modified Barrens & $\mathrm{FGp} / \mathrm{F} / \mathrm{Bbg} / \mathrm{Hcs}$ & Human Modified Meadow & $\mathrm{Gmo} / \mathrm{Xm} / \mathrm{Hgmd} / \mathrm{Hcs}$ \\
\hline Riverine Gravelly Moist Barrens & Fmra/Fbp/Bbg/ & Human Modified Barrens & $\mathrm{FGp} / \mathrm{F} / \mathrm{Bbg} / \mathrm{Ht}$ & Human Modified Meadow & $\mathrm{He} / \mathrm{B} / \mathrm{Hgmd} / \mathrm{He}$ \\
\hline Riverine Gravelly Moist Barrens & Fmra/Fbp/Bpv/ & Human Modified Barrens & $\mathrm{FGp} / \mathrm{F} / \mathrm{Bpv} / \mathrm{Hcn}$ & Human Modified Meadow & $\mathrm{Hf} / \mathrm{Hm} / \mathrm{Hgmd} / \mathrm{He}$ \\
\hline Riverine Gravelly Moist Barrens & Fmra/Fi/Bpv/ & Human Modified Barrens & $\mathrm{FGp} / \mathrm{F} / \mathrm{Bpv} / \mathrm{Hcs}$ & Human Modified Meadow & $\mathrm{Hf} / \mathrm{Hm} / \mathrm{Hgmd} / \mathrm{Hf}$ \\
\hline Riverine Gravelly Moist Barrens & Fmri/Fc/Bbg/ & Human Modified Barrens & $\mathrm{FGp} / \mathrm{F} / \mathrm{Bpv} / \mathrm{Ht}$ & Human Modified Meadow & $\mathrm{MG} / \mathrm{F} / \mathrm{Hgmd} / \mathrm{Hcn}$ \\
\hline Riverine Loamy Wet Tall Scrub & Fhl/F/Slow/ & Human Modified Barrens & $\mathrm{Fmra} / \mathrm{F} / \mathrm{Bbg} / \mathrm{Hcn}$ & Human Modified Scrub & $\mathrm{Ctb} / \mathrm{Sb} / \mathrm{Slod} / \mathrm{Hcs}$ \\
\hline Riverine Loamy Wet Tall Scrub & Fmoa/F/Stca/ & Human Modified Barrens & Fmrb///Bpv/Hcs & Human Modified Scrub & $\mathrm{Ctb} / \mathrm{Sb} / \mathrm{Slow} / \mathrm{Hcn}$ \\
\hline Riverine Loamy Wet Tall Scrub & Fmoa/Fbp/Stca/ & Human Modified Barrens & $\mathrm{Ft} / \mathrm{F} / \mathrm{Bpv} / \mathrm{Hcs}$ & Human Modified Scrub & $\mathrm{Ctb} / \mathrm{Sb} / \mathrm{Stca} / \mathrm{Hcn}$ \\
\hline Riverine Loamy Wet Tall Scrub & Fmoi/F/Stca/ & Human Modified Barrens & $\mathrm{GFC} / \mathrm{F} / \mathrm{Bbg} / \mathrm{Hcs}$ & Human Modified Scrub & $\mathrm{Ctb} / \mathrm{Sb} / \mathrm{Stoa} / \mathrm{Hcn}$ \\
\hline Riverine Loamy Wet Tall Scrub & Fmra/F/Stca/ & Human Modified Barrens & $\mathrm{GF} / \mathrm{F} / \mathrm{Bpv} / \mathrm{Hcs}$ & Human Modified Scrub & $\mathrm{Ctb} / \mathrm{Sb} / \mathrm{Stod} / \mathrm{Hcn}$ \\
\hline Riverine Loamy Wet Tall Scrub & Fmra/F/Stoal & Human Modified Barrens & GFk/F/Bpv/Hcn & Human Modified Scrub & $\mathrm{Ctb} / \mathrm{Sb} / \mathrm{Stod} / \mathrm{Hcs}$ \\
\hline Riverine Loamy Wet Tall Scrub & $\mathrm{Fmri} / \mathrm{F} / \mathrm{Stca} /$ & Human Modified Barrens & GFk/F/Bpv/Hcs & Human Modified Scrub & $\mathrm{Ctm} / \mathrm{Sls} / \mathrm{Stca} / \mathrm{Hcs}$ \\
\hline Riverine Loamy Wet Tall Scrub & Fmri/Fc/Stoal & Human Modified Barrens & GFk/Sus/Bpv/Hcs & Human Modified Scrub & $\mathrm{Ctm} /$ Sue/Slobw/Hcn \\
\hline Riverine Gravelly Moist Tall Scrub & $\mathrm{Fhm} / \mathrm{Slc} / \mathrm{Stca} /$ & Human Modified Barrens & $\mathrm{GFk} / \mathrm{Xm} / \mathrm{Bbg} / \mathrm{Ht}$ & Human Modified Scrub & $\mathrm{Ctm} / \mathrm{Sus} / \mathrm{Slod} / \mathrm{Hcn}$ \\
\hline Riverine Gravelly Moist Tall Scrub & $\mathrm{Fhm} / \mathrm{Slc} / \mathrm{Stcw} /$ & Human Modified Barrens & $\mathrm{GFk} / \mathrm{Xm} / \mathrm{Bpv} / \mathrm{Hcs}$ & Human Modified Scrub & $\mathrm{Ctm} / \mathrm{Sus} / \mathrm{Slod} / \mathrm{Hcs}$ \\
\hline Riverine Gravelly Moist Tall Scrub & Fhm/Slc/Stow/ & Human Modified Barrens & $\mathrm{Gmo} / \mathrm{C} / \mathrm{Bbg} / \mathrm{Ht}$ & Human Modified Scrub & $\mathrm{Ctm} / \mathrm{Sus} / \mathrm{Stca} / \mathrm{Hcn}$ \\
\hline Riverine Gravelly Moist Tall Scrub & $\mathrm{Fhm} / \mathrm{Slc} / \mathrm{Stow}$ & Human Modified Barrens & Gmo/F/Bpv/Hcs & Human Modified Scrub & $\mathrm{Ctm} / \mathrm{Sus} / \mathrm{Stca} / \mathrm{Hcs}$ \\
\hline Riverine Gravelly Moist Tall Scrub & $\mathrm{Fhm} / \mathrm{Sln} / \mathrm{Stow} /$ & Human Modified Barrens & $\mathrm{Gmo} / \mathrm{Sle} / \mathrm{Bpv} / \mathrm{Hcn}$ & Human Modified Scrub & $\mathrm{Ctm} / \mathrm{Sus} / \mathrm{Stod} / \mathrm{Hcn}$ \\
\hline Riverine Gravelly Moist Tall Scrub & $\mathrm{Fhm} / \mathrm{St} / \mathrm{Stcwl}$ & Human Modified Barrens & $\mathrm{Gmo} / \mathrm{Xm} / \mathrm{Bbg} / \mathrm{Ht}$ & Human Modified Scrub & $\mathrm{Ctm} / \mathrm{Sus} / \mathrm{Stod} / \mathrm{Hcs}$ \\
\hline Riverine Gravelly Moist Tall Scrub & Fhm/Suc/Stcal & Human Modified Barrens & $\mathrm{Gmo} / \mathrm{Xm} / \mathrm{Bpv} / \mathrm{Hcs}$ & Human Modified Scrub & $\mathrm{Ffo} / \mathrm{F} / \mathrm{Slod} / \mathrm{Hcn}$ \\
\hline Riverine Gravelly Moist Tall Scrub & Fhm/Suc/Stcw/ & Human Modified Barrens & $\mathrm{Gmo} / \mathrm{Xm} / \mathrm{Bpv} / \mathrm{Ht}$ & Human Modified Scrub & Ffo/F/Slod/Hcs \\
\hline Riverine Gravelly Moist Tall Scrub & Fhm/Suc/Stowl & Human Modified Barrens & $\mathrm{He} / \mathrm{B} / \mathrm{Bpv} / \mathrm{He}$ & Human Modified Scrub & $\mathrm{Ffo} / \mathrm{F} / \mathrm{Slod} / \mathrm{Ht}$ \\
\hline Riverine Gravelly Moist Broadleaf Forest & Fhl/F/Fbob/ & Human Modified Barrens & $\mathrm{He} / \mathrm{F} / \mathrm{Bpv} / \mathrm{Hwd}$ & Human Modified Scrub & $\mathrm{Ffo} / \mathrm{F} / \mathrm{Stca} / \mathrm{Hen}$ \\
\hline Riverine Gravelly Moist Broadleaf Forest & Fhm/Fi/Fbocl & Human Modified Barrens & $\mathrm{He} / \mathrm{Hm} / \mathrm{Bbg} / \mathrm{He}$ & Human Modified Scrub & Ffo/F/Stcw/Hch \\
\hline Riverine Gravelly Moist Broadleaf Forest & Fmri/F/Fbob/ & Human Modified Barrens & $\mathrm{He} / \mathrm{Hm} / \mathrm{Bpv} / \mathrm{He}$ & Human Modified Scrub & Ffo/F/Stoa/Hch \\
\hline Riverine Gravelly Moist Broadleaf Forest & Fmri/F/Fbocl & Human Modified Barrens & $\mathrm{He} / \mathrm{Sls} / \mathrm{Bbg} / \mathrm{He}$ & Human Modified Scrub & $\mathrm{Ffo} / \mathrm{F} / \mathrm{Stoa} / \mathrm{Hen}$ \\
\hline Riverine Gravelly Moist Broadleaf Forest & Fmri/Fi/Fboc/ & Human Modified Barrens & $\mathrm{Hf} / \mathrm{F} / \mathrm{Bbg} / \mathrm{Hcs}$ & Human Modified Scrub & Ffo/F/Stoa/Hcs \\
\hline Riverine Gravelly Moist Mixed Forest & Fhl/F/Fmoas/ & Human Modified Barrens & $\mathrm{Hf} / \mathrm{F} / \mathrm{Bbg} / \mathrm{Hfg}$ & Human Modified Scrub & $\mathrm{Ffo} / \mathrm{F} / \mathrm{Stod} / \mathrm{Hen}$ \\
\hline Riverine Gravelly Moist Mixed Forest & Fhl/F/Fmocws/ & Human Modified Barrens & $\mathrm{Hf} / \mathrm{F} / \mathrm{Bbg} / \mathrm{Hfgp}$ & Human Modified Scrub & $\mathrm{Ffo} / \mathrm{F} / \mathrm{Stod} / \mathrm{Ht}$ \\
\hline Riverine Gravelly Moist Mixed Forest & Fhl/F/Fmosb/ & Human Modified Barrens & $\mathrm{Hf} / \mathrm{F} / \mathrm{Bbg} / \mathrm{Hfgr}$ & Human Modified Scrub & Ffo/F/Stow/Hch \\
\hline Riverine Gravelly Moist Mixed Forest & Fhm/Fi/Fmocws/ & Human Modified Barrens & $\mathrm{Hf} / \mathrm{F} / \mathrm{Bpv} / \mathrm{Hcs}$ & Human Modified Scrub & $\mathrm{Ffo} / \mathrm{F} / \mathrm{Stow} / \mathrm{Hcn}$ \\
\hline Riverine Gravelly Moist Mixed Forest & Fhm/Fi/Fmosb/ & Human Modified Barrens & $\mathrm{Hf} / \mathrm{F} / \mathrm{Bpv} / \mathrm{Hfb}$ & Human Modified Scrub & Ffo/F/Stow/Hcs \\
\hline Riverine Gravelly Moist Mixed Forest & $\mathrm{Fhm} / \mathrm{SI} / \mathrm{Fmosb} /$ & Human Modified Barrens & $\mathrm{Hf} / \mathrm{F} / \mathrm{Bpv} / \mathrm{Hfgp}$ & Human Modified Scrub & Ffo/Sls/Stoa/Hcn \\
\hline Riverine Gravelly Moist Mixed Forest & $\mathrm{Fhm} / \mathrm{Sle} / \mathrm{Fmosb} /$ & Human Modified Barrens & $\mathrm{Hf} / \mathrm{Hm} / \mathrm{Bbg} / \mathrm{Hcs}$ & Human Modified Scrub & $\mathrm{FGp} / \mathrm{F} / \mathrm{Slod} / \mathrm{Hch}$ \\
\hline Riverine Gravelly Moist Mixed Forest & Fmri/F/Fmocws/ & Human Modified Barrens & $\mathrm{Hf} / \mathrm{Hm} / \mathrm{Bbg} / \mathrm{Hfg}$ & Human Modified Scrub & $\mathrm{FGp} / \mathrm{F} / \mathrm{Slod} / \mathrm{Hcn}$ \\
\hline Riverine Gravelly Moist Mixed Forest & Fmri/F/Fmosb/ & Human Modified Barrens & $\mathrm{H} / \mathrm{Hm} / \mathrm{Bbg} / \mathrm{Hfga}$ & Human Modified Scrub & FGp/F/Slod/Hcs \\
\hline Riverine Gravelly Moist Needleleaf Forest & Fhl/F/Fnobw/ & Human Modified Barrens & $\mathrm{Hf} / \mathrm{Hm} / \mathrm{Bbg} / \mathrm{Hfgap}$ & Human Modified Scrub & $\mathrm{FGp} / \mathrm{F} / \mathrm{Slow} / \mathrm{Hch}$ \\
\hline Riverine Gravelly Moist Needleleaf Forest & Fhl/F/Fnows/ & Human Modified Barrens & $\mathrm{Hf} / \mathrm{Hm} / \mathrm{Bbg} / \mathrm{Hfgp}$ & Human Modified Scrub & FGp/F/Slow/Hcn \\
\hline Riverine Gravelly Moist Needleleaf Forest & Fhm/Fi/Fnows/ & Human Modified Barrens & $\mathrm{H} / \mathrm{Hm} / \mathrm{Bbg} / \mathrm{Hfgr}$ & Human Modified Scrub & FGp/F/Slow/Hcs \\
\hline Riverine Gravelly Moist Needleleaf Forest & Fmri/F/Fnows/ & Human Modified Barrens & $\mathrm{Hf} / \mathrm{Hm} / \mathrm{Bbg} / \mathrm{Hfgrp}$ & Human Modified Scrub & FGp/F/Stca/Hch \\
\hline Rivers and Streams & Wert/W/Wb/ & Human Modified Barrens & $\mathrm{Hf} / \mathrm{Sls} / \mathrm{Bbg} / \mathrm{He}$ & Human Modified Scrub & $\mathrm{FGp} / \mathrm{F} / \mathrm{Stca} / \mathrm{Hcn}$ \\
\hline Rivers and Streams & Wrlg/W/WI & Human Modified Barrens & $\mathrm{Hf} / \mathrm{Sue} / \mathrm{Bbg} / \mathrm{Hcs}$ & Human Modified Scrub & $\mathrm{FGp} / \mathrm{F} / \mathrm{Stca} / \mathrm{Hcs}$ \\
\hline Rivers and Streams & Wrin/W/WI & Human Modified Barrens & $\mathrm{H} / \mathrm{Sue} / \mathrm{Bpv} / \mathrm{Hcs}$ & Human Modified Scrub & FGp/F/Stoa/Hch \\
\hline Rivers and Streams & Wrun/W/WI & Human Modified Barrens & $\mathrm{Hf} / \mathrm{Sus} / \mathrm{Bbg} / \mathrm{He}$ & Human Modified Scrub & FGp/F/Stoa/Hcn \\
\hline Coastal Loamy Wet Barrens & $\mathrm{Mta} / \mathrm{F} / \mathrm{Bbg} /$ & Human Modified Barrens & $\mathrm{H} / \mathrm{Sus} / \mathrm{Bpv} / \mathrm{Hen}$ & Human Modified Scrub & $\mathrm{FGp} / \mathrm{F} / \mathrm{Stoa} / \mathrm{Hcs}$ \\
\hline Coastal Loamy Wet Barrens & Mta/F/Bpv/ & Human Modified Barrens & $\mathrm{Hf} / \mathrm{Sus} / \mathrm{Bpv} / \mathrm{Hcs}$ & Human Modified Scrub & FGp/F/Stod/Hch \\
\hline Coastal Loamy Wet Barrens & $\mathrm{Mta} / \mathrm{Fc} / \mathrm{Bbg} /$ & Human Modified Barrens & $\mathrm{MG} / \mathrm{F} / \mathrm{Bbg} / \mathrm{Ht}$ & Human Modified Scrub & $\mathrm{FGp} / \mathrm{F} / \mathrm{Stod} / \mathrm{Hcn}$ \\
\hline Coastal Loamy Wet Barrens & $\mathrm{Mta} / \mathrm{Fc} / \mathrm{Bpv} /$ & Human Modified Barrens & $\mathrm{Ob} / \mathrm{F} / \mathrm{Bpv} / \mathrm{Hcs}$ & Human Modified Scrub & FGp/F/Stod/Hcs \\
\hline Coastal Loamy Wet Barrens & Mta/Ff/Bpv/ & Human Modified Meadow & $\mathrm{Ffo} / \mathrm{F} / \mathrm{Hgmd} / \mathrm{Hcs}$ & Human Modified Scrub & FGp/F/Stow/Hcn \\
\hline Coastal Loamy Wet Barrens & $\mathrm{Mti} / \mathrm{F} / \mathrm{Bbg} /$ & Human Modified Meadow & $\mathrm{FGp} / \mathrm{F} / \mathrm{Bpv} / \mathrm{Hcs}$ & Human Modified Scrub & Fhl/F/Slod/Hcn \\
\hline Coastal Loamy Wet Meadow, brackish & Mta/F/Hfwhh/ & Human Modified Meadow & $\mathrm{FGp} / \mathrm{F} / \mathrm{Hgmd} / \mathrm{Hcn}$ & Human Modified Scrub & Fhl/F/Stca/Hon \\
\hline Coastal Loamy Wet Meadow, brackish & Mta/F/Hgwhg/ & Human Modified Meadow & $\mathrm{FGp} / \mathrm{F} / \mathrm{Hgmd} / \mathrm{Hcs}$ & Human Modified Scrub & Fmob/F/Slod/Hch \\
\hline Coastal Loamy Wet Meadow, brackish & Mta/F/Hgwhsb/ & Human Modified Meadow & $\mathrm{Fmob} / \mathrm{F} / \mathrm{Hgmd} / \mathrm{Hch}$ & Human Modified Scrub & $\mathrm{Fmob} / \mathrm{F} / \mathrm{Stoa} / \mathrm{Hcn}$ \\
\hline Coastal Loamy Wet Meadow, brackish & $\mathrm{Mta} / \mathrm{Ff} / \mathrm{Hfwhh} /$ & Human Modified Meadow & $\mathrm{Fmob} / \mathrm{F} / \mathrm{Hgmd} / \mathrm{Hcs}$ & Human Modified Scrub & Fmob/F/Stod/Hch \\
\hline Coastal Loamy Wet Meadow, brackish & $\mathrm{Mta} / \mathrm{Ff} / \mathrm{Hgwhg} /$ & Human Modified Meadow & $\mathrm{Fmrb} / \mathrm{F} / \mathrm{Hgmd} / \mathrm{Hcs}$ & Human Modified Scrub & $\mathrm{Fmrb} / \mathrm{F} / \mathrm{Slod} / \mathrm{Hch}$ \\
\hline Coastal Loamy Wet Meadow, brackish & Mta/F//Hfwhh/ & Human Modified Meadow & $\mathrm{Fmri} / \mathrm{F} / \mathrm{Hgmd} / \mathrm{Hcn}$ & Human Modified Scrub & Fmrb/F/Slod/Hcs \\
\hline Coastal Loamy Wet Meadow, slightly brackish & Mta/F/Hgwhss/ & Human Modified Meadow & Fmri/F/Hgmd/Hcs & Human Modified Scrub & $\mathrm{Fmrb} / \mathrm{F} / \mathrm{Stca} / \mathrm{Hcn}$ \\
\hline
\end{tabular}




\begin{tabular}{|c|c|c|c|c|c|}
\hline Ecotype & ITU Code & Ecotype & ITU Code & Ecotype & ITU Code \\
\hline Human Modified Scrub & $\mathrm{Fmrb} / \mathrm{F} / \mathrm{Stod} / \mathrm{Hch}$ & Human Modified Scrub & GFo/F/Stod/Hcn & Human Modified Forest & $\mathrm{FGp} / \mathrm{F} / \mathrm{Fbob} / \mathrm{Hch}$ \\
\hline Human Modified Scrub & Fmrb/F/Stod/Hcn & Human Modified Scrub & GFo/F/Stod/Hcs & Human Modified Forest & $\mathrm{FGp} / \mathrm{F} / \mathrm{Fbob} / \mathrm{Hcn}$ \\
\hline Human Modified Scrub & $\mathrm{Fmrb} / \mathrm{F} / \mathrm{Stow} / \mathrm{Hcn}$ & Human Modified Scrub & GFo/Sle/Stca/Hcn & Human Modified Forest & $\mathrm{FGp} / \mathrm{F} / \mathrm{Fbob} / \mathrm{Hcs}$ \\
\hline Human Modified Scrub & Fmri/F/Slod/Hcn & Human Modified Scrub & GFo/Sls/Stca/Hcn & Human Modified Forest & $\mathrm{FGp} / \mathrm{F} / \mathrm{Fboc} / \mathrm{Hcn}$ \\
\hline Human Modified Scrub & Fmri/F/Slod/Hcs & Human Modified Scrub & GFo/Sls/Stca/Hcs & Human Modified Forest & $\mathrm{FGp} / \mathrm{F} / \mathrm{Fmoas} / \mathrm{Hch}$ \\
\hline Human Modified Scrub & Fmri/F/Stca/Hcn & Human Modified Scrub & $\mathrm{GFo} / \mathrm{Xm} / \mathrm{Stoa} / \mathrm{Hcn}$ & Human Modified Forest & $\mathrm{FGp} / \mathrm{F} / \mathrm{Fmoas} / \mathrm{Hcn}$ \\
\hline Human Modified Scrub & Fmri/F/Stoa/Hcn & Human Modified Scrub & Gmo/F/Slod/Hch & Human Modified Forest & $\mathrm{FGp} / \mathrm{F} / \mathrm{Fmosb} / \mathrm{Hch}$ \\
\hline Human Modified Scrub & Fmri/F/Stow/Hcn & Human Modified Scrub & $\mathrm{Gmo} / \mathrm{F} / \mathrm{Slod} / \mathrm{Hcn}$ & Human Modified Forest & $\mathrm{FGp} / \mathrm{F} / \mathrm{Fmosb} / \mathrm{Hcn}$ \\
\hline Human Modified Scrub & $\mathrm{GFc} / \mathrm{F} / \mathrm{Slod} / \mathrm{Hch}$ & Human Modified Scrub & $\mathrm{Gmo} / \mathrm{F} / \mathrm{Slod} / \mathrm{Hcs}$ & Human Modified Forest & FGp/F/Fnows/Hcn \\
\hline Human Modified Scrub & $\mathrm{GFc} / \mathrm{F} / \mathrm{Slod} / \mathrm{Hcn}$ & Human Modified Scrub & $\mathrm{Gmo} / \mathrm{F} / \mathrm{Slow} / \mathrm{Hcn}$ & Human Modified Forest & Fmob/F/Fboc/Hch \\
\hline Human Modified Scrub & GFc/F/Slod/Hcs & Human Modified Scrub & $\mathrm{Gmo} / \mathrm{F} / \mathrm{Stca} / \mathrm{Hcn}$ & Human Modified Forest & Fmob/F/Fmocws/Hcn \\
\hline Human Modified Scrub & GFc/F/Slow/Hcn & Human Modified Scrub & $\mathrm{Gmo} / \mathrm{F} / \mathrm{Stcd} / \mathrm{Hcn}$ & Human Modified Forest & Fmob/F/Fmosb/Hch \\
\hline Human Modified Scrub & $\mathrm{GFc} / \mathrm{F} / \mathrm{Stca} / \mathrm{Hcn}$ & Human Modified Scrub & $\mathrm{Gmo} / \mathrm{F} / \mathrm{Stoa} / \mathrm{Hcn}$ & Human Modified Forest & Fmob/F/Fnobw/Hch \\
\hline Human Modified Scrub & $\mathrm{GFc} / \mathrm{F} / \mathrm{Stca} / \mathrm{Hcs}$ & Human Modified Scrub & Gmo/F/Stoa/Hcs & Human Modified Forest & Fmob/F/Fnobw/Hcn \\
\hline Human Modified Scrub & $\mathrm{GFc} / \mathrm{F} / \mathrm{Stoa} / \mathrm{Hcn}$ & Human Modified Scrub & $\mathrm{Gmo} / \mathrm{F} / \mathrm{Stod} / \mathrm{Hcn}$ & Human Modified Forest & $\mathrm{Fmrb} / \mathrm{F} / \mathrm{Fbob} / \mathrm{Hcn}$ \\
\hline Human Modified Scrub & $\mathrm{GF} / \mathrm{F} / \mathrm{Stoa} / \mathrm{Hcs}$ & Human Modified Scrub & $\mathrm{Gmo} / \mathrm{F} / \mathrm{Stod} / \mathrm{Hcs}$ & Human Modified Forest & $\mathrm{Fmrb} / \mathrm{F} / \mathrm{Fboc} / \mathrm{Hcn}$ \\
\hline Human Modified Scrub & $\mathrm{GFc} / \mathrm{F} / \mathrm{Stod} / \mathrm{Hcn}$ & Human Modified Scrub & $\mathrm{Gmo} / \mathrm{Sle} / \mathrm{Slod} / \mathrm{Hch}$ & Human Modified Forest & $\mathrm{Fmri} / \mathrm{F} / \mathrm{Fboc} / \mathrm{Hcn}$ \\
\hline Human Modified Scrub & $\mathrm{GFc} / \mathrm{F} / \mathrm{Stod} / \mathrm{Hcs}$ & Human Modified Scrub & $\mathrm{Gmo} / \mathrm{Sle} / \mathrm{Slod} / \mathrm{Hcn}$ & Human Modified Forest & Fmri/F/Fboc/Hcs \\
\hline Human Modified Scrub & GFc/F/Stow/Hch & Human Modified Scrub & $\mathrm{Gmo} / \mathrm{Sle} / \mathrm{Stca} / \mathrm{Hcn}$ & Human Modified Forest & $\mathrm{GFc} / \mathrm{F} / \mathrm{Fbob} / \mathrm{Hcn}$ \\
\hline Human Modified Scrub & $\mathrm{GF} / \mathrm{F} / \mathrm{Stow} / \mathrm{Hcn}$ & Human Modified Scrub & $\mathrm{Gmo} / \mathrm{Sle} / \mathrm{Stoa} / \mathrm{Hcn}$ & Human Modified Forest & $\mathrm{GFc} / \mathrm{F} / \mathrm{Fmosb} / \mathrm{Hcn}$ \\
\hline Human Modified Scrub & $\mathrm{GFc} / \mathrm{Sle} / \mathrm{Slod} / \mathrm{Hcn}$ & Human Modified Scrub & $\mathrm{Gmo} / \mathrm{Sle} / \mathrm{Stod} / \mathrm{Hcn}$ & Human Modified Forest & GFc/F/Fnobs/Hcn \\
\hline Human Modified Scrub & GFc/Sle/Slod/Hcs & Human Modified Scrub & Gmo/Sle/Stow/Hcn & Human Modified Forest & GFc/F/Fnobw/Hcn \\
\hline Human Modified Scrub & $\mathrm{GFc} / \mathrm{Sle} / \mathrm{Stod} / \mathrm{Hch}$ & Human Modified Scrub & $\mathrm{Gmo} / \mathrm{SIn} / \mathrm{Stca} / \mathrm{Hcs}$ & Human Modified Forest & GFc/F/Fnows/Hcn \\
\hline Human Modified Scrub & $\mathrm{GFc} / \mathrm{SIn} / \mathrm{Stca} / \mathrm{Hcn}$ & Human Modified Scrub & $\mathrm{Gmo} / \mathrm{Sls} / \mathrm{Slod} / \mathrm{Hcn}$ & Human Modified Forest & GFc/SIc/Fmosb/Hcn \\
\hline Human Modified Scrub & $\mathrm{GFc} / \mathrm{S} \ln / \mathrm{Stow} / \mathrm{Hcn}$ & Human Modified Scrub & $\mathrm{Gmo} / \mathrm{Sls} / \mathrm{Slod} / \mathrm{Hcs}$ & Human Modified Forest & GFc/Sle/Fmosb/Hcn \\
\hline Human Modified Scrub & GFc/Sls/Slod/Hcs & Human Modified Scrub & $\mathrm{Gmo} / \mathrm{Sls} / \mathrm{Stca} / \mathrm{Hcn}$ & Human Modified Forest & GFc/Sle/Fnobw/Hcn \\
\hline Human Modified Scrub & GFc/Sls/Slow/Hcn & Human Modified Scrub & $\mathrm{Gmo} / \mathrm{Sls} / \mathrm{Stod} / \mathrm{Hcn}$ & Human Modified Forest & GFk/F/Fbob/Hcn \\
\hline Human Modified Scrub & GFc/Sls/Stoa/Hcn & Human Modified Scrub & Gmo/Sue/Stoa/Hcn & Human Modified Forest & GFk/F/Fbob/Hcs \\
\hline Human Modified Scrub & GFc/Sls/Stod/Hch & Human Modified Scrub & Gmo/Sus/Stca/Hcn & Human Modified Forest & GFk/F/Fboba/Hcn \\
\hline Human Modified Scrub & GFc/Sls/Stow/Hch & Human Modified Scrub & Gmo/Sus/Stoa/Hcn & Human Modified Forest & $\mathrm{GFk} / \mathrm{F} / \mathrm{Fmosb} / \mathrm{Hch}$ \\
\hline Human Modified Scrub & $\mathrm{GFc} / \mathrm{Xm} / \mathrm{Slod} / \mathrm{Hcn}$ & Human Modified Scrub & $\mathrm{Gmo} / \mathrm{Xm} / \mathrm{Slod} / \mathrm{Hcn}$ & Human Modified Forest & $\mathrm{GFk} / \mathrm{F} / \mathrm{Fmosb} / \mathrm{Hcn}$ \\
\hline Human Modified Scrub & GFk/B/Slod/Hcn & Human Modified Scrub & $\mathrm{Gmo} / \mathrm{Xm} / \mathrm{Slod} / \mathrm{Hcs}$ & Human Modified Forest & GFk/F/Fnows/Hcn \\
\hline Human Modified Scrub & GFk/F/Slod/Hch & Human Modified Scrub & $\mathrm{Gmo} / \mathrm{Xm} / \mathrm{Slod} / \mathrm{Ht}$ & Human Modified Forest & GFk/Sle/Fbob/Hon \\
\hline Human Modified Scrub & GFk/F/Slod/Hcn & Human Modified Scrub & $\mathrm{Gmo} / \mathrm{Xm} / \mathrm{Stca} / \mathrm{Hch}$ & Human Modified Forest & GFk/Sle/Fmosb/Hcn \\
\hline Human Modified Scrub & GFk/F/Slod/Hcs & Human Modified Scrub & $\mathrm{Gmo} / \mathrm{Xm} / \mathrm{Stca} / \mathrm{Hcn}$ & Human Modified Forest & GFk/Sle/Fnows/Hch \\
\hline Human Modified Scrub & GFk/F/Stca/Hch & Human Modified Scrub & $\mathrm{Gmo} / \mathrm{Xm} / \mathrm{Stca} / \mathrm{Hcs}$ & Human Modified Forest & GFk/Sle/Fnows/Hcn \\
\hline Human Modified Scrub & $\mathrm{GFk} / \mathrm{F} / \mathrm{Stca} / \mathrm{Hcn}$ & Human Modified Scrub & Gmo/Xm/Stoa/Hch & Human Modified Forest & GFk/Sls/Fnows/Hcn \\
\hline Human Modified Scrub & GFk/F/Stca/Hcs & Human Modified Scrub & $\mathrm{Gmo} / \mathrm{Xm} / \mathrm{Stoa} / \mathrm{Hcn}$ & Human Modified Forest & GFk/Xm/Fboa/Hcs \\
\hline Human Modified Scrub & GFk/F/Stoa/Hcn & Human Modified Scrub & Gmo/Xm/Stoa/Hcs & Human Modified Forest & $\mathrm{GFk} / \mathrm{Xm} / \mathrm{Fmosb} / \mathrm{Hcn}$ \\
\hline Human Modified Scrub & GFk/F/Stoa/Hcs & Human Modified Scrub & $\mathrm{Gmo} / \mathrm{Xm} / \mathrm{Stod} / \mathrm{Hcn}$ & Human Modified Forest & GFk/Xm/Fnows/Hch \\
\hline Human Modified Scrub & GFk/F/Stod/Hch & Human Modified Scrub & Gmo/Xm/Stow/Hch & Human Modified Forest & GFk/Xm/Fnows/Hcn \\
\hline Human Modified Scrub & $\mathrm{GFk} / \mathrm{F} / \mathrm{Stod} / \mathrm{Hcn}$ & Human Modified Scrub & $\mathrm{Gmo} / \mathrm{Xm} / \mathrm{Stow} / \mathrm{Hcn}$ & Human Modified Forest & GFo/F/Fbob/Hcn \\
\hline Human Modified Scrub & $\mathrm{GFk} / \mathrm{F} / \mathrm{Stod} / \mathrm{Hcs}$ & Human Modified Scrub & $\mathrm{He} / \mathrm{F} / \mathrm{Stca} / \mathrm{He}$ & Human Modified Forest & $\mathrm{Gmo} / \mathrm{C} / \mathrm{Fmosb} / \mathrm{Hcn}$ \\
\hline Human Modified Scrub & $\mathrm{GFk} / \mathrm{F} / \mathrm{Stod} / \mathrm{Ht}$ & Human Modified Scrub & $\mathrm{He} / \mathrm{Hm} / \mathrm{Slod} / \mathrm{He}$ & Human Modified Forest & Gmo/F/Fbob/Hcn \\
\hline Human Modified Scrub & GFk/F/Stow/Hch & Human Modified Scrub & $\mathrm{He} / \mathrm{Hm} / \mathrm{Stoa} / \mathrm{He}$ & Human Modified Forest & $\mathrm{Gmo} / \mathrm{F} / \mathrm{Fmosb} / \mathrm{Hcn}$ \\
\hline Human Modified Scrub & GFk/Sle/Slod/Hch & Human Modified Scrub & $\mathrm{He} / \mathrm{Hm} / \mathrm{Stod} / \mathrm{He}$ & Human Modified Forest & $\mathrm{Gmo} / \mathrm{SIn} / \mathrm{Fbob} / \mathrm{Hcn}$ \\
\hline Human Modified Scrub & GFk/Sle/Slod/Hcn & Human Modified Scrub & $\mathrm{Hf} / \mathrm{Hm} / \mathrm{Slod} / \mathrm{Hf}$ & Human Modified Forest & $\mathrm{Gmo} / \mathrm{SIn} / \mathrm{Fnobw} / \mathrm{Hcn}$ \\
\hline Human Modified Scrub & GFk/Sle/Stca/Hcn & Human Modified Scrub & MG/F/Slod/Hch & Human Modified Forest & $\mathrm{Gmo} / \mathrm{Sls} / \mathrm{Fbob} / \mathrm{Hcn}$ \\
\hline Human Modified Scrub & GFk/Sle/Stca/Hcs & Human Modified Scrub & $\mathrm{MG} / \mathrm{F} / \mathrm{Slod} / \mathrm{Hcn}$ & Human Modified Forest & $\mathrm{Gmo} / \mathrm{Xm} / \mathrm{Fbob} / \mathrm{Hcn}$ \\
\hline Human Modified Scrub & $\mathrm{GFk} / \mathrm{Sln} / \mathrm{Stca} / \mathrm{Hcn}$ & Human Modified Scrub & MG/F/Stca/Hch & Human Modified Forest & $\mathrm{Gmo} / \mathrm{Xm} / \mathrm{Fmosb} / \mathrm{Hcn}$ \\
\hline Human Modified Scrub & GFk/SIn/Stod/Hcn & Human Modified Scrub & MG/F/Stod/Hch & Human Modified Forest & $\mathrm{Gmo} / \mathrm{Xm} /$ Fnows $/ \mathrm{Hcn}$ \\
\hline Human Modified Scrub & GFk/Sls/Slod/Hcn & Human Modified Scrub & $\mathrm{MG} / \mathrm{F} / \mathrm{Stod} / \mathrm{Ht}$ & Human Modified Waterbodies & Wh/W/W/Hwd \\
\hline Human Modified Scrub & GFk/Sls/Stca/Hcs & Human Modified Scrub & MG/F/Stow/Hch & Human Modified Waterbodies & Wh/W/W/Hwe \\
\hline Human Modified Scrub & GFk/SIs/Stoa/Hcn & Human Modified Scrub & MG/Sle/Slod/Hcn & Human Modified Complex & $\mathrm{Ff} / \mathrm{F} / \mathrm{DC} / \mathrm{DC}$ \\
\hline Human Modified Scrub & GFk/Sue/Stca/Hcn & Human Modified Scrub & MG/Sls/Stow/Hcn & Human Modified Complex & FGp/F/DC/DC \\
\hline Human Modified Scrub & GFk/Xm/Slod/Hch & Human Modified Scrub & $\mathrm{MG} / \mathrm{Xm} / \mathrm{Slod} / \mathrm{Ht}$ & Human Modified Complex & $\mathrm{FGp} / \mathrm{Hm} / \mathrm{DC} / \mathrm{DC}$ \\
\hline Human Modified Scrub & GFk/Xm/Slod/Hcn & Human Modified Scrub & $\mathrm{MG} / \mathrm{Xm} / \mathrm{Stow} / \mathrm{Hcn}$ & Human Modified Complex & $\mathrm{Fmri} / F / D C / D C$ \\
\hline Human Modified Scrub & $\mathrm{GFk} / \mathrm{Xm} / \mathrm{Slod} / \mathrm{Ht}$ & Human Modified Scrub & $\mathrm{Ob} / \mathrm{F} / \mathrm{Slobb} / \mathrm{Ht}$ & Human Modified Complex & GFC/F/DC/DC \\
\hline Human Modified Scrub & GFk/Xm/Stca/Hcn & Human Modified Scrub & $\mathrm{Ob} / \mathrm{F} / \mathrm{Slod} / \mathrm{Hcn}$ & Human Modified Complex & GFk/F/DC/DC \\
\hline Human Modified Scrub & GFk/Xm/Stoa/Hch & Human Modified Scrub & $\mathrm{Ob} / \mathrm{F} / \mathrm{Slod} / \mathrm{Ht}$ & Human Modified Complex & $\mathrm{GFk} / \mathrm{Xm} / \mathrm{DC} / \mathrm{DC}$ \\
\hline Human Modified Scrub & GFk/Xm/Stoa/Hcn & Human Modified Forest & $\mathrm{Ffo} / \mathrm{F} / \mathrm{Fbob} / \mathrm{Hcn}$ & Human Modified Complex & $\mathrm{Gmo} / \mathrm{F} / \mathrm{DC} / \mathrm{DC}$ \\
\hline Human Modified Scrub & $\mathrm{GFk} / \mathrm{Xm} / \mathrm{Stod} / \mathrm{Hcn}$ & Human Modified Forest & $\mathrm{Ffo} / \mathrm{F} / \mathrm{Fbob} / \mathrm{Hcs}$ & Human Modified Complex & $\mathrm{Gmo} / \mathrm{Xm} / \mathrm{DC} / \mathrm{DC}$ \\
\hline Human Modified Scrub & GFk/Xm/Stod/Hcs & Human Modified Forest & $\mathrm{Ffo} / \mathrm{F} / \mathrm{Fmosb} / \mathrm{Hcn}$ & Human Modified Complex & $\mathrm{H} / \mathrm{C} / \mathrm{DC} / \mathrm{DC}$ \\
\hline Human Modified Scrub & GFo/F/Slod/Hcn & Human Modified Forest & Ffo/F/Fnows/Hen & Human Modified Complex & $\mathrm{Hf} / \mathrm{F} / \mathrm{DC} / \mathrm{DC}$ \\
\hline Human Modified Scrub & GFo/F/Slod/Hcs & Human Modified Forest & $\mathrm{FGp} / \mathrm{F} / \mathrm{Fboa} / \mathrm{Hcn}$ & Human Modified Complex & $\mathrm{H} / \mathrm{Hm} / \mathrm{DC} / \mathrm{DC}$ \\
\hline
\end{tabular}


Appendix D. Vascular plants found on Fort Richardson, south-central Alaska, 2001 (derived from Lichvar et al. 1997).

Achillea millefolium $\mathrm{L}$.

Achillea ptarmica $\mathrm{L}$.

Achillea sibirica Ledeb.

Acomastylis rossii (R. Br.) E. Greene [=Geum rossii (R. Br.) Ser. ex DC.]

Aconitum delphinifolium DC.

Aconitum delphinifolium DC. ssp. paradoxicum (Reichb.) Maguire \& Hult.

Actaea rubra (Ait.) Willd.

Adoxa moschatellina $\mathrm{L}$.

Agrostis scabra Willd.

Allium schoenoprasum L.

Alnus sinuata (Regel) Rydb. [=A. crispa (Ait.) Pursh ssp. sinuata (Regel) Hult.]

Alnus tenuifolia Nutt. $[=$ A. incana $($ L.) Moench ssp. tenuifolia (Nutt.) Breitung]

Alnus viridis Villar ssp. crispa (Ait.) Loeve \& Loeve $[=$ A. crispa (Ait.) Pursh ssp. crispa]

Alopecuris aequalis Sobol.

Alopecuris alpinus Smith

Amaranthus retroflexus $\mathrm{L}$.

Amelanchier alnifolia (Nutt.) Nutt.

Andromeda polifolia $\mathrm{L}$.

Anemone multifida Poir. var. saxicola B. Boivan

Anemone narcissiflora L. ssp. villosissima (DC.) Hult.

Anemone narcissiflora L. var. monantha DC.

Anemone parviflora Michx.

Anemone richardsonii Hock.

Angelica genuflexa Nutt.

Angelica lucida E. Nels.

Antennaria alpina (L.) Gaertn.

Antennaria friesiana (Trautv.) Ekman

Antennaria friesiana (Trautv.) Ekman ssp. alaskana (Malte) Hult.

Antennaria monocephala DC.

Antennaria rosea E. Greene ssp. pulvinata (E. Greene) Bayer

Antennaria rosea (D.C. Eaton) E. Greene

Anthemis cotula L.

Anthemis tinctoria L.

Aphragmus eschscholtzianus Andrz.

Aquilegia formosa Fisch.

Arabis hirsuta (L.) Scop. ssp. eschscholtziana (Andrz.) Hult.

Arabis holboellii Hornem.

Arabis lyrata L. ssp. kamchatica (Fisch.) Hult.

Arctagrostis latifolia (R. Br.) Griseb.

Arctagrostis poaeoides Nash

Arctogrostis latifolia (R. Br.) Griseb. var. arundinacea (Trin.)

Griseb.

Arctogrostis latifolia (R. Br.) Griseb. var. latifolia

Arctostaphylos uva-ursi (L.) Sprengel

Arctous alpina (L.) Niedenzu [=Arctostaphylos alpina (L.)

Spreng.]

Arctous rubra (Rehd. \& Wilson) Nakai [=Arctostphylos rubra (Rehd. \& Wilson) Fern.]

Armeria maritima (Mill.) Willd. ssp. arctica (Cham.) Hult. Arnica griscomii Fern. ssp. frigida (C. Meyer ex Iljin) S. J. Wolf Arnica latifolia Bong.

Arnica lessingii Greene

Arnica ovata E. Greene

Artemisia arctica Less.

Artemisia tilesii Ledeb.

Aster junciformis Rydb.

Aster sibiricus $\mathrm{L}$.

Astragalus alpinus L.

Astragalus alpinus L. ssp. alpinus
Astragalus polaris Benth.

Astragalus umbellatus Bunge

Athyrium filix-femina (L.) Roth

Atriplex gmelini C.A. Meyer

Avena fatua L.

Barbarea orthoceras Ledeb.

Beckmannia erucaeformis (L.) Host ssp. baicalensis (Kusn.) Hult.

Betula glandulosa Michx.

Betula hybrids

Betula kenaica Evans

Betula nana L. ssp. exilis (Sukatsch.) Hult. ${ }^{1}$

Betula occidentalis Hook. ${ }^{1}$

Betula papyrifera Marshall

Bistorta vivipara (L.) Gray [=Polygonum viviparum L.]

Boschniakia rossica (Cham \& Schldl.) B. Fedtsch.

Botrichium boreale (E.Fries) Milde (=Botrichium pinnatum $\mathrm{H}$. St. John In: FNA ${ }^{2}$ )

Botrichium lanceolatum (Gmel.) Angstr.

Botrichium lunaria (L.) Sw.

Brassica rapa $\mathrm{L}$.

Bromopsis inermis (Leyss.) Holub [=Bromus inermis Leyss.]

Bromus tectorum $\mathrm{L}$.

Calamagrostis canadensis (Michx.) Beauv.

Calamagrostis deschampsioides Trin.

Calamagrostis inexpansa Gray

Calamagrostis lapponica (Wahlenb.) Hartman. F.

Calamagrostis nutkaensis (C. Presl) Steudel

Calamagrostis purpurascens $\mathrm{R}$. Br. ssp. purpurascens ${ }^{1}$

Callitriche verna $\mathrm{L}$. emend. Lonnr.

Caltha palustris L. ssp. asarifolia (DC.) Hult.

Campanula lasiocarpa Cham.

Campanula rotundifolia $\mathrm{L}$.

Campanula uniflora $\mathrm{L}$.

Capsella bursa-pastoris (L.) Medic.

Capsella rubella Reut.

Cardamine bellidifolia $\mathrm{L}$.

Cardamine pratensis L. ssp. angustifolia (Hook.) O.E. Schultz

Cardamine umbellata Greene

Carex aquatilis Wahlenb. ssp. aquatilis

Carex atrosquama Mackenzie

Carex bigelowii Torr.

Carex buxbaumii Wahlenb.

Carex canescens $\mathrm{L}$.

Carex chordorrhiza Ehrh.

Carex circinnata C. A. Mey.

Carex deweyana Schwein.

Carex diandra Schrank

Carex dioica L. ssp. gynocrates (Wormsk.) Hult.

Carex garberi Fern. ssp. bifaria (Fern.) Hult.

Carex gmelinii Hook. \& Arn.

Carex kelloggii W. Boott

Carex lachenalii Schkuhr.

Carex lasiocarpa Ehrh. ssp. americana (Fern.) Hult.

Carex leptalea Wahlenb.

Carex limosa $\mathrm{L}$.

Carex livida (Wahlenb.) Willd.

Carex loliacea $\mathrm{L}$.

Carex lyngbyaei Hornem.

Carex mackenziei V. Krecz.

Carex macloviana Urv.

Carex macrochaeta C.A. Mey.

Carex magellanica Lam. ssp. irrigua (Wahlenb.) Hult.

Carex media $\mathrm{R}$. Br.

Carex membranacea Hook. 
Carex mertensii Prescott

Carex microchaeta Holm.

Carex microchaeta Holm. ssp. nesophila (Holm.) D. Murray

Carex micropoda C.A. Meyer $[=C$. pyrenaica Wahlenb. ssp. micropoda (C. A. Meyer) Hult.]

Carex nigricans C.A. Meyer

Carex obtusata Lilj.

Carex oederi Retz.

Carex pauciflora Lightf.

Carex pluriflora Hult.

Carex podocarpa C.B. Clarke

Carex praticola $\mathrm{Rydb}$.

Carex ramenskii Kom.

Carex rariflora (Wahlenb.) Smith

Carex rostrata Stokes

Carex rotundata Wahlenb.

Carex saxatilis $\mathrm{L}$.

Carex scirpoidea Michx.

Carex spectabilis Dewey

Carex tenuiflora Wahlenb.

Carex utriculata F. Boott

Carex vaginata Tausch

Cassiope lycopodioides (Pall.) D. Don

Cassiope stelleriana (Pall.) DC.

Cassiope tetragona (L.) D. Don

Castilleja unalaschcensis (Cham. \& Schlecht.) Malte

Cerastium arvense $\mathrm{L}$.

Cerastium beeringianum Cham. \& Schlecht. var. beeringianum

Cerastium fontanum Baumg.

Chamaedaphne calyculata (L.) Moench

Chenopodium album $\mathrm{L}$.

Chrysanthemum arcticum $\mathrm{L}$.

Chrysanthemum leucanthemum $\mathrm{L}$.

Chrysosplenium tetrandrum (Lund) T. Fries

Cicuta douglasii (DC.) J. Coulter \& Rose

Cicuta virosa L. [=C. mackenzieana Raup]

Circaea alpina $\mathrm{L}$.

Claytonia sarmentosa C. Meyer

Coeloglossum viride (L.) Hartm. ssp. bracteatum (Muhl.) Hult.

Comarum palustre L. [=Potentilla palustris (L.) Scop.]

Conioselinum pacificum (S. Wats.) Coult. \& Rose $[=C$. chinense (L.) BSP.]

Corallorrhiza trifida Chatel.

Cornus canadensis $\mathrm{L}$.

Cornus suecica $\mathrm{L}$.

Corydalis pauciflora (Steph.) Pers.

Corydalis sempervirens (L.) Pers.

Crepis elegans Hook.

Crepis nana Richards.

Crepis tectorum $\mathrm{L}$.

Cryptogramma acrostichoides $\mathrm{R}$. Br. $[=C$. crispa $(\mathrm{L}$.) R. Br. var. acrostichoides (R. Br.) Clarke

Cystopteris fragilis (L.) Bernh.

Cystopteris montana (Lam.) Bernh.

Dactylis glomerata L.

Delphinium glaucum $\mathrm{S}$. Wats.

Deschampsia caespitosa (L.) P. Beauv. ssp. caespitosa

Descurainia sophioides (Fisch.) O.E. Shultz

Diapensia lapponica L.

Dodecatheon pulchellum (Raf.) Merr.

Douglasia alaskana (Cov. \& Stand. ex Hult.) S. Kelso

[=Androsace alaskana Cov. \& Stand.]

Draba alpina $\mathrm{L}$.

Draba aurea Vahl

Draba borealis DC.

Draba cana $\mathrm{Rydb} .[=$ D. lanceolata Royle In: Hulten)

Draba crassifolia Graham

Draba fladzinensis Wulf
Draba glabella Pursh

Draba lactea Adams

Draba lonchocarpa Rydb.

Draba longipes Raup

Draba nivalis Liljebl.

Draba ruaxes Payson \& H. St. John

Draba stenoloba Ledeb.

Draba stenopetala Trautv.

Drocera anglica Huds.

Drocera rotundifolia $\mathrm{L}$.

Dryas alaskensis Pors. [=D. octopetala L. ssp. alaskensis (Pors.) Hult.]

Dryas drummondii Richards.

Dryas integrifolia Vahl.

Dryas octopetala $\mathrm{L}$

Dryopteris dilatata (Hoffm.) A.Gray

Dryopteris fragrans (L.) Schott

Eleocharis kamtschatica (C.A. Meyer) V. Komarov

Eleocharis palustris (L.) Roem. \& Schult.

Eleocharis quinquefolia (F. Hartmann) O. Schwarz

Elymus alaskanus (Scribn. \& Merr.) A. Loeve ssp. alaskanus [=Agropyron violaceum (Hornem.) Lange]

Elymus glaucus Buckley

Elymus sibiricus L.

Elymus trachycaulis (Link) Gould ex Shinners ssp. andinus (Schribner \& Smith) A

Elymus trachycaulis (Link) Gould ex Shinners ssp. novae-angliae (Scribn.) Tzvelev [=Agropyron pauciflorum (Schwein.) Hitchc. ssp. novae-angliae (Scribn.) Meldris]

Elytrigia repens (L.) Nevski [=Agropyron repens (L.) Beauv.]

Empetrum hermaphroditum (Lange) Hagerup $[=$ E. nigrum L. ssp. hermaphroditum (Lange) Boecher]

Empetrum nigrum $\mathrm{L}$.

Epilobium anagallidifolium Lam.

Epilobium angustifolium $\mathrm{L}$.

Epilobium ciliatum Raf. ssp. glandulosum (Lehm.) Hoch \& Raven [=E.glandulosum Lehm.]

Epilobium hornemannii Reichb. ssp. hornemannii

Epilobium latifolium $\mathrm{L}$.

Epilobium palustre $\mathrm{L}$.

Equisetum arvense $\mathrm{L}$.

Equisetum fluviatile L. ampl. Ehrh.

Equisetum palustre $\mathrm{L}$.

Equisetum pratense L.

Equisetum scirpoides Michx.

Equisetum silvaticum L.

Equisetum variegatum Schleich.

Erigeron acris L.

Erigeron humilis Graham

Erigeron peregrinus (Pursh) Greene

Erigeron purpuratus Greene

Eriophorum angustifolium Honck. ssp. subarcticum (V. Vassiljev) Hult.

Eriophorum gracile Koch

Eriophorum russeolum Fries

Eriophorum russeolum Fries var. albidum W. Nyl.

Eriophorum scheuchzeri Hoppe

Eriophorum viridi-carinatum (Englem.) Fern.

Erucastrum gallicum (Willd.) O. E. Schulz [=Brassica erucastrum $]$

Erysimum cheiranthoides L.

Erysimum cheiranthoides L. ssp. altum Ahti

Euphrasia disjuncta Fern \& Wieg.

Eutrema edwardsii R. Br.

Festuca altaica Trin.

Festuca brachyphylla Schult. ${ }^{1}$

Festuca brevissima Yurtsev

Festuca rubra L.

Festuca vivipara (L.) Smith 
Fragaria chiloensis (L.) Duchesne

Fritillaria camschatcensis (L.) Ker-Gawl.

Galeopsis bifida Boem.

Galium boreale L.

Galium trifidum L. ssp. trifidum

Galium triflorum Michx.

Gastrolychnis apetala (L.) Tolm \& Koz. [=Melandrium apetalum (L.) Fenzl.]

Gentiana glauca Pallas

Gentianella amarella $(\mathrm{L}$.$) Boerner [=$ Gentiana amarella $\mathrm{L}$. ssp. acuta (Michx.) Hult.]

Gentianella propinqua (Richards.) Gillet var. propinqua $[=$ Gentiana propinqua Richards. ssp. propinqua $]$

Geocaulon lividum (Richards.) Fern.

Geranium erianthum DC.

Geranium pusillum Burm.

Geum macrophyllum Willd. ssp. macrophyllum

Geum perincisum $\mathrm{Rydb}$. [=G. macrophyllum Willd. ssp. perincisum (Rydb.) Raup.]

Glaux maritima L.

Glyceria borealis (Nash) Batch.

Glyceria striata (Lam.) A. Hitchc. ssp. stricta (Scribn.) Hult.

Goodyera repens (L.) R. Br. var. ophioides Fern.

Gymnocarpium dryopteris (L.) Newm.

Hammarbya paludosa (L.) Ktze.

Hedysarum alpinum $\mathrm{L}$.

Helianthus anuus $\mathrm{L}$.

Heracleum lanatum Michx.

Heuchera glabra Willd.

Hieracium triste Willd.

Hieracium scabriusculum Schwein. ${ }^{1}$

Hierochloe alpina (Sw.) Roem. \& Schult.

Hierochloe odorata (L.) P. Beauv.

Hippuris montana Ledeb.

Hippuris tetraphylla L.F.

Hippuris vulgaris $\mathrm{L}$.

Hordeum brachyantherum Nevski

Hordeum jubatum $\mathrm{L}$.

Huperzia selago (L.) C. Martius [ $=$ H. haleakalae (Brackenridge) Holub In: $\mathrm{FNA}^{2}$ ]

Huperzia selago (L.) C. Martius ssp. chinense (C.Chr.) Loeve \& Loeve [=Lycopodium selago L. ssp. chinense (C. Chr.) Hult.; $=H$. myoshiana (Makino) Ching In: $\left.\mathrm{FNA}^{2}\right]$

Impatiens noli-tangere $\mathrm{L}$.

Iris setosa Pall. ssp. setosa

Isoetes echinospora Durieu

Juncus alpinus Villers

Juncus biglumis L.

Juncus bufonius $\mathrm{L}$.

Juncus castaneus Smith

Juncus castaneus Sm. ssp. castaneus

Juncus castaneus Sm. ssp. leucochlamys (Zinz.) Hult.

Juncus drummondii E. M

Juncus ensifolius Wikstrom

Juncus mertensianus Bong.

Juncus stygius L. ssp. americanus (Buchenau) Hult.

Juncus triglumis $\mathrm{L}$.

Juniperus communis $\mathrm{L}$.

Lathyrus palustris L. ssp. pilosus (Cham.) Hult.

Ledum groenlandicum Oeder $[=L$. palustre L. ssp. groenlandicum (Oeder) Hult.]

Ledum palustre L. ssp. decumbens (Ait.) Hult.

Lemna minor $\mathrm{L}$.

Lepidium densiflorum Schrad.

Leptarrhena pyrolifolia (D. Don) Ser.

Leymus mollis (Trin.) Hara ssp. mollis $[=$ Elymus arenarius L. ssp. mollis (Trin.) Hult.]

Ligusticum scoticum L. ssp. hultenii (Fern.) Cald. \& Tayl.
Linaria vulgaris Mill.

Linnaea borealis $\mathrm{L}$.

Listera cordata (L.) R. Br.

Lloydia serotina (L.) Rchb.

Loiseleuria procumbens (L.) Desv.

Lolium multiflorum Lam.

Luetkea pectinata (Pursh) Ktze.

Lupinus nootkatensis Donn

Lupinus polyphyllus Lindl.

Luzula arcuata (Wahlenb.) Sw.

Luzula arcuata (Wahlenb.) Sw. ssp. unalaschensis (Buchenau)

Hult.

Luzula confusa Lindeb.

Luzula multiflora (Retz.) Lej. var. frigida (Buchenau) Hult.

Luzula parviflora (Ehrh.) Desv.

Luzula spicata (L.) DC.

Luzula wahlenbergii Rupr.

Lychnis chalcedonica $\mathrm{L}$.

Lycopodium alpinum L. [=Diphasiastrum alpinum (L.) Holub In: $\mathrm{FNA}^{2}$ ]

Lycopodium annotinum $\mathrm{L}$.

Lycopodium clavatum L. ssp. monostachyon (Grev. \& Hook.) Sel. $\left[=\right.$ L. lagopus (Laest. ex C. Hartman) In: $\left.\mathrm{FNA}^{2}\right]$

Lycopodium complanatum L. [=Diphasiastrum complanatum (L.) Holub In: $\mathrm{FNA}^{2}$ ]

Lycopodium sabinaefolium Willd. var. sitchense (Rupt.)Fern. [=Diphasiastrum sitchense (Ruprecht) Holub In: NNA $\left.^{2}\right]$

Lysimachia thyrsiflora $\mathrm{L}$.

Malaxis monophylla (L.) Sw. var. brachypoda (A. Gray) Morris \& Ames

Matricaria matricarioides (Less.) Porter

Matteuccia struthiopteris (L.) Tod.

Medicago falcata $\mathrm{L}$.

Medicago sativa $\mathrm{L}$.

Melandrium noctiflorum (L.) Fries

Melilotus albus Desr.

Melilotus officinalis (L.) Lam.

Mentha arvensis $\mathrm{L}$.

Menyanthes trifoliata $\mathrm{L}$.

Menziesia ferruginea $\mathrm{Sm}$.

Mertensia paniculata (Ait.) G. Don

Mimulus guttatus DC.

Minuartia biflora (L.) Sching \& Thell.

Minuartia macrocarpa (Pursh) Ostenf.

Minuartia rubella (Wahlenb.) Graebn.

Minuartia obtusiloba (Rydb.) House [=Arenaria obtusiloba (Rydb.) Fern.] ${ }^{1}$

Mitella pentandra Hook.

Moehringia lateriflora (L.) Fenzl

Moneses uniflora (L.) Gray

Myosotis alpestris F. W. Schmidt

Myrica gale $\mathrm{L}$.

Myriophyllum exalbescens Fern. [=M. spicatum L.]

Myriophyllum verticillatum $\mathrm{L}$.

Najas flexilis (Willd.) Rost. \& Schmidt

Nymphaea tetragona Georgi ${ }^{1}$

Nuphar polysepalum Engelm.

Oplopanax horridus (Smith) Miquel [=Echinopanax horridum

(Sm.) Decne. \& Planch.]

Orthilia secunda (L.) House [=Pyrola secunda L. ssp. secunda]

Osmorhiza depauperata Phill.

Oxycoccus microcarpus Turcz. ex Rupr.

Oxyria digyna (L.) Hill

Oxytropis bryophila (E. Greene) Yurtsev

Oxytropis deflexa (Pall.) DC. ${ }^{1}$

Oxytropis huddelsonii Pors.

Oxytropis maydelliana Trautv.

Oxytropus varians (Rydb.) Schumann 
Papaver alboroseum Hult.

Papaver nudicaule L.

Papaver radicatum Rottb. ssp. radicatum

Parnassia kotzebuei Cham. \& Schlecht.

Parnassia palustris L.

Parnassia palustris L. ssp. neogaea (Fern.) Hult.

Pedicularis capitata Adams.

Pedicularis labradorica Wirsing

Pedicularis lanata Cham. \& Schlecht

Pedicularis langsdorfii Fisch. ex Steven

Pedicularis parviflora J.E. Sm. ${ }^{1}$

Pedicularis verticillata $\mathrm{L}$.

Pentaphylloides floribunda (Pursh.) Loeve [=Potentilla fruticosa L.]

Petasites frigidus (L.) Franchet

Petasites sagittatus (Banks) Gray

Phalaris arundinacea $\mathrm{L}$.

Phleum commutatum Gaudin var. americanum (Fourn.) Hult.

Phleum pratense L.

Phyllodoce aleutica (Spreng.) A. A. Heller

Picea glauca (Moench) Voss

Picea mariana (Mill.) Britt., Sterns \& Pogg

Pinguicula villosa $\mathrm{L}$.

Plantago major L. var. major

Plantago maritima L. ssp. juncoides (Lam.) Hult.

Platanthera dilatata Pursh

Platanthera hyperborea (L.) Lindl. var. hyperborea

Platanthera hyperborea (L.) Lindl. var. viridiflora (Cham.) Luer

Platanthera obtusata (Pursh) Lindl.

Poa alpigena (E. Fries) Lindm.

Poa alpina $\mathrm{L}$.

Poa annua $\mathrm{L}$.

Poa arctica $\mathrm{R}$. Br.

Poa eminens Presl

Poa glauca M. Vahl.

Poa hispidula Vasey

Poa palustris L.

Poa paucispicula Scribn. \& Merr.

Poa pratensis $\mathrm{L}$.

Poa psuedoabbreviata Rosch.

Polemonium acutiflorum Willd.

Polemonium pulcherrimum Hook.

Polygonum amphibium $\mathrm{L}$.

Polygonum aviculare $\mathrm{L}$.

Polygonum convolvulus $\mathrm{L}$.

Polygonum fowleri Robins.

Polygonum lapathifolium L.

Polygonum pennsylvanicum L. ssp. oneillii (Brenckle) Hult.

Populus balsamifera L.

Populus balsamifera L. ssp. balsamifera

Populus balsamifera L. ssp. trichocarpa (Torr. \& Gray) Brayshaw

Populus tremuloides Michx.

Potamogeton alpinus Balb.

Potamogeton epihydrus Raf.

Potamogeton filiformis Pers.

Potamogeton gramineus $\mathrm{L}$.

Potamogeton natans $\mathrm{L}$.

Potamogeton pectinatus $\mathrm{L}$.

Potamogeton praelongus Wulf.

Potamogeton richardsonii (A. Bennett) Rydb. $[=P$. perfoliatus $\mathrm{L}$. ssp. richardsonii (A. Bennett) Hult.]

Potamogeton vaginatus Turcz.

Potamogeton zosterifolius Schum.

Potentilla anserina $\mathrm{L}$.

Potentilla diversifolia Lehm.

Potentilla egedii Wormsk. ssp. grandis (Torr. \& Gray) Hult.

Potentilla hyparctica Malte

Potentilla multifida $\mathrm{L}$.
Potentilla norvegica $\mathrm{L}$.

Potentilla uniflora Ledeb.

Potentilla villosa Pall.

Primula cuneifolia Ledeb. ssp. saxifragifolia (Lehm.) Smith \& Forrest

Puccinellia grandis Swallen

Puccinellia nutkaensis (Presl) Fern. \& Weath.

Puccinellia phryganodes (Trin.) Scribner \& Marr.

Pyrola asarifolia Michx.

Pyrola asarifolia Michx. var. purpurea (Bunge) Fern.

Pyrola chlorantha $\mathrm{Sw}$.

Pyrola minor L.

Ranunculus arborvitus $\mathrm{L}$.

Ranunculus cymbalaria Pursh

Ranunculus eschscholtzii Schlecht.

Ranunculus gmelini DC. ssp. gmelini

Ranunculus hyperboreus Rottb.

Ranunculus lapponicus $\mathrm{L}$.

Ranunculus macounii Britt.

Ranunculus nivalis $\mathrm{L}$

Ranunculus occidentalis Nutt.

Ranunculus pygmaeus Wahl.

Ranunculus scleratus L. ssp. multifidus (Nutt.) Hult.

Ranunculus trichophyllus Chaix

Ranunculus trichophyllus Chaix var. trichophyllus

Rhinanthus minor $\mathrm{L}$.

Rhodiola integrifolia Raf. [=Sedum rosea (L.) Scop. ssp. integrifolia (Raf.) Hult.]

Ribes hudsonianum Richards.

Ribes lacustre (Pers.) Poir. ${ }^{1}$

Ribes laxiflorum Pursh

Ribes triste Pall.

Romanzoffia sitchensis Bong.

Rorippa barbareaefolia (DC.) Kitigawa

Rorippa palustris (L.) Besser ssp. hispida (Desv.) Jonsell

Rorippa palustris (L.) Besser ssp. palustris

Rorippa sylvestris (L.) Besser

Rosa acicularis Lindl.

Rosa nutkana Presl

Rubus arcticus $\mathrm{L}$.

Rubus chamaemorus $\mathrm{L}$.

Rubus idaeus $\mathrm{L}$.

Rubus pedatus $\mathrm{Sm}$.

Rubus stellatus $\mathrm{Sm}$. [=R. arcticus L. ssp. stellatus (Sm.) Boiv. emend. Hult.]

Rumex acetosella $\mathrm{L}$.

Rumex arcticus Trautv.

Rumex crispus $\mathrm{L}$.

Rumex fenestratus Greene

Rumex transitorius $\mathrm{K}$. H. Resch

Ruppia spiralis $\mathrm{L}$.

Sagina nivalis (Lindblom) Fries

Sagina saginoides (L.) Karst.

Salicornia europaea $\mathrm{L}$.

Salix alaxensis (Anderss.) Cov.

Salix arbusculoides Anderss. ${ }^{1}$

Salix arctica Pall.

Salix barclayi Anderss.

Salix bebbiana Sarg. [=S. depressa L. ssp. rostrata (Anderss.)

Hiitonen) niphoclada]

Salix brachycarpa Nutt. ssp. niphoclada (Rydb.) Argus

Salix fuscescens Anderss.

Salix glauca L.

Salix lucida Muhl. ssp. lasiandra (Benth.) Argus [=S. lasiandra Benth.]

Salix myrtillifolia Anders. ${ }^{1}$

Salix ovalifolia Trautv.

Salix phlebophylla Anderss. ${ }^{1}$ 
Salix planifolia Pursh ssp. pulchra (Cham.) Argus [=S. pulchra Cham.]

Salix reticulata $\mathrm{L}$.

Salix rotundifolia Trautv.

Salix scouleriana Barratt

Salix sitchensis Sanson

Sambucus racemosa $\mathrm{L}$

Sanguisorba stipulata Raf.

Saxifraga adscendens $\mathrm{L}$.

Saxifraga bronchialis $\mathrm{L}$.

Saxifraga caespitosa $\mathrm{L}$.

Saxifraga calycina Sternb.

Saxifraga cernua L.

Saxifraga eschscholtzii Sternb.

Saxifraga flagellaris Willd.

Saxifraga foliolosa R. Br.

Saxifraga hirculis $\mathrm{L}$.

Saxifraga lyallii Engler ssp. hultenii (Cald. \& Sav.) Cald. \& Sav.

Saxifraga nelsoniana D. Don [=S. punctata L. ssp. pacifica Hult.]

Saxifraga nivalis $\mathrm{L}$.

Saxifraga oppositifolia L.

Saxifraga rivularis $\mathrm{L}$.

Saxifraga serpyllifolia Pursh

Saxifraga tricuspidata Rottb.

Scheuchzeria palustris L.

Schizachne purpurascens (Torr.) Swallen

Scirpus paludosus Nels.

Scirpus validus M. Vahl

Scutellaria galericulata $\mathrm{L}$.

Selaginella selaginoides (L.) Link

Senecio lugens Richardson

Senecio pauciflorus Pursh

Senecio triangularis Hook.

Senecio vulgaris $\mathrm{L}$.

Shepherdia canadensis (L.) Nutt.

Sibbaldia procumbens $\mathrm{L}$.

Silene acaulis $\mathrm{L}$.

Smilacina stellata (L.) Desf.

Solidago lepida DC.

Solidago multiradiata Ait.

Sorbus scopulina Greene

Sparganium angustifolium Michx.

Sparganium hyperboreum Laest.

Sparganium minimum (Hartm.) E. Fries

Spergula arvensis $\mathrm{L}$.

Spergularia canadensis (Pers.) G. Don

Spiraea beauverdiana Schneid.

Spiranthes romanzoffiana Cham.

Stellaria borealis Bigelow

Stellaria borealis Bigelow ssp. sitchana Steud.

Stellaria calycantha (Ledeb.) Bong.

Stellaria crassifolia Ehrh.

Stellaria humifusa Rottb.

Stellaria laeta Richards.

Stellaria longifolia Muhl. ex Willd.

Stellaria media (L.) Villars
Stellaria monantha Hult.

Stellaria umbellata Turcz.

Streptopus amplexifolius (L.) DC.

Swertia perennis L.

Swida stolonifera (Michx.) Rydb. [=Cornus stolonifera Michx.]

Taraxacum alaskanum Rydb.

Taraxacum carneocoloratum Nels.

Taraxacum officinale Weber

Thalictrum alpinum $\mathrm{L}$.

Thalictrum sparsiflorum Trucz.

Thelypteris phegopteris (L.) Solsson

Thlaspi arcticum Pors.

Tofieldia coccinea Richards.

Tofieldia glutinosa (Michx.) Pers.

Tofieldia pusilla (Michx.) Pers.

Trichophorum alpinum (L.) Pers.

Trichophorum caespitosum (L.) Hartm.

Trientalis europaea $\mathrm{L}$.

Trifolium hybridum $\mathrm{L}$.

Trifolium pratense $\mathrm{L}$.

Trifolium repens $\mathrm{L}$.

Triglochin maritimum $\mathrm{L}$.

Triglochin palustris $\mathrm{L}$.

Tripleurospermum inodoratum (L.) Schultz-Bip.

Trisetum spicatum (L.) Richter

Trisetum spicatum (L.) Richter ssp. alaskanum (Nash) Hult.

Trisetum spicatum (L.) Richter ssp. molle (Michaux) Hult.

Triticum aestivum $\mathrm{L}$.

Tsuga mertensiana (Bong.) Sarg.

Typha latifolia $\mathrm{L}$.

Urtica dioica L. ssp. gracilis (Aiton) Selander

Utricularia intermedia Hayne

Utricularia minor L.

Utricularia vulgaris L. ssp. macrorhiza (LeConte) Clauson

Vaccinium caespitosum Michx.

Vaccinium ovalifolium $\mathrm{Sm}$.

Vaccinium uliginosum $\mathrm{L}$.

Vaccinium vitis-idaea $\mathrm{L}$.

Vahlodea atropurpurea (Wahlenb.) E. Fries ssp. paramushirensis (Kudo) Hult.

Valeriana capitata Pall.

Valeriana sitchensis Bong.

Veratrum viride Ait.

Veronica americana Schwein.

Veronica wormskjoldii Roem \& Schult.

Viburnum edule (Michx.) Raf.

Vicia cracca $\mathrm{L}$.

Viola epipsila Ledeb.

Viola langsdorffii Fisch.

Viola renifolia Gray

Viola selkirkii Pursh

Woodsia ilvensis (L.) R. Br.

Zannichellia palustris $\mathrm{L}$.

Zygadenus elegans Pursh

1 Species identified during this study that were not listed in the Fort Richarson floristic inventory (Lichvar et al. 1997). Taxonomy follows Hulten (1968).

$2 \quad$ FNA $=$ The Flora of North America North of Mexico (FNAEC 1993), cited in Lichvar et al. 1997. 
Appendix E. Non-vascular plants found on Fort Richardson, south-central Alaska, 2001 (derived from Lichvar et al. 1997). Nomenclature follows that used by the University of Alaska Museum; common synonyms are listed in parentheses.

\section{Lichens}

Alectoria nigricans (Ach.) Nyl.

Alectoria ochroleuca (Hoffm.) A.Massal.

Arctoparmelia separata (Th.fr.) Hale*

Asahinea chrysantha (Tuck.) W.L.Culb. \& C.F.Culb.

(Cetraria chrysantha Tuck.)

Asahinea scholanderi (Llano) W.L.Culb. \& C.F.Culb.

Bryocaulon divergens (Ach.) Kärnefelt

(Cornicularia divergens Ach.)

Bryoria nitidula (Th.Fr.) Brodo \& D.Hawksw.

(Alectoria lanea auct.)

Candelariella terrigena Räsänen

Cetraria chlorophylla (Willd.) Vain.

Cetraria islandica (L.) Ach.

Cetraria kamczatica Savicz

Cetraria muricata (Ach.) Eckfeldt

(Coelocaulon muricatum (Ach.) J.R. Laundon

Cornicularia muricata (Ach.) Ach.)

Cetraria nigricans Nyl.

Cetrariella delisei (Bory ex Schaer.) Kärnefelt \& Thell

(Cetraria delisei (Bory ex Schaer.) Nyl.

Cetraria hiascens (Fr.) Th.Fr.)

Cladina aberrans (Abbayes) Hale \& W.L.Culb.

(Cladonia aberrans (Abbayes) Stuck.

Cladina stellaris (Opiz) Brodo var. aberrans (Abbayes) Ahti)

Cladina arbuscula (Wallr.) Hale \& W.L.Culb.

(Cladonia arbuscula (Wallr.) Flot.)

Cladina mitis (Sandst.) Hustich

(Cladonia mitis Sandst.)

Cladina rangiferina (L.) Nyl.

(Cladonia rangiferina (L.) F.H. Wigg.)

Cladina stellaris (Opiz) Brodo

(Cladonia alpestris (L.) Rabenh.

Cladonia stellaris (Opiz) Pouzar \& Vezda)

Cladina stygia (Fr.) Ahti*

Cladonia acuminata (Ach.) Norrl.

Cladonia amaurocraea (Flörke) Schaer.

Cladonia amaurocraea (Flörke) Schaer. forma celotea Ach.

Cladonia bellidiflora (Ach.) Schaer.

Cladonia borealis S.Stenroos

Cladonia cariosa (Ach.) Spreng.

Cladonia carneola (Fr.) Fr.

Cladonia cenotea (Ach.) Schaer.

Cladonia cervicornis (Ach.) Flot.

Cladonia chlorophaea (Flörke ex Sommerf.) Spreng.

(Cladonia pyxidata (L.) Hoffm. Subsp. Chlorophaea (Flörke ex Sommerf.) Spreng.)

Cladonia coccifera (L.) Willd.

(Cladonia coccifera (L.) Willd. var. coccifera)

Cladonia cornuta (L.) Hoffm.

Cladonia crispata (Ach.) Flot.

Cladonia crispata (Ach.) Flot. var. crispata

Cladonia deformis (L.) Hoffm.

Cladonia ecmocyna Leight.

Cladonia ecmocyna Leight. subsp. ecmocyna

Cladonia fimbriata (L.) Fr.

(Cladonia major (K.Hagen) Sandst.)

Cladonia gracilis (L.) Willd. subsp. gracilis

(Cladonia gracilis (L.) Willd.var. gracilis)

Cladonia gracilis (L.) Willd. subsp. turbinata

(Cladonia gracilis (L.) Willd. var. dilatata (Hoffm.) Vain.)

Cladonia gracilis (L.) Willd. subsp. vulnerata Ahti
Cladonia kanewskii Oksner

(Cladonia nipponica Asahina var. aculeata Asahina)

(Cladonia nipponica Asahina var. sachalinensis)

Cladonia ochrochlora Flörke

Cladonia phyllophora Ehrh. ex Hoffm.

(Cladonia degenerans (Flörke) Spreng.)

Cladonia pleurota (Flörke) Schaer.

(Cladonia coccifera (L.) Willd. var. pleurota (Flörke) Vain.)

Cladonia pocillum (Ach.) Grognot

Cladonia pseudostellata Asahina

Cladonia pyxidata (L.) Hoffm.

Cladonia singularis S.Hammer

Cladonia squamosa Hoffm. var. squamosa

Cladonia subulata (L.) Weber ex F.H.Wigg.

Cladonia sulphurina (Michx.) Fr.

(Cladonia deformis (L.) Hoffm. var. gonecha (Ach.) Arnold)

Cladonia thomsonii Ahti

Cladonia uncialis (L.) Weber ex F.H.Wigg.

Dactylina arctica (Richardson) Nyl.

Dactylina ramulosa (Hook.) Tuck.

Flavocetraria cucullata (Bellardi) Kärnefelt \& Thell

(Cetraria cucullata (Bellardi) Ach.)

Flavocetraria nivalis (L.) Kärnefelt \& Thell

(Cetraria nivalis (L.) Ach.)

Hypogymnia austerodes (Nyl.) Räsänen

Hypogymnia bitteri (Lynge) Ahti

Hypogymnia physodes (L.) Nyl.

Hypogymnia subobscura (Vain.) Poelt

Leprocaulon subalbicans (Lamb) Lamb \& Ward*

Lopadium pezizoideum (Ach.) Körb.

Nephroma arcticum (L.) Torss.

Nephroma bellum (Spreng.) Tuck.

Nephroma expallidum (Nyl.) Nyl.

Nephroma parile (Ach.) Ach.

Ochrolechia frigida (Sw.) Lynge

Oligotrichum hercynicum (Hedw.) Lam. \& DC.

Oligotrichum parallelum (Mitt.) Kindb.

Ophioparma lapponica (Räsänen) Hafellner \& R.W.Rogers

Pannaria pezizoides (Weber) Trevis.

Parmelia hygrophila Goward \& Ahti

Parmelia omphalodes (L.) Ach.

Parmelia saxatilis (L.) Ach.

Parmelia squarrosa Hale

Parmelia stygia (L.) Ach.

Parmelia sulcata Taylor

Parmeliopsis ambigua (Wulfen in Jacq.) Nyl.

Peltigera aphthosa (L.) Willd.

(Peltigera aphthosa (L.) Willd. var. aphthosa)

Peltigera canina (L.) Willd.

Peltigera didactyla (With.) J.R.Laundon

(Peltigera spuria (Ach.) DC.)

Peltigera horizontalis (Huds.) Baumg.

Peltigera lepidophora (Nyl. ex Vain.) Bitter

Peltigera leucophlebia (Nyl.) Gyeln.

(Peltigera aphthosa (L.) Willd. var. leucophlebia Nyl.)

Peltigera malacea (Ach.) Funck

Peltigera membranacea (Ach.) Nyl.

Peltigera neopolydactyla (Gyelnik) Gyelnik*

(Peltigera occidentalis (E. Dahl) Kristinsson)

Peltigera praetextata (Flörke ex Sommerf.) Zopf

Peltigera rufescens (Weiss) Humb.

(Peltigera canina (L.) Willd. var. refescens (Weiss) Mudd)

Peltigera scabrosa Th.Fr 
Pertusaria panyrga (Ach.) A. Massal.*

Physcia dubia (Hoffm.) Lettau

Platismatia glauca (L.)W.L.Culb. \& C.F.Culb.

Pseudephebe pubescens (L.) M.Choisy

Pseudocyphellaria crocata(L.) Vain.

Psoroma hypnorum (Vahl) Gray

Ramalina thrausta (Ach.) Nyl.

Rhizocarpon geographicum (L.) DC.

Solorina crocea (L.) Ach.

Sphaerophorus fragilis (L.) Pers.

Sphaerophorus globosus (Huds.) Vain.

(Sphaerophorus coralloides Pers.)

Stereocaulon alpinum Laurer ex Funck

Stereocaulon arenarium (Savicz) I.M.Lamb

Stereocaulon condensatum Hoffm.

Stereocaulon glareosum (Savicz) H.Magn.

Stereocaulon glareosum (Savicz) H.Magn. var. brachyphylloides I.M.Lamb

Stereocaulon glareosum (Savicz) H.Magn. var. glareosum

Stereocaulon grande (H.Magn.) H.Magn.

Stereocaulon groenlandicum (Å.E.Dahl) I.M.Lamb

Stereocaulon paschale (L.) Hoffm.

Stereocaulon rivulorum H.Magn.

Stereocaulon tomentosum $\mathrm{Fr}$.

Thamnolia subuliformis (Ehrh.) W.L.Culb.

Thamnolia vermicularis (Sw.) Ach. ex Schaer.

Tuckermannopsis inermis (Nyl.) Kärnefelt*

Umbilicaria proboscidea (L.) Schrad.

Umbilicaria rigida (Du Rietz) Frey

Umbilicaria torrefacta (Lightf.) Schrad.

Vulpicida pinastri (Scop.) Mattson \& M.J.Lai

Vulpicida tilesii (Ach.) Mattson \& M.J.Lai

(Cetraria tilesii Ach.)

Xanthoria candelaria (L.) Th.Fr.

Hepatics

Aneura pinguis (L.) Dumort.

Barbilophozia kunzeana (Huebener) Gams

(Orhocaulis kunzeanus (Huebener) H.Buch)

Barbilophozia lycopodioides (Wallr.) Loeske

Barbilophozia quadriloba (Lindb.) Loeske

Blasia pusilla $\mathrm{L}$

Blepharostoma trichophyllum (L.) Dumort.

Cephalozia ambigua C.Massal.

Cephaloziella arctica Bryhn \& Douin (s.1.)*

Cephalozia bicuspidata (L.) Dumort.

(Cephalozia lammersiana (Huebener) Carring.)

Gymnocolea acutiloba (Schiffn.) Müll.Frib.

(Gymnocolea inflata (Huds.) Dumort. var. acutiloba (Kaal.) S.W.Arnell)

Gymnomitrion obtusum (Lindb.) Pearson

Jungermannia subelliptica (Lindb. ex Kaal.) Levier

Lobaria linita (Ach.) Rabenh.

Lobaria pulmonaria (L.) Hoffm.

Lobaria scrobiculata (Scop.) DC. in Lam. \& DC.

Lophozia longidens (Lindb.) Macoun

Lophozia ventricosa (Dicks.) Dumort.

Marchantia polymorpha L. subsp. montivagans Bischl. \&

Boisselier

Marchantia polymorpha L. subsp. ruderalis Bischl. \& Boisselier

Pellia neesiana (Gottsche) Limpr.

Pleurocladula albescens (Hook.) Grolle

(Pleuroclada albescens (Hook.) Spruce)

Ptilidium californicum (Austin) Underw.

Ptilidium ciliare (L.) Hampe

Ptilidium pulcherrimum (Weber) Hampe

Ptilium crista-castrensis (Hedw.) De Not.

Scapania scandica (Arnell \& H.Buch) Macvicar

Mosses

Abietinella abietina (Hedw.) M.Fleisch.

(Thuidium abietinum (Hedw.) Schimp. in Bruch, Schimp. \& W. Gümbel)
Amblystegium serpens (Hedw.) Schimp. in B.S.G*

Andreaea blyttii Schimp.

Andreaea nivalis Hook.

Andreaea rupestris Hedw.

Andreaea rupestris Hedw. var. rupestris

Aulacomnium androgynum (Hedw.) Schimp.

Aulacomnium palustre (Hedw.) Schwägr.

Aulacomnium turgidum (Wahlenb.) Schwägr *

Bartramia ithyphylla Brid.

Brachythecium coruscum Hag.*

Brachythecium reflexum (Starke in Web \& Mohr) Schimp. In B.S.G.*

Brachythecium salebrosum (Web. \& Mohr) Schimp. in B.S.G.*

Bryoerythrophyllum recurvirostrum (Hedw.) P.C.Chen var. recurvirostrum

Bryum caespiticium Hedw.

Bryum pseudotriquetrum (Hedw.) P.Gaertn., B.Mey. \& Scherb.

(Bryum neodamense Itzigs.)

(Bryum ovatum Jur.)

Buxbaumia aphylla Hedw.

Calliergon cordifolium (Hedw.) Kindb.

Calliergon giganteum (Schimp.) Kindb.*

Calliergon richardsonii (Mitt.) Kindb.

Calliergon stramineum (Brid.) Kindb.

Campylium hispidulum (Brid.) Mitt.*

Ceratodon purpureus (Hedw.) Brid.

Climacium dendroides (Hedw.) F.Weber \& D.Mohr

Conostomum tetragonum (Hedw.) Lindb.

Cratoneuron filicinum (Hedw.) Spruce

Dicranella schreberiana (Hedw.) Hilf. ex H.A.Crum \& L.E.Anderson

Dicranoweisia crispula (Hedw.) Lindb. ex Milde

Dicranum bonjeanii De Not.*

Dicranum brevifolium (Lindb.) Lindb.

Dicranum elongatum Schleich. ex Schwägr.

Dicranum flexicaule Brid.*

(Dicranum congestum Brid*)

Dicranum majus $\mathrm{Sm}$.

Dicranum polysetum $\mathrm{Sw}$.

Dicranum scoparium Hedw.

Distichium capillaceum (Hedw.) Bruch \& Schimp.

Ditrichum flexicaule (Schwägr.) Hampe

Drepanocladus aduncus (Hedw.) Warnst.

Encalypta brevicolla (Bruch \& Schimp. in Bruch, Schimp. \& W.Gümbel) Bruch ex Angstr. var. brevicolla

Encalypta brevicoll (Bruch \& Schimp. in Bruch, Schimp. \& W.Gümbel) Bruch ex Angstr. subsp. brevicolla

Encalypta brevipes Schljakov

Encalypta procera Bruch

Encalypta rhaptocarpa Schwägr.

(Encalypta vulgaris Hedw. var. rhabdocarpa (Schwagr.) E.Lawton)

Eurhynchium pulchellum (Hedw.) Jenn.

Helodium blandowii (Web. \& Mohr) Warnst.*

Hylocomiastrum pyrenaicum (Spruce) M.Fleisch.

(Hylocomiastrum pyrenaicum (Spruce) Lindb.)

Hylocomium splendens (Hedw.) Schimp. in Bruch, Schimp. \& W.Gümbel

(Hylocomium alaskanum (Lesq. \& James) Austin

Hylocomium splendens (Hedw.) Schimp. in Bruch, Schimp. \& W.Gümbel var. alaskanum

Hylocomium splendens (Hedw.) Schimp. in Bruch, Schimp. \& W.Gümbel var. obtusifolium)

Hylocomium splendens (Hedw.) Schimp. in Bruch, Schimp. \& W.Gümbel

Hypnum plicatulum (Lindb.) Jaeg.*

Hypnum revolutum (Mitt.) Lindb.

Hypnum subimponens Lesq.*

Kiaeria blyttii (Schimp.) Broth.

(Arctoa blyttii (Schimp.) Loeske)

Kiaeria glacialis (Berggr.) I.Hagen 
Kiaeria starkei (F.Weber \& D.Mohr) I.Hagen

Leptobryum pyriforme (Hedw.) Wilson

Loeskypnum badium (Hartm.) H.K.G.Paul

(Drepanocladus badius (Hartm.) G. Roth)

Oligotrichum hercynicum (Hedw.) Lam. \& DC.

Oligotrichum parallelum (Mitt.) Kindb.

Oncophorus wahlenbergii Brid.*

Oncophorus virens (Hedw.) Brid.

Orthotrichum obtusifolium Brid.

Paludella squarrosa (Hedw.) Brid.

Philonotis fontana (Hedw.) Brid.

(Philonotis tomentella Molendo )

Plagiomnium ellipticum (Brid.) T. Kop.

(Plagiomnium rugicum (Laur.) T. Kop.)

Plagiomnium medium (Bruch \& Schimp. in Bruch, Schimp. \& W.Gümbel) T.Kop.

(Mnium medium Bruch \& Schimp. in Bruch, Schimp. \& W.Gümbel)

Plagiothecium piliferum (Sw. ex Hartm.) Schimp. in B.S.G.*

Pleurozium schreberi (Brid.) Mitt.

Pogonatum dentatum (Brid.) Brid.

(Pogonatum capillare (Michx.) Brid.)

Pogonatum urnigerum (Hedw.) P.Beauv.

Pohlia cruda (Hedw.) Lindb.

Pohlia crudoides (Sull. \& Lesq.) Broth.

Pohlia drummondii (Müll.Hal.) A.L.Andrews

Pohlia filum (Schimp.) Mårtensson

(Pohlia gracilis (Bruch \& Schimp. in Bruch, Schimp. \& W.Gümbel) Lindb.

Pohlia rothii (Correns in Limpr. ) Broth.

Pohlia schleicheri H.A. Crum)

Pohlia ludwigii (Spreng. ex Schwägr.) Broth.

Pohlia nutans (Hedw.) Lindb.

(Pohlia schimperi (Mull. Hal.) Lindb.)

Pohlia proligera (Lindb. ex Breidl.) Lindb. ex Arnell

Pohlia wahlenbergii (F.Weber \& D.Mohr) A.L.Andrews

(Mniobryum albicans L(Wahlenb.) Limpr.

Mniobryum wahlenbergii (F. Weber and D. Mohr) Jenn.

Pohlia albicans Lindb.)

Polytrichastrum alpinum (Hedw.) G.L.Sm.

(Pogonatum alpinum (Hedw.) Röhl.)

Polytrichastrum sexangulare (Brid.) G.L.Sm. var. sexangulare

(Polytrichastrum sexangulare Brid.)

Polytrichum commune Hedw.

Polytrichum commune Hedw. var. commune

(Polytrichum commune Hedw. var. perigoniale (Michx.) Hampe)

Polytrichum commune Hedw. var. jensenii (Hag.) M'"nk. in Warnst*

(Polytrichum jensenii (Hag.)*)

Polytrichum hyperboreum R.Br.

Polytrichum juniperinum Hedw.

Polytrichum longisetum Brid.*

(Polytrichum gracile Bryhn*)

Polytrichum piliferum Hedw.

Polytrichum strictum Brid.

(Polytrichum affine Funck

Polytrichum juniperinum Hedw. var. gracilius Wahlenb.)

Polytrichum swartzii Hartm.

(Polytrichum algidum I. Hagan \& C.E.O. Jensen)

Pseudobryum cinclidioides (Huebener) T.Kop.

(Mnium cinclidioides Huebener)

Pylaisiella polyantha (Hedw.) Grout
Racomitrium affine (Schleich. ex F.Weber \& D.Mohr) Lindb.

Racomitrium canescens (Hedw.) Brid.

Racomitrium ericoides (F.Weber ex Brid.) Brid.

(Racomitrium canescens (Hedw.) Brid.var.ericoides (Brid.) Schimp. \& W.Gümbel

Racomitrium canescens (Hedw.) Brid. var. strictum Schlieph. in Limpr.)

Racomitrium fasciculare (Hedw.) Brid.

Racomitrium heterostichum (Hedw.) Brid.*

Racomitrium lanuginosum (Hedw.) Brid.

Rhizomnium andrewsianum (Steere) T.Kop.

Rhizomnium gracile T.Kop.

Rhizomnium magnifolium (Horik.) T.Kop.

(Mnium punctatum Hedw. var. elatum Schimp.

Thizomnium perssonii T.Kop.)

Rhizomnium nudum (E.Britton \& R.S.Williams) T.Kop.

(Mnium nudum (Britt \& Williams) T Kop.)

Rhizomnium pseudopunctatum (Bruch \& Schimp.) T.Kop.

(Mnium pseudopunctatum Bruch \& Schimp.)

Rhytidiadelphus triquetrus (Hedw.) Warnst.

Rhytidium rugosum (Hedw.) Kindb.

Sanionia uncinata (Hedw.) Loeske

(Drepanocladus uncinatus (Hedw.) Warnst.)

Schistostega pennata (Hedw.) F.Weber \& D.Mohr

Scorpidium scorpioides (Hedw.)Limpr.*

Sphagnum angustifolium (C.E.O.Jensen ex Russow) C.E.O.Jensen in Tolf

(Sphagnum recurvum P.Beauv. var. tenue H.Klinggr.)

Sphagnum aongstroemii C.Hartm.

Sphagnum balticum (Russ.) Russ. C.Jens.*

Sphagnum capillifolium (Ehrh.) Hedw.

(Sphagnum capillaceum (Weiss) Schrank

Sphagnum nemoreum Scop. auct. plur.)

Sphagnum centrale C.E.O.Jensen in Arnell \& C.E.O.Jensen

Sphagnum fallax (Klinggr.) Klinggr.*

Sphagnum fuscum (Schimp.) H.Klinggr.

Sphagnum girgensohnii Russow

Sphagnum lenense H.Lindb. in Pohle

Sphagnum magellanicum Brid.

Sphagnum majus (Russ.) C.Jens.*

Sphagnum papillosum Lindb.

Sphagnum recurvum P.Beauv.

(Sphagnum recurvum P.Beauv. var. recurvum)

Sphagnum riparium Ångstr.

Sphagnum rubellum Wils.*

Sphagnum russowii Warnst.

(Sphagnum robustum (Warnst.) Röll)

Sphagnum squarrosum Crome

Sphagnum subsecundum Nees in Sturm var. subsecundum

Sphagnum teres (Schimp.) Ångstr.

Sphagnum warnstorfii Russ.*

Tetralophozia setiformis (Ehrh.) Schljakov*

Tetraphis pellucida Hedw.

Timmia austriaca Hedw.

Tomentypnum nitens (Hedw.) Loeske

Tortella fragilis (Drumm.) Limpr.

Warnstorfia exannulata (Schimp. in Bruch, Schimp. \& W.Gümbel) Loeske

(Drepanocladus exannulatus (Schimp. In Bruch, Schimp. \& W. Gümbel) Warnst.)

Warnstorfia fluitans (Hedw.) Loeske*

Warnstorfia trichophylla (Warnst.) Tuom. \& T.Kop.

(Drepanocladus trichophyllus (Warnst.) Podp.)

* Species identified during this study that were not listed in the Fort Richardson floristic inventory (Lichvar et al. 1997). Nomenclature follows that of the National Plants Database (USDA). 


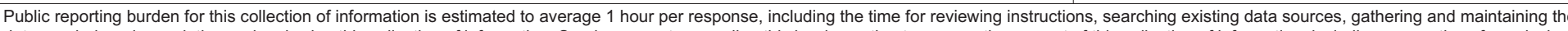

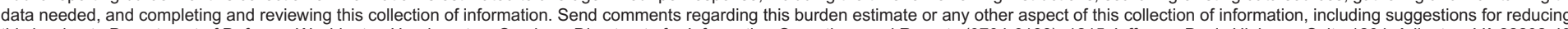

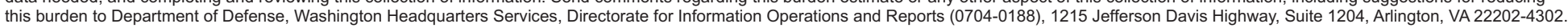

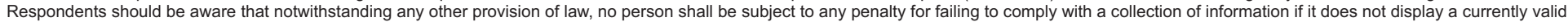
OMB control number. PLEASE DO NOT RETURN YOUR FORM TO THE ABOVE ADDRESS.
1. REPORT DATE (DD-MM-YY)
2. REPORT TYPE
September 2003

4. TITLE AND SUBTITLE

An Ecological Land Survey for

Fort Richardson, Alaska
3. DATES COVERED (From - To)

\section{5a. CONTRACT NUMBER}

5b. GRANT NUMBER

5c. PROGRAM ELEMENT NUMBER

5d. PROJECT NUMBER

5e. TASK NUMBER

5f. WORK UNIT NUMBER

8. PERFORMING ORGANIZATION REPORT

ERDC/CRREL TR-03-19

10. SPONSOR / MONITOR'S ACRONYM(S)

11. SPONSOR / MONITOR'S REPORT NUMBER(S)
U.S. Army Alaska, Directorate of Public Works

600 Richardson Avenue, Fort Richardson

Anchorage, Alaska 99505

\section{DISTRIBUTION / AVAILABILITY STATEMENT}

Approved for public release; distribution is unlimited.

Available from NTIS, Springfield, Virginia 22161.

\section{SUPPLEMENTARY NOTES}

\section{ABSTRACT}

An ecological land survey (ELS) of Fort Richardson land was conducted to map ecosystems at three spatial scales to aid in the management of natural resources. In an ELS, an attempt is made to view landscapes not just as aggregations of separate biological and earth resources, but as ecological systems with functionally related parts that can provide a consistent conceptual framework for ecological applications. Field surveys at 132 plots along 16 toposequences and at 99 other plots were used to identify relationships among physiography, geomorphology, soils, hydrology, and vegetation. The relationships revealed that the various ecosystem components were closely related to fire effects and geomorphic processes, such as floodplain development, landslide and slope instability, and coastal flooding. Associations among vegetation structures and geomorphic units were used to identify 51 ecotypes (local-scale ecosystems) that were effective at differentiating dominant species and plant associations. Ecosystem maps were developed at three spatial scales. Forty-six ecotypes (1:20,000 scale), derived from the integrated terrain unit (ITU) mapping, differentiated areas with homogeneous topography, terrain, soil, surface form, hydrology, and vegetation. Vegetation (structure and composition) and environmental (elevation, organic matter accumulation, depth to rock, water depths, $\mathrm{pH}$, and electrical conductivity) characteristics of ecotypes were summarized using data obtained from field surveys. Sixteen ecosections (1:100,000 scale) were aggregated from the ecotypes to differentiate areas that are homogeneous with respect to geomorphic features and soil texture, and thus have recurring patterns of soils and vegetation at various successional stages. Four ecodistricts and eight ecosubdistricts (1:250,000) were developed from separate mapping of Landsat imagery to differentiate broader areas with similar physiography, geology, and geomorphology. This hierarchical linkage of ecological characteristics within a spatial database facilitates the evaluation of land capabilities and sensitivities and provides flexibility for addressing a wide range of land management objectives.

\begin{tabular}{|lll|}
\hline 15. SUBJECT TERMS & Alaska & Geomorphology \\
& Classification & Mapping \\
Ecosystem & Vegetation \\
\hline
\end{tabular}

\begin{tabular}{|c|c|c|c|c|c|}
\hline \multicolumn{3}{|c|}{ 16. SECURITY CLASSIFICATION OF: } & \multirow{2}{*}{$\begin{array}{l}\text { 17. LIMITATION OF } \\
\text { OF ABSTRACT }\end{array}$} & \multirow{2}{*}{$\begin{array}{l}\text { 18. NUMBER } \\
\text { OF PAGES }\end{array}$} & \multirow{2}{*}{\begin{tabular}{|l|} 
19a. NAME OF RESPONSIBLE PERSON \\
19b. TELEPHONE NUMBER (include area code)
\end{tabular}} \\
\hline a. REPORT & b. ABSTRACT & c. THIS PAGE & & & \\
\hline $\mathrm{U}$ & $\mathrm{U}$ & $\mathrm{U}$ & $\mathrm{U}$ & 108 & \\
\hline
\end{tabular}

